\title{
The 1997 reference of diffuse night sky brightness ${ }^{\star}$
}

Ch. Leinert ${ }^{1}$, S. Bowyer ${ }^{2}$, L.K. Haikala ${ }^{3}$, M.S. Hanner ${ }^{4}$, M.G. Hauser ${ }^{5}$, A.-Ch. Levasseur-Regourd ${ }^{6}$, I. Mann ${ }^{7}$, K. Mattila ${ }^{3}$, W.T. Reach ${ }^{8}$, W. Schlosser ${ }^{9}$, H.J. Staude ${ }^{1}$, G.N. Toller ${ }^{10}$, J.L. Weiland ${ }^{11}$, J.L. Weinberg ${ }^{12}$, and A.N. Witt ${ }^{13}$

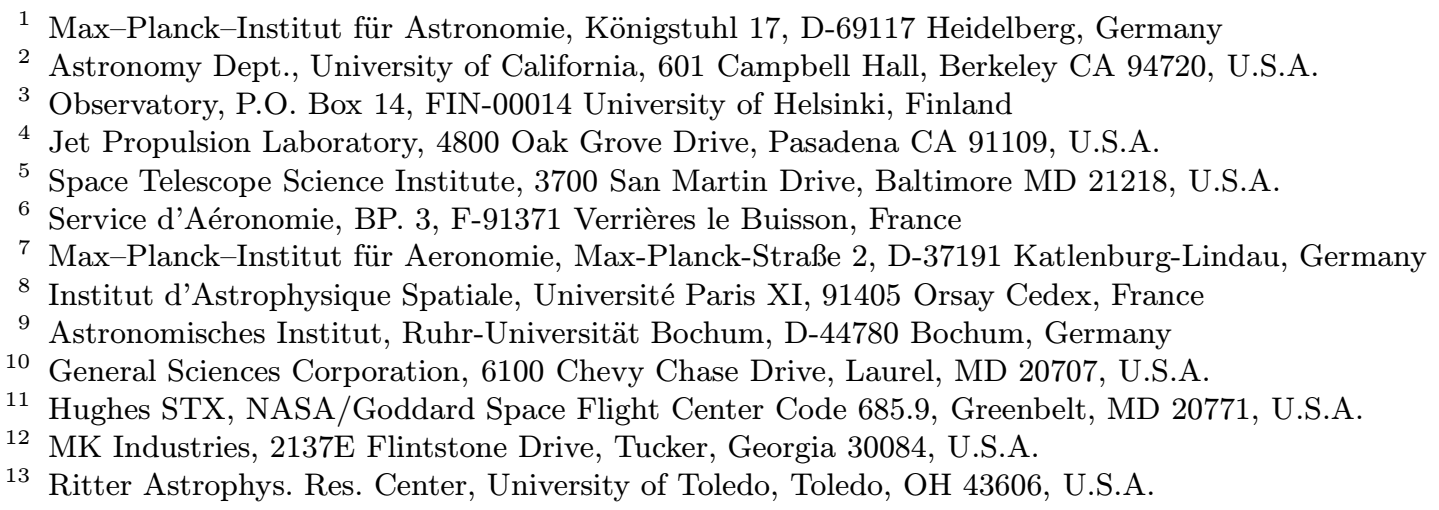

Received August 7; accepted September 8, 1997

\begin{abstract}
In the following we present material in tabular and graphical form, with the aim to allow the nonspecialist to obtain a realistic estimate of the diffuse night sky brightness over a wide range of wavelengths from the far UV longward of Ly $\alpha$ to the far-infrared. At the same time the data are to provide a reference for cases in which background brightness has to be discussed, including the planning for space observations and the issue of protection of observatory sites. We try to give a critical presentation of the status at the beginning of 1997 .
\end{abstract}

Key words: diffuse radiation - interplanetary medium - atmospheric effects - astronomical dabases: miscellaneous - infrared: general — ultraviolet: general

\section{Contents}

1. Overview

2. Brightness units

3. Coordinate transformations

4. Total sky brightness

5. Tropospheric scattering

6. Airglow

6.1 Airglow spectrum, UV to IR

6.2 Dependence on zenith distance

6.3 Variations

Send offprint requests to: Ch. Leinert

* Prepared by members of Commission 21 "Light of the night sky" of the IAU, including most of the recent (vice-)presidents.
6.4 Geocorona

6.5 Interplanetary emissions

6.6 Shuttle glow

7. Light pollution

8. Zodiacal light

8.1 Overview and general remarks

8.2 Heliocentric dependence

8.3 Zodiacal light at $1 \mathrm{AU}$ in the visual

8.4 Wavelength dependence and colour

8.5 Zodiacal light in the infrared

8.6 Zodiacal light in the ultraviolet

8.7 Seasonal variations

8.8 Structures in the zodiacal light

8.9 The zodiacal light seen from other places

9. Coronal brightness and polarisation

10. Integrated starlight

10.1 Model predictions based on star counts

10.2 Ultraviolet

10.3 Ground-based UBVR photometry

10.4 Pioneer 10/11 spaceborne visual photometry

10.5 Near-and mid-infrared

11. Diffuse galactic light

11.1 Overview

11.2 Visual

11.3 Near-infrared

11.4 Thermal infrared

11.5 Ultraviolet

12. Extragalactic background light 


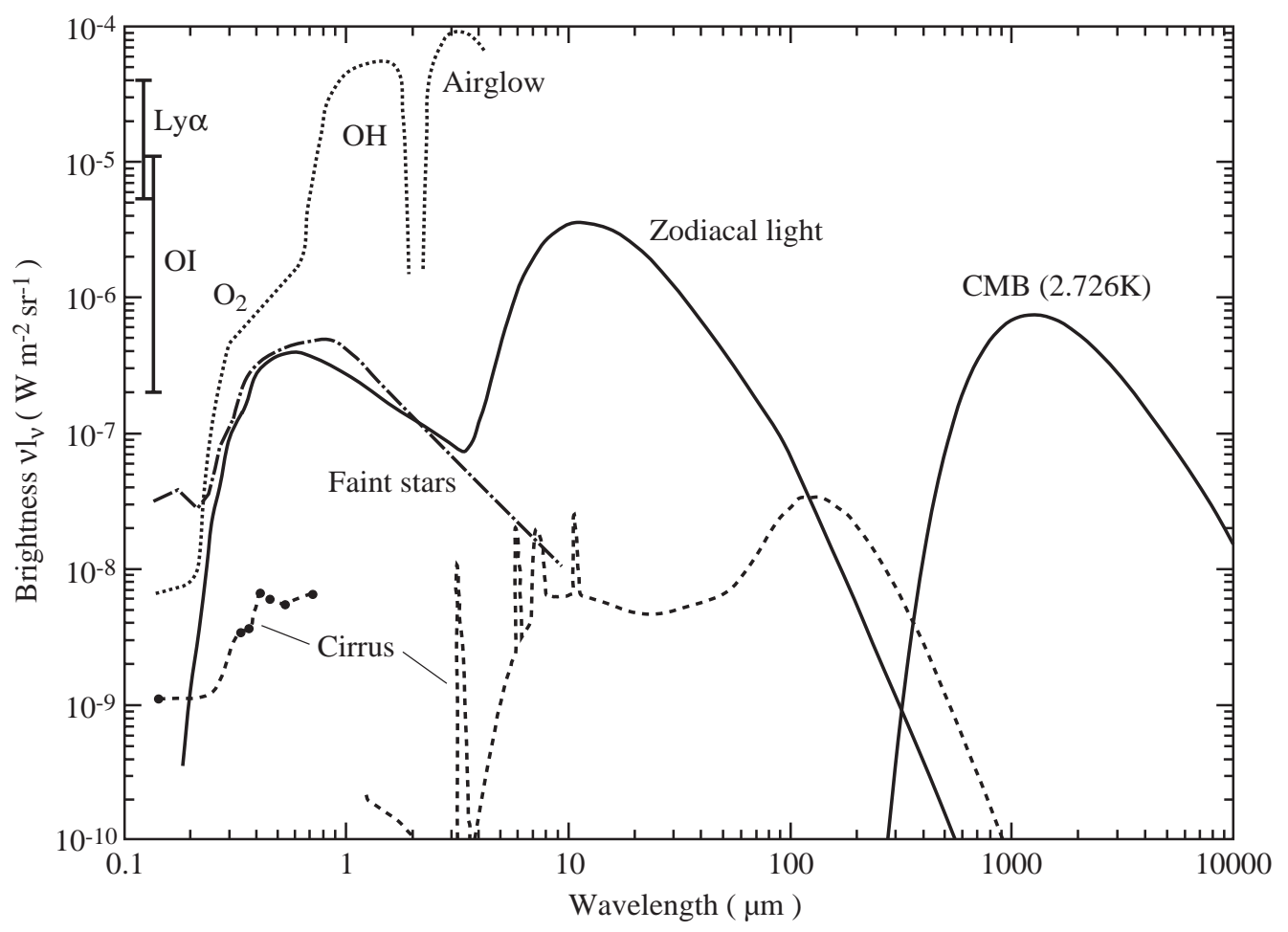

Fig. 1. Overview on the brightness of the sky outside the lower terrestrial atmosphere and at high ecliptic and galactic latitudes. The zodiacal emission and scattering as well as the integrated light of stars are given for the South Ecliptic Pole $\left(l=276^{\circ}\right.$, $\left.b=-30^{\circ}\right)$. The bright magnitude cut-off for the stellar component is $V=6.0 \mathrm{mag}$ for $0.3-1 \mu \mathrm{m}$. In the infrared, stars brighter than $15 \mathrm{Jy}$ between 1.25 and $4.85 \mu \mathrm{m}$ and brighter than $85 \mathrm{Jy}$ at $12 \mu \mathrm{m}$ are excluded. No cut-off was applied to the UV data, $\lambda \leq 0.3 \mu \mathrm{m}$. The interstellar cirrus component is normalized for a column density of $10^{20} \mathrm{H}$-atoms $\mathrm{cm}^{-2} \mathrm{corresponding} \mathrm{to} \mathrm{a}^{2}$ visual extinction of $0.053 \mathrm{mag}$. This is close to the values at the darkest patches in the sky. Source for the long-wavelength data, $\lambda \geq 1.25 \mu \mathrm{m}$, are COBE DIRBE and FIRAS measurements as presented by Désert et al. (1996). The IR cirrus spectrum is according to the model of Désert et al. (1990) fitted to IRAS photometry. The short-wavelength data, $\lambda \leq 1.0 \mu \mathrm{m}$, are from the following sources: zodiacal light: Leinert \& Grün (1990); integrated starlight: $\lambda \leq 0.3 \mu \mathrm{m}$, Gondhalekar $(1990), \lambda \geq 0.3 \mu \mathrm{m}$, Mattila (1980); cirrus: $\lambda=0.15 \mu \mathrm{m}$, Haikala et al. (1995), $\lambda=0.35-0.75 \mu \mathrm{m}$, Mattila \& Schnur (1990), Mattila (1979). The geocoronal Lyman $\alpha(121.6 \mathrm{~nm})$ and the OI $(130.4,135.6 \mathrm{~nm})$ line intensities were as measured with the Faint Object Camera of the Hubble Space Telescope at a height of $610 \mathrm{~km}$ (Caulet et al. 1994). The various references for the airglow emission can be found in Sect. 6

\section{Overview}

This paper is concerned with the night sky brightness from the far UV $(\approx 100 \mathrm{~nm})$ to the far infrared $(\approx 200 \mu \mathrm{m})$.

Quite a few sources contribute to the diffuse brightness of the moonless sky ( $\left.I_{\text {night sky }}\right)$ in this wavelength range: - airglow from the upper atmosphere $\left(I_{\mathrm{A}}\right)$.

-Zodiacal light, both as scattered sunlight and thermal emission of interplanetary dust particles, from interplanetary space $\left(I_{\mathrm{ZL}}\right)$. (In the far UV interplanetary Ly $\alpha$ emission is important.)

- Integrated starlight $\left(I_{\mathrm{ISL}}\right)$ of the stars not individually accounted for

-diffuse galactic light $\left(I_{\mathrm{DGL}}\right)$, in the UV and visual mainly reflections off interstellar dust particles. Their infrared thermal emission is known as "cirrus" since the pioneering IRAS observations. It dominates the sky brightness in the far-infrared. Interstellar gas contributes line emissions over all of our wavelength range.

- Extragalactic background light $\left(I_{\mathrm{EBL}}\right)$ in addition to the radiation of individually detected galaxies.

The combined light of these radiations is attenuated by atmospheric extinction, while tropospheric scattering of the infalling flux adds a non-negligible brightness component $\left(I_{\mathrm{sca}}\right)$.

Formally, the above statements may be expressed as

$I_{\text {night sky }}=\left(I_{\mathrm{A}}+I_{\mathrm{ZL}}+I_{\mathrm{ISL}}+I_{\mathrm{DGL}}+I_{\mathrm{EBL}}\right) \cdot \mathrm{e}^{-\tau}+I_{\mathrm{sca}}$.

It should be noted that the "extinction coefficient" $\tau$ (which depends on wavelength $\lambda$, zenith distance $z$, height of the observer and change of the atmospheric conditions with time) for diffuse sources has a value different from that determined for stars. The scattered light $I_{\text {sca }}$ not only contains additional contributions due to stars and galaxies otherwise accounted for individually, but, 
Table 1. Conversion factors for ultraviolet brightness units

\begin{tabular}{c|c|c|c|c|c}
$\begin{array}{c}\text { Wavelength } \\
(\mathrm{nm})\end{array}$ & $I_{\lambda}\left[\mathrm{W} / \mathrm{m}^{2} \mathrm{sr} \mu \mathrm{m}\right]$ & $I_{\lambda}\left[\mathrm{erg} / \mathrm{cm}^{2} \mathrm{~s} \mathrm{sr} \AA\right]$ & $I_{\nu}[\mathrm{Jy} / \mathrm{sr}]$ & $\mathrm{R} / \AA$ & $\begin{array}{c}\text { Conversion factor } \\
I_{\nu} \rightarrow I_{\lambda}\end{array}$ \\
\hline 30 & $6.6210^{-10}$ & $6.6210^{-11}$ & 0.199 & $1.2610^{-5}$ & $3.33110^{-9}$ \\
60 & $3.3110^{-10}$ & $3.3110^{-11}$ & 0.398 & $1.2610^{-5}$ & $8.32810^{-10}$ \\
100 & $1.9910^{-10}$ & $1.9910^{-11}$ & 0.663 & $1.2610^{-5}$ & $2.99810^{-10}$ \\
$121.6^{a}$ & $1.6310^{-10}$ & $1.6310^{-11}$ & 0.856 & $1.2610^{-5}$ & $2.02710^{-10}$ \\
150 & $1.3210^{-10}$ & $1.3210^{-11}$ & 0.994 & $1.2610^{-5}$ & $1.33210^{-10}$ \\
200 & $9.9310^{-11}$ & $9.9310^{-12}$ & 1.325 & $1.2610^{-5}$ & $7.49510^{-11}$ \\
250 & $7.9510^{-11}$ & $7.9510^{-12}$ & 1.657 & $1.2610^{-5}$ & $4.79710^{-11}$ \\
300 & $6.6210^{-11}$ & $6.6210^{-12}$ & 1.988 & $1.2610^{-5}$ & $3.33110^{-11}$ \\
350 & $5.6810^{-11}$ & $5.6810^{-12}$ & 2.319 & $1.2610^{-5}$ & $2.44710^{-11}$ \\
400 & $4.9710^{-11}$ & $4.9710^{-12}$ & 2.650 & $1.2610^{-5}$ & $1.87410^{-11}$ \\
500 & $3.9710^{-11}$ & $3.9710^{-12}$ & 2.120 & $1.2610^{-5}$ & $1.19910^{-11}$ \\
$656.3^{b}$ & $3.0310^{-11}$ & $3.0310^{-12}$ & 4.349 & $1.2610^{-5}$ & $6.96010^{-12}$ \\
$1 \mu \mathrm{m}$ & $1.9910^{-11}$ & $1.9910^{-12}$ & 6.628 & $1.2610^{-5}$ & $2.99810^{-12}$ \\
$2 \mu \mathrm{m}$ & $9.9310^{-12}$ & $9.9310^{-13}$ & 13.25 & $1.2610^{-5}$ & $7.49510^{-13}$ \\
$4 \mu \mathrm{m}$ & $4.9710^{-12}$ & $4.9710^{-13}$ & 26.50 & $1.2610^{-5}$ & $1.87410^{-13}$ \\
\hline
\end{tabular}

${ }^{a} \operatorname{Ly} \alpha, \quad{ }^{b} \mathrm{H} \alpha$.

Table 2. Conversion factors for visual brightness units

\begin{tabular}{|c|c|c|c|c|c|c|c|c|}
\hline \multirow{2}{*}{$\begin{array}{l}\text { Wavelength } \\
\qquad(\mu \mathrm{m})\end{array}$} & \multicolumn{2}{|c|}{$1 \mathrm{MJy} / \mathrm{sr}$ corresponds to } & \multirow{2}{*}{$\begin{array}{c}F_{\nu}[\mathrm{Jy}]^{a} \\
\text { for } 0 \mathrm{mag}\end{array}$} & \multicolumn{2}{|c|}{$1 S_{10}$ unit $^{b}$ corresponds to } & \multicolumn{3}{|c|}{$1 S 1 \odot_{\odot}$ unit $^{c}$ corresponds to } \\
\hline & $I_{\lambda}\left[\mathrm{W} / \mathrm{m}^{2} \mathrm{sr} \mu \mathrm{m}\right]$ & $\mathrm{R} / \AA$ & & $I_{\lambda}\left[\mathrm{W} / \mathrm{m}^{2} \mathrm{sr} \mu \mathrm{m}\right]$ & $I_{\nu}[\mathrm{Jy} / \mathrm{sr}]$ & $I_{\lambda}\left[\mathrm{W} / \mathrm{m}^{2} \mathrm{sr} \mu \mathrm{m}\right]$ & $S_{10}$ units & $I_{\nu}[\mathrm{Jy} / \mathrm{sr}]$ \\
\hline $0.36(U)$ & $2.3110^{-5}$ & 5.27 & 1810 & $1.3710^{-8}$ & 590 & $6.7010^{-9}$ & 0.488 & 290 \\
\hline $0.44(B)$ & $1.5510^{-5}$ & 4.31 & 4260 & $2.1710^{-8}$ & 1400 & $1.1910^{-8}$ & 0.550 & 770 \\
\hline $0.502 \pm 12 \mathrm{~nm}$ & $1.1910^{-5}$ & 3.78 & 3960 & $1.5510^{-8}$ & 1300 & $1.2810^{-8}$ & 0.825 & 1070 \\
\hline $0.530 \pm 3.5 \mathrm{~nm}$ & $1.0710^{-5}$ & 3.58 & 3790 & $1.3310^{-8}$ & 1240 & $1.2410^{-8}$ & 0.935 & 1160 \\
\hline $0.55(V)$ & $9.9110^{-6}$ & 3.45 & 3640 & $1.1810^{-8}$ & 1200 & $1.1810^{-8}$ & 1.0 & 1200 \\
\hline $0.64\left(R_{\mathrm{C}}\right)$ & $7.3210^{-6}$ & 2.96 & 3080 & $7.4010^{-9}$ & 1010 & $1.0510^{-8}$ & 1.42 & 1440 \\
\hline $0.70\left(R_{\mathrm{J}}\right)$ & $6.1210^{-6}$ & 2.71 & 2840 & $5.7010^{-9}$ & 930 & $9.2110^{-9}$ & 1.61 & 1510 \\
\hline $0.79\left(I_{\mathrm{C}}\right)$ & $4.8010^{-6}$ & 2.34 & 2550 & $4.0210^{-9}$ & 840 & $7.8010^{-9}$ & 1.94 & 1620 \\
\hline $0.90\left(I_{\mathrm{J}}\right)$ & $3.7010^{-6}$ & 2.11 & 2250 & $2.7310^{-9}$ & 740 & $5.7610^{-9}$ & 2.11 & 1560 \\
\hline $1.25(J)$ & $1.9210^{-6}$ & 1.52 & 1570 & $9.8910^{-10}$ & 515 & $2.9310^{-9}$ & 2.97 & 1530 \\
\hline $1.65(H)$ & $1.1010^{-6}$ & 1.15 & 1020 & $3.6910^{-10}$ & 335 & $1.4110^{-9}$ & 3.84 & 1290 \\
\hline $2.2(K)$ & $6.1910^{-7}$ & 0.862 & 636 & $1.2910^{-10}$ & 210 & $5.2410^{-10}$ & 4.06 & 850 \\
\hline
\end{tabular}

${ }^{a}$ References: for $U, B, V, R_{\mathrm{C}}, I_{\mathrm{C}}$ Bessell (1979); for $R_{\mathrm{J}}, I_{\mathrm{J}}$ Allen (1985); for $J, H, K$ Bessell \& Brett (1988); for $502 \mathrm{~nm}$ and $530 \mathrm{~nm}$ Hayes (1985). The references give $F_{\nu}$ or $F_{\lambda}$ for a star of magnitude zero, with uncertainties of about $2 \%-5 \%$. They are transformed to $S_{10}$ units by: 1 zeroth magnitude star $/ \mathrm{sr}=3.046 S_{10}$ units.

${ }^{b}$ By definition $1 S_{10}$ unit corresponds to $27.78 \mathrm{mag} / \square^{\prime \prime}$, while $22 \mathrm{mag} / \square^{\prime \prime}=205 S_{10}$.

${ }^{c}$ The definition of this unit depends on the solar $U B V R I J H K$ values, which are uncertain by several \% beyond $1.0 \mu \mathrm{m}$ and below $400 \mathrm{~nm}$. References: for $U, B, V, R_{\mathrm{J}}, I_{\mathrm{J}}$ Allen (1985); for $R_{\mathrm{C}}, I_{\mathrm{C}}$ Bessell \& Brett (1988), Taylor (1992); for $J, H, K$ Alonso et al. (1995); for 502 $\mathrm{nm}$ and $530 \mathrm{~nm}$ Neckel \& Labs (1984).

increasingly more important, the light pollution due to the ever-growing man-made lighting.

For space observations atmospheric extinction and scattering are irrelevant, but other complexities like instrumental stray light of lunar, terrestrial or solar radiation may arise. For low orbits, spacecraft-induced glow phenomena may be present.

Quite understandably then, extracting accurate brightness values from Eq. (1) is a difficult task, and the past has seen a measure of disagreement between individual determinations. In the following we want to summarise what consensus has been obtained in this field during the last years, in order to provide a basis for easier reference and comparability.

The aim of this article is to provide the reader with comparatively easy access to agreed-upon or at least recommended values of night sky brightness. Inevitably this requires smoothing and interpolating of data. Therefore we want to give at the same time sufficient information on original publications to give an impression on the grade of agreement or disagreement of the available data and to allow the reader who wants to do so to draw his own conclusions. 
Table 3. Conversion factors for infrared brightness units

\begin{tabular}{|c|c|c|c|c|c|c|c|}
\hline \multirow{2}{*}{$\begin{array}{l}\text { Wavelength } \\
\qquad(\mu \mathrm{m})\end{array}$} & \multicolumn{2}{|c|}{$1 \mathrm{MJy} / \mathrm{sr}$ corresponds to } & \multicolumn{2}{|c|}{$F_{\nu}[\mathrm{Jy}]$ for star } & \multicolumn{3}{|c|}{$1 S_{10}$ unit corresponds to } \\
\hline & $I_{\lambda}\left[\mathrm{W} / \mathrm{m}^{2} \mathrm{sr} \mu \mathrm{m}\right]$ & $I_{\lambda}\left[\operatorname{cgs}^{a}\right]$ & of $0 \mathrm{mag}$ & Ref. & $I_{\lambda}\left[\mathrm{W} / \mathrm{m}^{2} \mathrm{sr} \mu \mathrm{m}\right]$ & $I_{\lambda}\left[\operatorname{cgs}^{a}\right]$ & $I_{\nu}[\mathrm{Jy} / \mathrm{sr}]$ \\
\hline $1.25(J)$ & $1.9210^{-6}$ & $1.9210^{-7}$ & 1570 & 1 & $9.8910^{-10}$ & $9.8910^{-11}$ & 515 \\
\hline $1.65(H)$ & $1.1010^{-6}$ & $1.1010^{-7}$ & 1020 & 1 & $3.6910^{-10}$ & $3.6910^{-11}$ & 335 \\
\hline $2.2(K)$ & $6.1910^{-7}$ & $6.1910^{-8}$ & 636 & 1 & $1.2910^{-10}$ & $1.2910^{-11}$ & 209 \\
\hline $3.5(L)$ & $2.4510^{-7}$ & $2.4510^{-8}$ & 281 & 1 & $2.2610^{-11}$ & $2.2610^{-12}$ & 92.3 \\
\hline $3.8\left(L^{\prime}\right)$ & $2.0810^{-7}$ & $2.0810^{-8}$ & 235 & 1 & $1.6010^{-11}$ & $1.6010^{-12}$ & 77.2 \\
\hline $4.8(M)$ & $1.3010^{-7}$ & $1.3010^{-8}$ & 152 & 1 & $6.5010^{-12}$ & $6.5010^{-13}$ & 49.9 \\
\hline 8.4 & $4.2510^{-8}$ & $4.2510^{-9}$ & 58 & 2 & $8.0910^{-13}$ & $8.0910^{-14}$ & 19.0 \\
\hline 10 & $3.0010^{-8}$ & $3.0010^{-9}$ & 40 & 3 & $3.9410^{-13}$ & $3.9410^{-14}$ & 13.1 \\
\hline $10.6(N)$ & $2.6710^{-8}$ & $2.6710^{-9}$ & 36 & 3 & $3.1510^{-13}$ & $3.1510^{-14}$ & 11.8 \\
\hline 12 & $2.0810^{-8}$ & $2.0810^{-9}$ & 28 & 4 & $1.9110^{-13}$ & $1.9110^{-14}$ & 9.19 \\
\hline 20 & $7.5010^{-9}$ & $7.5010^{-10}$ & 10.4 & 3 & $2.5610^{-14}$ & $2.5610^{-15}$ & 3.41 \\
\hline $21(Q)$ & $6.8010^{-9}$ & $6.8010^{-10}$ & 9.4 & 3 & $2.1010^{-14}$ & $2.1010^{-15}$ & 3.09 \\
\hline 25 & $4.8010^{-9}$ & $4.8010^{-10}$ & 6.7 & 4 & $1.0610^{-14}$ & $1.0610^{-15}$ & 2.20 \\
\hline 60 & $8.3310^{-10}$ & $8.3310^{-11}$ & 1.19 & 4 & & & \\
\hline 90 & $3.7010^{-10}$ & $3.7010^{-11}$ & & & & & \\
\hline 100 & $3.0010^{-10}$ & $3.0010^{-11}$ & & & & & \\
\hline 135 & $1.6410^{-10}$ & $1.6410^{-11}$ & & & & & \\
\hline 175 & $9.7911^{-10}$ & $9.7910^{-12}$ & & & & & \\
\hline 200 & $7.5010^{-11}$ & $7.5010^{-12}$ & & & & & \\
\hline 240 & $5.2110^{-11}$ & $5.2110^{-12}$ & & & & & \\
\hline
\end{tabular}

${ }^{a}$ unit is $\left[\mathrm{erg} / \mathrm{cm}^{2} \mathrm{~s}\right.$ sr $\left.\AA\right]$.

${ }^{1}$ Bessell \& Brett $(1988){ }^{2}$ Gillett \& Stein $(1971){ }^{3}$ Rieke et al. (1985) ${ }^{4}$ Neugebauer et al. (1988). The above references give $F_{\nu}$ or $F_{\lambda}$ for a star of magnitude zero, with uncertainties of about $2 \%-5 \%$. These values are transformed to $S_{10}$ units by: 1 zeroth magnitude $\mathrm{star} / \mathrm{sr}=3.046 S_{10}$ units.

We will go through the components basically in the order in which they appear in Eq. (1), and for each component try to provide information on the visual, infrared and ultraviolet wavelength ranges.

\section{Brightness units}

There are a number of different brightness units in use in the different fields of night sky brightness with their individual traditions and advantages. Rather than trying the Sisiphus work of standardising the use of brightness units, we give here conversion tables. These should help to transform whatever was given in an original reference to the desired physical units and allow intercomparison between different sources. As a rule, we will in the quantitative information on night sky brightness stay with the units of the original papers. The units come in two groups:

(1) physical units:

- photons $/ \mathrm{cm}^{2}$ s sr $\AA$.

- Rayleigh $/ \AA[R / \AA]$. Originally a measure of the emission in a column through the atmosphere, it also may be understood as a sky brightness of $10^{6} / 4 \pi$ photons $/ \mathrm{cm}^{2}$ s sr $\AA$.

$-F_{\lambda}$ in $\mathrm{W} / \mathrm{m}^{2}$ sr $\mu$ as well as in $\mathrm{W} / \mathrm{cm}^{2}$ sr $\mu$ and - in the cgs system - in $\mathrm{erg} / \mathrm{cm}^{2}$ s sr $\AA$, where

$1 \mathrm{~W} / \mathrm{m}^{2}$ sr $\mu=10^{-4} \mathrm{~W} / \mathrm{cm}^{2}$ sr $\mu=0.10 \mathrm{erg} / \mathrm{cm}^{2} \mathrm{~s}$ sr $\AA$.

- $F_{\nu}$ in $\mathrm{MJy} / \mathrm{sr}$ or $\mathrm{Jy} / \mathrm{sr}$, where $1 \mathrm{Jy}=10^{-26}$
$\mathrm{W} \mathrm{m}^{-2} \mathrm{~Hz}^{-1}$.

Note that $\nu F_{\nu}\left[\mathrm{W} / \mathrm{m}^{2} \mathrm{sr} \mathrm{Hz}\right]=\lambda F_{\lambda}\left[\mathrm{W} / \mathrm{m}^{2} \mathrm{sr} \mu \mathrm{m}\right]$ and $F_{\lambda}\left[\mathrm{W} / \mathrm{m}^{2} \mathrm{sr} \mu \mathrm{m}\right]=c / \lambda^{2}[\mathrm{~Hz} / \mathrm{m}] \cdot 10^{-6} \cdot F_{\nu}\left[\mathrm{W} / \mathrm{m}^{2} \mathrm{sr} \mathrm{Hz}\right]$.

(2) Traditional units:

- $S_{10}$ units [tenth magnitude star per degree squared]. This is the brightness equivalent to the flux of a star of magnitude 10 (tenth magnitude in the wavelength range under consideration) distributed over one degree squared. Basically it refers to A0 stars, which essentially have the same magnitude in all wavelength bands. The $S_{10}$ unit was convenient in terms of calibration by stars and in that by its use most values of the night sky brightness in the visual fall in the range $100-1000$.

$-B / \overline{B_{\odot}}$ (units of the mean brightness of the solar disk, mainly used in observations of the solar corona).

- $S 10_{\odot}$ [solar type stars of tenth magnitude per degree squared]. The unit has also been called $S 10$ or $S_{10}$ (vis). This unit is a convenient measure of the zodiacal light in the visual, where its spectral energy distribution would be equal to the solar one for neutral scattering. With $V_{\odot}=-26.74$ and the mean solid angle of the Sun of $6.8010^{-5}$ sr (Allen 1985), we have, denoting the solar irradiance at $1 \mathrm{AU}$ as $F_{\odot}$, $1 S 10_{\odot}=6.6110^{-12} F_{\odot} / \mathrm{sr}=4.5010^{-16} \mathrm{~B} / \overline{B_{\odot}}$.

As representation of the solar radiation we use the solar spectral irradiance data of Neckel \& Labs (1984). This understanding of the $S 10_{\odot}$ unit almost 
exactly agrees with the definition given by Sparrow \& Weinberg (1976).

Because of the different traditions we give the conversion tables separately for the ultraviolet, the visual and the infrared. Note that the conversion factors to physical units may be slightly different for a narrow-band filter and a broad-band filter at the same wavelength. A useful quantitity to remember when working with the conversion tables is the energy of a $1 \mu \mathrm{m}$ photon: $h \nu=1.98610^{-19}$ Ws.

\section{Coordinate transformations}

Object coordinates are usually given in the equatorial $\alpha$, $\delta$ sytem.

The zodiacal light is given in terms of ecliptic coordinates $\lambda-\lambda_{\odot}, \beta$ with the zero point of $\lambda$ in the Sun. Description of a line of sight by elongation $\epsilon$ and inclination $i$ also is common. For the relation between these two sets of coordinates see Fig. 2 and Sect. 3.5 below.

Integrated starlight is naturally presented in galactic coordinates $l, b$.

For estimates of the diffuse background brightness at a given position, transformation between these three systems is necessary. Figures 3-7 provide a simple way to do so graphically with about one-degree accuracy, which is sufficient for many applications. The underlying transformation equations are summarised below for ease of access.

Airglow, extinction and scattering are best described in the local horizontal system $A, z$ (azimuth, zenith distance). The transformation to the other systems depends on time and on the observer's geographic coordinates. For the horizontal system, only the equations for the transformation to the equatorial system are given.

\subsection{Ecliptic and equatorial coordinates}

The obliquity of the ecliptic is essentially constant, $\epsilon=$ $23.446^{\circ}$ for equinox 1950 , respectively $\epsilon=23.439^{\circ}$ for equinox 2000.

The precession of the vernal equinox along the ecliptic is $p_{0}=50.3^{\prime \prime} /$ year. Hence

$\lambda_{2000}=\lambda_{1950}+0.698^{\circ}$.

The north ecliptic pole is at $\alpha=18 \mathrm{~h}, \delta=90^{\circ}-\epsilon$.

The north celestial pole is at $\lambda=90^{\circ}, \beta=90^{\circ}-\epsilon$.

Both $\alpha$ and $\lambda$ are counted eastward from the vernal equinox. Apart from the minimal change in $\epsilon$, the transformation equations then are the same for 1950 and 2000:

\subsubsection{Transformation $\alpha, \delta \rightarrow \lambda, \beta$}

$$
\begin{aligned}
\sin \beta & =\sin \delta \cos \epsilon-\cos \delta \sin \epsilon \sin \alpha \\
\cos \lambda & =\cos \alpha \cos \delta / \cos \beta \\
\sin \lambda & =[\sin \delta \sin \epsilon+\cos \delta \cos \epsilon \sin \alpha] / \cos \beta
\end{aligned}
$$

3.1.2. Transformation $\lambda, \beta \rightarrow \alpha, \delta$

$$
\begin{aligned}
\sin \delta & =\sin \beta \cos \epsilon+\cos \beta \sin \epsilon \sin \lambda \\
\cos \alpha & =\cos \lambda \cos \beta / \cos \delta \\
\sin \alpha & =[-\sin \beta \sin \epsilon+\cos \beta \cos \epsilon \sin \lambda] / \cos \delta .
\end{aligned}
$$

\subsection{Galactic and equatorial coordinates}

By IAU decision, for equinox 1950 the north galactic pole (NGP) is at $\alpha=12 \mathrm{~h} 49.0 \mathrm{~m}, \delta=27.4^{\circ}$ and the celestial pole at $l=123.0^{\circ}, b=27^{\circ} 24.0^{\prime}$. Hence the ascending node of the galactic equator is at $\alpha_{0}=18 \mathrm{~h} 49.0 \mathrm{~m}=282.25^{\circ}$, $l_{0}=33.0^{\circ}$. For equinox 2000, the coordinates of the north galactic pole are $\alpha=12 \mathrm{~h} 51.42 \mathrm{~m}, \delta=27^{\circ} 07.8^{\prime}$, and we have $\alpha_{0}=282.86^{\circ}, l_{0}=32.93^{\circ}$. The inclination of the galactic equator with respect to the ecliptic is $90^{\circ}-\delta_{\mathrm{NGP}}$. As $\alpha$ and $\lambda, l$ is also counted eastwards.

With these parameters, the transformations are as follows:

\subsubsection{Transformation $\alpha, \delta \rightarrow l, b$}

$$
\begin{aligned}
\sin b=\sin \delta \quad \sin \delta_{\mathrm{NGP}}-\cos \delta \cos \delta_{\mathrm{NGP}} \sin \left(\alpha-\alpha_{0}\right) \\
\cos \left(l-l_{0}\right)=\cos \left(\alpha-\alpha_{0}\right) \cos \delta / \cos b \\
\sin \left(l-l_{0}\right)=\left[\sin \delta \cos \delta_{\mathrm{NGP}}+\right. \\
\\
\left.\cos \delta \sin \delta_{\mathrm{NGP}} \sin \left(\alpha-\alpha_{0}\right)\right] / \cos b .
\end{aligned}
$$

\subsubsection{Transformation $l, b \rightarrow \alpha, \delta$}

$$
\begin{aligned}
& \sin \delta=\sin b \sin \delta_{\mathrm{NGP}}+\cos b \cos \delta_{\mathrm{NGP}} \sin \left(l-l_{0}\right) \\
& \cos \left(\alpha-\alpha_{0}\right)= \cos \left(l-l_{0}\right) \cos b / \cos \delta \\
& \sin \left(\alpha-\alpha_{0}\right)= {\left[-\sin b \cos \delta_{\mathrm{NGP}}+\right.} \\
&\left.\cos b \sin \delta_{\mathrm{NGP}} \sin \left(l-l_{0}\right)\right] / \cos \delta
\end{aligned}
$$

\subsection{Galactic and ecliptic coordinates}

In ecliptic coordinates, for equinox 1950 the north galactic pole is at $\lambda=179.32^{\circ}, \beta=29.81^{\circ}$, and the ascending node of the galactic equator at $\lambda_{0}=269.32^{\circ}$, $l_{1}=6.38^{\circ}$. For equinox 2000 , the coordinates of the north galactic pole are $\lambda=180.02^{\circ}, \beta=29.81^{\circ}$, and we have $\lambda_{0}=270.02^{\circ}, l_{1}=6.38^{\circ}$. The inclination of the galactic equator with respect to the ecliptic is $90^{\circ}-\beta_{\mathrm{NGP}}$. As already mentioned, $l$ is counted eastwards. With these parameters, the transformations are as follows: 
3.3.1. Transformation $\lambda, \beta \rightarrow l, b$

$$
\begin{aligned}
\sin b=\sin \beta & \sin \beta_{\mathrm{NGP}}-\cos \beta \cos \beta_{\mathrm{NGP}} \sin \left(\lambda-\lambda_{0}\right) \\
\cos \left(l-l_{1}\right)= & \cos \left(\lambda-\lambda_{0}\right) \cos \beta / \cos b \\
\sin \left(l-l_{1}\right)= & {\left[\sin \beta \cos \beta_{\mathrm{NGP}}+\right.} \\
& \left.\cos \beta \sin \beta_{\mathrm{NGP}} \sin \left(\lambda-\lambda_{0}\right)\right] / \cos b .
\end{aligned}
$$

3.3.2. Transformation $l, b \rightarrow \lambda, \beta$

$$
\begin{aligned}
& \sin \beta=\sin b \sin \beta_{\mathrm{NGP}}+\cos b \cos \beta_{\mathrm{NGP}} \sin \left(l-l_{1}\right) \\
& \cos \left(\lambda-\lambda_{0}\right)= \cos \left(l-l_{1}\right) \cos b / \cos \beta \\
& \sin \left(\lambda-\lambda_{0}\right)= {\left[-\sin b \cos \beta_{\mathrm{NGP}}+\right.} \\
&\left.\cos b \sin \beta_{\mathrm{NGP}} \sin \left(l-l_{1}\right)\right] / \cos \beta .
\end{aligned}
$$

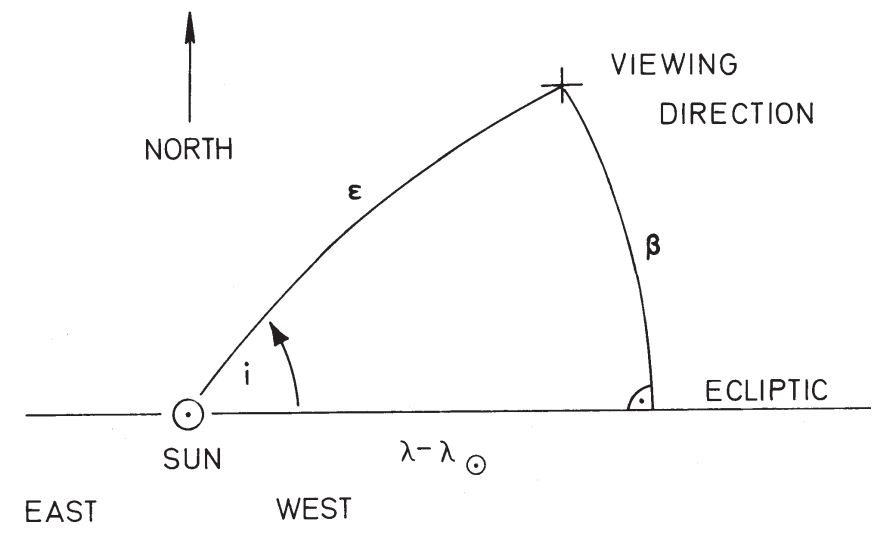

\subsection{Altazimuth and equatorial coordinates}

The transformation depends on local sidereal time $\Theta$ and on geographical latitude $\phi$. Instead of elevation, zenith distance $z=$ will be used. The zenith distance of the celestial pole is $90^{\circ}-\phi$. Both, azimuth $A$ and hour angle $t=\Theta-\alpha$ are counted from the meridian through west.

3.4.1. Transformation $\alpha, \delta \rightarrow A, z$

$$
\begin{aligned}
\cos z & =\sin \delta \sin \phi+\cos \delta \cos \phi \cos (\Theta-\alpha) \\
\cos A & =[-\sin \delta \cos \phi+\cos \delta \sin \phi \cos (\Theta-\alpha)] / \sin z(9) \\
\sin A & =\sin (\Theta-\alpha) \cos \delta / \sin z
\end{aligned}
$$

\subsubsection{Transformation $A, z \rightarrow \alpha, \delta$}

$$
\begin{aligned}
\sin \delta & =\cos z \sin \phi-\sin z \cos \phi \cos A \\
\cos (\Theta-\alpha) & =[\cos z \cos \phi+\sin z \sin \phi \cos A] / \cos \delta(10) \\
\sin (\Theta-\alpha) & =\sin A \sin z / \cos \delta .
\end{aligned}
$$

\subsection{Alternate ecliptic coordinates}

Instead of $\lambda-\lambda_{\odot}, \beta$ also a sun-centered polar coordinate system is used. Its coordinates are the angular distance from the sun, called elongation $\epsilon$, and a position angle $i$, counted from the ecliptic counterclockwise,called inclination. The relation between the two sets of coordinates, when describing the position of a field-of-view with respect to the sun, is shown in Fig. 2.
3.5.1. Transformation $\lambda-\lambda_{\odot}, \beta \rightarrow \epsilon, i$

$$
\begin{aligned}
\cos \epsilon & =\cos \left(\lambda-\lambda_{\odot}\right) \cos \beta \\
\cos i & =\cos \beta \sin \left(\lambda-\lambda_{\odot}\right) / \sin \epsilon \\
\sin i & =\sin \beta / \sin \epsilon .
\end{aligned}
$$

$$
\begin{aligned}
& \text { 3.5.2. Transformation } \epsilon, i \rightarrow \lambda-\lambda_{\odot}, \beta \\
& \begin{aligned}
\sin \beta & =\sin i \sin \epsilon \\
\cos \left(\lambda-\lambda_{\odot}\right) & =\cos \epsilon / \cos \beta \\
\sin \left(\lambda-\lambda_{\odot}\right) & =\cos i \sin \epsilon / \cos \beta
\end{aligned}
\end{aligned}
$$

The reader is cautioned that in some papers the differential helioecliptic longitude $\lambda-\lambda_{\odot}$ may be called "elongation" or may be designated as " $\epsilon$ ", contrary to our definition of elongation $\epsilon$ as the angular distance from the sun to the field-of-view. 

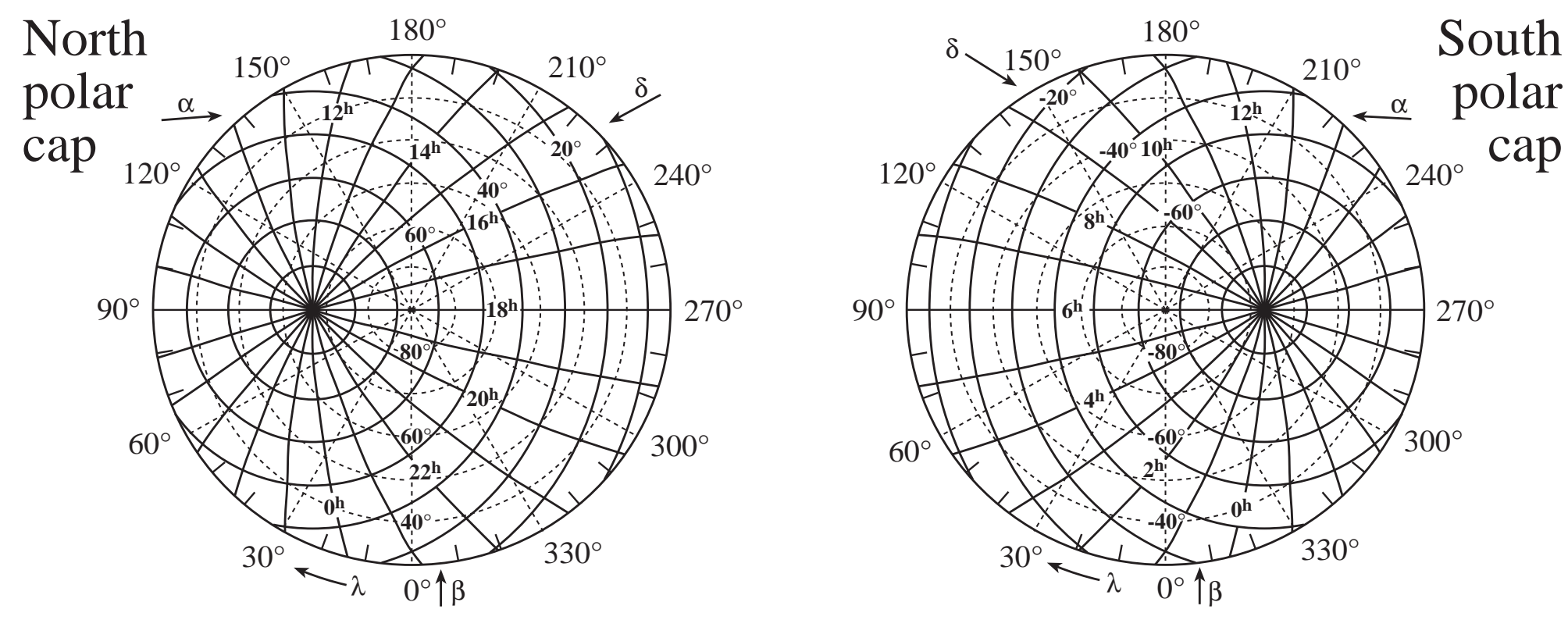

South

cap

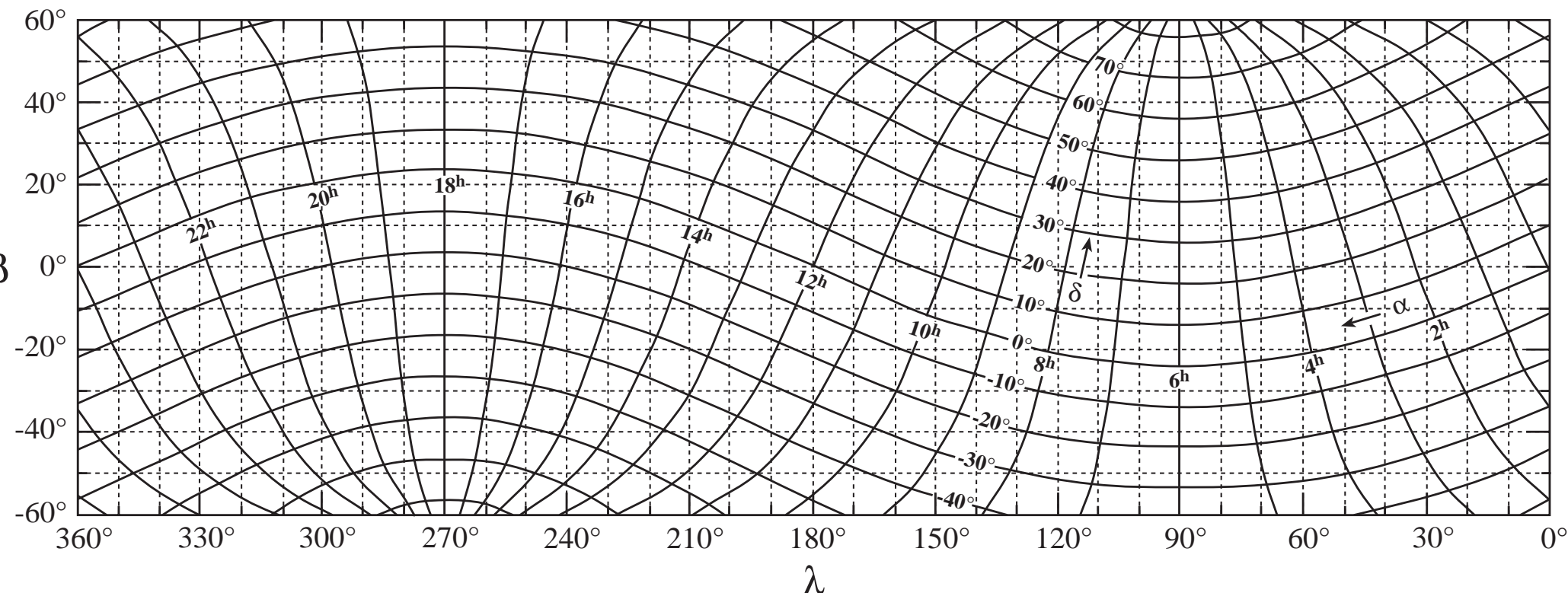


North
polar

cap

$90^{\circ}$

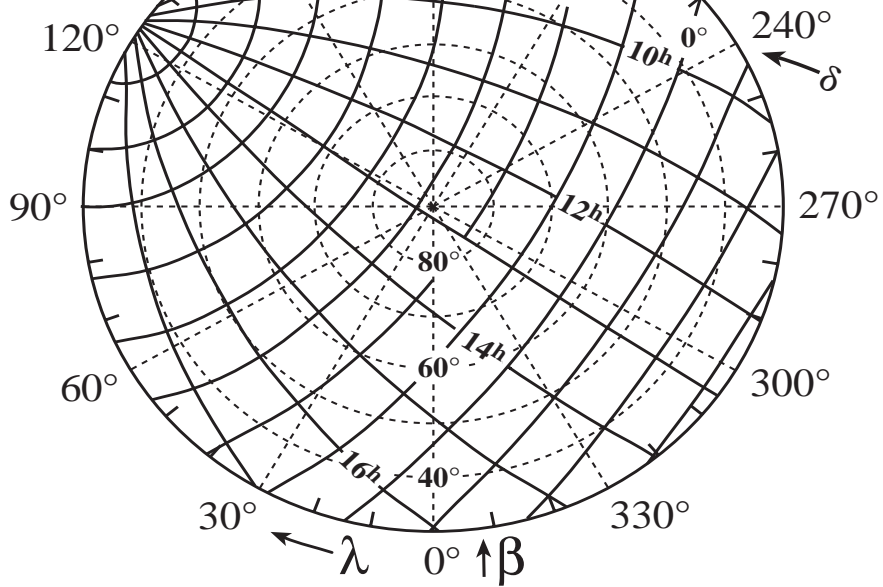

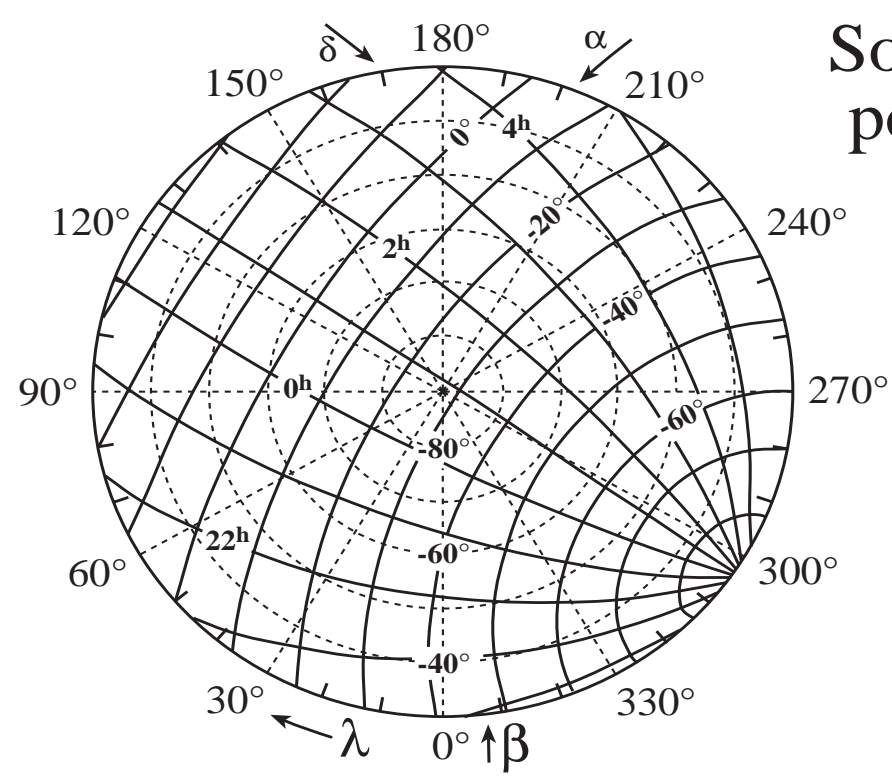

South

polar

cap

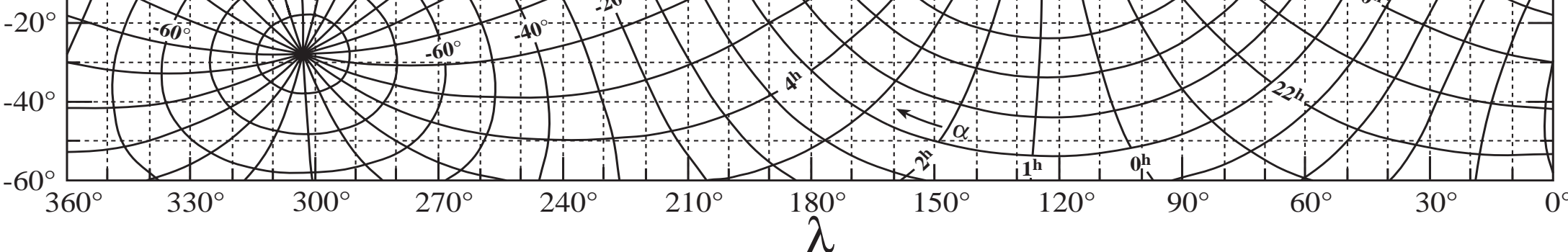




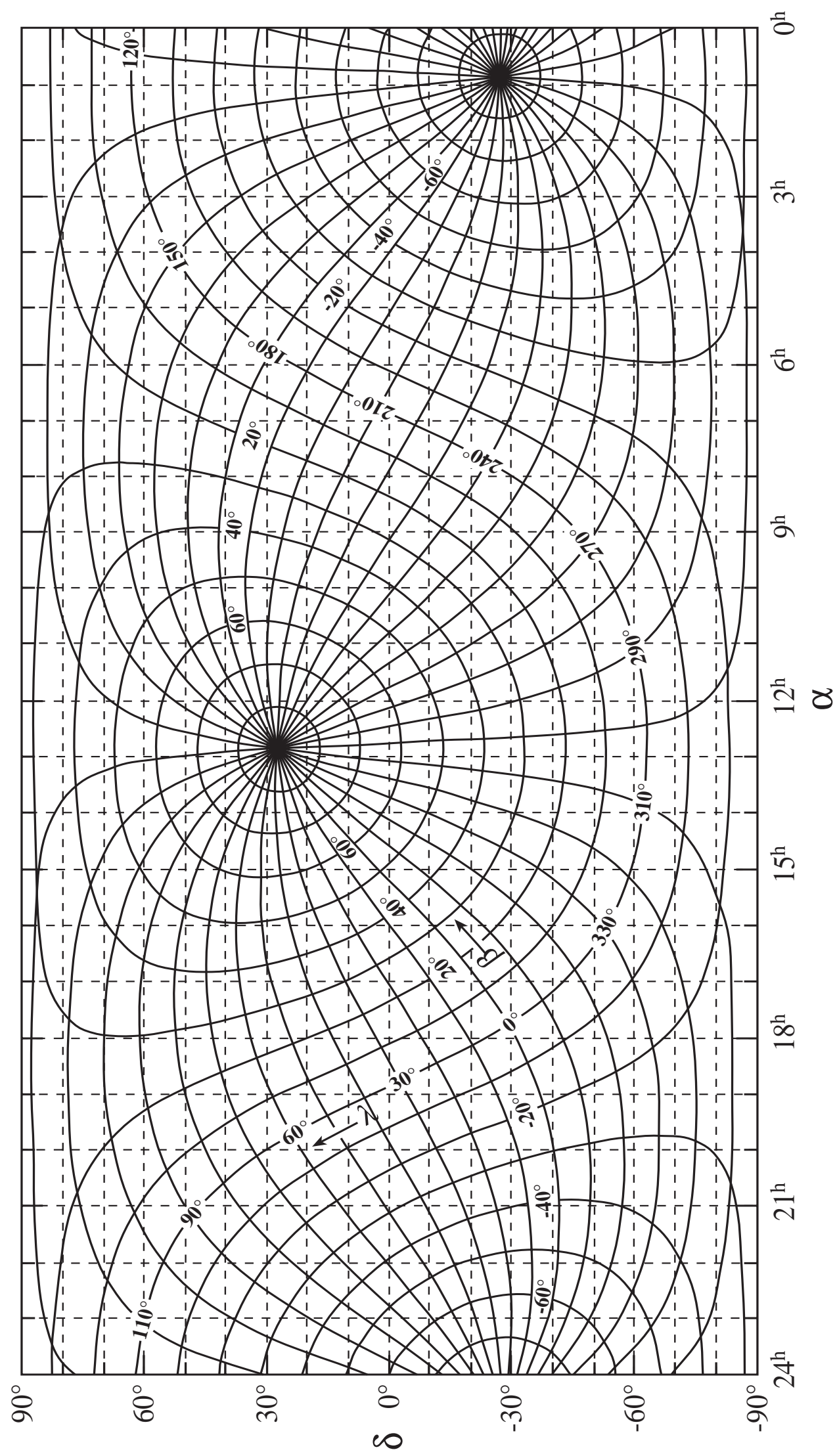

Fig. 5. Relation between coordinates $l, b$ (lines) and $\alpha, \delta$ (underlying dotted grid) for equinox 2000 - alternative projection 

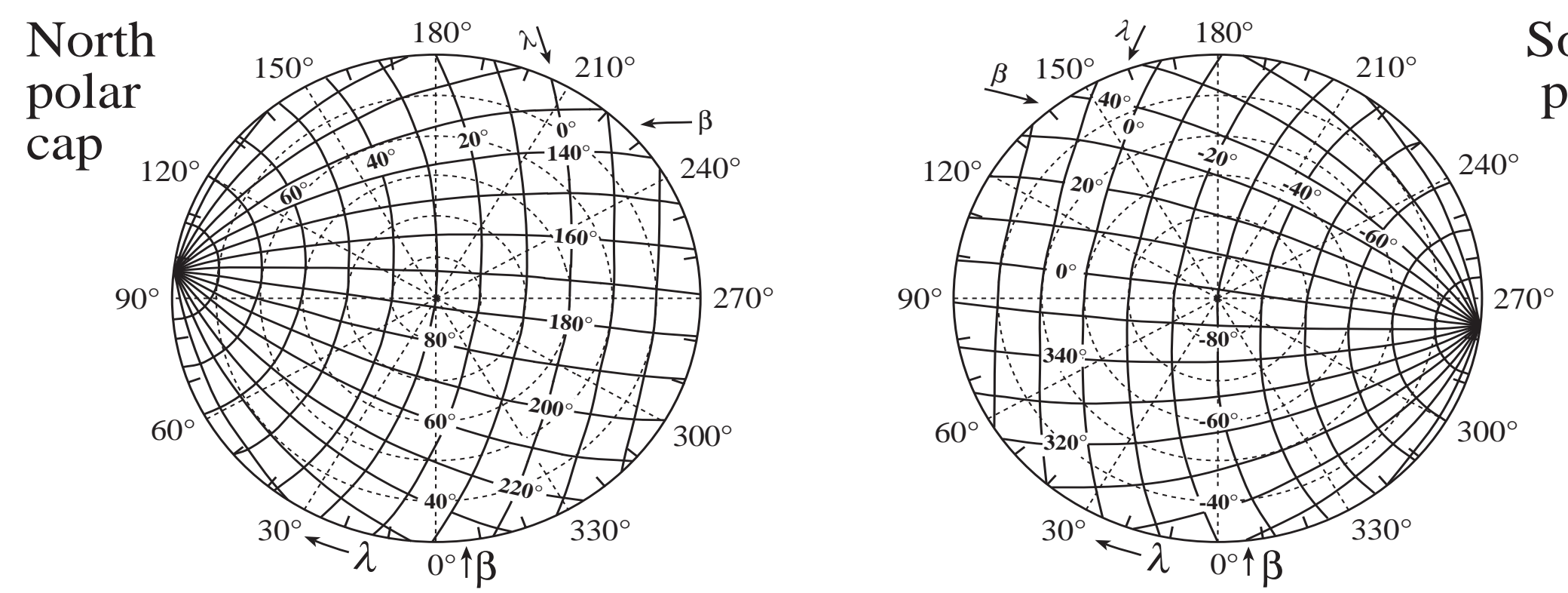

South

polar

cap

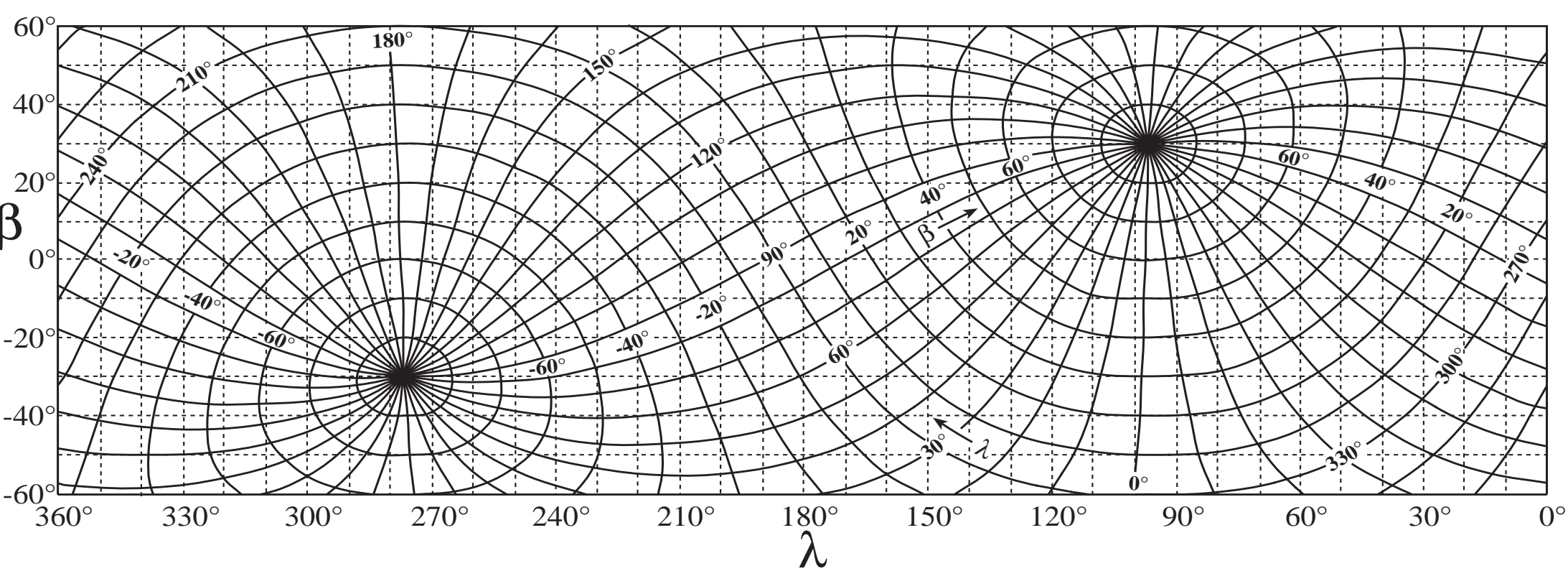




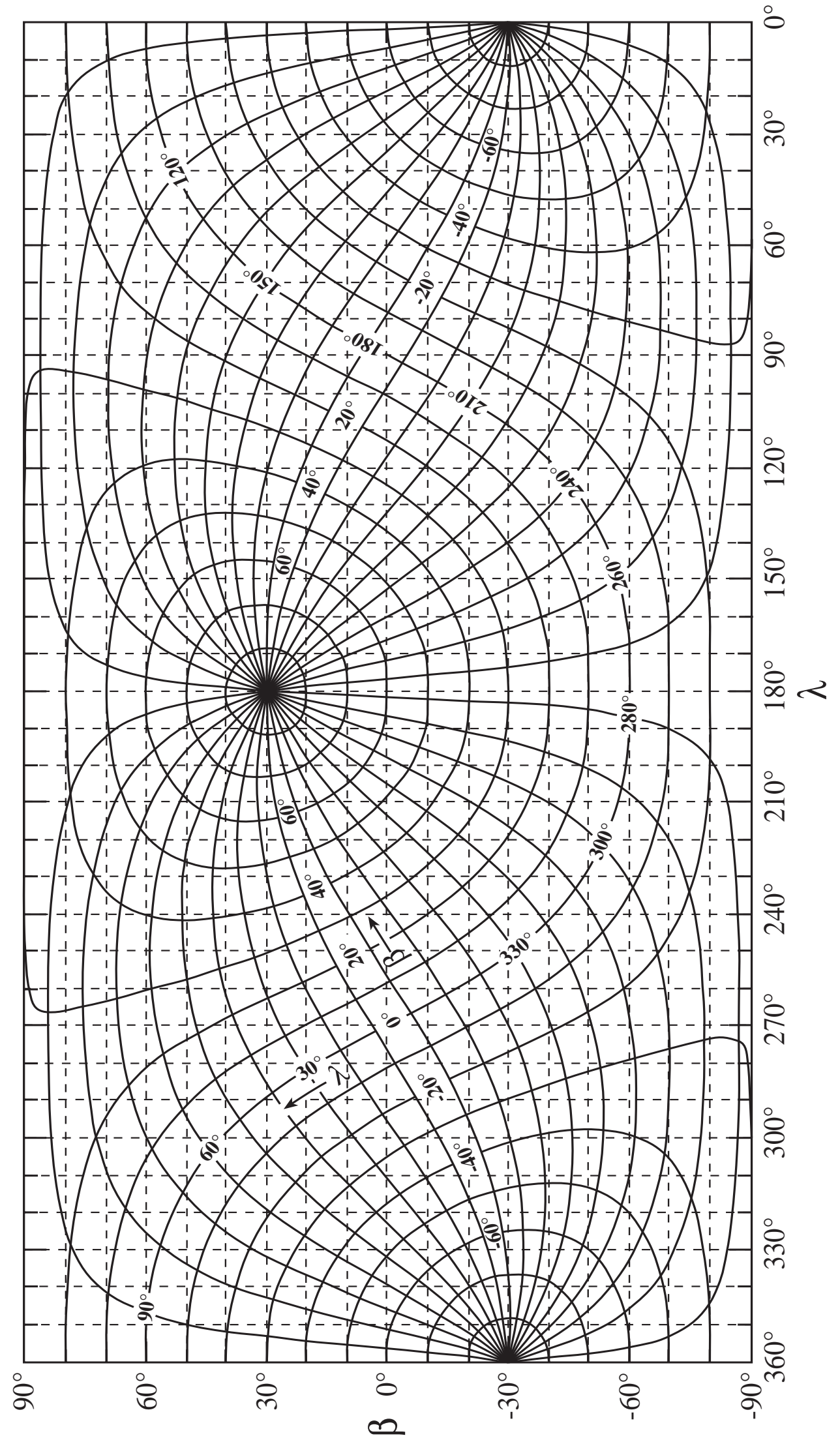

Fig. 7. Relation between coordinates $l, b$ (lines) and $\lambda, \beta$ (underlying dotted grid) for equinox 2000 - alternative projection 
Table 4. Comparison of $B$ and $V$ zenith sky brightnesses at different sites in units of mag/ $\square^{\prime \prime}$. The minimum/maximum values given are averages of the three smallest/largest sky brightness values (nightly averages) given for each site. In the case of ESO and Calar Alto, the numbers in boldface refer to actual $B, V$ measurements, while the numbers in parantheses have been transformed from medium band filter measurements. The given solar $10.7 \mathrm{~cm}$ flux value (in units of $10^{4} \mathrm{Jy}$ ) is the average of the three nights in question

\begin{tabular}{|c|c|c|c|c|c|c|c|}
\hline Site & $\overline{I_{B}(\max )}$ & $I_{B}(\min )$ & $I_{V}(\max )$ & $I_{V}(\min )$ & Solar flux & Corresponding dates & ref. \\
\hline $\mathrm{ESO}$ & $\begin{array}{r}\mathbf{2 2 . 7 4} \\
(22.20)\end{array}$ & $\begin{array}{r}\mathbf{2 2 . 9 7} \\
(22.94)\end{array}$ & $\begin{array}{r}\mathbf{2 1 . 6 9} \\
(20.85)\end{array}$ & $\begin{array}{r}\mathbf{2 1 . 9 1} \\
(22.02)\end{array}$ & $\begin{array}{r}164 \\
168 \\
161 \\
116 \\
162 \\
94\end{array}$ & $\begin{array}{l}\mathbf{7 8 - 0 2 - 0 5} \\
80-12-06 ; 80-06-08 ; 88-12-05 \\
\mathbf{7 8 - 0 2 - 0 8} \\
78-02-08 ; 87-12-16 ; 87-12-19 \\
\mathbf{7 8 - 0 2 - 0 7} \\
87-12-15 ; 87-12-16 ; 87-12-19\end{array}$ & 1. \\
\hline Calar Alto & $\mathbf{2 2 . 5 1}(22.30)$ & $\mathbf{2 3 . 0 5}(22.98)$ & $(21.16)$ & 21.79 & $\begin{array}{r}61 \\
176 \\
206 \\
61\end{array}$ & 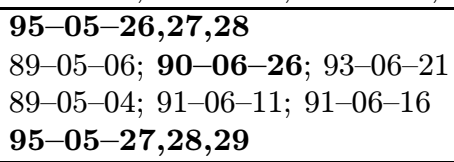 & 2. \\
\hline San Benito Mt. & 22.37 & 23.08 & 21.32 & 22.07 & $\begin{array}{r}233 \\
78 \\
76 \\
\end{array}$ & $\begin{array}{l}80-04-11 ; 81-07-28 ; 82-06-22 \\
76-04-30 ; 87-04-25 ; 87-06-29 \\
76-04-30 ; 87-04-28 ; 87-06-29\end{array}$ & 3. \\
\hline Kitt Peak & 22.65 & 22.98 & 21.60 & 22.01 & $\begin{array}{r}114 \\
75 \\
114 \\
75\end{array}$ & $\begin{array}{l}88-01-21 ; 88-03-17 ; 88-06-14 \\
86-12-02 ; 86-12-30 ; 87-06-22 \\
87-11-20 ; 88-03-17 ; 88-06-14 \\
86-12-02 ; 86-12-31 ; 87-06-22\end{array}$ & 4. \\
\hline Crimea & $\overline{21.91}$ & 23.05 & 21.10 & 22.05 & $\begin{array}{l}122 \\
136\end{array}$ & $\begin{array}{l}68-04-28 ; 71-04-25 ; 70-08-09 \\
68-03-29 ; 68-04-06 ; 68-04-28\end{array}$ & 5. \\
\hline Hawaii & 22.27 & 23.03 & 21.21 & 22.05 & $\begin{array}{l}210 \\
142 \\
166 \\
102\end{array}$ & $\begin{array}{l}88-11-13 ; 89-03-28 ; 89-09-12 \\
87-08-26 ; 87-11-13 ; 89-06-10 \\
85-12-13 ; 88-11-13 ; 89-03-28 \\
86-06-02 ; 87-08-26 ; 88-07-18\end{array}$ & 6. \\
\hline $\begin{array}{l}\text { McDonald } \\
\text { Observatory }\end{array}$ & 22.54 & 23.01 & 21.54 & 21.92 & $\begin{array}{l}138 \\
156 \\
159\end{array}$ & $\begin{array}{l}60-02-04 ; 72-12-30 ; 73-01-12 \\
60-01-27 ; 72-01-11 ; 72-01-15 \\
60-01-27 ; 72-01-15 ; 73-01-08\end{array}$ & 7 . \\
\hline
\end{tabular}

1. Mattila et al. (1996a).

2. Leinert et al. (1995), Leinert et al. (1996, unpublished).

3. Walker (1988).

4. Pilachowski et al. (1989).

5. Lyutyi \& Sharov (1982).

6. Krisciunas (1990).

7. Kalinowski et al. (1975).

\section{Total sky brightness}

In this section we give the minimum diffuse sky brightness to be expected (values for an arbitrary field-of-view have to be estimated as a sum of the components of the night sky brightness). For the ultraviolet and the infrared, extraterrestrial values are given. For the visual spectral region we give the values as seen from ground. Here, the extraterrestrial values would closely correspond to the minimum brightness of the zodiacal light, stars being resolved by optical space telescopes like the HST. For the nearinfrared, sky brightness as seen from ground is also included.

In the infrared, total brightnesses as observed by the DIRBE experiment onboard COBE are conveniently available in the form of weekly averages of the bright- ness seen in different viewing directions from the heliocentric position taken by COBE during the respective week. The data, covering the 10 photometric DIRBE bands from $1.25 \mu \mathrm{m}$ to $240 \mu \mathrm{m}$ (see Sect. 8.5), including Stokes Parameters $Q$ and $U$ for the $1.25 \mu \mathrm{m}, 2.2 \mu \mathrm{m}$ and $3.5 \mu \mathrm{m}$ bands, are available on CD-ROM or tape. Under http://www.gsfc.nasa.gov/astro/cobe/cobe_home.html on the World Wide Web one finds the information necessary to actually receive those data.

\subsection{Ultraviolet}

\subsubsection{Far UV $(91.2 \mathrm{~nm}-180 \mathrm{~nm})$}

The sky brightness over most of this band is the sum of starlight and starlight scattered by interstellar dust. 

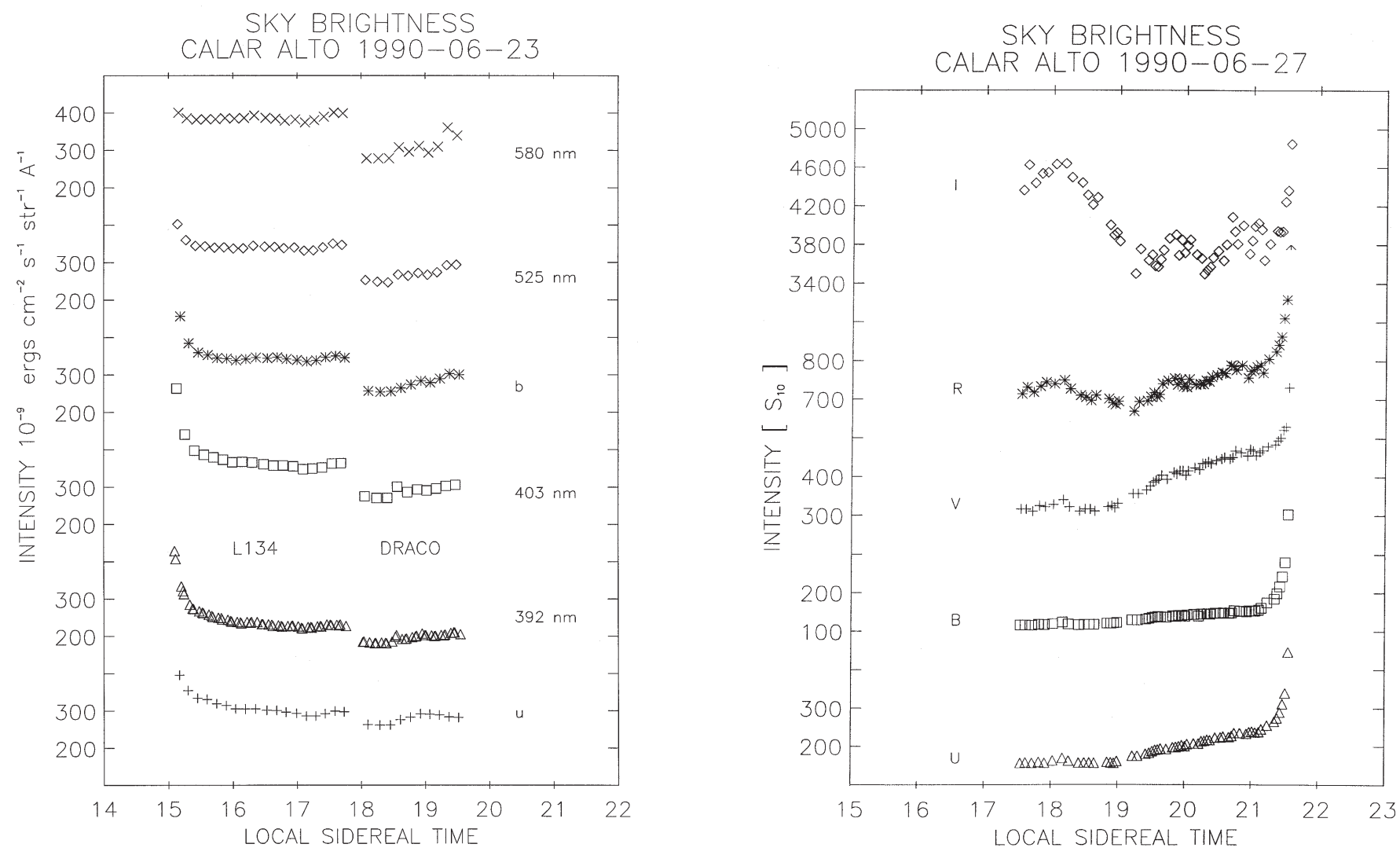

Fig. 8. Variation of the night sky brightness at Calar Alto during the course of one night. Left: Observations in medium band filters, including Strömgren $u$ and $b$ on June 23, 1990. L134 is a dark cloud in Ophiuchus at ecliptic latitude $15^{\circ}$, the Draco field is at high ecliptic latitude, hence the lower brightness level. Right: Observations in broad band filters on June 27, 1990 near the ecliptic pole. - The effect of dawn and dusk can be seen in the data around $15 \mathrm{~h}$ and $21 \mathrm{~h}$ siderial time

The Sun's flux is sufficiently low that zodiacal light is virtually non-existent. An intense diffuse emission in this band is emission from hydrogen Lyman-alpha at $121.6 \mathrm{~nm}$. This flux is produced by scattering of solar radiation by neutral hydrogen in the Earth's geocorona, and by scattering from neutral interstellar hydrogen entering the heliosphere. The geocoronal flux varies by more than a factor of 10 between day and night; typical fluxes range from $3 \mathrm{kR}$ (night) to $34 \mathrm{kR}$ (day). This flux varies with distance from the Earth's geocorona. An excellent exposition of the variation of this flux as a function of these variables is given by Raurden et al. (1986). See also Sect. 6 .

\subsubsection{Near UV $(180 \mathrm{~nm}-300 \mathrm{~nm})$}

The sky brightness in this range is primarily the sum of zodiacal light, starlight, and starlight scattered by interstellar dust. The zodiacal light in this range has not yet been well characterized, the presently available information is shown in Sects. 8.4 and 8.6. The integrated starlight is discussed in Sect. 10.2. Scattering by dust near early type stars is a major contributor to the diffuse flux in this range, and is highly variable from place to place in the Galaxy (see also Sect. 11.5).

\subsection{Visual}

Table 4, adapted from a recent paper (Leinert et al. 1995), gives minimum and maximum values of broadband sky brightness as observed in moonless nights at several observatories in suitable "dark regions" of the sky. The main constituents of this diffuse brightness are airglow, zodiacal light and tropospherically scattered light, in this order, but in roughly comparable quantities. The variation between minimum and maximum is mostly due to solar activity, which leads to increased airglow emission. The individual entries in Table 4 are not stricly comparable. Some of the measurements were performed with small telescopes and excluded stars only down to about 10 mag (San Benito Mt.), about $13 \mathrm{mag}$ (Kitt Peak, $90 \mathrm{~cm}$ telescope, diaphragm 50") and about $12 \mathrm{mag}$ (Hawaii, $15 \mathrm{~cm}$ telescope, $6.5 \square^{\prime}$ ). The residual contributions of individual stars to their observed zenith brightnesses then can be estimated (Roach \& Megill 1961) to be still 0.08-0.22 mag, $0.03-0.11 \mathrm{mag}$, and $0.05-0.15 \mathrm{mag}$, respectively, both at $B$ and $V$. In clear nights therefore the sky appears to be more or less equally dark at all major observatories.

Figure 8 shows the observed variation of sky brightness in a starless spot for a typical night, both for intermediate- 
band and broad-band observations. The central wavelengths of the intermediate bands have been selected to coincide with minima of the night sky spectrum. Figure 9 indicates what emission may be expected outside those bands. Brightness variations usually are well correlated between different wavelength bands (see Leinert et al. 1995 and Fig. 28 in Sect. 6.3). An example for the variation of sky brightness with solar activity is given in Fig. 10 .

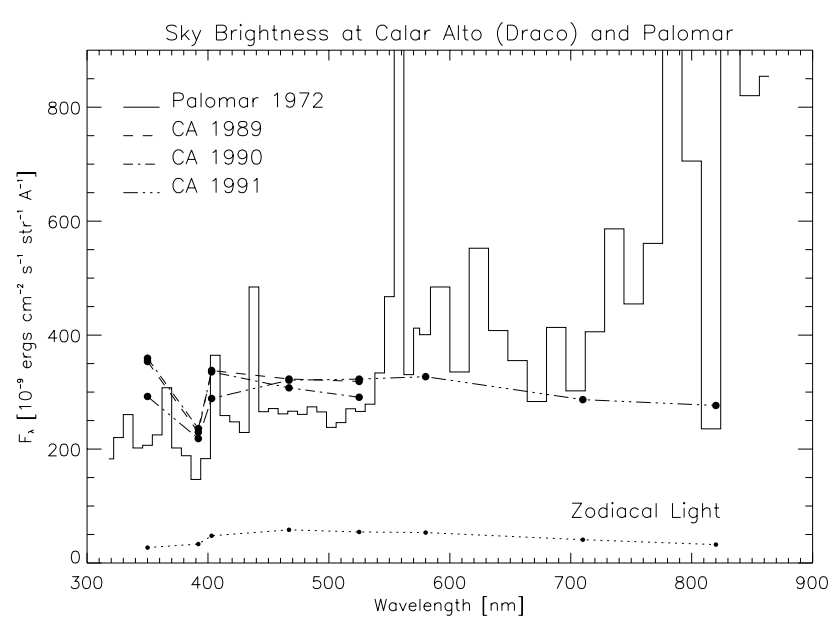

Fig. 9. A low resolution night sky spectrum at Palomar Observatory, taken on November 28, 1972 (Turnrose 1974), compared to medium band measurements on Calar Alto (CA)

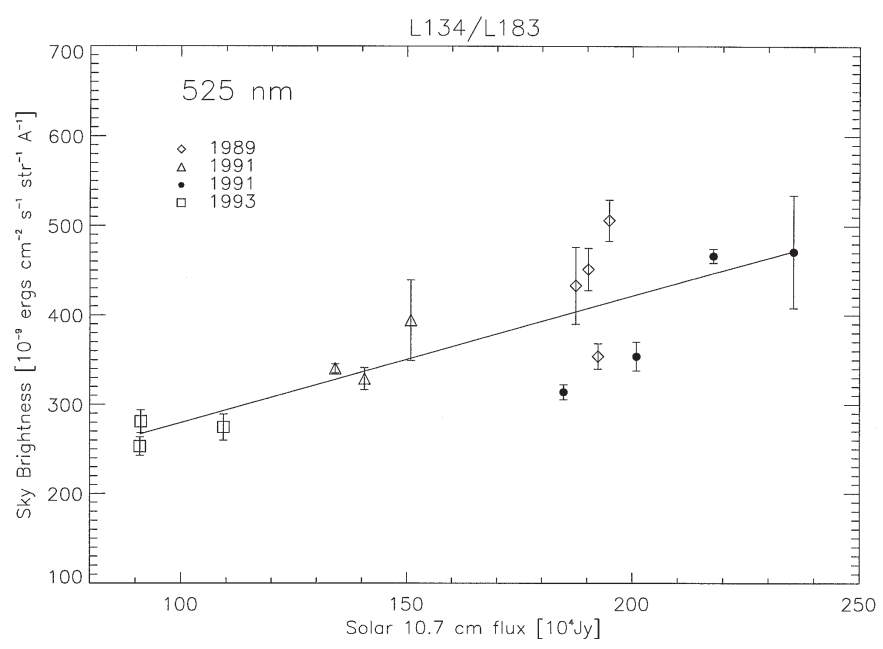

Fig. 10. Correlation between the night sky brightness observed at Calar Alto at $525 \mathrm{~nm}$ with the solar activity, measured by the $10.7 \mathrm{~cm}$ radio flux density (in units of $10^{4} \mathrm{Jy}$ )

\subsection{Near-infrared from the ground}

The near-infrared sky brightness seen from ground at a typical observing site is shown in Fig. 11. Below $2 \mu \mathrm{m}$ the night sky emission is dominated by $\mathrm{OH}$ airglow emission (see also Sect. 6). Above $2 \mu \mathrm{m}$ thermal emission by the atmosphere is dominating. Between $2 \mu \mathrm{m}$ and $4 \mu \mathrm{m}$ emission from the telescope also adds a considerable fraction to the total radiation.

The situation is quite different for observations from Antarctica. The much reduced thermal emission in an environment with winter temperatures below $-60{ }^{\circ} \mathrm{C}$ leads to a substantial reduction of sky background particularly in the $K$ photometric band (Ashley et al. 1996; Nguyen et al. 1996, see Fig. 12 and Table 5). Because of the absence of strong airglow emission between $2.3 \mu \mathrm{m}$ and $2.5 \mu \mathrm{m}$ (see Fig. 27, Sect. 6.1.c), in this spectral region values of zenith sky brightness as low as $50 \mu \mathrm{Jy} \operatorname{arcsec}^{-2}$ ( $K=17.7 \mathrm{mag} \operatorname{arcsec}^{-2}$ ) have been measured. The dependence on zenith distance is normal: proportional to sec $z$ down to $z \approx 50^{\circ}$. In the $L$ band, between $2.9 \mu \mathrm{m}$ and $4.1 \mu \mathrm{m}$, still an improvement by a factor of $40-20$ was found (Ashley et al. 1996).

Table 5. Comparison of $K$ band sky brightnesses ${ }^{a}$

\begin{tabular}{lllccc}
\hline \multicolumn{1}{c}{ Site } & $\begin{array}{c}\lambda \\
(\mu \mathrm{m})\end{array}$ & $\begin{array}{c}\Delta \lambda \\
(\mu \mathrm{m})\end{array}$ & $\begin{array}{c}I_{\nu} \\
\left(\mu \mathrm{Jy} / \square^{\prime \prime}\right)\end{array}$ & $\begin{array}{c}I \\
\left(\mathrm{mag} / \square^{\prime \prime}\right)\end{array}$ & Ref. \\
\hline Mauna Kea & 2.22 & 0.39 & $\approx 4000$ & $\approx 13$ & 1 \\
Mauna Kea & 2.11 & 0.35 & $\approx 2700$ & $\approx 13.4$ & 1 \\
Balloon & 2.4 & 0.1 & $<26$ & $<18.4$ & 2 \\
Balloon & 2.38 & 0.08 & $130 \pm 19$ & 16.7 & 3 \\
South Pole & 2.36 & 0.14 & $162 \pm 67$ & 16.5 & 4 \\
South Pole & 2.40 & 0.04 & 50 & 17.7 & 5 \\
\hline${ }^{a}$ adapted from Nguyen et al. (1996). \\
References: ${ }^{1}$ Wainscoat \& Cowie (1992), ${ }^{2}$ Hofmann et al. \\
(1974), ${ }^{3}$ Matsumoto et al. (1994), ${ }^{4}$ Nguyen et al. (1996), \\
${ }^{5}$ Ashley et al. (1996).
\end{tabular}

\subsection{Infrared}

Table 6 shows the darkest spots on the sky from $1 \mu \mathrm{m}$ to $240 \mu \mathrm{m}$ as measured by the infrared photometric experiment DIRBE on the COBE satellite in an $0.7^{\circ} \times 0.7^{\circ}$ wide field-of-view (adapted from Hauser 1996). These are conservative upper limits to the cosmic infrared background light. For wavelengths of $\lambda \leq 60 \mu \mathrm{m}$, where the zodiacal light (thermal emission) dominates, the darkest fields are close to the ecliptic poles. For longer wavelengths, the thermal emission of interstellar dust is dominating, and the darkest fields are found in regions around the galactic poles with particularly low HI $21 \mathrm{~cm}$ emission (Lockman et al. 1986). 


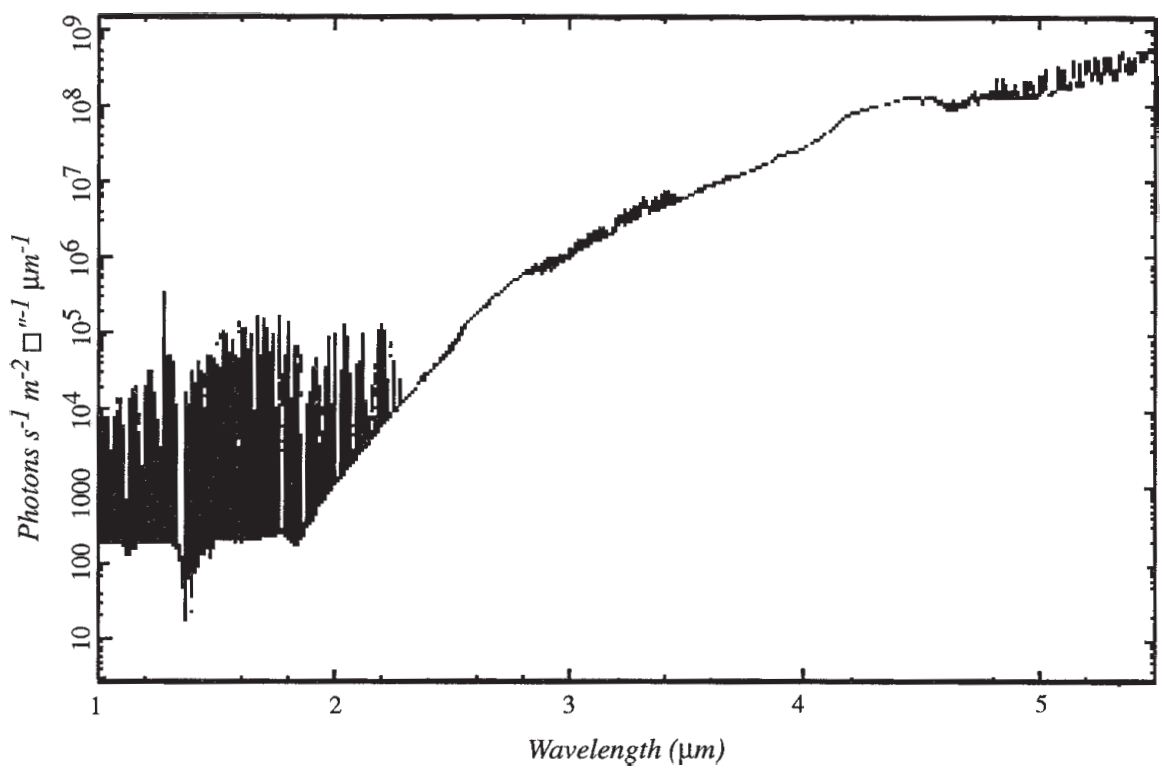

Fig. 11. Near-infrared spectrum of the night sky brightness, measured just inside the cryostat window of the UKIRT IRCAM camera (McCaughrean 1988). Note that $10^{4}$ photons $\mathrm{m}^{-2} \mathrm{~s}^{-1} \square^{\prime \prime-1} \mu \mathrm{m}^{-1}$ correspond to $4.23 \mathrm{Wm}^{-2} \mathrm{sr}^{-1} \mu \mathrm{m}^{-1}$. From Beckwith 1994

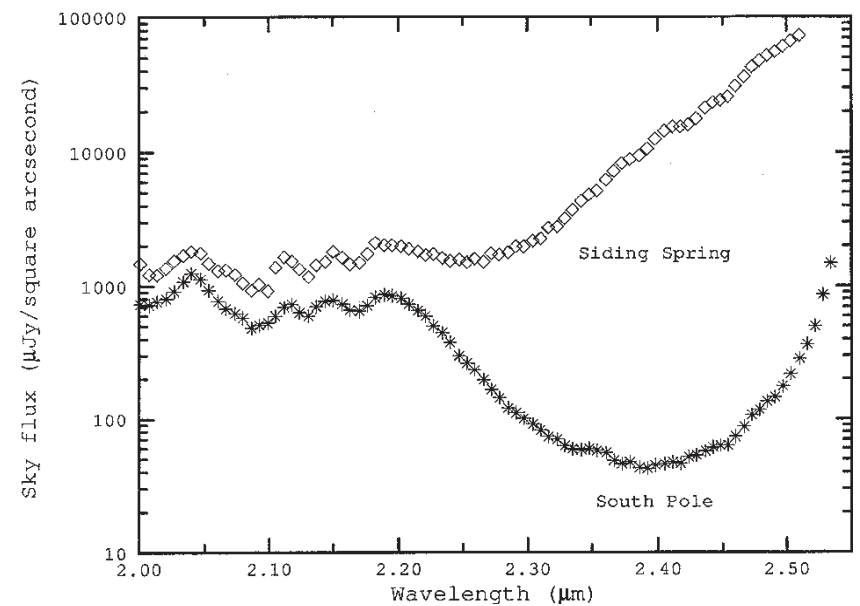

Fig. 12. Near-infrared sky brightness around $2.3 \mu \mathrm{m}$ as observed in Antarctica on May 31, 1994 with an ambient temperature of $-62^{\circ}$. The dip around $2.4 \mu \mathrm{m}$ is due to the lack of airglow emission in this region. The South Pole data are compared to observations obtained at the Siding Spring observatory (Australia) with an ambient temperature of $+10^{\circ}$. From Ashley et al. (1996)
Table 6. Minimum observed sky brightnesses found in the DIRBE weekly averaged sky maps

\begin{tabular}{cccc}
$\lambda$ & $\nu I_{\nu}=\lambda I_{\lambda}$ & $I_{\nu}$ & Reference \\
$(\mu \mathrm{m})$ & $\left(\mathrm{nW} \mathrm{m}^{-2} \mathrm{sr}^{-1}\right)$ & $(\mathrm{MJy} / \mathrm{sr})$ & \\
\hline 1.25 & $393 \pm 13$ & $0.16 \pm 0.005$ & 1 \\
2.2 & $150 \pm 5$ & $0.11 \pm 0.004$ & 1 \\
3.5 & $63 \pm 3$ & $0.074 \pm 0.004$ & 1 \\
4.9 & $192 \pm 7$ & $0.31 \pm 0.01$ & 1 \\
12 & $2660 \pm 310$ & $10.7 \pm 1.2$ & 1 \\
25 & $2160 \pm 330$ & $18 \pm 3$ & 1 \\
60 & $261 \pm 22$ & $5.2 \pm 0.4$ & 1 \\
100 & $74 \pm 10$ & $2.5 \pm 0.3$ & 1 \\
140 & $57 \pm 6$ & $2.7 \pm 0.3$ & 1 \\
240 & $22 \pm 2$ & $1.8 \pm 0.2$ & 1 \\
\hline
\end{tabular}

${ }^{1}$ Hauser (1996). 


\section{Tropospheric scattering}

From earthbound measurements of the night sky brightness the contribution due to tropospheric scattering (see Eq. (1)) has to be subtracted in order to determine its uncontaminated extraterrestrial intensity and polarization. The strongest contributions to scattered light come from airglow, zodiacal light (ZL) and integrated starlight (ISL) - that is, the correction to be applied is in part determined by the brightness distribution of the sources under study themselves. The correction is of the order of $10-100 S_{10}$, which corresponds to $15 \%$ or more of the Zodiacal light, and to typically $10-30 \%$ of the ISL. Due to the limited accuracy to which the correction can be determined, it can be applied explicitly only to measurements aimed at the determination of ZL and ISL. The weaker components of the night sky brightness, DGL and EBL, must be determined by differential methods.

Detailed calculations on first order Rayleigh- and Miescattering (including linear and circular polarization) in the (spherical) Earth's atmosphere illuminated by a uniform, unpolarized source, by the Milky Way and by the Zodiacal light were performed by Staude (1975) for various values of the optical thickness of the Rayleigh and Mie components of the atmosphere, and assuming two different values for the refractive index $m$ of atmospheric aerosols $(m=1.33$, as for water vapour, and $m=1.5-0.1 i$, as for aerosols in dry air). The position and orientation of Milky Way and Zodiacal Light cone were varied independently over the whole range occurring in practice. Some results from this study are reported in the following.

\subsection{A uniform unpolarized source of unit brightness}

The brightness of tropospherically scattered airglow can be estimated using the results obtained for a uniform unpolarized source of unit brightness (extending over the entire visible sky) in the single scattering approximation, which are given in Figs. 13 and 14. They give the intensity of the scattered light and its polarization as a function of zenith distance of the observing direction $z_{0}$, for different values of the zenith extinction $\tau_{\mathrm{R}}$ of the Rayleigh and $\tau_{\mathrm{M}}$ of the Mie component.

Table 7. The correction factors for multiple scattering in a Rayleigh atmosphere for different values of the zenith extinction $\tau_{\mathrm{R}}$. See text for details

\begin{tabular}{|c|c|c|}
\hline$\tau_{\mathrm{R}}$ & $F_{\mathrm{MS}}$ & $f_{\mathrm{MS}}$ \\
\hline 0.05 & $1.12 \pm 0.04$ & $0.95 \pm 0.05$ \\
0.10 & $1.22 \pm 0.06$ & $0.90 \pm 0.05$ \\
0.15 & $1.33 \pm 0.06$ & $0.85 \pm 0.05$ \\
0.20 & $1.44 \pm 0.07$ & $0.80 \pm 0.05$ \\
\hline
\end{tabular}

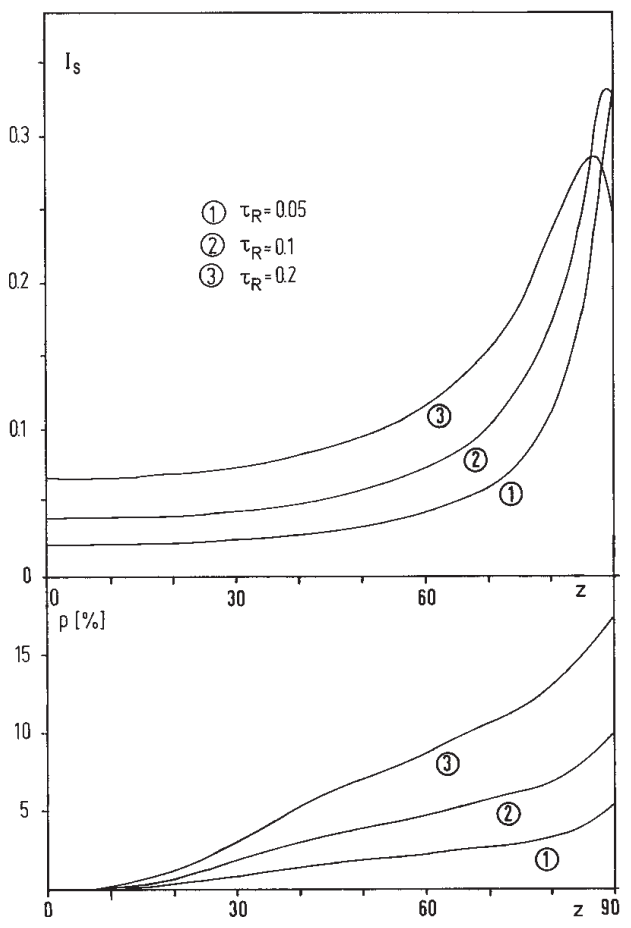

Fig. 13. Intensity and polarization of the atmospheric scattered light in a pure Rayleigh atmosphere, for a source of unit brightness and various values of the zenith extinction $\tau_{\mathrm{R}}$, as a function of zenith distance $z$

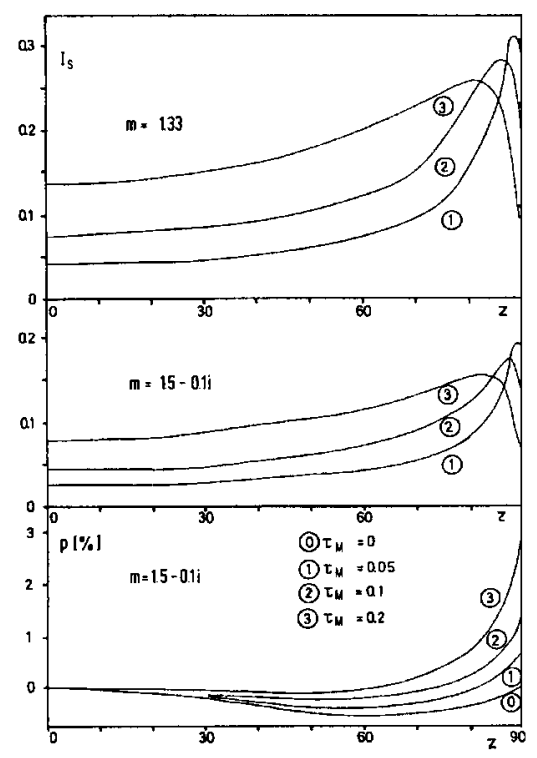

Fig. 14. Same as Fig. 13 for two pure Mie atmospheres 
Table 8. Intensity in $S_{10}$ and polarization of scattered integrated starlight for two pure Rayleigh and two pure Mie atmospheres with the given values of zenith extinction $\tau_{\mathrm{R}}$ and $\tau_{\mathrm{M}}$. The galactic center is assumed at the zenith $(z=0)$, the galactic equator crosses the horizon at $A=90,270 ; l$ and $b$ are galactic coordinates

\begin{tabular}{|c|c|c|c|c||c|c|c|c||c|c|c|c|}
\hline$l$ & $b$ & $A$ & $z$ & $I_{1}$ & $I_{2}$ & $I_{\mathrm{s}}$ & $p_{\mathrm{s}}$ & $\phi$ & $I_{2}$ & $I_{\mathrm{s}}$ & $p_{\mathrm{s}}$ & $\phi$ \\
\hline & & & & \multicolumn{1}{|c||}{} & \multicolumn{3}{|c||}{$\tau_{\mathrm{R}}=0.10$} & \multicolumn{5}{c|}{$\tau_{\mathrm{R}}=0.20$} \\
\hline 0 & 0 & 0 & 0 & 260.0 & 235.3 & 4.0 & 10.4 & 90 & 213.0 & 8.2 & 8.7 & 90 \\
330 & 0 & 90 & 30 & 220.8 & 196.8 & 4.5 & 12.9 & 90 & 175.4 & 9.0 & 11.4 & 90 \\
333 & 14 & 120 & 30 & 112.5 & 100.3 & 4.4 & 11.6 & 69 & 89.4 & 8.9 & 10.2 & 69 \\
344 & 26 & 150 & 30 & 57.3 & 51.1 & 4.4 & 8.3 & 41 & 45.5 & 8.8 & 6.9 & 43 \\
360 & 30 & 180 & 30 & 52.3 & 46.6 & 4.3 & 6.0 & 0 & 41.6 & 8.7 & 4.4 & 0 \\
300 & 0 & 90 & 60 & 151.9 & 124.5 & 7.0 & 18.3 & 90 & 102.0 & 13.4 & 17.4 & 90 \\
304 & 26 & 120 & 60 & 54.1 & 44.3 & 6.8 & 15.9 & 82 & 36.3 & 13.1 & 15.4 & 82 \\
319 & 49 & 150 & 60 & 36.3 & 29.7 & 6.5 & 9.9 & 74 & 24.4 & 12.5 & 10.1 & 77 \\
360 & 60 & 180 & 60 & 29.4 & 24.1 & 6.4 & 4.7 & 90 & 19.7 & 12.2 & 6.1 & 90 \\
\hline & & & & \multicolumn{6}{|c||}{$\tau_{\mathrm{M}}=0.05$} & & \multicolumn{5}{|c|}{$\tau_{\mathrm{M}}=0.15$} & \\
\hline 360 & 0 & 0 & 0 & 260.0 & 247.4 & 5.9 & 0.6 & 90 & 223.9 & 15.8 & 0.5 & 90 \\
330 & 0 & 90 & 30 & 220.8 & 208.4 & 5.8 & 0.7 & 90 & 185.7 & 15.4 & 0.6 & 90 \\
333 & 14 & 120 & 30 & 112.5 & 106.2 & 4.9 & 0.7 & 67 & 94.7 & 13.0 & 0.7 & 68 \\
344 & 26 & 150 & 30 & 57.3 & 54.1 & 3.9 & 0.6 & 40 & 48.2 & 10.2 & 0.6 & 42 \\
360 & 30 & 180 & 30 & 52.3 & 49.4 & 3.5 & 0.5 & 0 & 44.0 & 9.4 & 0.4 & 0 \\
300 & 0 & 90 & 60 & 151.9 & 137.5 & 7.2 & 1.0 & 90 & 112.7 & 17.4 & 1.1 & 90 \\
304 & 26 & 120 & 60 & 54.1 & 48.9 & 5.1 & 1.1 & 86 & 40.1 & 12.3 & 1.3 & 87 \\
319 & 49 & 150 & 60 & 36.3 & 32.8 & 3.5 & 0.9 & 86 & 26.9 & 8.6 & 1.1 & 88 \\
360 & 60 & 180 & 60 & 29.4 & 26.6 & 3.0 & 0.7 & 90 & 21.8 & 7.4 & 1.0 & 90 \\
\hline
\end{tabular}

Table 9. Same as for Table 8, but with the Galactic center at $A=0, z=180$

\begin{tabular}{|c|c|c|c|c|c|c|c|c|c|c|c|c|}
\hline$l$ & $b$ & $\bar{A}$ & $z$ & $I_{1}$ & $I_{2}$ & $I_{\mathrm{s}}$ & $p_{\mathrm{s}}$ & $\phi$ & $I_{2}$ & $I_{\mathrm{s}}$ & $p_{\mathrm{S}}$ & $\phi$ \\
\hline & & & & & \multicolumn{4}{|c|}{$\tau_{\mathrm{R}}=0.10$} & \multicolumn{4}{|c|}{$\tau_{\mathrm{R}}=0.20$} \\
\hline 180 & 0 & 0 & 0 & 100.0 & 90.5 & 2.8 & 9.9 & 90 & 81.9 & 5.6 & 8.1 & 90 \\
\hline 180 & 30 & 0 & 30 & 50.6 & 45.1 & 3.1 & 7.0 & 180 & 40.2 & 6.1 & 5.2 & 180 \\
\hline 196 & 26 & 30 & 30 & 51.9 & 46.2 & 3.1 & 8.0 & 144 & 41.2 & 6.2 & 6.5 & 142 \\
\hline 207 & 14 & 60 & 30 & 67.6 & 60.2 & 3.1 & 9.8 & 115 & 53.7 & 6.2 & 8.5 & 114 \\
\hline 210 & 0 & 90 & 30 & 100.1 & 89.2 & 3.2 & 10.6 & 90 & 79.5 & 6.3 & 9.3 & 90 \\
\hline 180 & 60 & 0 & 60 & 29.0 & 23.8 & 4.7 & 0.6 & 180 & 19.5 & 8.9 & 1.5 & 90 \\
\hline 221 & 49 & 30 & 60 & 35.8 & 29.3 & 4.8 & 5.4 & 120 & 24.1 & 9.1 & 5.8 & 111 \\
\hline 236 & 26 & 60 & 60 & 51.9 & 42.5 & 5.0 & 10.2 & 103 & 34.9 & 9.5 & 10.2 & 101 \\
\hline \multirow[t]{2}{*}{240} & 0 & 90 & 60 & 101.8 & 83.4 & 5.1 & 12.1 & 90 & 68.4 & 9.7 & 12.0 & 90 \\
\hline & & & & & \multicolumn{4}{|c|}{$\tau_{\mathrm{M}}=0.05$} & \multicolumn{4}{|c|}{$\tau_{\mathrm{M}}=0.15$} \\
\hline 180 & 0 & 0 & 0 & 100.0 & 95.1 & 3.0 & 0.7 & 90 & 86.1 & 8.0 & 0.6 & 90 \\
\hline 180 & 30 & 0 & 30 & 50.6 & 47.7 & 2.5 & 0.7 & 180 & 42.5 & 6.5 & 0.6 & 180 \\
\hline 196 & 26 & 30 & 30 & 51.9 & 49.0 & 2.6 & 0.7 & 148 & 43.6 & 6.9 & 0.6 & 146 \\
\hline 207 & 14 & 60 & 30 & 67.6 & 63.8 & 3.0 & 0.6 & 118 & 56.8 & 7.9 & 0.6 & 116 \\
\hline 210 & 0 & 90 & 30 & 100.1 & 94.5 & 3.3 & 0.6 & 90 & 84.3 & 8.7 & 0.6 & 90 \\
\hline 180 & 60 & 0 & 60 & 29.0 & 26.2 & 2.6 & 0.2 & 180 & 21.5 & 6.3 & 0 & 90 \\
\hline 221 & 49 & 30 & 60 & 35.8 & 32.4 & 3.0 & 0.2 & 129 & 26.6 & 7.2 & 0.3 & 107 \\
\hline 236 & 26 & 60 & 60 & 51.9 & 47.0 & 4.0 & 0.5 & 101 & 38.5 & 9.6 & 0.6 & 98 \\
\hline 240 & 0 & 90 & 60 & 101.8 & 92.1 & 5.1 & 0.6 & 90 & 75.5 & 12.3 & 0.6 & 90 \\
\hline
\end{tabular}


Table 10. Intensity in $S_{10}$ and polarization of tropospherically scattered Zodiacal light. The Sun is located at $A=90, z=105$, the ecliptic is perpendicular to the horizon

\begin{tabular}{|c|c|c|c|c|c|c|c|c|c|c|c|c|}
\hline$\epsilon$ & $\beta$ & $A$ & $\bar{z}$ & $I_{1}$ & $I_{2}$ & $\overline{I_{\mathrm{s}}}$ & $p_{\mathrm{S}}$ & $\bar{\phi}$ & $I_{2}$ & $\overline{I_{\mathrm{s}}}$ & $p_{\mathrm{s}}$ & $\phi$ \\
\hline & & & & & \multicolumn{4}{|c|}{$\tau_{\mathrm{R}}=0.10$} & \multicolumn{4}{|c|}{$\tau_{\mathrm{R}}=0.20$} \\
\hline 105 & 0 & 0 & 0 & 158.2 & 143.2 & 9.5 & 32.5 & 90 & 129.6 & 18.5 & 27.6 & 90 \\
\hline 85 & 0 & 90 & 20 & 220.3 & 198.1 & 10.6 & 26.5 & 90 & 178.2 & 20.5 & 22.6 & 90 \\
\hline 89 & 13 & 130 & 20 & 173.1 & 155.7 & 10.3 & 27.8 & 48 & 140.0 & 20.0 & 23.5 & 48 \\
\hline 105 & 20 & 180 & 20 & 133.6 & 120.1 & 9.9 & 31.6 & 178 & 108.0 & 19.1 & 26.5 & 177 \\
\hline 125 & 0 & 270 & 20 & 138.6 & 124.7 & 10.0 & 33.2 & 90 & 112.1 & 19.3 & 28.6 & 90 \\
\hline 65 & 0 & 90 & 40 & 351.6 & 308.7 & 13.7 & 18.7 & 90 & 271.0 & 25.9 & 16.6 & 69 \\
\hline 72 & 24 & 130 & 40 & 170.2 & 149.5 & 12.9 & 20.2 & 46 & 131.2 & 24.3 & 17.1 & 47 \\
\hline 105 & 40 & 180 & 40 & 102.4 & 89.9 & 11.4 & 29.0 & 175 & 78.9 & 21.6 & 23.8 & 174 \\
\hline 145 & 0 & 270 & 40 & 146.8 & 128.9 & 12.7 & 27.9 & 90 & 113.2 & 23.9 & 25.1 & 90 \\
\hline 45 & 0 & 90 & 60 & 865.6 & 709.4 & 21.4 & 13.0 & 90 & 581.4 & 38.5 & 12.5 & 90 \\
\hline 52 & 34 & 130 & 60 & 186.8 & 153.1 & 19.2 & 10.8 & 44 & 125.5 & 34.6 & 9.1 & 48 \\
\hline 105 & 60 & 180 & 60 & 90.5 & 74.2 & 15.8 & 25.9 & 171 & 60.8 & 28.6 & 20.5 & 170 \\
\hline 165 & 0 & 270 & 60 & 164.4 & 134.8 & 20.0 & 20.1 & 90 & 110.4 & 35.9 & 19.2 & 90 \\
\hline 30 & 0 & 90 & 75 & 2200.0 & 1504.5 & 37.8 & 11.3 & 90 & 1028.9 & 61.6 & 11.7 & 90 \\
\hline 34 & 38 & 130 & 75 & 201.4 & 137.7 & 33.1 & 3.0 & 45 & 94.2 & 54.1 & 3.1 & 66 \\
\hline 105 & 75 & 180 & 75 & 78.8 & 53.9 & 26.4 & 23.9 & 169 & 36.9 & 43.3 & 18.2 & 166 \\
\hline \multirow[t]{2}{*}{180} & 0 & 270 & 75 & 180.0 & 123.1 & 36.4 & 15.2 & 90 & 84.2 & 59.3 & 15.4 & 90 \\
\hline & & & & & \multicolumn{4}{|c|}{$\tau_{\mathrm{M}}=0.05$} & \multicolumn{4}{|c|}{$\tau_{\mathrm{M}}=0.15$} \\
\hline 105 & 0 & 0 & 0 & $\begin{array}{l}158.2 \\
\end{array}$ & 150.5 & 6.5 & 17.6 & 90 & $\begin{array}{l}136.2 \\
\end{array}$ & 17.3 & 17.3 & 90 \\
\hline 85 & 0 & 90 & 20 & 220.3 & 208.9 & 8.9 & 18.9 & 90 & 187.9 & 23.4 & 18.8 & 90 \\
\hline 89 & 13 & 130 & 20 & 173.1 & 164.2 & 7.9 & 18.8 & 52 & 147.6 & 20.8 & 18.7 & 52 \\
\hline 105 & 20 & 180 & 20 & 133.6 & 126.7 & 6.3 & 17.4 & 4 & 113.9 & 16.7 & 17.2 & 4 \\
\hline 125 & 0 & 270 & 20 & 138.6 & 131.5 & 5.9 & 12.8 & 90 & 118.2 & 15.6 & 12.7 & 90 \\
\hline 65 & 0 & 90 & 40 & 351.6 & 329.4 & 16.0 & 17.5 & 90 & 289.2 & 40.4 & 17.6 & 90 \\
\hline 72 & 24 & 130 & 40 & 170.2 & 159.5 & 10.9 & 17.9 & 52 & 140.0 & 27.6 & 18.0 & 52 \\
\hline 105 & 40 & 180 & 40 & 102.4 & 95.9 & 6.5 & 17.1 & 7 & 84.2 & 16.6 & 16.9 & 7 \\
\hline 145 & 0 & 270 & 40 & 146.8 & 137.6 & 6.8 & 6.7 & 90 & 120.8 & 17.6 & 6.7 & 90 \\
\hline 45 & 0 & 90 & 60 & 865.6 & 783.6 & 38.4 & 14.8 & 90 & 642.2 & 88.6 & 15.1 & 90 \\
\hline 52 & 34 & 130 & 60 & 186.8 & 169.1 & 17.5 & 15.3 & 50 & 138.6 & 40.7 & 15.6 & 51 \\
\hline 105 & 60 & 180 & 60 & 90.5 & 81.9 & 7.9 & 16.6 & 10 & 67.1 & 18.8 & 16.3 & 10 \\
\hline 165 & 0 & 270 & 60 & 164.4 & 148.9 & 9.9 & 9.0 & 90 & 122.0 & 23.6 & 3.1 & 90 \\
\hline 30 & 0 & 90 & 75 & 2200.0 & 1819.3 & 92.3 & 12.7 & 90 & 1244.2 & 178.9 & 13.2 & 90 \\
\hline 34 & 38 & 139 & 75 & 201.4 & 166.5 & 29.8 & 12.7 & 49 & 113.9 & 59.1 & 13.0 & 50 \\
\hline 105 & 75 & 180 & 75 & 78.8 & 65.2 & 11.5 & 16.0 & 12 & 44.6 & 23.7 & 15.5 & 12 \\
\hline 180 & 0 & 270 & 75 & 180.0 & 148.9 & 16.1 & 2.1 & 90 & 101.8 & 32.7 & 2.5 & 90 \\
\hline
\end{tabular}

The influence of multiple Rayleigh scattering was estimated using the work of Dave (1964) and of de Bary \& Bullrich (1964), who determined the higher order contributions to the scattered light from a point source in a plane-parallel atmosphere. The derived correction factors $F_{\mathrm{MS}}=I_{\mathrm{MS}} / I_{\mathrm{SS}}$ for the intensity, and $f_{\mathrm{MS}}=p_{\mathrm{MS}} / p_{\mathrm{SS}}$ for the depolarization of scattered light are given in Table 7 . All results for Rayleigh scattering given in the following are corrected for multiple scattering. For Mie scattering, de Bary (1964) concludes that higher order contributions are negligible for scattering angles $\theta<30^{\circ}$. Therefore, since the main contribution by atmospheric aerosols to the scattered light comes from regions with $\theta<30^{\circ}$, no corrections were applied to the first order results for Mie scattering.

\subsection{The integrated starlight}

The integrated starlight scattered in the troposphere was calculated using an analytical model for the extraterrestrial brightness of the ISL: a two dimensional Gauss distribution was fitted to the blue isophotes given by Elsässer \& Haug (1960). The constants were adjusted to give a model intensity $I_{1}(l=0, b=0)=260 S_{10}, I_{1}(l=120, b=0)=$ $I_{1}(l=240, b=0)=100 S_{10}$, and $I_{1}(l, b= \pm 30)=50$ $S_{10}$. At higher galactic latitudes an exponential decrease was assumed, with $I_{1}(l, b= \pm 80)=20 S_{10}$, following the 
Table 11. Same as Table 10, with the Sun at $A=90, z=135$

\begin{tabular}{|c|c|c|c|c|c|c|c|c|c|c|c|c|}
\hline$\epsilon$ & $\beta$ & $A$ & $z$ & $\overline{I_{1}}$ & $\overline{I_{2}}$ & $I_{\mathrm{S}}$ & $p_{\mathrm{s}}$ & $\phi$ & $I_{2}$ & $\overline{I_{\mathrm{S}}}$ & $p_{\mathrm{s}}$ & $\phi$ \\
\hline & & & & & \multicolumn{4}{|c|}{$\tau_{\mathrm{R}}=0.10$} & \multicolumn{4}{|c|}{$\tau_{\mathrm{R}}=0.20$} \\
\hline 135 & 0 & 0 & 0 & 141.1 & 127.7 & 6.6 & 16.9 & 90 & 115.6 & 13.1 & 14.3 & 90 \\
\hline 115 & 0 & 90 & 20 & 143.2 & 128.8 & 7.0 & 17.8 & 90 & 115.9 & 13.7 & 15.3 & 90 \\
\hline 119 & 13 & 130 & 20 & 128.4 & 115.5 & 6.9 & 17.1 & 53 & 103.9 & 13.6 & 14.5 & 53 \\
\hline 135 & 20 & 180 & 20 & 120.2 & 108.1 & 6.9 & 15.2 & 3 & 97.3 & 13.6 & 12.6 & 3 \\
\hline 155 & 0 & 270 & 20 & 153.6 & 138.1 & 7.2 & 14.7 & 90 & 124.2 & 14.1 & 12.6 & 90 \\
\hline 95 & 0 & 90 & 40 & 183.5 & 161.1 & 8.4 & 17.8 & 90 & 141.4 & 16.2 & 16.0 & 90 \\
\hline 102 & 24 & 130 & 40 & 129.7 & 113.9 & 8.2 & 16.1 & 57 & 100.0 & 15.8 & 14.1 & 59 \\
\hline 135 & 40 & 180 & 40 & 92.9 & 81.6 & 8.0 & 11.8 & 7 & 71.6 & 15.4 & 9.1 & 8 \\
\hline 175 & 0 & 270 & 40 & 176.6 & 155.0 & 8.8 & 13.1 & 90 & 136.1 & 17.0 & 11.9 & 90 \\
\hline 75 & 0 & 90 & 60 & 273.4 & 224.1 & 12.6 & 16.6 & 90 & 183.6 & 23.1 & 15.8 & 90 \\
\hline 82 & 34 & 130 & 60 & 128.5 & 105.3 & 11.9 & 13.6 & 63 & 86.3 & 21.9 & 12.8 & 66 \\
\hline 135 & 60 & 180 & 60 & 90.0 & 73.8 & 11.1 & 7.7 & 16 & 60.4 & 20.5 & 5.2 & 22 \\
\hline 195 & 0 & 270 & 60 & 164.4 & 134.8 & 13.1 & 12.5 & 90 & 110.4 & 24.0 & 12.1 & 90 \\
\hline 60 & 0 & 90 & 75 & 420.0 & 287.2 & 22.1 & 15.3 & 90 & 196.4 & 37.0 & 15.3 & 90 \\
\hline 64 & 36 & 130 & 75 & 137.9 & 94.3 & 20.6 & 11.1 & 70 & 64.5 & 34.5 & 11.4 & 74 \\
\hline 135 & 75 & 180 & 75 & 78.9 & 53.9 & 18.5 & 5.8 & 27 & 36.9 & 31.2 & 4.1 & 42 \\
\hline \multirow[t]{2}{*}{210} & 0 & 270 & 75 & 150.0 & 102.6 & 22.7 & 12.9 & 90 & 70.2 & 37.9 & 13.1 & 90 \\
\hline & & & & & \multicolumn{4}{|c|}{$\tau_{\mathrm{M}}=0.05$} & \multicolumn{4}{|c|}{$\tau_{\mathrm{M}}=0.15$} \\
\hline 135 & 0 & 0 & 0 & 141.1 & 134.2 & 5.4 & 8.2 & 90 & 121.5 & 14.4 & 8.1 & 90 \\
\hline 115 & 0 & 90 & 20 & 143.2 & 135.8 & 5.9 & 13.8 & 90 & 122.2 & 15.6 & 13.6 & 90 \\
\hline 119 & 13 & 130 & 20 & 128.4 & 121.8 & 5.6 & 12.8 & 57 & 109.5 & 14.8 & 12.6 & 57 \\
\hline 135 & 20 & 180 & 20 & 120.2 & 114.0 & 5.2 & 8.8 & 15 & 102.6 & 13.9 & 8.7 & 15 \\
\hline 155 & 0 & 270 & 20 & 153.6 & 145.6 & 5.7 & 3.1 & 90 & 131.0 & 15.1 & 3.0 & 90 \\
\hline 95 & 0 & 90 & 40 & 183.5 & 171.9 & 8.3 & 17.3 & 90 & 151.0 & 21.1 & 17.3 & 90 \\
\hline 102 & 24 & 130 & 40 & 129.7 & 121.5 & 6.9 & 16.1 & 61 & 106.7 & 17.6 & 19.0 & 62 \\
\hline 135 & 40 & 180 & 40 & 92.9 & 87.1 & 5.5 & 10.4 & 28 & 76.5 & 14.1 & 10.3 & 28 \\
\hline 175 & 0 & 270 & 40 & 176.6 & 165.4 & 7.0 & 0.9 & 90 & 145.2 & 18.1 & 0.9 & 90 \\
\hline 75 & 0 & 90 & 60 & 273.4 & 247.5 & 14.9 & 18.5 & 90 & 202.9 & 34.8 & 18.6 & 90 \\
\hline 82 & 34 & 130 & 60 & 128.5 & 116.3 & 10.0 & 17.6 & 64 & 95.4 & 23.6 & 17.6 & 64 \\
\hline 135 & 60 & 180 & 60 & 90.0 & 81.5 & 6.9 & 12.5 & 38 & 66.8 & 16.5 & 12.3 & 38 \\
\hline 195 & 0 & 270 & 60 & 164.4 & 148.9 & 9.6 & 0.7 & 90 & 122.0 & 23.0 & 0.7 & 90 \\
\hline 60 & 0 & 90 & 75 & 420.0 & 347.3 & 29.3 & 18.4 & 90 & 237.5 & 57.6 & 18.5 & 90 \\
\hline 64 & 38 & 130 & 75 & 137.9 & 114.0 & 16.4 & 17.5 & 65 & 78.0 & 32.9 & 17.6 & 65 \\
\hline 135 & 75 & 180 & 75 & 78.9 & 65.2 & 10.1 & 13.7 & 42 & 44.6 & 20.8 & 13.5 & 42 \\
\hline 210 & 0 & 270 & 75 & 150.0 & 124.0 & 14.4 & 1.7 & 90 & 84.8 & 29.6 & 1.7 & 90 \\
\hline
\end{tabular}

star counts of Roach \& Megill (1961). The assumption of such a smooth brightness distribution is safe even for Mie scattering, since also in this case scattering angles up to $\theta=30^{\circ}$ contribute substantially to the integrated scattered light. Figure 15 shows the intensity of the scattered ISL as a function of zenith distance for the case that the galactic centre is at the zenith. In Table 8 the scattered intensity $I_{\mathrm{s}}$, and its degree and orientation of polarization $p_{\mathrm{s}}$ (in percent) and $\phi$ are tabulated for this situation together with the assumed source brightness $I_{1}$ in the viewing direction and the transmitted brightness $I_{2}$ weakened by atmospheric extinction. In Table 9 the same values are given for the galactic anticentre at the zenith. The refractive index of the Mie particles is assumed to be $m=1.33$.

\subsection{The Zodiacal light}

Intensity and polarization of Zodiacal light scattered in the troposphere were calculated assuming the brightness distribution given by Dumont (1965) at $\lambda=5000 \AA$. For the linear polarization the values measured by Weinberg (1964) at the ecliptic were used, assuming that over the whole sky the polarization is a function of angular distance 
Table 12. Same as Tables 10 and 11 , with the Sun at $A=90, z=180$

\begin{tabular}{|c|c|c|c|c||c|c|c|c||c|c|c|c|}
\hline$\epsilon$ & $\beta$ & $A$ & $z$ & $I_{1}$ & $I_{2}$ & $I_{\mathrm{s}}$ & $p_{\mathrm{s}}$ & $\phi$ & $I_{2}$ & $I_{\mathrm{s}}$ & $p_{\mathrm{s}}$ & $\phi$ \\
\hline & & & & \multicolumn{1}{|c||}{} & \multicolumn{3}{|c||}{$\tau_{\mathrm{R}}=0.10$} & \multicolumn{3}{|c|}{$\tau_{\mathrm{R}}=0.20$} \\
\hline 180 & 0 & 0 & 0 & 180.0 & 162.9 & 6.0 & 5.9 & 90 & 147.4 & 12.0 & 4.8 & 90 \\
160 & 0 & 90 & 20 & 158.0 & 142.1 & 6.3 & 7.0 & 90 & 127.8 & 12.5 & 6.0 & 90 \\
164 & 13 & 130 & 20 & 144.5 & 129.9 & 6.3 & 6.0 & 57 & 116.9 & 12.5 & 5.0 & 58 \\
180 & 20 & 180 & 20 & 130.0 & 116.9 & 6.2 & 4.2 & 0 & 105.1 & 12.4 & 3.2 & 0 \\
140 & 0 & 90 & 40 & 144.0 & 126.4 & 7.4 & 10.1 & 90 & 111.0 & 14.4 & 9.2 & 90 \\
147 & 24 & 130 & 40 & 117.4 & 103.1 & 7.3 & 7.7 & 71 & 90.5 & 14.2 & 7.1 & 74 \\
180 & 40 & 180 & 40 & 90.0 & 79.0 & 7.2 & 0.3 & 87 & 69.4 & 14.0 & 1.3 & 89 \\
120 & 0 & 90 & 60 & 140.0 & 114.7 & 10.5 & 13.8 & 90 & 94.0 & 19.6 & 13.2 & 90 \\
127 & 34 & 130 & 60 & 101.9 & 83.5 & 10.3 & 11.2 & 82 & 68.4 & 19.2 & 11.1 & 83 \\
180 & 60 & 180 & 60 & 90.0 & 73.8 & 9.9 & 6.2 & 90 & 60.4 & 18.6 & 7.1 & 90 \\
105 & 0 & 90 & 75 & 158.2 & 108.2 & 17.9 & 16.0 & 90 & 74.0 & 30.4 & 15.8 & 90 \\
109 & 36 & 130 & 75 & 102.3 & 70.0 & 17.4 & 13.7 & 86 & 47.9 & 29.6 & 13.9 & 87 \\
180 & 75 & 180 & 75 & 78.9 & 53.9 & 16.7 & 9.9 & 90 & 36.9 & 28.4 & 10.9 & 90 \\
\hline & & & & \multicolumn{1}{|c||}{} & \multicolumn{3}{|c||}{$\tau_{\mathrm{M}}=0.05$} & & & $\tau_{\mathrm{M}}=0.15$ & \\
\hline 180 & 0 & 0 & 0 & 180.0 & 171.2 & 5.7 & 0.7 & 90 & 155.0 & 15.2 & 0.6 & 90 \\
160 & 0 & 90 & 20 & 158.0 & 149.8 & 5.7 & 1.6 & 90 & 134.7 & 15.1 & 1.5 & 90 \\
164 & 13 & 130 & 20 & 144.5 & 137.0 & 5.5 & 1.2 & 72 & 123.2 & 14.7 & 1.2 & 73 \\
180 & 20 & 180 & 20 & 130.0 & 123.3 & 5.3 & 0.1 & 88 & 110.9 & 14.1 & 0.2 & 88 \\
140 & 0 & 90 & 40 & 144.0 & 134.9 & 6.5 & 5.5 & 90 & 118.5 & 16.7 & 5.3 & 90 \\
147 & 24 & 130 & 40 & 117.4 & 110.0 & 5.9 & 4.8 & 85 & 96.6 & 15.2 & 4.7 & 85 \\
180 & 40 & 180 & 40 & 90.0 & 84.3 & 5.3 & 3.8 & 90 & 74.0 & 13.7 & 3.8 & 90 \\
120 & 0 & 90 & 60 & 140.0 & 126.7 & 8.9 & 10.7 & 90 & 103.9 & 21.3 & 10.5 & 90 \\
127 & 34 & 130 & 60 & 101.9 & 92.2 & 7.6 & 10.0 & 88 & 75.6 & 18.2 & 9.9 & 88 \\
180 & 60 & 180 & 60 & 90.0 & 81.5 & 6.6 & 9.2 & 90 & 66.8 & 15.9 & 9.1 & 90 \\
105 & 0 & 90 & 75 & 158.2 & 130.8 & 14.4 & 13.9 & 90 & 89.5 & 29.2 & 13.7 & 90 \\
109 & 38 & 130 & 75 & 102.3 & 84.6 & 11.7 & 13.2 & 89 & 57.9 & 23.9 & 13.0 & 89 \\
180 & 75 & 180 & 75 & 78.9 & 65.2 & 9.7 & 12.4 & 90 & 44.6 & 20.0 & 12.3 & 90 \\
\hline
\end{tabular}

to the Sun (=elongation $\epsilon$, see Sect. 3.5) alone (Dumont $\&$ Sanchez Martinez 1966). The polarization was assumed to be perpendicular to the direction of the Sun.

Figures 16 and 17 show the results for two cases, pure Rayleigh- and pure Mie-scattering (water vapor), respectively. In Tables 10, 11 and 12 the results are collected for three different positions of the Sun below the horizon. The ecliptic is assumed to be perpendicular to the horizon. All other quantities as in Tables 8 and 9.

\section{Airglow}

The airglow emissions vary considerably with time, on short (minutes) and long timescales, mainly due to changes in the atmosphere and in solar activity. They also depend on geomagnetic latitude, with a distinctive tropical brightness enhancement. The brightness values given below therefore are only indicative of the typical intensities. Many of the airglow emissions arise in the ionospheric E layer at $\approx 90 \mathrm{~km}$, some in the $\mathrm{F}$ region above $150 \mathrm{~km}$ (see Fig. 18), some, like Ly $\alpha$ and $\mathrm{H} \alpha$ in the Geocorona. The phenomenological side of airglow, which is the part of interest for the night sky brightness, has for the vi- sual region in large part been studied in the sixties and seventies, which reflects in the list of references. Typical brightness values of main airglow lines are summarised in Table 13.

\subsection{Airglow spectrum}

a) Visual

Broadfoot \& Kendall (1968) give the spectrum of the airglow from $300 \mathrm{~nm}$ to $1 \mu \mathrm{m}$ (see Fig. 20). It is based on photoelectric observations at Kitt Peak near zenith and within $30^{\circ}$ of the galactic pole. The spectral resolution is $5 \AA$, the scan step four times smaller. The [OI] lines at $630 \mathrm{~nm}$ and $636.4 \mathrm{~nm}$ and also $\mathrm{H} \alpha$ are weaker than average in these observations.

\section{b) Ultraviolet}

Ultraviolet astronomical observations mostly are taken from above the atmosphere by rockets or satellites. In this 


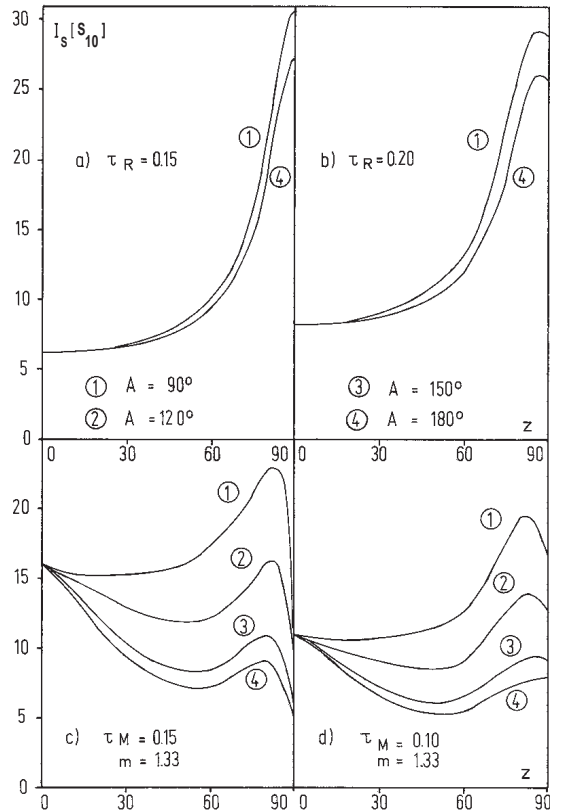

Fig. 15. The intensity of the scattered integrated starlight as a function of zenith distance, for different azimuths and zenith extincion values of the Rayleigh resp. Mie components of the atmosphere. The galactic centre is assumed at the zenith, the galactic equator crosses the horizon at $A=90^{\circ}, 270^{\circ}$

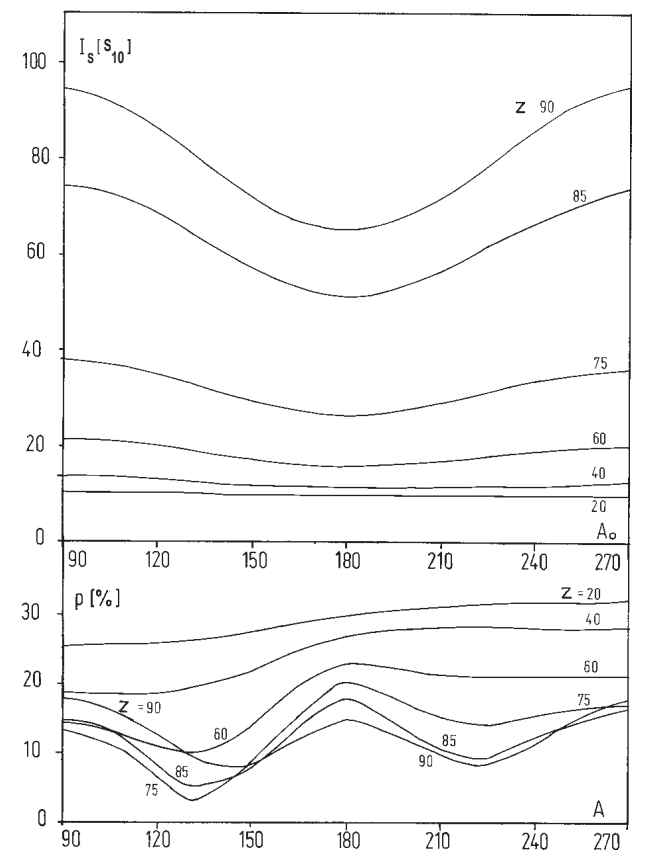

Fig. 16. The intensity of the scattered zodiacal light for a pure Rayleigh atmosphere with optical thickness $\tau_{\mathrm{R}}=0.1$. Position of the sun at azimuth $A_{\odot}=90^{\circ}$, zenith distance $z_{\odot}=105^{\circ}$, the ecliptic is perpendicular to the horizon

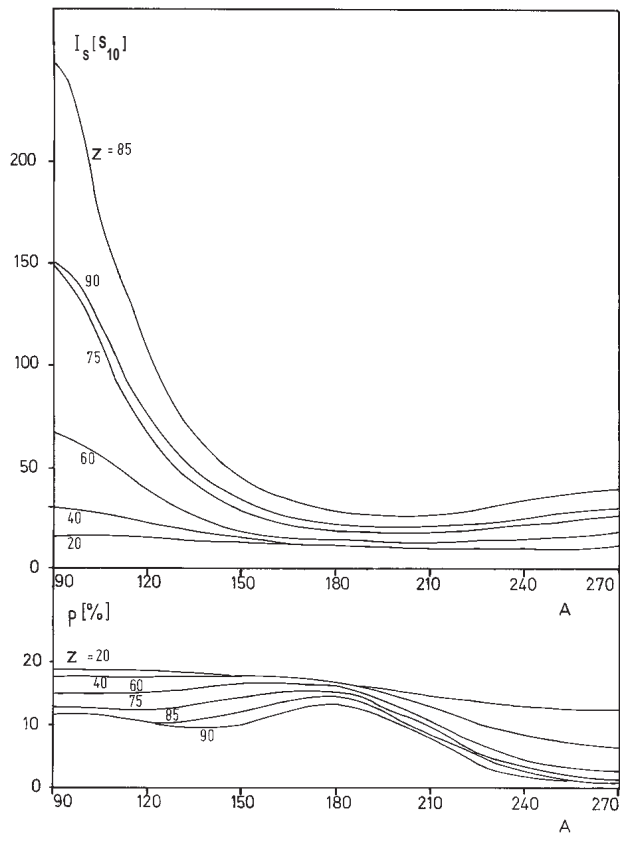

Fig. 17. The intensity of the scattered zodiacal light for pure Mie scattering with optical thickness $\tau_{\mathrm{M}}=0.1$, for particles with refractive index $m=1.33$. Otherwise same as for Fig. 16

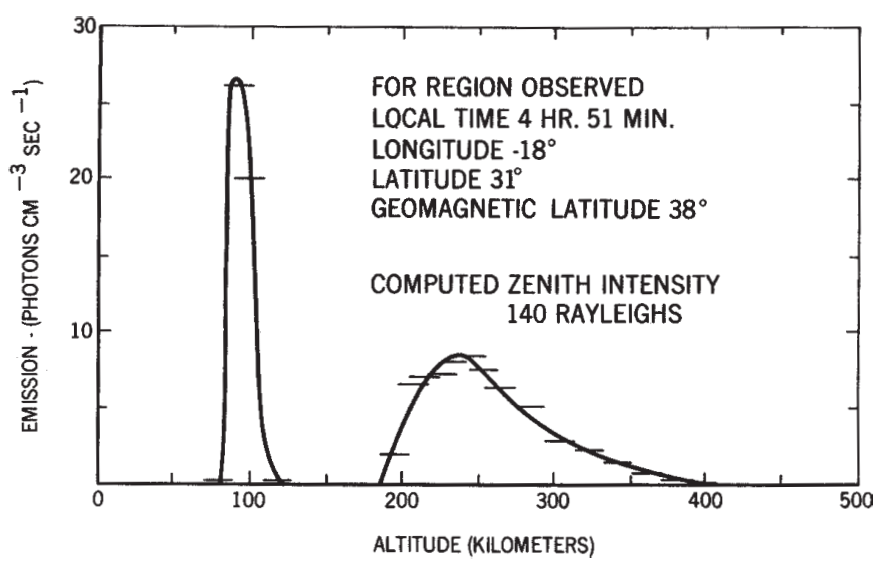

Fig. 18. A typical height profile of airglow volume emission, as measured from the satellite OGO II. The peak near $90 \mathrm{~km}$ is due to $\mathrm{OH}$ emission, the extended peak at higher altitudes to [OI] emission at $630 \mathrm{~nm}$. From Reed \& Blamont (1967) 


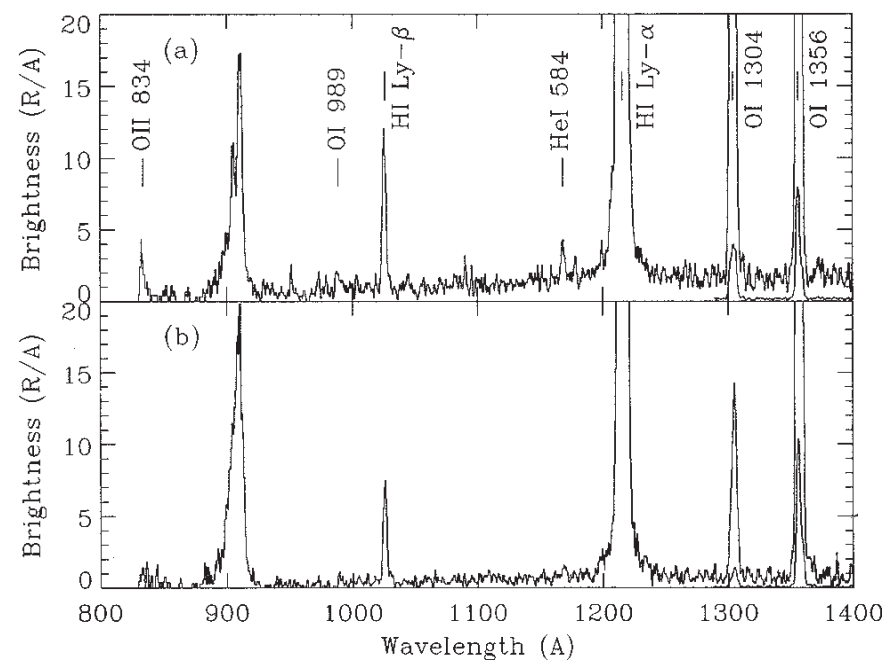

Fig. 19. Spectra of the nightglow from $800 \AA$ to $1400 \AA$ at $3 \AA$ resolution. The data were obtained from the space shuttle at an altitude of $358 \mathrm{~km}$ on December 5, 1990. Two spectra are shown, of which the upper one was taken closer to the dusk terminator. It therefore also shows OII 834 and HeI 584 (in second order), which are features belonging to the dayglow. The zenith distance was $\approx 85^{\circ}$ and $\approx 90^{\circ}$ for the upper and lower spectrum, respectively. Ly $\alpha$ is a geocoronal line. The continuum at $911 \AA$ is due to $\mathrm{O}^{+}$recombination to the ground state. From Feldman et al. (1992)

context it is relevant to know the airglow as seen from such spacecraft positions. Results obtained at typical altitudes are shown in Figs. 19 and 21. The strength of the main emission lines is also summarised in Table 13. For the OI $130.4 \mathrm{~nm}$ and $135.6 \mathrm{~nm}$ lines enhanced values observed in the tropical airglow (Barth \& Schaffner 1976) are given. At mid latitudes they are less intense by about one order of magnitude. Apart from the main emission lines shown in Fig. 19, the ultraviolet region between $850 \AA$ and $1400 \AA$ is thought to be free of nightglow emission.

The viewing line of spacecraft on the night side of the atmosphere may cross the terminator and continue through the sunlit parts of the atmosphere. Under these twilight conditions, dayglow features become important. E.g. the NO $\gamma$ bands then are excited by resonance fluorescence and then are much stronger, the $\mathrm{N}_{2}$ Lyman-BirgeHopfield bands are clearly visible, and the forbidden [OII] emission at $247 \mathrm{~nm}$ is strong. Figure 22 shows ultraviolet airglow emission observed under such conditions. An excellent review on observations and modelling of both dayglow and nightglow ultraviolet emissions has been given by Meier (1991).

\section{c) Near infrared}

From $1 \mu \mathrm{m}$ to $3 \mu \mathrm{m}, \mathrm{OH}$ in a layer around $90 \mathrm{~km}$ height dominates the airglow emission. There is a gap in the $\mathrm{OH}$ spectrum around $2.4 \mu \mathrm{m}$ (see Fig. 27) which is important for balloon observations and also for the low background observations possible from Antarctica (see Sect. 4.3). Seen from the ground, longward of $2.5 \mu$ airglow is only a small addition to the thermal emission from the troposphere (compare Fig. 11 in Sect. 4 above). Figures 25 and 26 show the near-infrared $\mathrm{OH}$ spectrum at two resolutions, once with a low spectral resolution of $\Delta \lambda=160 \AA$, and once with a higher resolution of $\lambda / \Delta \lambda=250-800$. Wavelength lists and intensities for the individual $\mathrm{OH}$ bands can be found in Ramsay et al. (1992) and Oliva \& Origlia (1992). Obviously, the near-infrared airglow is dominated by the $\mathrm{OH}$ bands. They primarily also determine the night sky brightness in the $J(1.2 \mu \mathrm{m})$ and $H(1.6 \mu \mathrm{m})$ bands (Fig. 11, Sect. 4.3).

\subsection{Dependence on zenith distance}

In absence of atmospheric extinction, a thin homogeneously emitting layer at height $h$ above the Earth's surface shows a brightness increase towards the horizon, which is given by the so-called van Rhijn function

$$
I(z) / I(\text { zenith })=\frac{1}{\sqrt{1-[R /(R+h)]^{2} \sin ^{2} z}},
$$

where $R=6378 \mathrm{~km}$ is the radius of the earth. E.g., for $h=100 \mathrm{~km}[I(z) / I(0)]_{\max }=5.7$ results (Roach \& Meinel 1955). This situation typically applies for balloon experiments. Figure 23 shows an example. For observations from the ground, extinction and scattering change the behaviour in particular for zenith distances $>40^{\circ}$. Around $\lambda=500 \mathrm{~nm}-600 \mathrm{~nm}$ a maximum airglow increase by about a factor of about four may be expected at $z=75^{\circ}-80^{\circ}$, with the brightness decreasing again towards the horizon (see Fig. 24 for an observation and Roach \& Meinel (1955) for a selection of predicted profiles). For shorter wavelengths, with stronger scattering and extinction, this decrease starts already at higher elevations. However, appropriate models (based on realistic assumptions, including multiple scattering in a spherical atmosphere and going down to the horizon) to account for the observed brightness profiles from the zenith to the horizon have not yet been calculated. The results given in Sect. 5 do not claim to be accurate near the horizon.

\subsection{Variations}

Airglow emission is often patchy and varying in brightness and spatial distribution with time. Roach \& Gordon (1973) demonstrate this by showing airglow maps in time steps of 15 minutes on the right upper corner of odd pages, 

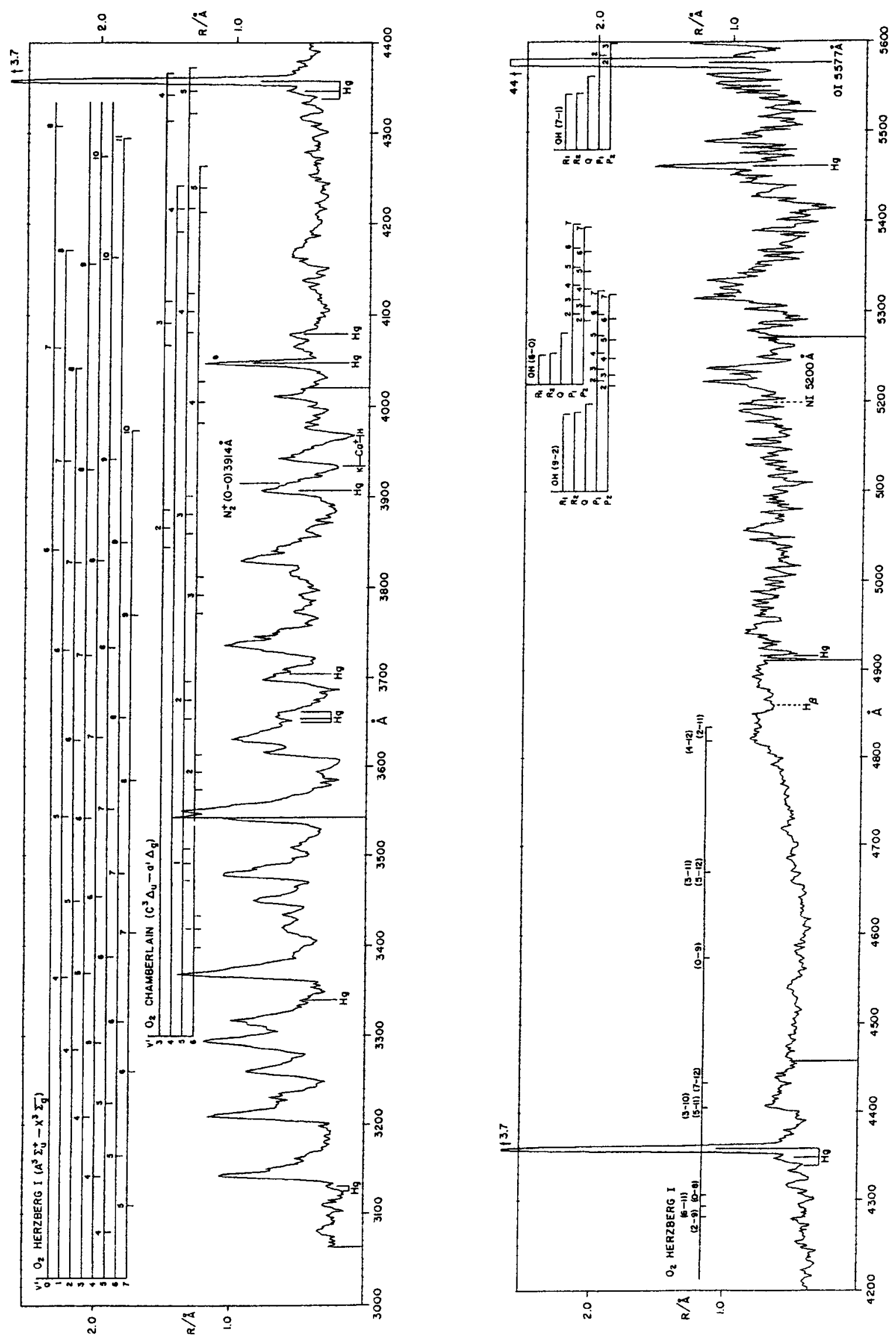

Fig. 20. Spectrum of the airglow from $300 \mathrm{~nm}$ to $1 \mu \mathrm{m}$ (from Broadfoot \& Kendall 1968) 

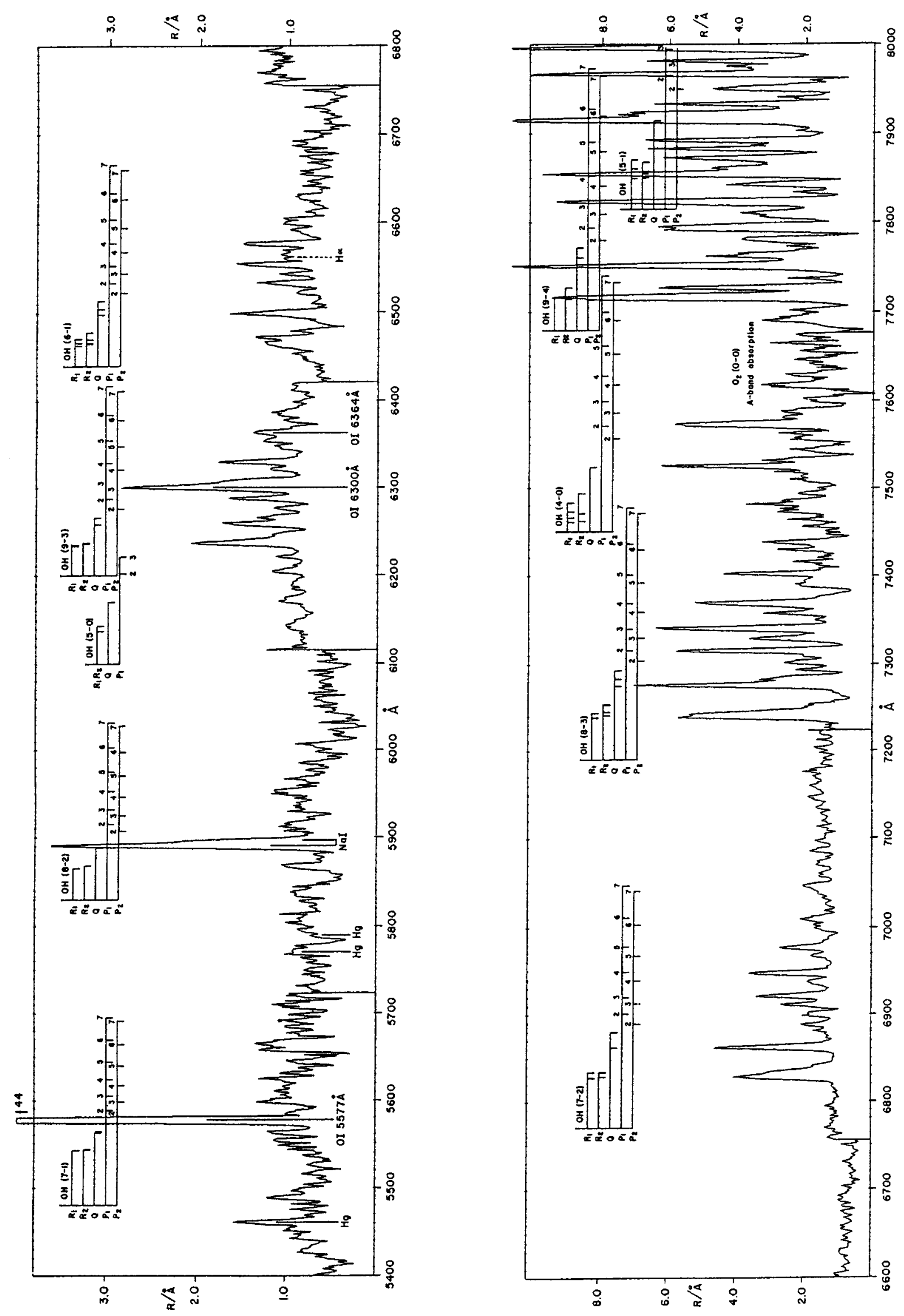

Fig. 20. continued 

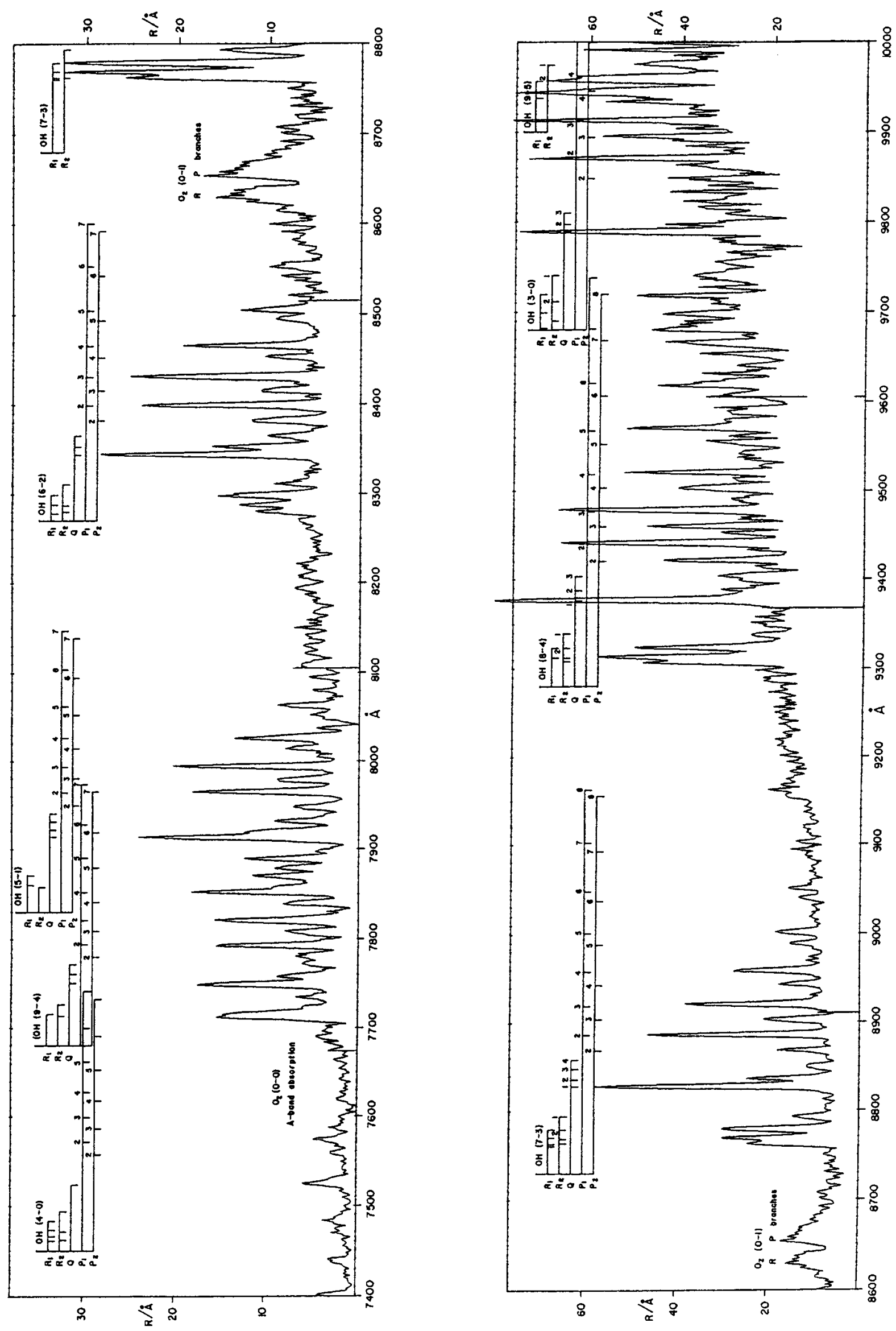

Fig. 20. continued 
Table 13. Typical zenith brightness of nightglow emissions ${ }^{a}$

\begin{tabular}{llcr}
\hline Source & Wavelength & $\begin{array}{c}\text { Height of } \\
\text { emitting layer }\end{array}$ & Intensity $^{b}$ \\
\hline $\mathrm{Ly} \beta$ & $102.6 \mathrm{~nm}$ & geocorona & geocorona \\
$\mathrm{Ly} \alpha$ & $121.6 \mathrm{~nm}$ & $250-300 \mathrm{~km}$ & $\approx 40 \mathrm{kR}$ (night) $-34 \mathrm{kR}($ day) \\
$\mathrm{OI}$ & $130.4 \mathrm{~nm}$ & $250-300 \mathrm{~km}$ & $\approx 30 \mathrm{R}$ (in tropical airglow) \\
$\mathrm{OI}$ & $135.6 \mathrm{~nm}$ & $90 \mathrm{~km}$ & $0.8 \mathrm{R} / \AA$ \\
$\mathrm{O}_{2}$ (Herzberg bands) & $300 \mathrm{~nm}-400 \mathrm{~nm}$ & $90 \mathrm{~km}$ & $250 \mathrm{R}$ \\
{$[\mathrm{OI}]$} & $557.7 \mathrm{~nm}$ & $\approx 92 \mathrm{~km}$ & $30 \mathrm{R}$ (summer) \\
$\mathrm{NaD}$ & $589.0 \mathrm{~nm}, 589.6 \mathrm{~nm}$ & & to $100 \mathrm{R}$ (winter) \\
& & $250-300 \mathrm{~km}$ & $60 \mathrm{R}$ \\
$\mathrm{OI}]$ & $630.0 \mathrm{~nm}$ & $250-300 \mathrm{~km}$ & $20 \mathrm{R}$ \\
{$[\mathrm{OI}]$} & $636.4 \mathrm{~nm}$ & geocorona & $4-6 \mathrm{R}$ (night) \\
$\mathrm{H} \alpha$ & $656.3 \mathrm{~nm}$ & $90 \mathrm{~km}$ & $0.3 \mathrm{R} / \AA$ \\
$\mathrm{pseudocontinuum}$ & $400 \mathrm{~nm}-700 \mathrm{~nm}$ & $\approx 80 \mathrm{~km}$ & $1 \mathrm{kR}$ \\
$\mathrm{O}_{2}$ & $864.5 \mathrm{~nm}$ & $85 \mathrm{~km}$ & $4.5 \mathrm{MR}($ all bands) \\
$\mathrm{OH}$ & $600 \mathrm{~nm}-4.5 \mu \mathrm{m}$ & &
\end{tabular}

${ }^{a}$ after Chamberlain (1961), Roach (1964), Roach \& Gordon (1973), Meier (1991); see also the references in the sections on geocorona and ultraviolet airglow.

${ }^{b}$ transformed to zenith, where necessary.
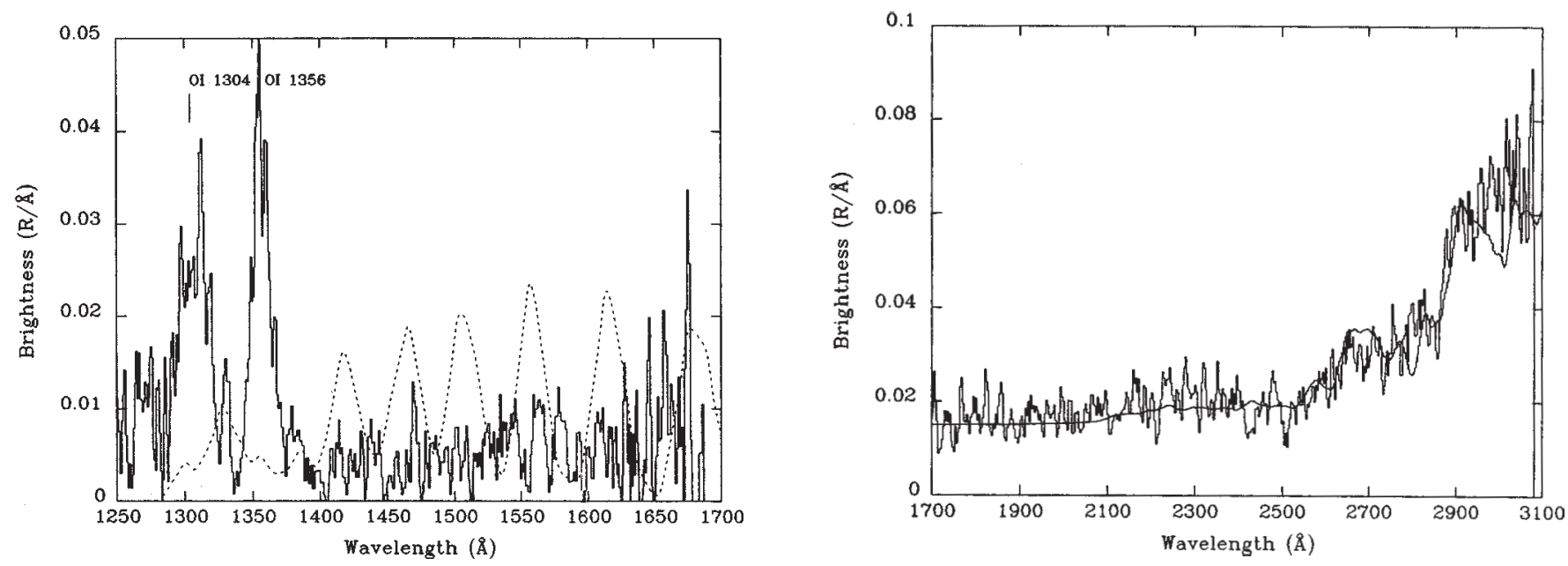

Fig. 21. Left: Spectrum of the nightglow from $1250 \AA$ to $1700 \AA$ at $17 \AA$ resolution. The data were obtained from the space shuttle at a height of $330 \mathrm{~km}$ in January 1986 at minimum solar activity. The oxygen OI lines at $1304 \AA$ and $1356 \AA$ are the brightest features. For the weakly visible Lyman-Birge-Hopfield bands the dashed curve shows a predicted spectrum. Right: Spectrum of the ultraviolet nightglow from $170 \mathrm{~nm}$ to $310 \mathrm{~nm}$ at $29 \AA$ resolution obtained on the same flight. The solid line shows an appropriately scaled solar spectrum and is assumed to show the contribution to zodiacal light. From Morrison et al. (1992)

thus enabling a "thumb-cinema" look at these spatiotemporal variations. Quantitative examples for variation during one night or variation with solar cycle can be seen in Figs. 8 and 10 in Sect. 4. Often a systematic decrease of airglow emission during the course of the night is observed, explained as result of the energy stored during day in the respective atmospheric layers.

Figure 29 shows this for the $\mathrm{OH}$ emissions and also gives an example for the wavelike structures often apparent in these emissions.
These examples do not give at all a full overview on airglow variability but just demonstrate that it is a typical property of this source of night sky brightness.

In the visual spectral region, correlations between the prominent [OI] and $\mathrm{NaD}$ airglow emission lines and "pseudocontinuum" bands at $367 \mathrm{~nm}, 440 \mathrm{~nm}, 526 \mathrm{~nm}, 558 \mathrm{~nm}$, $634 \mathrm{~nm}$ and $670 \mathrm{~nm}$ have been studied by Barbier (1956) who established three "covariance groups". E.g., the correlation between the $557.7 \mathrm{~nm}$ line and the "pseudocontinuum" at $502 \mathrm{~nm}$ has been used by Dumont (1965) to 

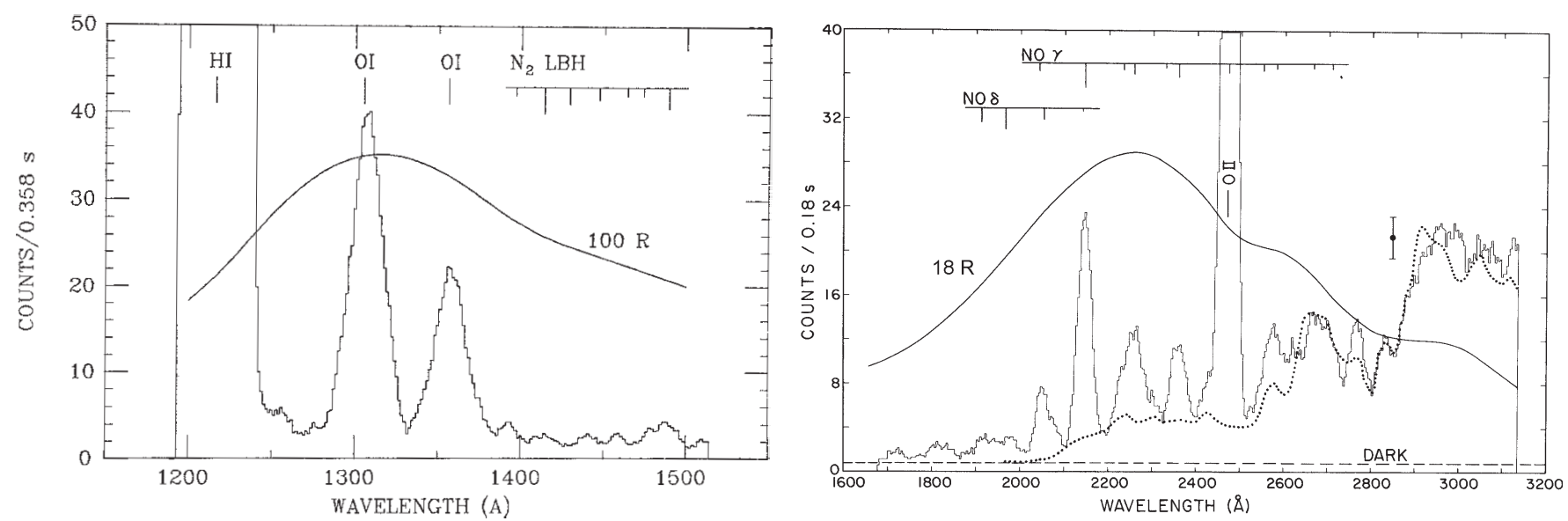

Fig. 22. Ultraviolet twilight airglow spectrum, as observed during a rocket flight on September 24, 1979. Left: from $1200 \AA$ to $1500 \AA$ at $20 \AA$ resolution. Ly $\alpha$ is at left. "LBH" refers to the Lyman-Birge-Hopfield bands. These observations were done in the height range $100 \mathrm{~km}-200 \mathrm{~km}$. - Right: From $170 \mathrm{~nm}$ to $310 \mathrm{~nm}$ at $25 \AA$ resolution. The dotted line shows the zodiacal light contribution. These observations refer to rocket heights of $170 \mathrm{~km}-246 \mathrm{~km}$. - The field of view of the experiment was oriented $23^{\circ}$ from the sun and essentially in the horizontal plane $\left(0.2^{\circ}\right.$ elevation $)$. For conversion to absolute fluxes, a solid line is given with both parts of the figure. It indicates which signal would be produced at each wavelength by a monochromatic source of a given brightness (100 R for the short-wavelength part, $18 \mathrm{R}$ for the longer wavelengths). For continuum emission this would correspond to $5.0 \mathrm{R} / \AA$ and $0.72 \mathrm{R} / \AA$, respectively. From Cebula \& Feldman $(1982,1984)$

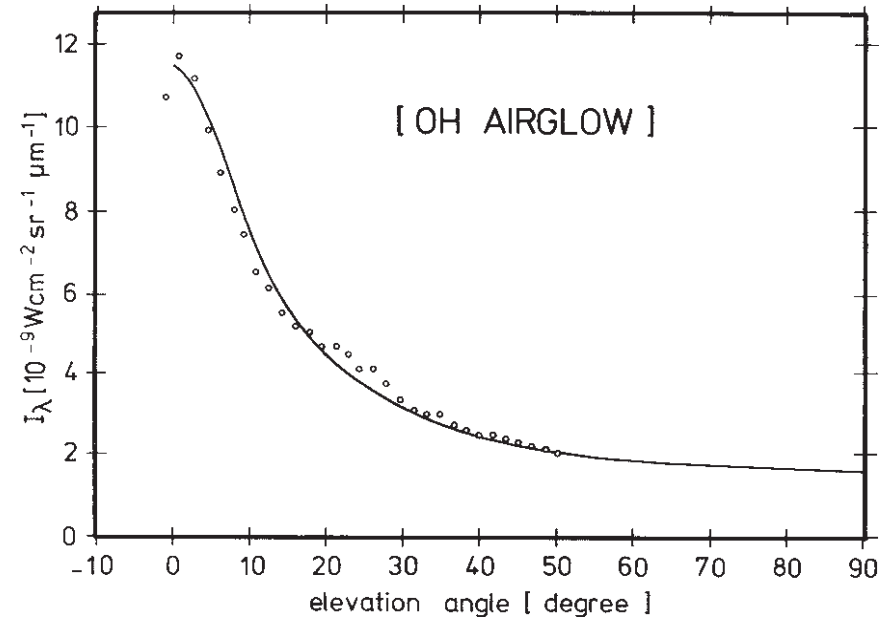

Fig. 23. Increase of airglow brightness at $2.1 \mu \mathrm{m}$ towards the horizon observed from a balloon at $30 \mathrm{~km}$ altitude on October 23, 1972. Dots represent the measurements, the line gives the van Rhijn function for a height of the emitting layer of $92 \mathrm{~km}$. From Hofmann et al. (1977)

eliminate the airglow contribution from his zodiacal light measurements. Sometimes such correlations can be quite tight (see Fig. 28).

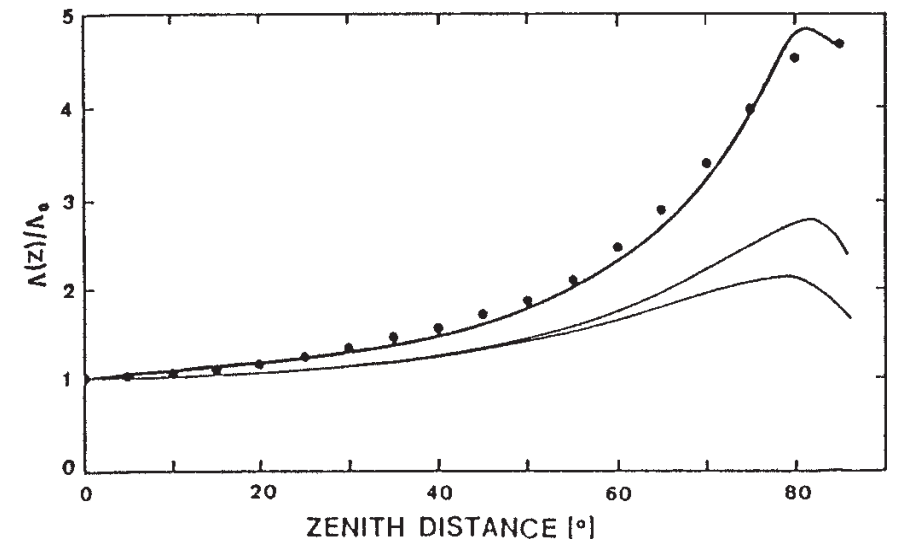

Fig. 24. Zenith angle dependence of sky brightness observed at $530 \mathrm{~nm}$ from Mt. Haleakala, Hawaii (Kwon et al. 1991). The points represent an average normalised profile. The thin lines are the curves predicted by Barbier in 1944 for heights of the airglow emitting layer of $50 \mathrm{~km}$ (higher maximum) and $200 \mathrm{~km}$, respectively. The solid line fitting the data is an ad-hoc modification of Barbier's formula

\subsection{Geocorona}

Above $1000 \mathrm{~km}$, the earths atmosphere changes to a composition of mainly neutral hydrogen with some ionised helium, the density falling off gradually over a few earth 

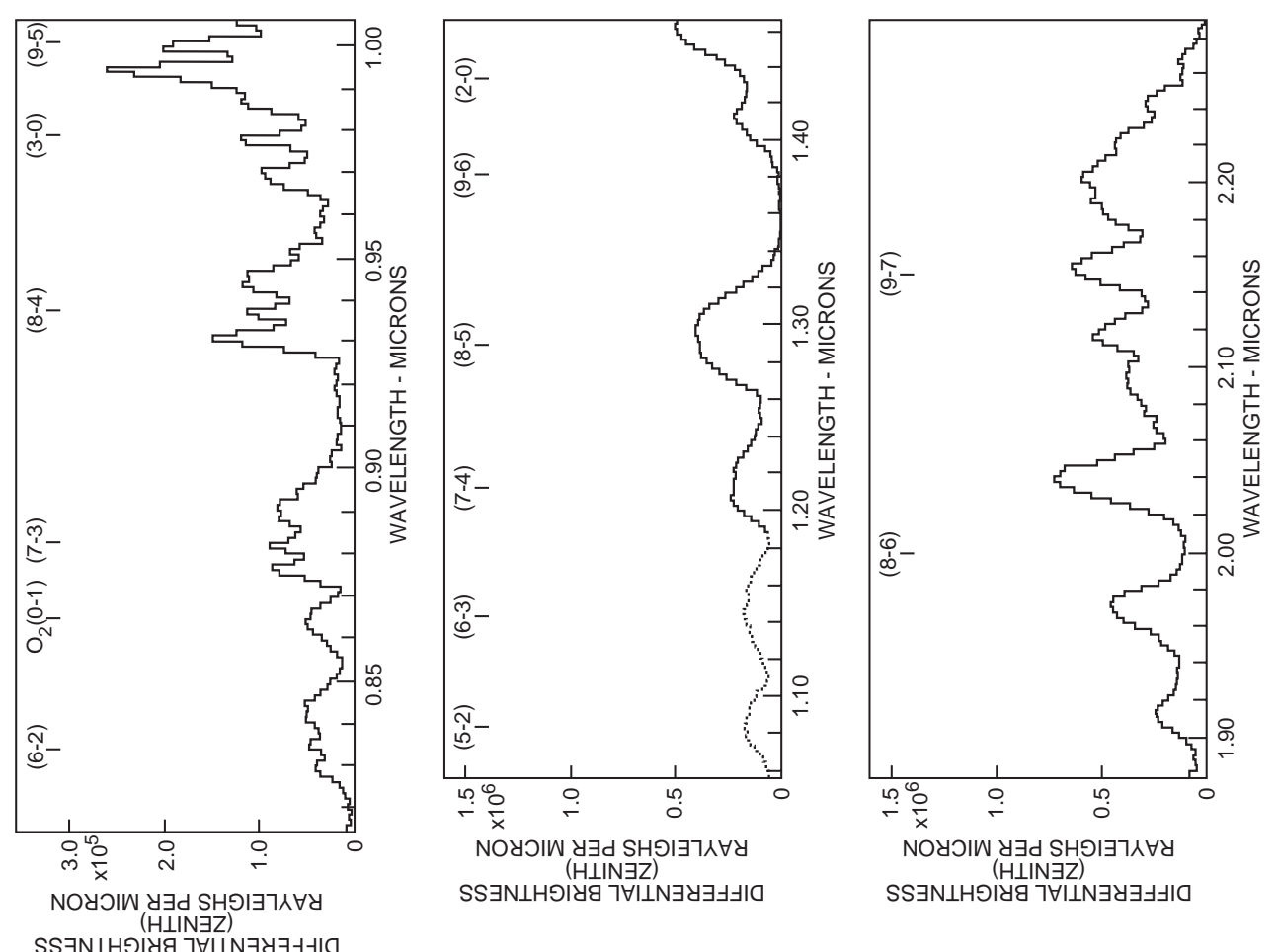

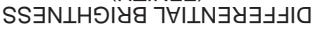
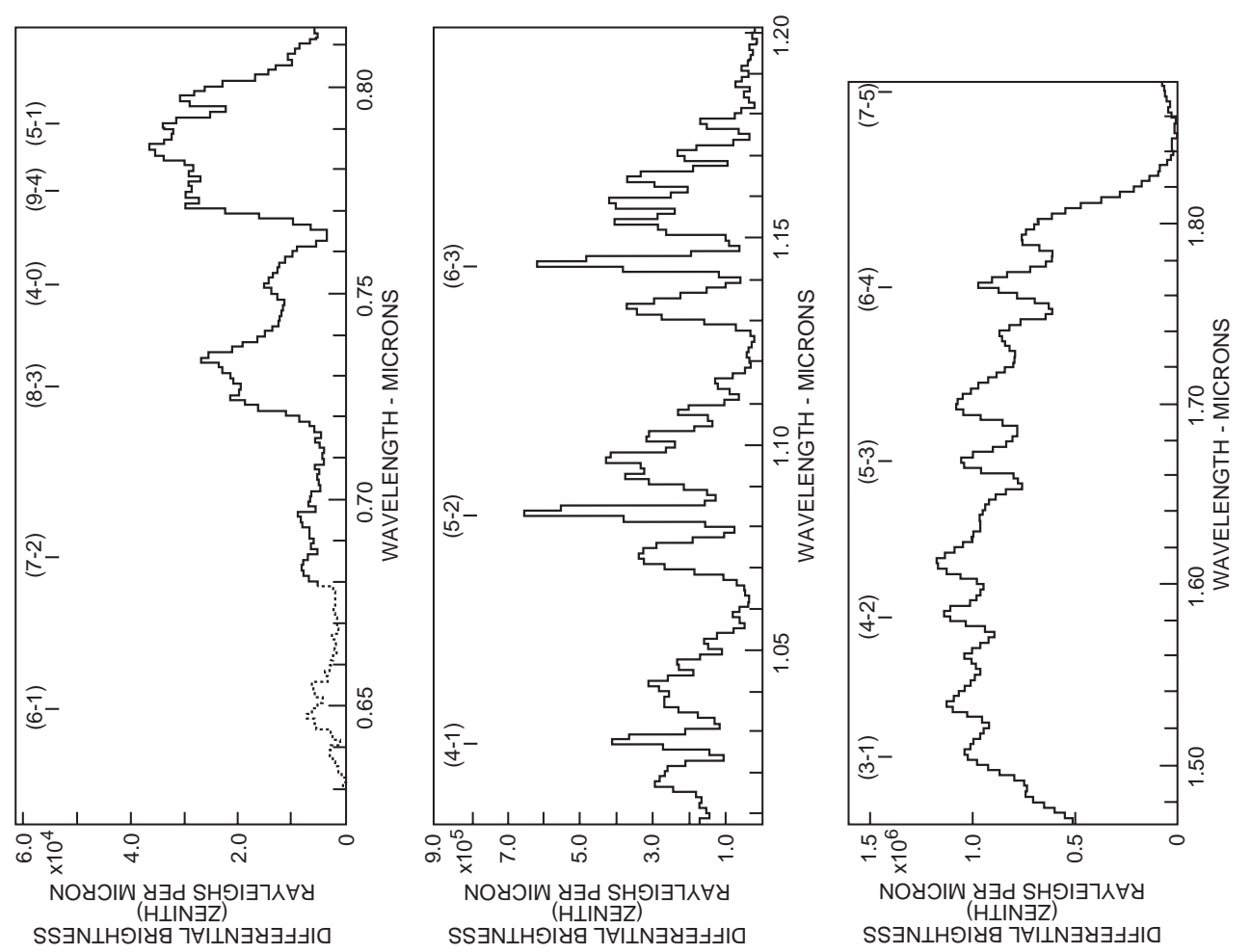

Fig. 25. Near-infrared airglow spectrum as seen from the ground at $160 \AA$ resolution (for $\lambda>1.2 \mu \mathrm{m}$ ). The OH bands mainly contributing to the emission have been identified in the figure. "differential" simply means "per micron". From Harrison \& Kendall (1973) 

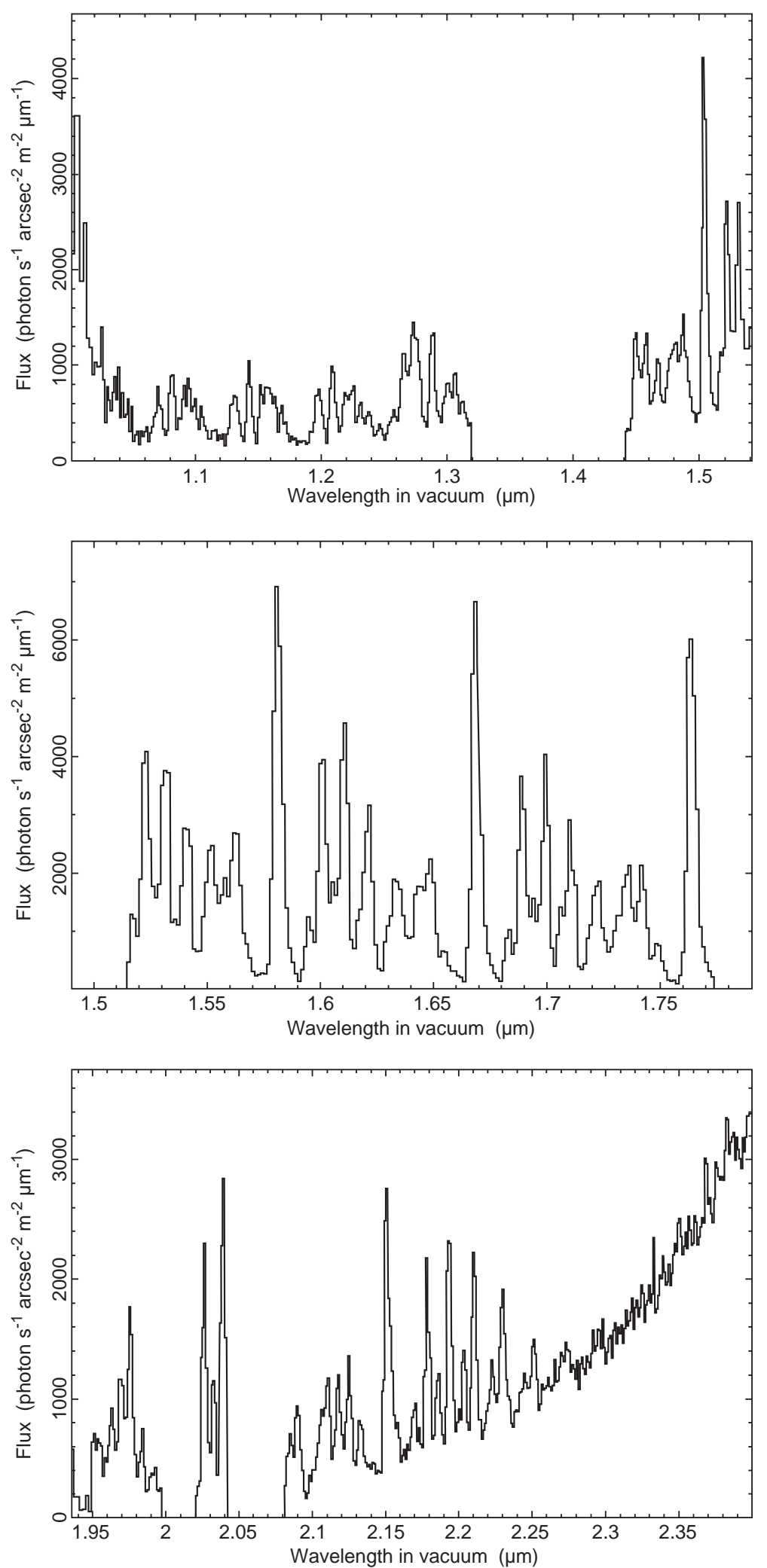

Fig. 26. Near-infrared airglow spectrum as observed from Mauna Kea at spectral resolution $\lambda / \Delta \lambda=250-800$. In regions with atmospheric transmission $\leq 0.75$ the flux has been arbitrarily set to zero. Longward of $2.1 \mu \mathrm{m}$ thermal atmospheric emission takes over. Note that 1000 of the units used correspond to $6.7710^{-6}, 5.1110^{-6}$, and $3.8410^{-6} \mathrm{~W} / \mathrm{m}^{2} \mathrm{sr} \mu \mathrm{m}$ at $1.25 \mu \mathrm{m}, 1.65 \mu \mathrm{m}$ and $2.2 \mu \mathrm{m}$, respectively. From Ramsay et al. (1992) 

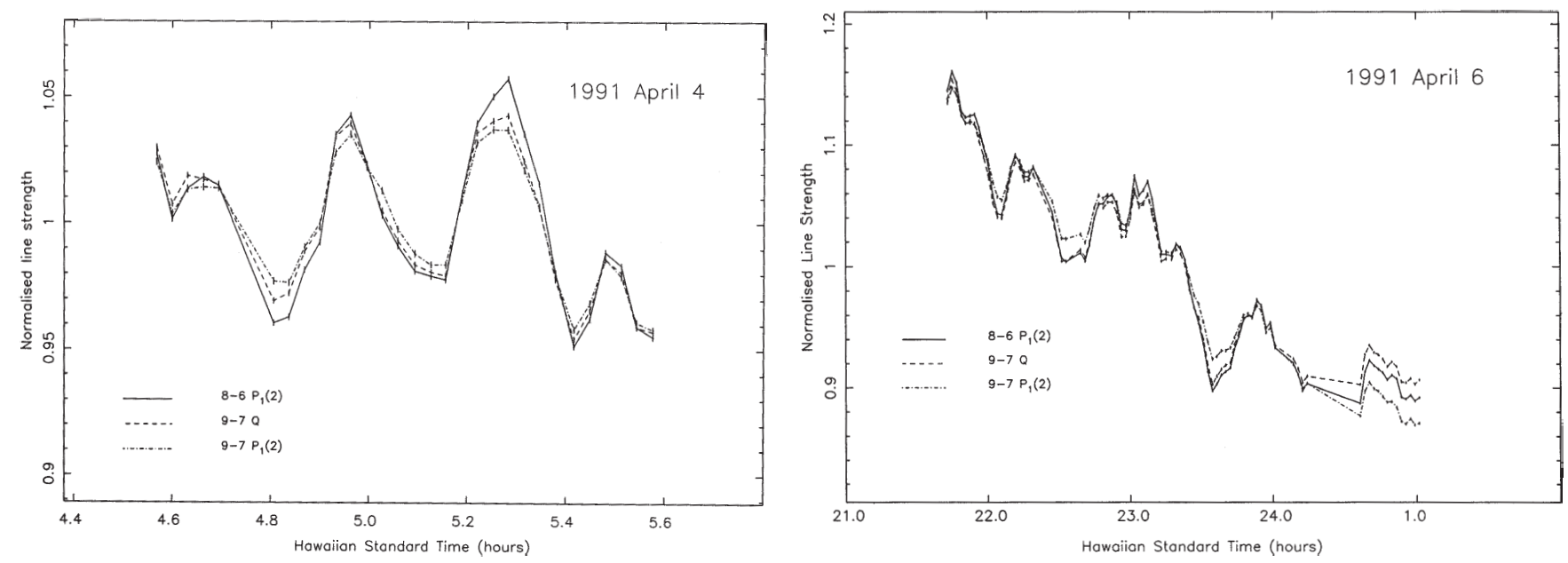

Fig. 29. Variation of $\mathrm{OH}$ airglow, observed from Mauna Kea. Left: Short term variations (minutes) caused by the passage of wavelike structures. Right: Decrease of $\mathrm{OH}$ airglow during the course of a night, shown for several bands separately. From Ramsay et al. (1992)

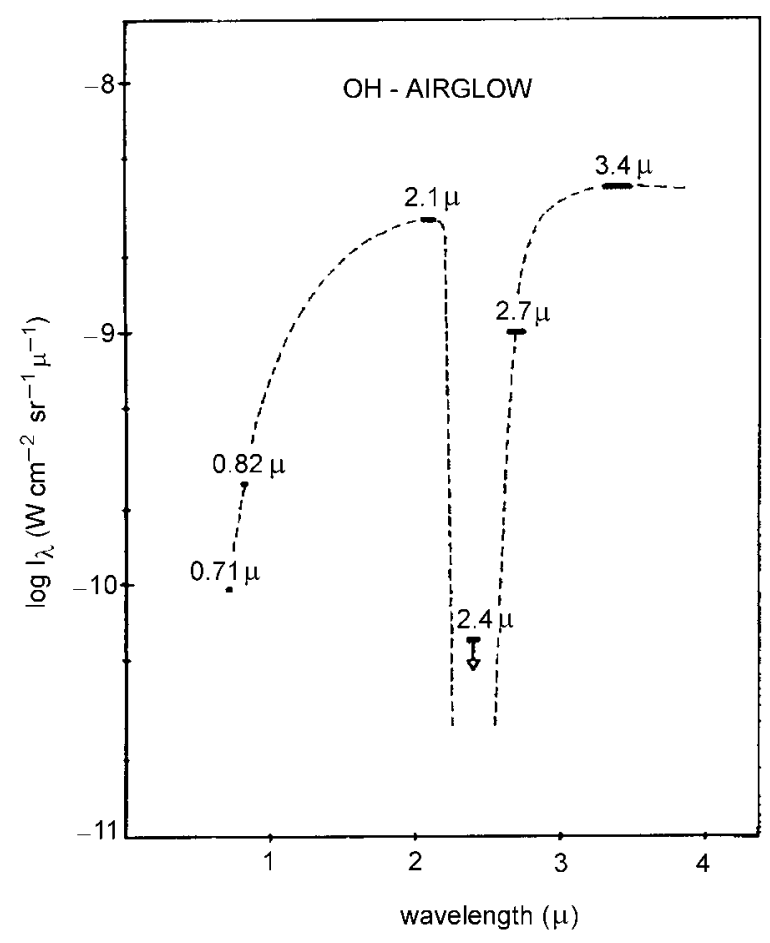

Fig. 27. Spectral distribution of near-infrared zenith airglow showing the gap in airglow emission around $2.4 \mu \mathrm{m}$. The airglow measurements have been performed from a balloon at $30 \mathrm{~km}$ altitude during flights in 1972 and 1974 . Variations from flight to flight and during one night were less than a factor of two. From Hofmann et al. (1977)

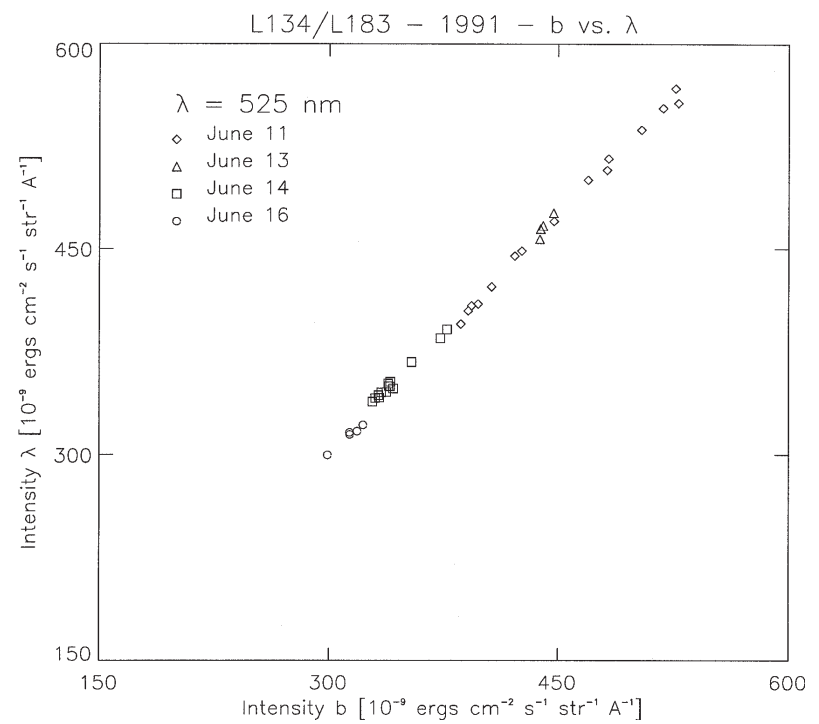

Fig. 28. Correlation between the diffuse sky emission at 467 $\mathrm{nm}$ (Strömgren $b$ ) and at $\lambda=525 \mathrm{~nm}$. The brightness variations in both bands are mainly due to airglow. From Leinert et al. (1995)

radii. Two telling images of the geocorona in Ly $\alpha$, including the globe of the earth, are shown by Frank et al. (1985, see p. 63). This geocorona is optically thick to the solar Lyman lines. Typical intensities of the emissions observed from ground (in the visual) or from earth orbit are given in Table 13, with the data taken from Caulet et al. (1994) and Raurden et al. (1986) for Ly $\alpha$, Meier et al. (1977) for $\mathrm{Ly} \beta$, Levasseur et al. (1976) for $\mathrm{H} \alpha$. 

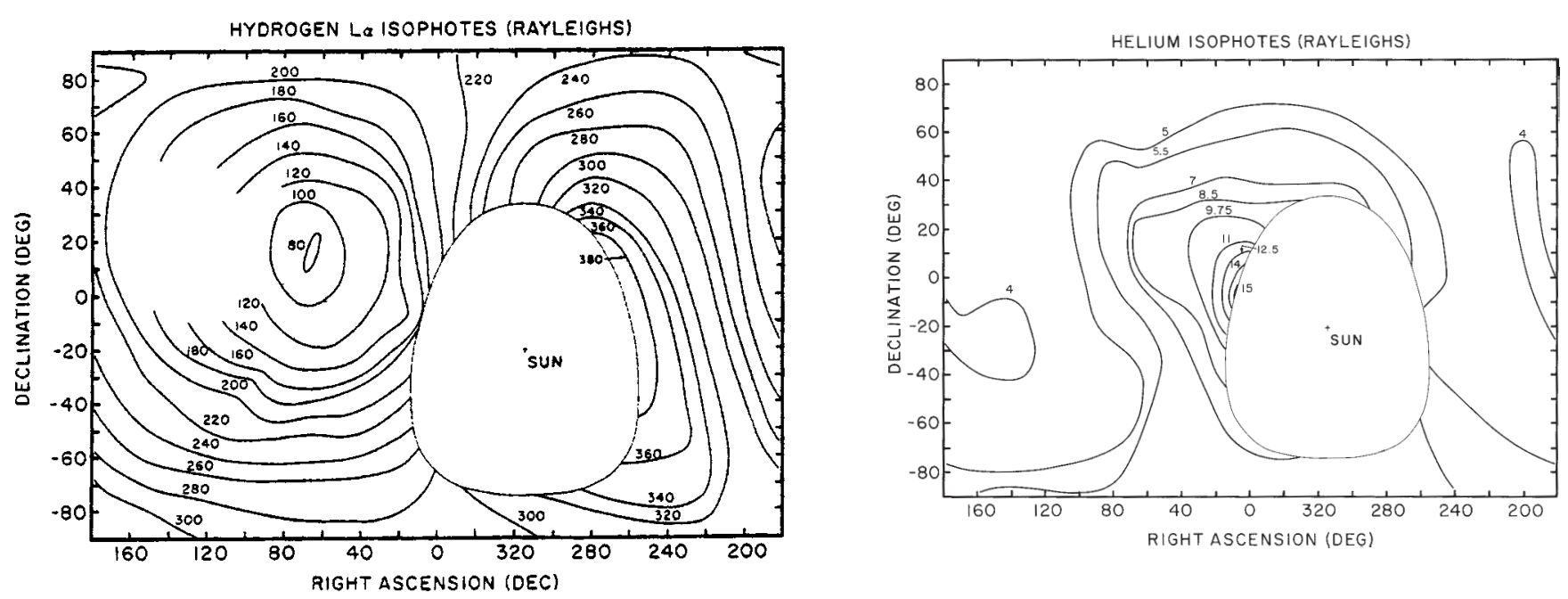

Fig. 30. Interplanetary emission in the Ly $\alpha$ (left) and He $584 \AA$ lines (right) observed by the Mariner 10 UV spectrometer (Broadfoot \& Kumar 1978). The observations were performed on January 28, 1974, while the spacecraft was at a heliocentric distance of $0.76 \mathrm{AU}$ and $\approx 60^{\circ}$ from the apex-Sun axis. The brightness units are Rayleighs. From Thomas (1978)

\subsection{Interplanetary emissions}

Solar radiation is scattered by neutral interstellar gas atoms which are coming from the solar apex direction and are pervading the solar system until ionized. The emitting region is a sort of cone around the apex-Sun line. The observed emission depends on the position of a spacecraft with respect to this cone (see, e.g. the review by Thomas 1978). Typical patterns observed for the Ly $\alpha$ and He $584 \AA$ lines are shown in Fig. 30.

\subsection{Shuttle glow}

Depending on altitude and solar activity, satellites produce additional light emissions by interaction with the upper atmosphere (Shuttle glow). Photometric measurements thus may be affected. These light phenomena are relatively strong in the red and near-infrared spectral regions, but are noticeable towards the ultraviolet as well.

For instance, during the Spacelab 1 mission the emissions of the $\mathrm{N}_{2}$ Lyman-Birge-Hopfield bands were found to be in the range of $10-50 \mathrm{R} / \AA$ (Torr et al. 1985). These observations at $250 \mathrm{~km}$ altitude were performed under conditions of moderate solar activity. During minimum solar activity and at $330 \mathrm{~km}$, Morrison et al. (1992) observed no such emissions. The GAUSS camera onboard the German Spacelab mission D2 (296 km, moderate solar activity) observed a patchy glow with $\approx 0-310^{-9} \mathrm{~W} \mathrm{~m}^{-2} \mathrm{sr}^{-1} \mathrm{~nm}^{-1}$ at $210 \mathrm{~nm}$ (Schmidtobreick 1997). Taking into account the appropriate conversion factor, the observed glow intensity amounts to about $0.4 \mathrm{R} / \AA$ in its brightest parts. Although these three observations were made at somewhat different wavelengths, the overall increase of emission intensity $I$ with surrounding air density $\rho$ is in agreement with an $I \sim \rho^{3}$ law.

Table 14. The strongest artficial emission lines in the night sky spectrum between $3600-8200 \AA$. The most intense features are shown in boldface

\begin{tabular}{|l|l|}
\hline Line & Sources \\
\hline Hg I 3650 & Hg lighting \\
Hg I 3663 & Hg lighting \\
Hg I 4047 & Hg lighting \\
Hg I 4078 & Hg lighting \\
Hg I 4358 & Hg lighting \\
Na I 4665, 4669 & HPS \\
Na I 4748, 4752 & HPS \\
Na I 4978, 4983 & HPS \\
Na I 5149, 5153 & HPS \\
Hg I 5461 & Hg lighting \\
Na I 5683, 5688 & HPS, LPS \\
Hg I 5770 & Hg lighting \\
Hg I 5791 & Hg lighting \\
Na I 5890, 5896 & HPS, LPS, airglow \\
Na I 5700- $\mathbf{6 1 0 0}$ & HPS \\
(wings) & \\
Na I 6154, $\mathbf{6 1 6 1}$ & HPS, LPS \\
K I 7665 & HPS, LPS \\
K I 7699 & HPS, LPS \\
Na I 8183 & HPS, LPS \\
Na I 8195 & HPS, LPS \\
\hline
\end{tabular}




\section{Light pollution}

Artificial lighting at earth contributes via tropospheric scattering to the night sky brightness over a large area around the source of light. Both a continuous component as well as distinct emission lines are present in the light pollution spectrum. A recent review of sky pollution is given in McNally (1994).

\subsection{Observations of sky pollution}

Systematic broad-band observations of the sky pollution light near cities have been carried out by Bertiau et al. (1973) in Italy, Berry (1976) in Canada \& Walker (1970, 1977 ) in California. Berry showed that there is a relationship between the population of a city and the zenith sky brightness as observed in or near to the city. Walker interpreted his extensive observations by deriving the following relationships: (1) between the population and luminosity of a city; (2) the sky brightness as a function of distance from the city; and (3) between the population and the distance from a city for a given sky pollution light contribution. The last two relationships are shown in Figs. 31 and 32. These figures can be utilized to derive an estimate for the sky pollution at $45 \mathrm{deg}$ altitude caused by a city with 2000-4 million population and with a similar street lighting power per head as California. Starting with the city population Fig. 31 gives the distance at which the artificial lighting contribution increases the natural sky brightness by $20 \%\left(0.2 \mathrm{mag} / \square^{\prime \prime}\right)$. With this distance one can enter Fig. 32 and obtain a scaling for the (arbitrary) intensity axis of this figure. Thus the artificially caused sky brightness at $45 \mathrm{deg}$ altitude at $6-200 \mathrm{~km}$ distance from the city can be estimated from this figure.

\subsection{Modelling of sky pollution}

Treanor (1973) and Bertiau et al. (1973) have used an empirical formula, based on a simplified model of the tropospheric scattering, to fit the sky pollution observations near cities. Garstang (1986, 1989a,b, 1991) has used radiative transfer models including 1st and 2nd order Rayleigh and aerosol scattering, effects of ground albedo and curvature of the earth's surface, and the areal distribution of the light source to calculate the sky pollution light intensity. He has compared and scaled his model results against the above mentioned observational results. Garstang's fitted models are shown in Figs. 31 and 32. superimposed on the observational points of Walker (1977). Garstang (1986, 1989a,b) gives also the calculated zenith distance dependence of the sky pollution light intensity both towards and away from the source of light. These results are reproduced in Fig. 33.

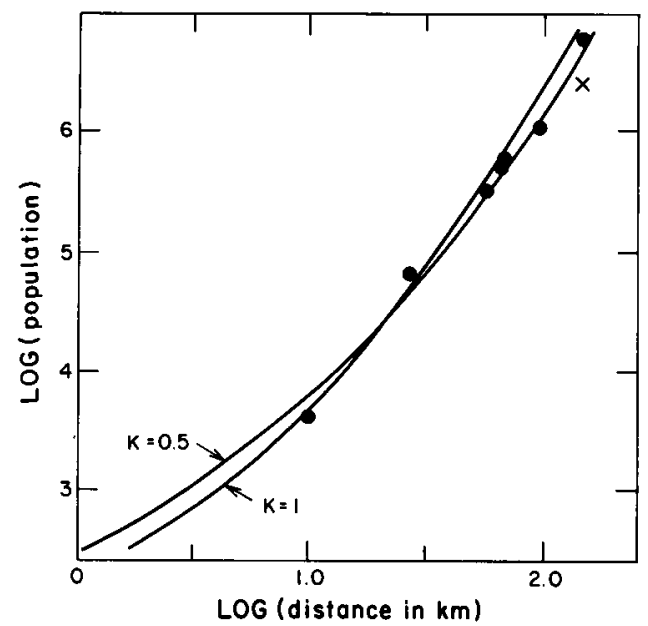

Fig. 31. Variation with city population of the distance at which the lights of a city produce an artificial increase of the night sky brightness at $45 \mathrm{deg}$ altitude toward the city by $0.20 \mathrm{mag}$. This increase refers to an assumed natural sky brightness of $V=21.9 \mathrm{mag} / \square^{\prime \prime}$. Observations by Walker (1977) are indicated by dots. Two models by Garstang (1986) are shown as solid lines. $K$ is a measure for the relative importance of aerosols for scattering light. The uppermost dot refers to Los Angeles County, the cross below it to Los Angeles City. From Garstang (1986)

\subsection{Spectrum of the sky pollution light}

The emission line spectra of the different types of street lamps are visible in the night sky light even at good observatory sites, such as Kitt Peak in Arizona. While the most commonly used street lamps until the 1970's were filled with $\mathrm{Hg}$ there has been since then a general change over to sodium lamps, both of the high pressure (HPS) and low pressure sodium (LPS) types. The most important sky pollution lines are given in Table 14 according to Osterbrock et al. (1976), Osterbrock \& Martel (1992) and Massey et al. (1990). At good sites (e.g. Kitt Peak), the strongest pollution lines are about a factor of two weaker than the strongest airglow lines. The opposite is true for strongly contaminated sites (e.g. Mt Hamilton). Whereas the pollution lines are normally restricted to a relatively narrow wavelenth range the $\mathrm{Na} \mathrm{D}$ line wings produced by the HPS lamps are extremely broad, extending over $5700-6100 \AA$. Thus the LPS lamps are highly preferable over the HPS ones from the astronomer's point of view.

Other studies of the night sky spectrum, including the artificial pollution lines, have been presented by Broadfoot \& Kendall (1968) for Kitt Peak, Turnrose (1974) for Mt. Palomar and Mt. Wilson, and Louistisserand et al. (1987) for Pic du Midi. 


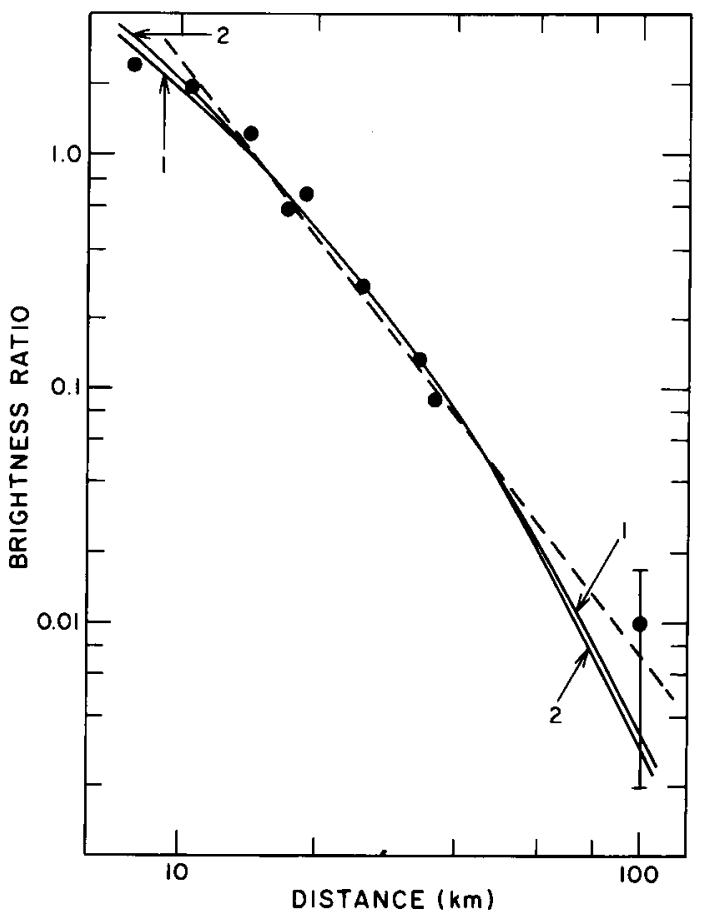

Fig. 32. Variation with distance from the city of the sky brightness at $45 \mathrm{deg}$ altitude in the direction of the city. The dots indicate observations in $V$ band by Walker (1977) near the city of Salinas. The solid curves are according to models by Garstang (1986). The brightness ratio is defined as $\frac{b\left(\text { Salinas at }+45^{\circ}\right)-b\left(\text { Salinas at }-45^{\circ}\right)}{b\left(\text { sky background only at }+45^{\circ}\right)}$, where $b=$ sky brightness. Zenith distance $+45^{\circ}$ is towards and $-45^{\circ}$ away from the city. The solid curves are according to models by Garstang (1986). Curve 1: $L_{0}=986$ lumens per head, $K=0.43, F=11 \%$. Curve 2: $L_{0}=1000$ lumens per head, $K=0.5, F=10 \% . L_{0}$ is the artificial lighting in lumens produced per head of the population. $K$ is a measure for the relative importance of aerosols for scattering light. $F$ : a fraction $F$ of the light produced by the city is radiated directly into the sky at angles above the horizontal plane, and the remainder $(1-F)$ is radiated toward the ground. The dashed line is the relation $\sim D^{-2.5}$. From Garstang (1986)

\section{Zodiacal light}

\subsection{Overview and general remarks}

The zodiacal light in the ultraviolet, visual and nearinfrared region is due to sunlight scattered by the interplanetary dust particles. In the mid- and far-infrared it is dominated by the thermal emission of those particles. Zodiacal light brightness is a function of viewing direction $\left(\lambda-\lambda_{\odot}, \beta\right)$, wavelength, heliocentric distance $(R)$ and position of the observer relative to the symmetry plane of interplanetary dust. Its brightness does not vary with

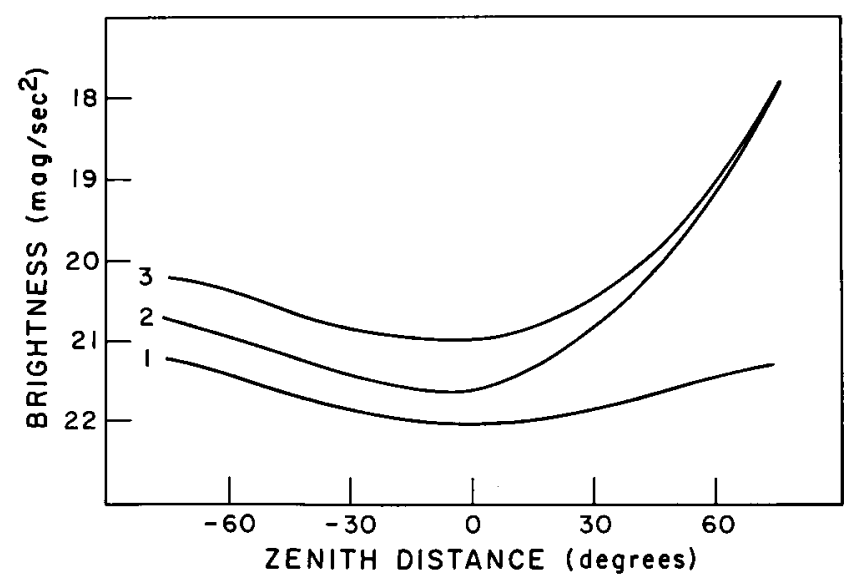

Fig. 33. Zenith distance dependence of sky pollution light according to the model calculations of Garstang (1986). Results are for sky pollution due to Denver as seen from a distance of $40 \mathrm{~km}$ in the vertical plane containing the observer and the center of Denver. Curve 1: sky background; Curve 2: Denver only; Curve 3: Denver and sky background. Negative zenith distances are away from Denver. From Garstang (1986)

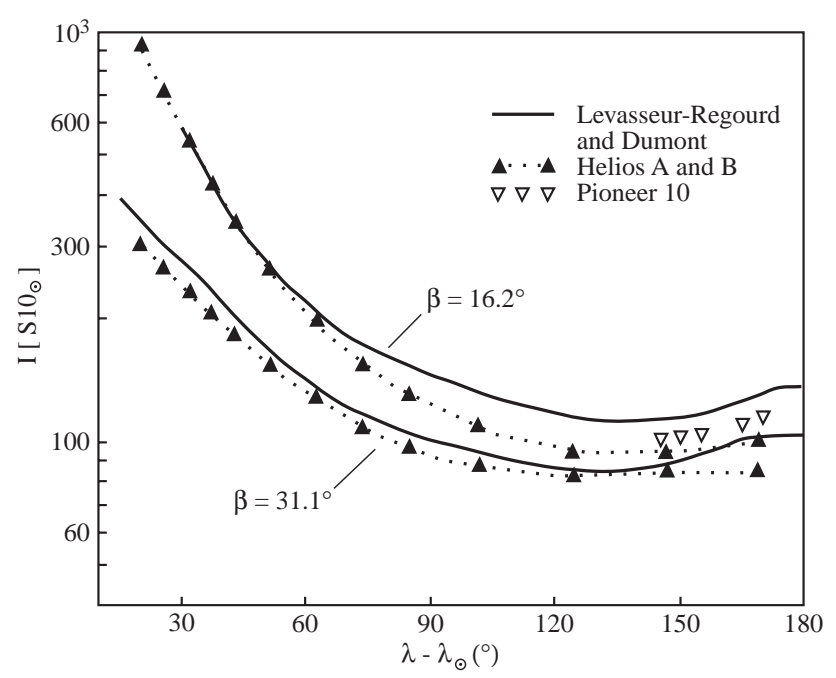

Fig. 34. Comparison of zodiacal light measurements along the bands of constant ecliptic latitude $\beta=16.2^{\circ}$ and $\beta=31.0^{\circ}$ observed by Helios A and B. The ground-based measurements of Levasseur-Regourd \& Dumont (1980) at $\lambda_{\text {eff }}=502 \mathrm{~nm}$ have been linearly interpolated to these latitude values. The Helios measurements at $B$ and $V$ (Leinert et al. 1981) have been linearly interpolated to $\lambda_{\text {eff }}=502 \mathrm{~nm}$. The Pioneer measurements (Toller \& Weinberg 1985) have been extrapolated from blue to $502 \mathrm{~nm}$ with the values applicable for Helios and from $\beta=$ $10^{\circ}$ to $\beta=16^{\circ}$ according to the table of Levasseur-Regourd \& Dumont (1980). For definition of the $S 10_{\odot}$ unit see Sect. 2 
solar cycle to within $1 \%$ or at most a few percent (Dumont \& Levasseur-Regourd 1978; Leinert \& Pitz 1989), except for subtle effects associated with the scattering of sunlight on the electrons of the interplanetary plasma (Richter et al. 1982). However, seasonal variations occur because of the motion of the observer in heliocentric distance and with respect to the symmetry plane of interplanetary dust cloud (by the annual motion of the earth or the orbital motion of the space probe). The colour of the zodiacal light is similar to solar colour from $0.2 \mu \mathrm{m}$ to $2 \mu \mathrm{m}$, with a moderate amount of reddening with respect to the sun (see Fig. 39). Beyond these wavelengths, the thermal emission of interplanetary dust gradually takes over, the emission being about equal to the scattering part at 3.5 $\mu \mathrm{m}$ (Berriman et al. 1994). In general the zodiacal light is smoothly distributed, small-scale structures appearing only at the level of a few percent.

At present, the overall brightness distribution and polarisation of zodiacal light have been most completely, with the largest sky coverage determined in the visual. The infrared maps obtained by the DIRBE experiment on satellite COBE (see Sect. 8.5) from $1.25 \mu \mathrm{m}$ to $240 \mu \mathrm{m}$ provide excellent data, with relative accuracies of $1 \%$ to $2 \%$ at least for the wavelengths between $1.25 \mu \mathrm{m}$ and $100 \mu \mathrm{m}$. Their absolute accuracy is estimated to $\approx 5 \%$ for wavelengths $\leq 12 \mu \mathrm{m}$ and $\approx 10 \%$ for the longer wavelengths. But these maps are limited to the range in solar elongations of $\epsilon=94^{\circ} \pm 30^{\circ}$. An impression of the accuracy achieved in the visual is obtained by comparing the best available ground-based map (Levasseur-Regourd \& Dumont 1980) with space probe results from Pioneer 10 (Toller \& Weinberg 1985) and Helios A/B (Leinert et al. 1982) in Fig. 34. Among these, e.g. the calibration of the Helios zodiacal light photometers was extensive enough to predict before launch the count rates for bright stars observed in flight to within a few percent, and to propose the same correction to solar $U-B$ and $B-V$ colours (Leinert et al. 1981) as the dedicated solar measurements of Tüg \& Schmidt-Kaler (1982). However the deviation between the three zodiacal light data sets is larger than suggested by this precision, typically $10 \%$, and up to $20 \%$. The deviation appears to be more systematic than statistical in nature. We conclude that the zodiacal light in the visual is known to an accuracy of $10 \%$ at best, about half of which uncertainty is due to multiplicative errors like calibration (including the definition of what a $V=10 \mathrm{mag}$ solar analog G2V star exactly looks like).

In the ultraviolet, the maps of zodiacal light brightness and polarisation are less complete than in the visual, and the calibration is more difficult. In lack of convincingly better information, we assume the overall distribution of zodiacal light brightness at these wavelengths to be the same as in the visual. This, of course, is only a convenient approximation to hardly better than $\approx 20 \%$. Figures 35 and 36 show that this assumption nevertheless gives a reasonable description of the IRAS zodiacal light measure- ments at elongation $\epsilon=90^{\circ}$ (Vrtilek \& Hauser 1995) and an acceptable approximation to the $10.9 \mu \mathrm{m}$ and $20.9 \mu \mathrm{m}$ rocket measurements of Murdock \& Price (1985) along the ecliptic over most of the elongation range. Therefore, in the infrared, it also may be used in those areas where direct infrared measurements are not available.

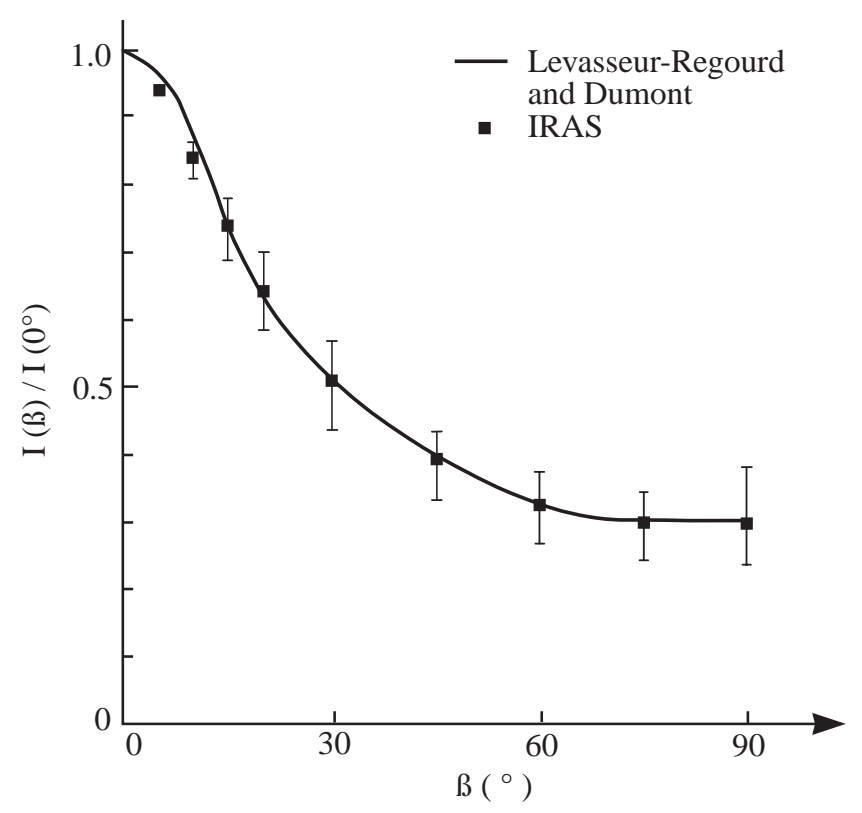

Fig. 35. Comparison of the out-of-ecliptic decrease of zodiacal light brightness at elongation $90^{\circ}$ as measured from ground at $502 \mathrm{~nm}$ (Levasseur-Regourd \& Dumont 1980) and by IRAS (Vrtilek \& Hauser 1995). The IRAS measurements are represented here by their annual average. The squares give the average of the profiles at $12 \mu \mathrm{m}, 25 \mu \mathrm{m}$ and $60 \mu \mathrm{m}$, the bars given with the IRAS measurements show the range covered by the profiles at the different wavelengths, with the measurements at $60 \mu \mathrm{m}$ delineating the lower and the measurements at $12 \mu \mathrm{m}$ the upper envelope

In this spirit, we now want to give the reader the information necessary to get the mentioned estimates of zodiacal light brightness on the basis of the brightness table for visual wavelengths. To this end we write the observed zodiacal light brightness $I_{\mathrm{ZL}}$ for a given viewing direction, position of the observer and wavelength of observation in acceptable approximation (i.e. more or less compatible with the uncertainties of the results) as a product

$$
\begin{aligned}
I_{\mathrm{ZL}}= & f_{R} \cdot I\left(\lambda-\lambda_{\odot}, \beta\right) \cdot f_{\mathrm{abs}} \cdot f_{\mathrm{co}} \cdot f_{\mathrm{SP}} \\
& {\left[S 10_{\odot}, \text { respectively } \mathrm{W} \mathrm{m}{ }^{-2} \mathrm{sr}_{\mu}^{-1} \mathrm{~m}^{-1} \text { or } \mathrm{MJy} / \mathrm{sr}\right] }
\end{aligned}
$$

where

$$
\begin{aligned}
& -I\left(\lambda-\lambda_{\odot}, \beta\right) \text { is the map of zodiacal light brightness in } \\
& \text { the visual for a position in the symmetry plane at } 1 \\
& \text { AU (Table } 16, \text { resp. Table } 17)
\end{aligned}
$$




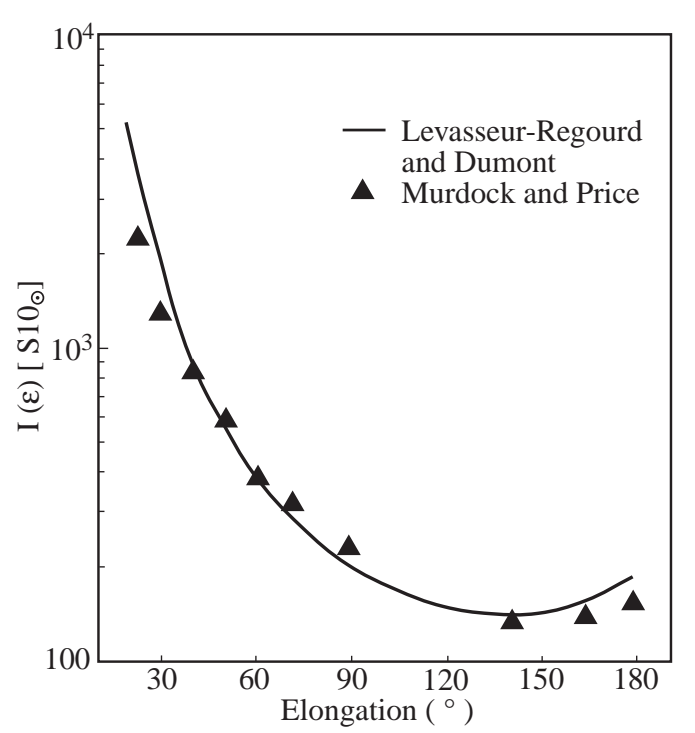

Fig. 36. Comparison of zodiacal light brightness profile along the ecliptic as measured by Levasseur-Regourd \& Dumont (1980) at $502 \mathrm{~nm}$ and by a rocket flight (Murdock \& Price 1985) at $10.9 \mu \mathrm{m}$ and $20.9 \mu \mathrm{m}$. The rocket data for the two wavelength bands have been averaged and normalised to the ground-based measurements at an elongation of $60^{\circ}$. For definition of the $S 10_{\odot}$ unit see Sect. 2

- $f_{\text {abs }}$ transforms from $500 \mathrm{~nm}$ to the wavelength dependent absolute brightness level of the map.

$-f_{\text {co }}$ gives the differential wavelength dependence (i.e. the colour with respect to a solar spectrum), including a colour dependent correction of the map. This factor is applicable from $0.25 \mu \mathrm{m}$ to $2.5 \mu \mathrm{m}$ when the brightness is wanted in $S 10_{\odot}$ units, starting from the value at $500 \mathrm{~nm}$ (Table 16).

- $f_{\mathrm{SP}}$ describes the influence of the position of the observer with respect to the Symmetry Plane of interplanetary dust on the observed brightness. This effect is discussed at length in Sect. 8.7.

- $f_{R}$ gives the dependence on heliocentric distance $\mathbf{R}$.

In the following sections $8.2-8.7$ we provide the quantitative information needed to use the unifying approximate Eq. (14) but also present present individual original results and topics not directly related to it. Section 8.8 discusses the structures present in the zodiacal light on the level of several percent, and Sect. 8.9 indicates how the observed zodiacal light brightness depends on the position of the observer in the solar system.

\subsection{Heliocentric dependence}

This section gives information which allows us to estimate the factor $f_{R}$. Leinert et al. 1980) found the visual bright-
Table 15. Heliocentric gradient of physical properties of interplanetary dust (scattering properties are given for a scattering angle of $90^{\circ}$ )

\begin{tabular}{lccc}
\hline & Value at 1 AU & $\begin{array}{c}\text { Gradient } \\
\text { (power law) }\end{array}$ & $\begin{array}{c}\text { Range } \\
\text { (in AU) }\end{array}$ \\
\hline Density & $10^{-19} \mathrm{~kg} / \mathrm{m}^{3}$ & $-0.93 \pm 0.07$ & $1.1-1.4$ \\
Temperature & $260 \pm 10 \mathrm{~K}$ & $-0.36 \pm 0.03$ & $1.1-1.4$ \\
Albedo & $0.08 \pm 0.02$ & $-0.32 \pm 0.05$ & $1.1-1.4$ \\
& (from IRAS) & & \\
Polarisation & $0.30 \pm 0.03$ & $+0.5 \pm 0.1$ & $0.5-1.4$ \\
$(0.5 \mu \mathrm{m}$, local) & & & \\
\hline
\end{tabular}

ness to increase with decreasing heliocentric distance for all elongations between $16^{\circ}$ and $160^{\circ}$ as

$$
\frac{I(\mathrm{R})}{I(1 \mathrm{AU})}=R^{-2.3 \pm 0.1} \text {. }
$$

In this same range the Helios experiment (Leinert et al. 1982) observed the degree of polarisation to increase with increasing heliocentric distance as

$$
\frac{p(\mathrm{R})}{p(1 \mathrm{AU})}=R^{+0.3 \pm 0.05} \text {. }
$$

In the outer solar sytem, for $1.0 \mathrm{AU}<R<3.3$ AU, Pioneer 10 (Toller \& Weinberg 1985, see also Hanner et al. 1976) found a decrease with heliocentric distance which can be summarised as

$$
\frac{I(\mathrm{R})}{I(1 \mathrm{AU})}=R^{-2.5 \pm 0.2}
$$

neglecting the correction for Pioneer 10's changing distance from the symmetry plane (compare Table 33). Such a steepening is expected to result if there is less interplanetary dust outside the asteroid belt than extrapolated from the inner solar system (van Dijk et al. 1988; Hovenier \& Bosma 1991).

Similarly simple expressions for the thermal infrared cannot be given, since the thermal emission of interplanetary dust

- depends on the temperature $T(\mathrm{R})$ of the dust grains via Planck's function, which is highly nonlinear and therefore

- critically depends on wavelength.

Infrared observations from positions in the inner or outer solar system are not yet available. Estimates therefore have to be based on model predictions (see Sect. 8.9). Examples for such, to varying degrees physical or simply parameterising models are to be found, e.g. in Röser \& Staude (1978), Murdock \& Price (1985), Deul \& Wolstencroft (1988), Rowan-Robinson et al. (1990), Reach 
Table 16. Zodiacal light brightness observed from the Earth (in $S 1 \odot_{\odot}$ ) at $500 \mathrm{~nm}$. Towards the ecliptic pole, the brightness as given above is $60 \pm 3 S 10_{\odot}$. The table is an update of the previous work by Levasseur-Regourd \& Dumont (1980). The values remain the same but for a slight relative increase, both for the region relatively close to the Sun, and for high ecliptic latitudes. The previous table is completed in the solar vicinity, up to $15^{\circ}$ solar elongation. Intermediate values may be obtained by smooth interpolations, although small scale irregularities (e.g. cometary trails) cannot be taken into account

\begin{tabular}{rrrrrrrrrrr}
\hline$\beta^{\circ}$ & 0 & 5 & 10 & 15 & 20 & 25 & 30 & 45 & 60 & 75 \\
$\lambda-\lambda \circ$ & & & & & & & & & & \\
\hline 0 & & & & 2450 & 1260 & 770 & 500 & 215 & 117 & 78 \\
5 & & & & 2300 & 1200 & 740 & 490 & 212 & 117 & 78 \\
10 & & & 3700 & 1930 & 1070 & 675 & 460 & 206 & 116 & 78 \\
15 & 9000 & 5300 & 2690 & 1450 & 870 & 590 & 410 & 196 & 114 & 78 \\
20 & 5000 & 3500 & 1880 & 1100 & 710 & 495 & 355 & 185 & 110 & 77 \\
25 & 3000 & 2210 & 1350 & 860 & 585 & 425 & 320 & 174 & 106 & 76 \\
30 & 1940 & 1460 & 955 & 660 & 480 & 365 & 285 & 162 & 102 & 74 \\
35 & 1290 & 990 & 710 & 530 & 400 & 310 & 250 & 151 & 98 & 73 \\
40 & 925 & 735 & 545 & 415 & 325 & 264 & 220 & 140 & 94 & 72 \\
45 & 710 & 570 & 435 & 345 & 278 & 228 & 195 & 130 & 91 & 70 \\
60 & 395 & 345 & 275 & 228 & 190 & 163 & 143 & 105 & 81 & 67 \\
75 & 264 & 248 & 210 & 177 & 153 & 134 & 118 & 91 & 73 & 64 \\
90 & 202 & 196 & 176 & 151 & 130 & 115 & 103 & 81 & 67 & 62 \\
105 & 166 & 164 & 154 & 133 & 117 & 104 & 93 & 75 & 64 & 60 \\
120 & 147 & 145 & 138 & 120 & 108 & 98 & 88 & 70 & 60 & 58 \\
135 & 140 & 139 & 130 & 115 & 105 & 95 & 86 & 70 & 60 & 57 \\
150 & 140 & 139 & 129 & 116 & 107 & 99 & 91 & 75 & 62 & 56 \\
165 & 153 & 150 & 140 & 129 & 118 & 110 & 102 & 81 & 64 & 56 \\
180 & 180 & 166 & 152 & 139 & 127 & 116 & 105 & 82 & 65 & 56 \\
\hline
\end{tabular}

Table 17. Zodiacal light brightness observed from the Earth (in SI units). This table is identical to the previous one, but for the unit: the values are given in $10^{-8} \mathrm{~W} \mathrm{~m}^{-2} \mathrm{sr}^{-1} \mu \mathrm{m}^{-1}$, for a wavelength of $0.50 \mu \mathrm{m}$. The multiplication factor is $1.2810^{-8}$ $\mathrm{W} \mathrm{m}{ }^{-2} \mathrm{sr}^{-1} \mu \mathrm{m}^{-1}$ (see Table 2 in Sect. 2). Towards the ecliptic pole, the brightness as given above is $77 \pm 10^{-8} \mathrm{~W} \mathrm{~m}^{-2} \mathrm{sr}^{-1}$ $\mu \mathrm{m}^{-1}$. This table (adapted from Levasseur-Regourd 1996) still needs to be multiplied by a corrective factor $F_{\text {co }}$ for use at other wavelengths, in order to take into account the solar spectrum. This table has been added for direct use by those who are not familiar with magnitude related units

\begin{tabular}{rrrrrrrrrrr}
\hline$\beta^{\circ}$ & 0 & 5 & 10 & 15 & 20 & 25 & 30 & 45 & 60 & 75 \\
$\lambda-\lambda_{\circ}$ & & & & & & & & & & \\
\hline 0 & & & & 3140 & 1610 & 985 & 640 & 275 & 150 & 100 \\
5 & & & & 2940 & 1540 & 945 & 625 & 271 & 150 & 100 \\
10 & & & 4740 & 2470 & 1370 & 865 & 590 & 264 & 148 & 100 \\
15 & 11500 & 6780 & 3440 & 1860 & 1110 & 755 & 525 & 251 & 146 & 100 \\
20 & 6400 & 4480 & 2410 & 1410 & 910 & 635 & 454 & 237 & 141 & 99 \\
25 & 3840 & 2830 & 1730 & 1100 & 749 & 545 & 410 & 223 & 136 & 97 \\
30 & 2480 & 1870 & 1220 & 845 & 615 & 467 & 365 & 207 & 131 & 95 \\
35 & 1650 & 1270 & 910 & 680 & 510 & 397 & 320 & 193 & 125 & 93 \\
40 & 1180 & 940 & 700 & 530 & 416 & 338 & 282 & 179 & 120 & 92 \\
45 & 910 & 730 & 555 & 442 & 356 & 292 & 250 & 166 & 116 & 90 \\
60 & 505 & 442 & 352 & 292 & 243 & 209 & 183 & 134 & 104 & 86 \\
75 & 338 & 317 & 269 & 227 & 196 & 172 & 151 & 116 & 93 & 82 \\
90 & 259 & 251 & 225 & 193 & 166 & 147 & 132 & 104 & 86 & 79 \\
105 & 212 & 210 & 197 & 170 & 150 & 133 & 119 & 96 & 82 & 77 \\
120 & 188 & 186 & 177 & 154 & 138 & 125 & 113 & 90 & 77 & 74 \\
135 & 179 & 178 & 166 & 147 & 134 & 122 & 110 & 90 & 77 & 73 \\
150 & 179 & 178 & 165 & 148 & 137 & 127 & 116 & 96 & 79 & 72 \\
165 & 196 & 192 & 179 & 165 & 151 & 141 & 131 & 104 & 82 & 72 \\
180 & 230 & 212 & 195 & 178 & 163 & 148 & 134 & 105 & 83 & 72 \\
\hline
\end{tabular}


Table 18. Zodiacal light polarization observed from the Earth (in percent) The table provides the values for linear polarisation (Levasseur-Regourd 1996). Circular polarisation of zodiacal light is negligible. Positive values correspond to a direction of polarisation ( $\boldsymbol{E}$ vector) perpendicular to the scattering plane (Sun-Earth-scattering particles), negative values correspond to a direction of the polarisation in the scattering plane. Towards the ecliptic pole, the degree of polarisation as given above is $19 \pm 1$ percent. The negative values noticed in the Gegenschein region correspond to a parallel component greater than the perpendicular component, as expected for the scattering by irregular particles at small phase angles

\begin{tabular}{rrrrrrrrrrr}
\hline$\beta^{\circ}$ & 0 & 5 & 10 & 15 & 20 & 25 & 30 & 45 & 60 & 75 \\
$\lambda-\lambda_{\circ}$ & & & & & & & & & & \\
\hline 0 & 1 & & & 8 & 10 & 11 & 12 & 16 & 19 & 20 \\
5 & & & & 9 & 10 & 11 & 12 & 16 & 19 & 20 \\
10 & & & 11 & 11 & 12 & 13 & 14 & 17 & 19 & 20 \\
15 & 13 & 13 & 13 & 13 & 13 & 14 & 15 & 17 & 19 & 20 \\
20 & 14 & 14 & 14 & 15 & 15 & 15 & 15 & 17 & 19 & 20 \\
25 & 15 & 15 & 16 & 16 & 16 & 16 & 16 & 18 & 19 & 20 \\
30 & 16 & 16 & 16 & 16 & 16 & 17 & 17 & 18 & 19 & 20 \\
35 & 17 & 17 & 17 & 17 & 17 & 17 & 17 & 18 & 20 & 20 \\
40 & 17 & 17 & 17 & 17 & 18 & 18 & 18 & 19 & 20 & 20 \\
45 & 18 & 18 & 18 & 18 & 18 & 18 & 18 & 19 & 20 & 20 \\
60 & 19 & 19 & 19 & 19 & 19 & 20 & 20 & 20 & 20 & 20 \\
75 & 18 & 18 & 18 & 18 & 18 & 19 & 19 & 19 & 19 & 19 \\
90 & 16 & 16 & 16 & 16 & 16 & 16 & 17 & 18 & 18 & 19 \\
105 & 12 & 12 & 12 & 12 & 13 & 13 & 14 & 15 & 17 & 19 \\
120 & 8 & 8 & 9 & 9 & 9 & 10 & 11 & 13 & 15 & 18 \\
135 & 5 & 5 & 5 & 6 & 6 & 7 & 8 & 11 & 14 & 17 \\
150 & 2 & 2 & 2 & 3 & 3 & 4 & 5 & 8 & 12 & 16 \\
165 & -2 & -2 & -1 & -1 & 0 & 2 & 3 & 7 & 11 & 16 \\
180 & 0 & -2 & -3 & -2 & -1 & 0 & 2 & 6 & 11 & 16 \\
\hline
\end{tabular}

(1988, 1991), Reach et al. (1996a), Dermott et al. (1996a); see also the discussion of several of these models by Hanner (1991). Present knowledge on the most important physical input parameters is summarised in Table 15, mostly taken from Levasseur-Regourd (1996). Note that "local" polarisation does not mean the zodiacal light polarisation observed locally at the Earth, but the polarisation produced by scattering under $90^{\circ}$ in a unit volume near the Earth's orbit. The gradients (power law exponents in heliocentric distance $R$ ) have been derived from brightness measurements at 1 AU using an inversion method called "nodes of lesser uncertainty" (Dumont \& Levasseur-Regourd 1985). The one directly observed physically relevant quantity in the infrared is the colour temperature of the zodiacal light. At elongation $\epsilon=104^{\circ}$ the colour temperature has been measured between $5 \mu \mathrm{m}$ and $16.5 \mu \mathrm{m}$ from the infrared satellite ISO to be $261.5 \pm 1.5 \mathrm{~K}$ (Reach et al. 1996b). In this wavelength range, the spectrum of the zodiacal light closely followed blackbody emission. See also the discussion of an infrared zodiacal light model in Sect. 8.5.

\subsection{Zodiacal light at $1 A U$ in the visual}

First we give here the values for the zodiacal light at $500 \mathrm{~nm}$ (the possible minute difference to $502 \mathrm{~nm}$, to which the data of Levasseur-Regourd \& Dumont (1980) refer, is neglected). Brightnesses are expressed in $S 10_{\odot}$ units. At $500 \mathrm{~nm}(\Delta \lambda=10 \mathrm{~nm})$ we have

$$
\begin{aligned}
1 S 10_{\odot} & =1.2810^{-8}\left[\mathrm{Wm}^{-2} \mathrm{sr}^{-1} \mu^{-1}\right] \\
& \text { or } \\
1 S 10_{\odot} & =1.2810^{-9}\left[\mathrm{erg} \mathrm{cm} \mathrm{cm}^{-2} \mathrm{~s}^{-1} \mathrm{sr}^{-1} \AA^{-1}\right] .
\end{aligned}
$$

\subsubsection{Pole of the ecliptic}

The annually averaged brightness and degree of polarisation and the polarised intensity $I_{\text {pol }}$ at the ecliptic poles at $500 \mathrm{~nm}$ result as (Levasseur-Regourd \& Dumont 1980; Leinert et al. 1982)

$$
\begin{gathered}
I_{\mathrm{ZL}}\left(\beta=90^{\circ}\right)=60 \pm 3 S 10 \odot \\
p_{\mathrm{ZL}}\left(\beta=90^{\circ}\right)=0.19 \pm 0.01 \\
I_{\mathrm{pol} \mathrm{ZL}}\left(\beta=90^{\circ}\right)=11.3 \pm 0.3 S 10_{\odot} .
\end{gathered}
$$

For completeness we note that the polarized intensity appears to be very much agreed upon, while many of the space experiments (Sparrow \& Ney 1968; Sparrow \& Ney 1972a,b; Levasseur \& Blamont 1973; Frey et al. 1974; Weinberg \& Hahn 1980) tend to find $I_{Z L}$ lower by about $10 \%$ and $p_{\text {ZL }}$ correspondingly higher. But for uniformity of reference within the zodiacal light map below we recommend use of the numbers given above.

\subsubsection{Maps}

Because of the approximate symmetry of the zodiacal light with respect to the ecliptic (resp. symmetry plane) and also with respect to the helioecliptic meridian (sunecliptic poles-antisolar point) only one quarter of the celestial sphere has to be shown. We present the groundbased brightness map for $500 \mathrm{~nm}$ in three ways:

1. Figure 37, taken from Dumont \& Sanchéz (1976) gives the original data in graphical form and allows quick orientation.

2. Table 16, based on the results of Levasseur-Regourd \& Dumont (1980) contains a smoothed tabulation of these (basically same) data in steps of $5^{\circ}$ to $15^{\circ}$ in $\lambda-\lambda_{\odot}$ and $\beta$.

3. Table 17 is identical to Table 16 , except that the brightness now is given in physical units.

The zodiacal light tables given here deviate somewhat from the original earthbound data sets, which were limited to elongation $>30^{\circ}$, because they were subject to additional smoothing, and because they also give a smooth 


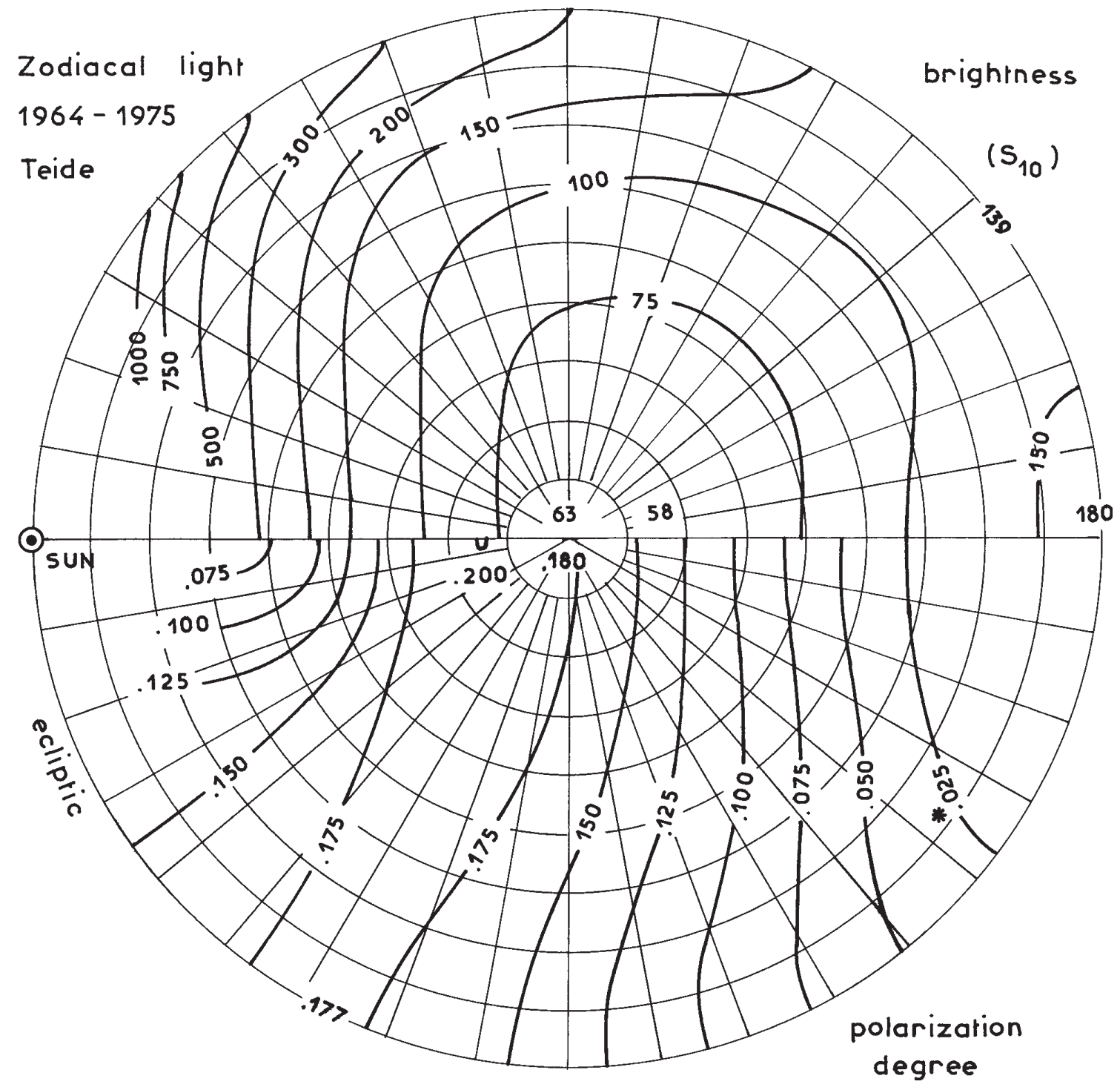

Fig. 37. Annually averaged distribution of the zodiacal light over the sky in differential ecliptic coordinates. Upper half: zodiacal light brightness $I_{\mathrm{ZL}}\left(S 10_{\odot}\right)$, lower half: degree of polarisation of zodiacal light. The circumference represents the ecliptic, the ecliptic pole is in the center, and the coordinates $\lambda-\lambda_{\odot}$ and $\beta$ are drawn in intervals of $10^{\circ}$. The "** indicates a line of lower reliability. From Dumont \& Sanchéz (1976)

connection to two measurements closer to the sun: the results obtained by Helios A/B (Leinert et al. 1982) and those of a precursor rocket flight (Leinert et al. 1976) for small elongations $\left(\epsilon<30^{\circ}\right)$. For interpolation, if the smaller $5^{\circ}$ spacing is needed, still the table in LevasseurRegourd \& Dumont (1980) can be used. In addition, Table 18 gives a map of zodiacal light polarisation, structured in the same way as Tables 16 and 17 .

For these maps, the errors in polarisation are about $1 \%$. The errors in brightness are $10-15 S 10 \odot$ for low values and $5 \%-10 \%$ for the higher brightnesses.
8.4. Wavelength dependence and colour with respect to the sun

The wavelength dependence of the zodiacal light generally follows the solar spectrum from $0.2 \mu \mathrm{m}$ to $\approx 2 \mu \mathrm{m}$. However, detailed study shows a reddening of the zodiacal light with respect to the sun. The thermal emission longward of $3 \mu \mathrm{m}$, as mentioned already in Sect. 8.2, can be approximated by a diluted blackbody radiation. This will bediscussed in more detail in Sect. 8.5. A recent determination of the temperature of this radiation gives the value of $261.5 \pm 1.5 \mathrm{~K}$ (Reach et al. 1996b). 


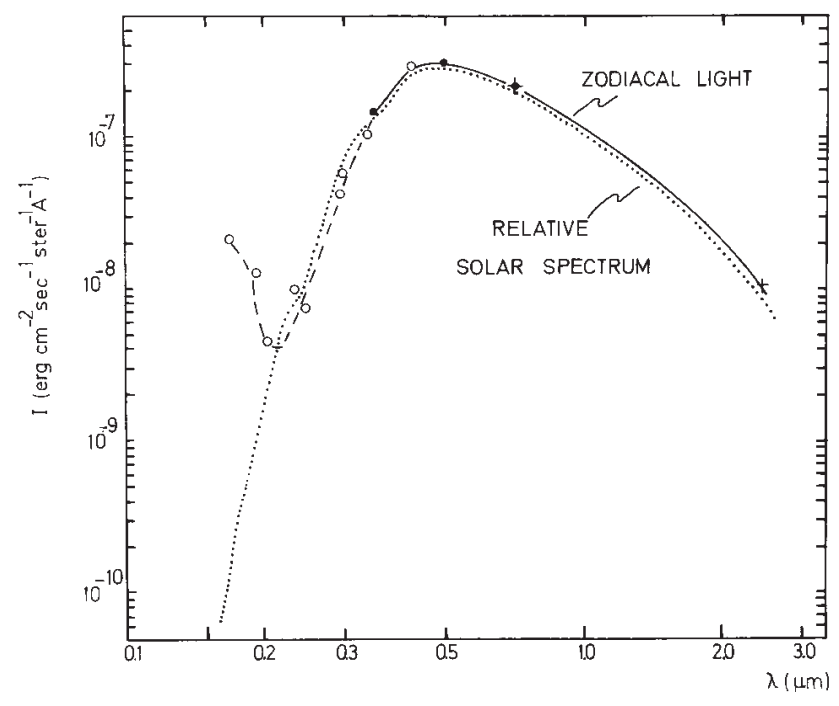

Fig. 38. Broadband spectrum of the zodiacal light. The shown observations are by Frey et al. (1974, •), Hofmann et al. (1973, $+)$, Nishimura et al. $(1973, \nabla)$ and Lillie $(1972, \circ)$. From Leinert (1975)

Table 19. Zodiacal light at $\epsilon=90^{\circ}$ in the ecliptic

\begin{tabular}{llll}
\hline$\lambda(\mu \mathrm{m})$ & $S 10 \odot$ & $\mathrm{W} \mathrm{m}^{-2} \mathrm{sr}^{-1} \mu \mathrm{m}^{-1}$ & $\mathrm{MJy} / \mathrm{sr}$ \\
\hline 0.2 & & $2.510^{-8}$ & \\
0.3 & 162 & $5.310^{-7}$ & \\
0.4 & 184 & $2.210^{-6}$ & \\
0.5 & 202 & $2.610^{-6}$ & \\
$0.7\left(R_{\mathrm{J}}\right)$ & 220 & $2.010^{-6}$ & \\
$0.9\left(I_{\mathrm{J}}\right)$ & 233 & $1.310^{-6}$ & \\
1.0 & 238 & $1.210^{-6}$ & \\
$1.2(J)$ & & $8.110^{-7}$ & 0.42 \\
$2.2(K)$ & & $1.710^{-7}$ & 0.28 \\
$3.5(L)$ & & $5.210^{-8}$ & 0.21 \\
$4.8(M)$ & & $1.210^{-7}$ & 0.90 \\
12 & & $7.510^{-7}$ & 36 \\
25 & & $3.210^{-7}$ & 67 \\
60 & & $1.810^{-8}$ & 22 \\
100 & & $3.210^{-9}$ & 10.5 \\
140 & & $6.910^{-10}$ & 4.5 \\
\hline
\end{tabular}

Figure 38 gives an impression of the spectral flux distribution of the zodiacal light at elongation $\epsilon=80^{\circ}$ in the ecliptic. It emphasises the closeness to the solar spectrum from $0.2 \mu \mathrm{m}$ to $2 \mu \mathrm{m}$. Note that at wavelengths $\lambda<200 \mathrm{~nm}$ the intensity levels expected for a solar-type zodiacal light spectrum are quite low, therefore difficult to establish (see Sect. 8.6).

\subsubsection{Wavelength dependence - absolute level}

This section gives information which allows us to estimate the factor $f_{\text {abs }}$.

From the ultraviolet to near-infrared, if zodiacal light brightness is given in $S 10_{\odot}$ units and the zodiacal light spectrum were solar-like, then we would have simply $f_{\mathrm{abs}}=1.0$.

If the zodiacal light brightness again is expressed in $S 10_{\odot}$ units but its reddening is taken into account, we still take $f_{\text {abs }}=1.0$ and put the reddening into the colour correction factor $f_{\text {co }}$ (see the following section).

If the zodiacal light brightness is given in physical units, $f_{\text {abs }}$ gives the factor by which the absolute level of brightness changes from $\lambda=500 \mathrm{~nm}$ to a given wavelength. Because best defined observationally at an elongation of $\epsilon=90^{\circ}$ in the ecliptic, the factors $f_{\text {abs }}$ should be used for that viewing direction. Table 19 already implicitly contains these factors, since it gives the wavelength dependent brightnesses $I_{\mathrm{ZL}}(\lambda)=I_{\mathrm{ZL}}(500 \mathrm{~nm}) \times f_{\mathrm{abs}}$, for the $90^{\circ}$ points in the ecliptic. (Where appropriate, the factor $f_{\text {co }}$ has also been included). For the infrared emission this brightness is taken from the COBE measurements (see Sect. 8.5) and added here for completeness and easy comparability.

\subsubsection{Colour effects - elongation-dependent reddening}

This section gives information which allows us to estimate the factor $f_{\text {co }}$. This factor applies to the ultraviolet to near-infrared part of the spectrum only. Since it deviates from unity by less than $20 \%$ from $350 \mathrm{~nm}$ to $800 \mathrm{~nm}$, neglecting it (i.e. assuming a strictly solar spectrum) may be acceptable in many applications. Otherwise one has to go through the somewhat clumsy colour correction detailed below.

It is convenient to express the colour of zodiacal light as a colour ratio which linearly measures the deviation of zodiacal light from the the solar spectrum:

$$
C\left(\lambda_{1}, \lambda_{2}\right)=\frac{I_{\mathrm{ZL}}\left(\lambda_{1}\right) / I_{\odot}\left(\lambda_{1}\right)}{I_{\mathrm{ZL}}\left(\lambda_{2}\right) / I_{\odot}\left(\lambda_{2}\right)}
$$

and which, for $\lambda_{1}<\lambda_{2}$, is related to the colour indices $(C I)$ by

$$
C I_{\mathrm{ZL}}-C I_{\odot}=-2.5 \log C\left(\lambda_{1}, \lambda_{2}\right) .
$$

We compile in Fig. 39 measurements of the colour of the zodiacal light with respect to the solar spectrum. There is quite some disagreement in detail, but also a trend for a general reddening which is stronger at small elongations $\left(\epsilon \approx 30^{\circ}\right)$. To be specific, we decide on the basis of Fig. 39, on the following reddening relations (straight lines in this log-linear presentation and giving particular weight to the Helios measurements):

$$
\epsilon<30^{\circ}: \quad f_{\mathrm{co}}=f_{\mathrm{co}-30}
$$



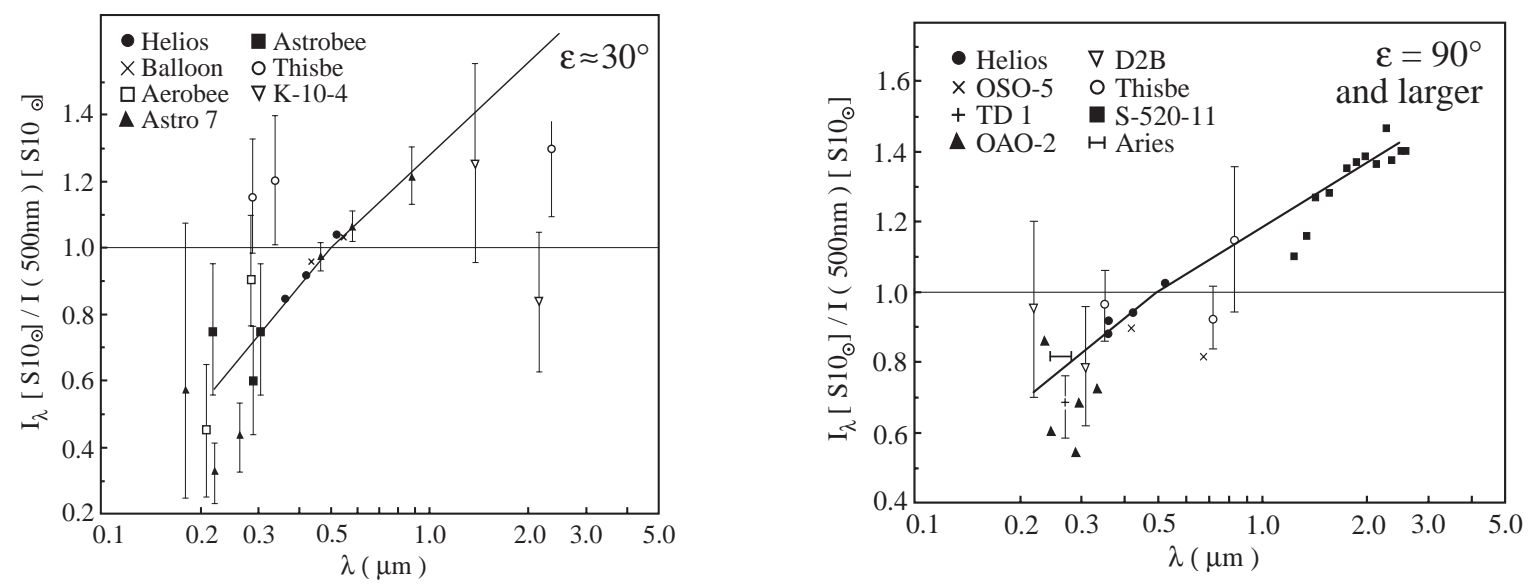

Fig. 39. Reddening of the zodiacal light according to colour measurements by various space-borne and balloon experiments. Left: at small elongations; right: at large elongations. The quantity plotted is the ratio of zodiacal light brightness at wavelength $\lambda$ to zodiacal light brightness at wavelength $500 \mathrm{~nm}$, normalised by the same ratio for the sun (i.e. we plot the colour ratio $C(\lambda$, $500 \mathrm{~nm}$ ). Reddening corresponds to a value of this ratio of $<1.0$ for $\lambda<500 \mathrm{~nm}$ and $>1.0$ for $\lambda>500 \mathrm{~nm}$. The thick solid line represents the adopted reddening (Eq. (22)). The references to the data points are: Leinert et al. (1981) (Helios), Vande Noord (1970) (Balloon), Feldman (1977) (Aerobee rocket), Pitz et al. (1979) (Astro 7 rocket), Cebula \& Feldman (1982) (Astrobee rocket), Frey et al. (1977) (Balloon Thisbe), Nishimura (1973) (rocket K-10-4), Sparrow \& Ney (1972a,b) (OSO-5), Morgan et al. (1976) (TD-1), Lillie (1972) (OAO-2), Maucherat-Joubert et al. (1979) (D2B), Matsuura et al. (1995) (rocket S-520-11), Tennyson et al. (1988) (Aries rocket)

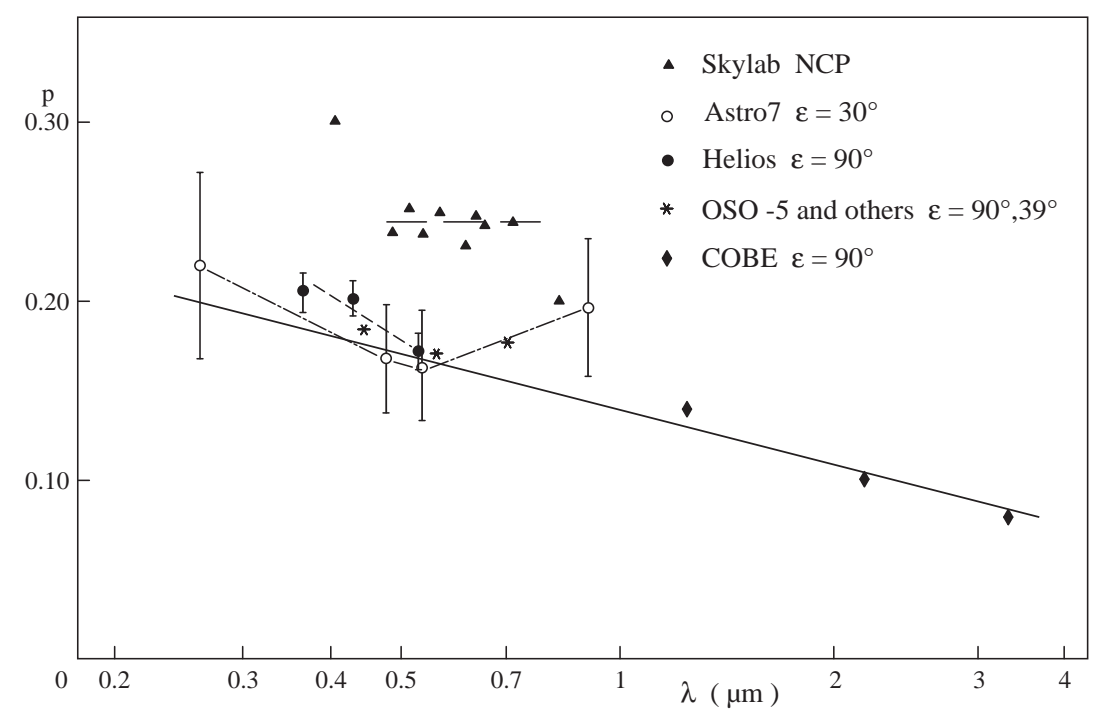

Fig. 40. Wavelength dependence of polarisation observed at different positions in the zodiacal light. Filled triangles - Skylab at the north celestial pole (Weinberg \& Hahn 1979); open circles: rocket Astro 7 at elongation $\epsilon=30^{\circ}$ (Pitz et al. 1979); dots: Helios at $\beta=16^{\circ}, \epsilon=90^{\circ}$ (Leinert et al. 1982); diamonds: COBE measurements (Berriman et al. 1994); stars: an average of three similar results (OSO-5, $\epsilon=90^{\circ}$, Sparrow \& Ney 1972; balloon at $\epsilon=30^{\circ}$, Vande Noord 1970; ground-based at $\epsilon=39^{\circ}$, Wolstencroft \& Brandt 1967). Note: it is the wavelength dependence within each group which matters. The solid line shows the approximation (24) to the wavelength dependence of $p$ 


$$
\begin{aligned}
\epsilon=30^{\circ}: \quad f_{\mathrm{co}-30}= & 1.0+1.2 \times \log \left(\frac{\lambda}{500 \mathrm{~nm}}\right) \\
& 220 \mathrm{~nm} \leq \lambda \leq 500 \mathrm{~nm} \\
= & 1.0+0.8 \times \log \left(\frac{\lambda}{500 \mathrm{~nm}}\right) \\
& 500 \mathrm{~nm} \leq \lambda \leq 2.5 \mu \mathrm{m} \\
\epsilon=90^{\circ}: \quad f_{\mathrm{co}-90}= & 1.0+0.9 \times \log \left(\frac{\lambda}{500 \mathrm{~nm}}\right) \\
& 220 \mathrm{~nm} \leq \lambda \leq 500 \mathrm{~nm} \\
= & 1.0+0.6 \times \log \left(\frac{\lambda}{500 \mathrm{~nm}}\right) \\
& 500 \mathrm{~nm} \leq \lambda \leq 2.5 \mu \mathrm{m} \\
\epsilon>90^{\circ}: \quad f_{\mathrm{co}}= & f_{\mathrm{co}-90 .}
\end{aligned}
$$

Here, $f_{\text {co }}=1.0$ coresponds to solar colour, while a reddening results in $f_{\text {co }}<1.0$ for $\lambda<500 \mathrm{~nm}$ and in $f_{\text {co }}>1.0$ for $\lambda>500 \mathrm{~nm}$.

For intermediate values of $\epsilon, f_{\text {co }}$ can be interpolated. The curves for the assumed colour in Fig. 39 are made to closely fit the Helios data, where the $U B V(363 \mathrm{~nm}$, $425 \mathrm{~nm}, 529 \mathrm{~nm}$ ) colours (Leinert et al. 1982), again expressed as colour ratios, were

$$
\begin{aligned}
& \frac{I_{V}}{I_{B}}=1.14-5.510^{-4} \cdot \epsilon\left(^{\circ}\right) \\
& \frac{I_{B}}{I_{U}}=1.11-5.010^{-4} \cdot \epsilon\left(^{\circ}\right) .
\end{aligned}
$$

Obviously the colour ratio factor $f_{\text {co }}$ cannot be very accurate in the ultraviolet (where measurements don't agree too well) nor beyond $1 \mu \mathrm{m}$ (where partly extrapolation is involved). The situation for $\lambda \leq 220 \mathrm{~nm}$ in the ultraviolet and for the emission part of the zodiacal light are described below in separate sections.

\subsubsection{Wavelength dependence of polarisation}

The available zodiacal light polarisation measurements between $0.25 \mu \mathrm{m}$ and $3.5 \mu \mathrm{m}$ fall in two groups (Fig. 40). Most observations in the visual can be represented within their errors by a polarisation constant over this wavelength range. Two quite reliable measurements, on the other hand (by Helios in the visible and by COBE in the near-infrared), show a definite decrease of observed degree of polarisation with wavelength.

In the limited wavelength range from $0.45 \mu \mathrm{m}$ to $0.80 \mu \mathrm{m}$ it is still an acceptable approximation to assume the polarisation of the zodiacal light as independent of wavelength. But overall, the wavelength dependence of polarisation summarised in Fig. 40 has to be taken into account. For an elongation of $90^{\circ}$, to which most of the data in Fig. 40 refer, it can be reasonably represented by the relation (solid line in the figure)

$$
p(\lambda)=0.17+0.10 \cdot \log (\lambda / 0.5 \mu \mathrm{m})]
$$

i.e. by a decrease of $\approx 3 \%$ per factor of two in wavelength. With $p(0.5 \mu \mathrm{m})=0.17$, this can also be written in the form

$$
p(\lambda)=p(0.5 \mu \mathrm{m})[1+0.59 \log (\lambda / 0.5 \mu \mathrm{m})],
$$

which may be applied tentatively also to other viewing directions.

At longer wavelengths, with the transition region occuring between $\approx 2.5 \mu \mathrm{m}$ and $5 \mu \mathrm{m}$, the zodiacal light is dominated by thermal emission and therefore unpolarised. At shorter wavelengths the zodiacal light brightness is very low, and the polarisation is not known (although it may be similar to what we see in the visual spectral range).

Maps of the zodiacal light polarisation at present are available with large spatial coverage for the visual spectral range only. For other wavelength ranges, it is a first approximation to use the same spatial distribution.

\subsection{Zodiacal light in the thermal infrared}

Extensive space-based measurements of the diffuse infrared sky brightness in the infrared have become available over the past 13 years (e.g., Neugebauer et al. 1984 (IRAS); see Beichman 1987 for a review of IRAS results; Murdock \& Price 1985 (ZIP); Boggess et al. 1992 (COBE); Murakami et al. 1996 (IRTS); Kessler et al. 1996 (ISO)). In general, some form of modeling is required to separate the scattered or thermally emitted zodiacal light from other contributions to the measured brightness, though at some wavelengths and in some directions the zodiacal light is dominant. Because the COBE/DIRBE measurements have the most extensive combination of sky, temporal, and wavelength coverage in the infrared, and have been carefully modeled to extract the zodiacal light signal (Reach et al. 1996a; COBE/DIRBE Explanatory Supplement), we largely rely on these results.

The spectral energy distribution of the zodiacal light indicates that the contributions from scattered and thermally emitted radiation from interplanetary dust are about equal near $3.5 \mu \mathrm{m}$ (Spiesman et al. 1995; Matsumoto et al. 1996), where the interplanetary dust (IPD) contribution to the infrared sky brightness is at a local minimum. This turnover is most clearly seen in the data of the near-infrared spectrometer onboard the satellite IRTS (Matsumoto et al. 1996, see Fig. 41). Observations in the range $3-5 \mu \mathrm{m}$ are expected to be neither purely scattering not purely thermal. The thermal spectrum peaks near $12 \mu \mathrm{m}$, and the observed spectral shape for $\lambda<100 \mu \mathrm{m}$ approximates that of a blackbody (for a power law emissivity proportional to $\nu^{-n}$, spectral index $n=0$ ) with a temperature in the range 250 - 290 K (Murdock \& Price 1985; Hauser et al. 1984; Spiesman et al. 1995), depending in part on the direction of observation. As already mentioned, recent results from ISO (Reach et al. 1996b, Fig. 42) fit the $5-16.5 \mu \mathrm{m}$ wavelength range with a blackbody of $T=261.5 \pm 1.5 \mathrm{~K}$. Using 


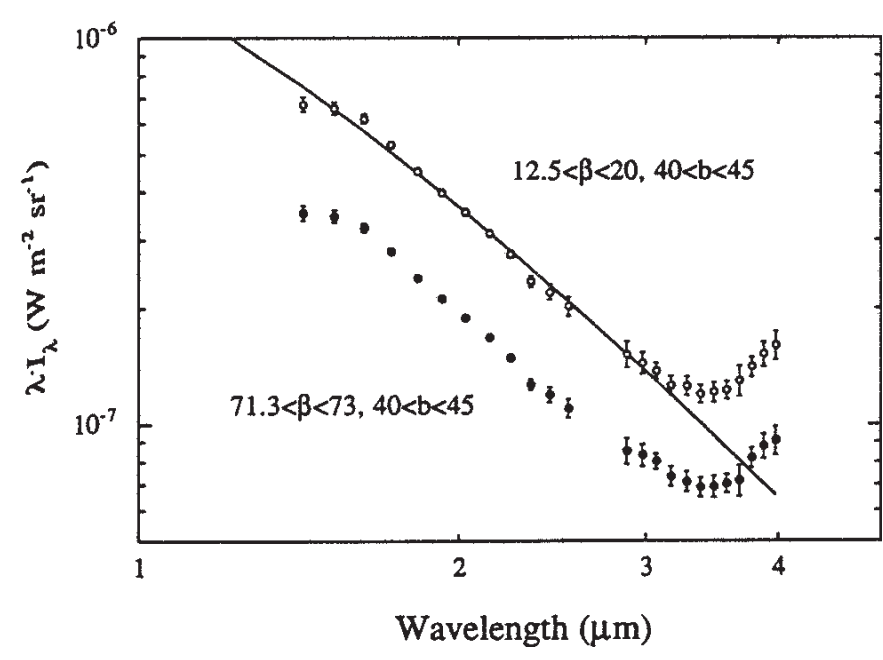

Fig. 41. Near-infrared spectra of the sky brightness measured with the satellite IRTS at low and at high ecliptic latitudes $\beta$. The solid line gives a solar spectrum, normalised to the measurements at low $\beta$ at $1.83 \mu \mathrm{m}$. From Matsumoto et al. (1996)

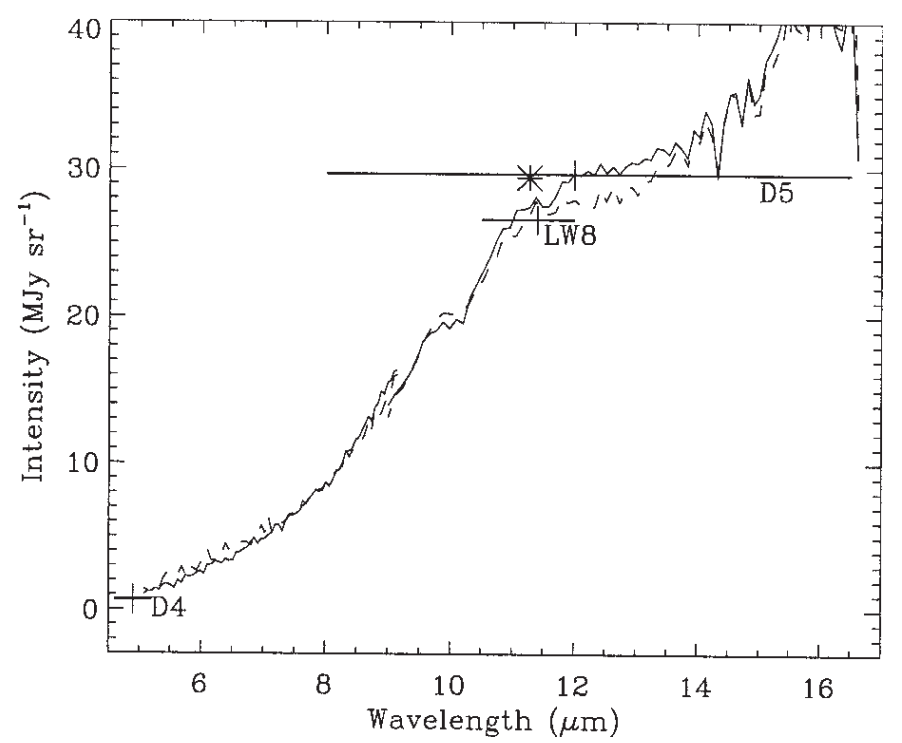

Fig. 42. Spectrum of the zodiacal light from $5 \mu \mathrm{m}$ to $16.5 \mu \mathrm{m}$ as measured with the circular variable filter of the infrared camera (CAM) onboard the ISO satellite. Actually, two separate measur4ements are overplotted. "D4" and "D5" are measurements of the DIRBE/COBE exoeriment shown here for comparison, where the horizontal bar indicates the width of the filters. "LW8" and the asterisk refer to independent measurements obtained with CAM. Note the possible broad emission feature between $9 \mu \mathrm{m}$ and $12 \mu \mathrm{m}$. From Reach et al. (1996b)
COBE/DIRBE data, Reach et al. (1996a) find a slow rolloff of the emissivity in the far-infrared (spectral index $n \approx 0.5$ for $\lambda>100 \mu \mathrm{m})$.

Except near the Galactic plane, the signal due to interplanetary dust dominates the observed diffuse sky brightness at all infrared wavelengths shortward of $\approx 100 \mu \mathrm{m}$. This is illustrated in Fig. 43, which presents COBE/DIRBE observations (0.7 deg resolution) of a strip of sky at elongation $90 \mathrm{deg}$ in 10 photometric bands ranging from $1 \mu \mathrm{m}-240 \mu \mathrm{m}$. The estimated contribution from zodiacal light (based upon the DIRBE model, see below) is also shown at each wavelength in Fig. 43. Even in the far infrared, the contribution from zodiacal light is not necessarily negliglible: Reach et al. (1996a) estimated the fraction of total sky brightness due to zodiacal light at the NGP as roughly $25 \%$ at $240 \mu \mathrm{m}$. Examination of Fig. 43 shows that, although the signal due to interplanetary dust peaks near the ecliptic plane at all wavelengths, the detailed shape of the signal is wavelength-dependent. An analytic empirical relation for the brightness in the thermal infrared at $90^{\circ}$ elongation (based upon IRAS data) has been described by Vrtilek \& Hauser (1995). As already mentioned, the brightness distribution in visual can serve as a first approximation to the brightness distribution in the thermal infrared, if the respective infrared data are not available.

Although the shape of the underlying zodiacal "lower envelope" is clearly visible in the data of Fig. 43, the determination of the zero-level of the zodiacal light in the infrared is difficult. In addition to calibration uncertainties in the sky brightness measurements themselves, contributions from Galactic sources and possibly extragalactic background make this a challenging problem.

A summary of several techniques which have been used to isolate the zodiacal light from other sky signals is documented by Hauser (1988): many involve filtering the data in either the angular or angular frequency domain, leaving the absolute signal level uncertain. Others accomplish removal of the Galactic component via models, e.g. by using the statistical discrete source model of Wainscoat et al. (1992), or by use of correlations with measurements at other wavelengths (e.g., HI; Boulanger \& Perrault 1988). We choose here to quote zodiacal light levels as derived from the DIRBE zodiacal light model, which is based upon a parameterized physical model of the interplanetary dust cloud similar to that used for IRAS (Wheelock et al. 1994, Appendix G). Rather than determining the model parameters by fitting the observed sky brightness, the DIRBE model was derived from a fit to the seasonally-varying component of the brightness in the DIRBE data, since that is a unique signature of the part of the measured brightness arising in the interplanetary dust cloud (Reach et al. 1996a). The model explicitly includes several spatial components (see Sect. 8.8): a large-scale smooth cloud, the dust bands attributed to asteroidal collisions, and the 

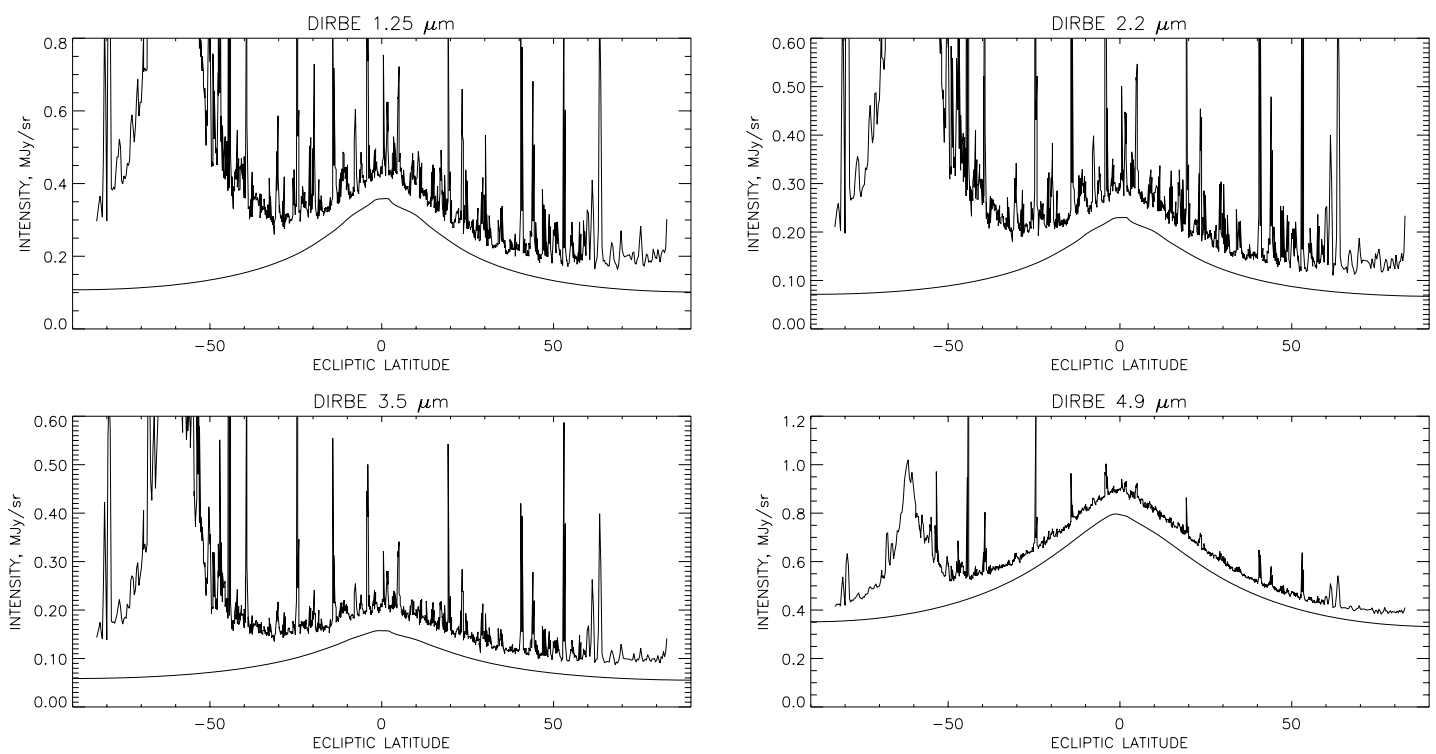

DIRBE $12 \mu \mathrm{m}$
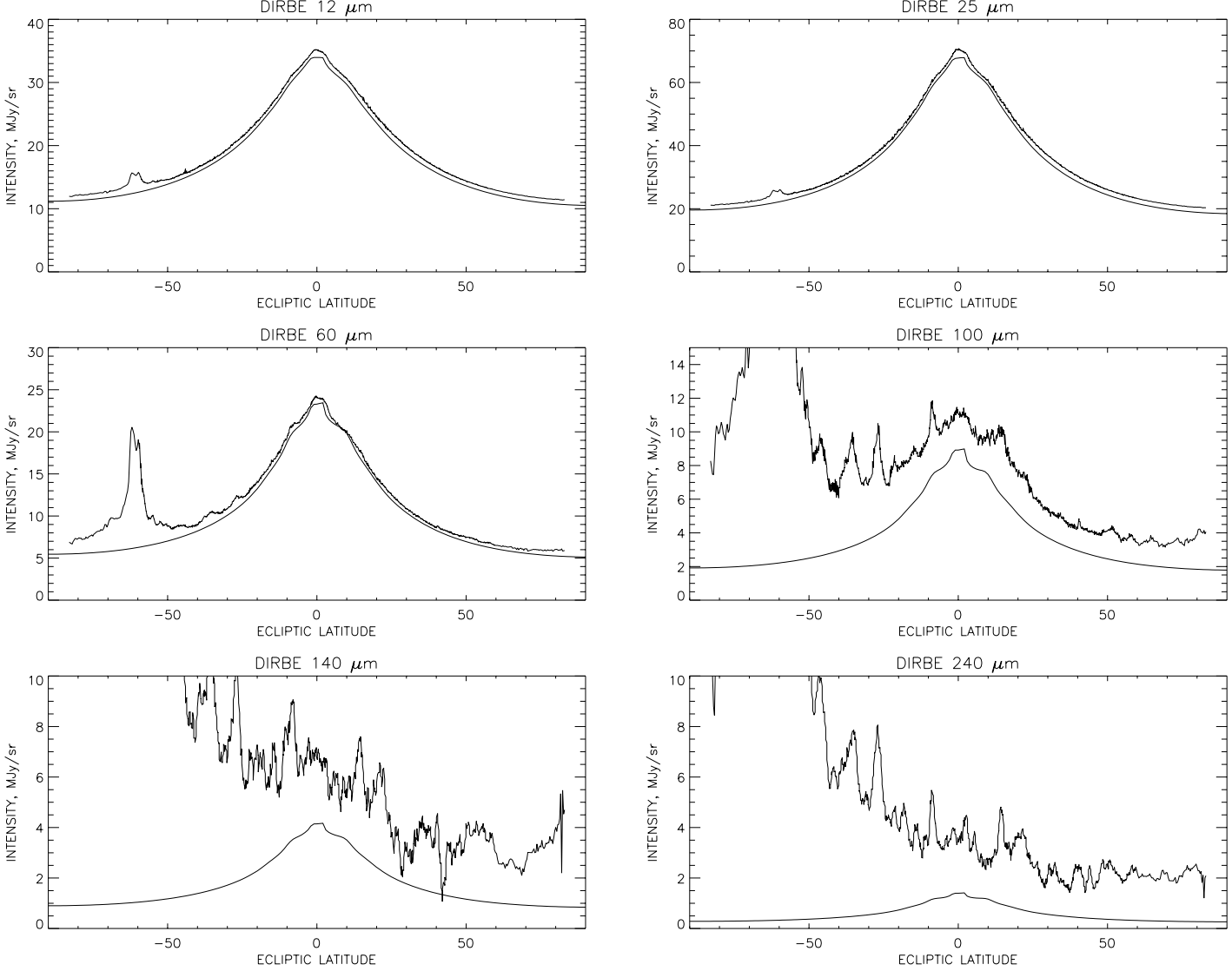

Fig. 43. Example of total IR sky brightness measured by the COBE/DIRBE instrument and brightness contributed by zodiacal light at 10 infrared wavelengths. At each wavelength, the upper curve shows the sky brightness measured by DIRBE on 1990 Jun 19 at solar elongation $90^{\circ}$, ecliptic longitude $179^{\circ}$, as a function of geocentric ecliptic latitude. Because of low signal-to-noise ratio at the longest wavelengths, the $140 \mu \mathrm{m}$ and $240 \mu \mathrm{m}$ data have been averaged and smoothed. The lower curve in each plot is the zodiacal light brightness for this epoch obtained from the DIRBE zodiacal light model. DIRBE is a broad-band photometer: flux densities are given in MJy/sr at the nominal wavelengths of the DIRBE bands, assuming an input energy distribution of the form $\nu I_{\nu}=$ constant 

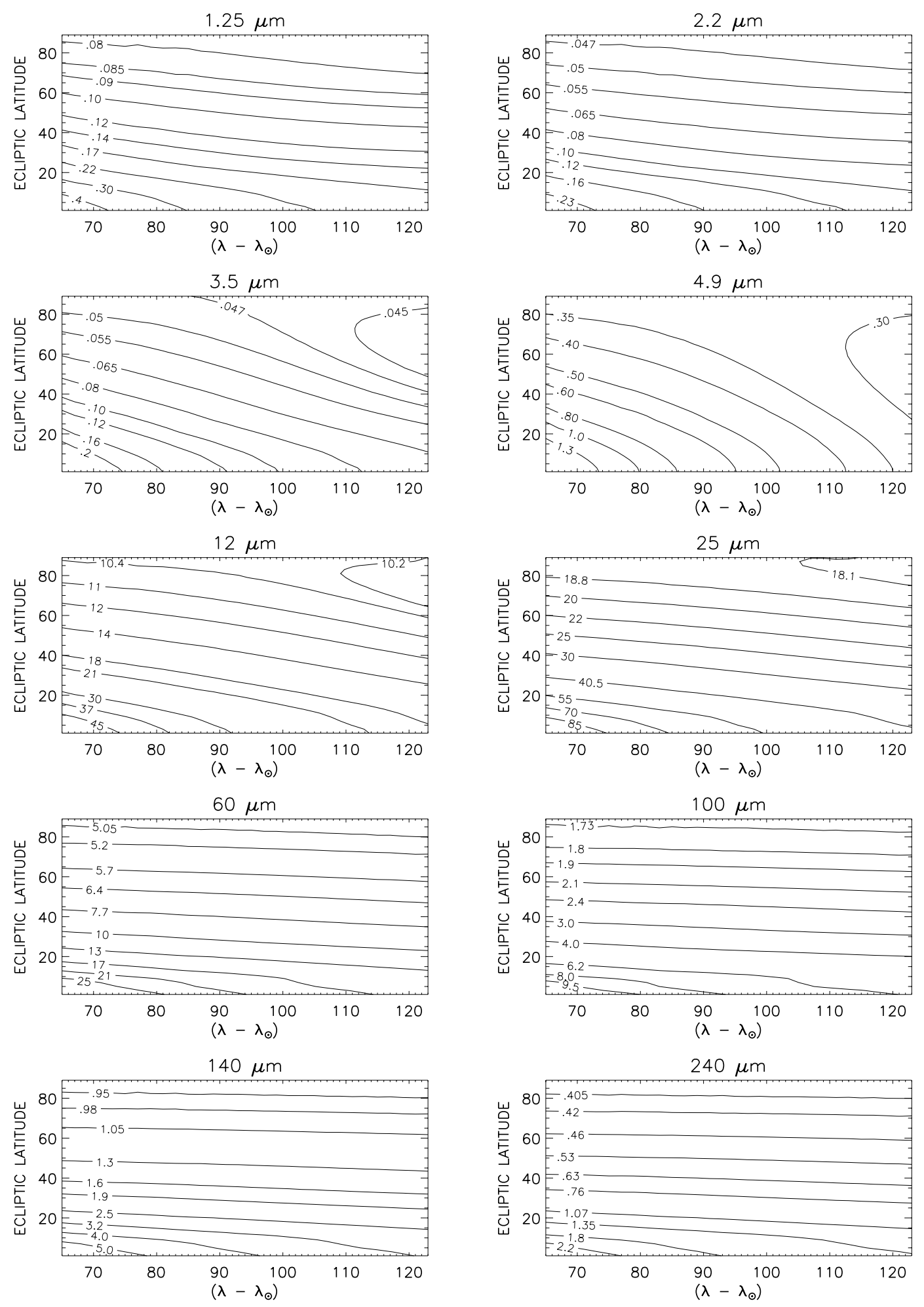

Fig. 44. Contour maps of average zodiacal light brightness in the 10 DIRBE wavebands, as derived from the DIRBE zodiacal light model. Contours are labelled in units of MJy/sr. No color corrections for the broad DIRBE bandwidths have been applied (see DIRBE Explanatory Supplement, Sect. 5.5, for details) 


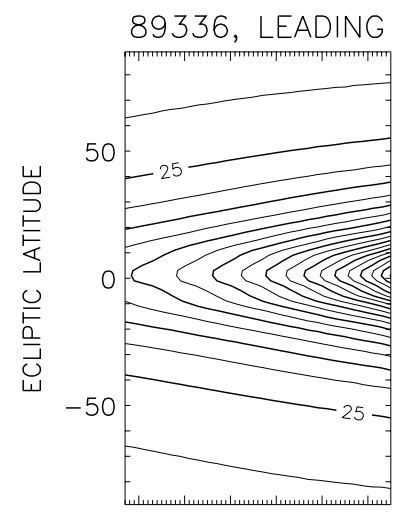

120110100908070

$\left(\lambda-\lambda_{\odot}\right)$

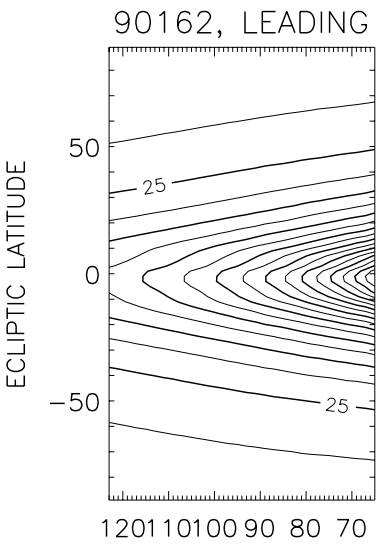

$\left(\lambda-\lambda_{\odot}\right)$

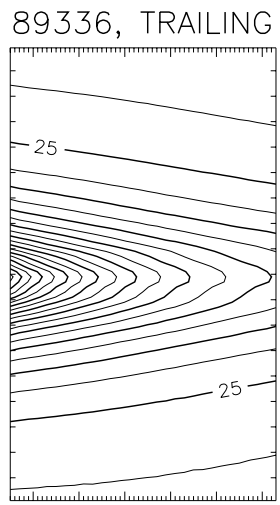

708090100110120

$\left(\lambda-\lambda_{\odot}\right)$

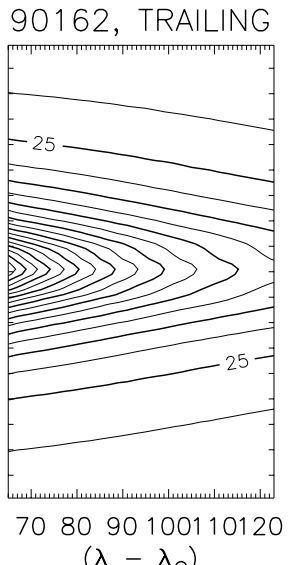

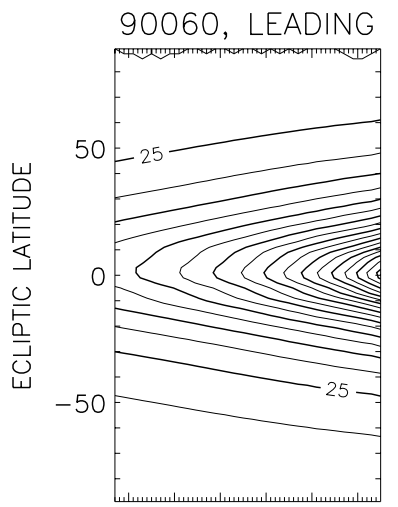

120110100908070

$\left(\lambda-\lambda_{\odot}\right)$

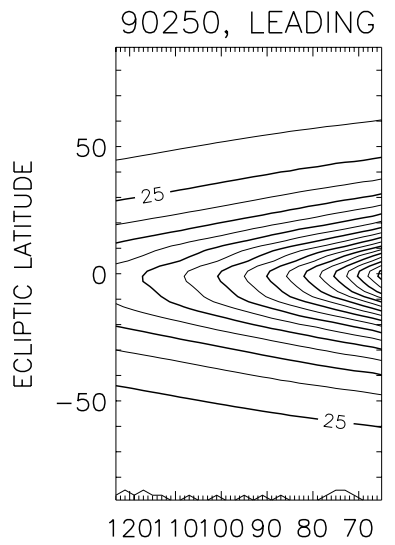

$\left(\lambda-\lambda_{\odot}\right)$

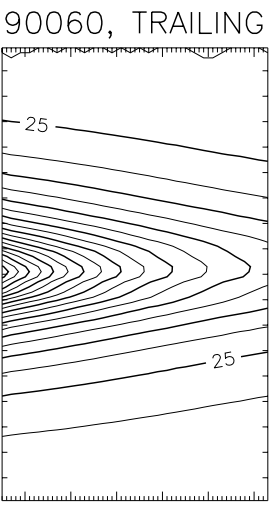

708090100110120

$\left(\lambda-\lambda_{\odot}\right)$

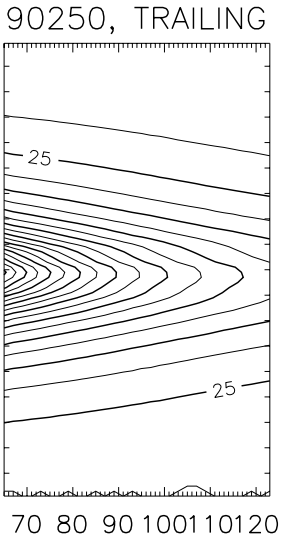

$\left(\lambda-\lambda_{\odot}\right)$

Fig. 45. Contour maps of the zodiacal light brightness at $25 \mu \mathrm{m}$ for four different times of the year, based on the DIRBE zodiacal light model. Contours are given in increments of $5 \mathrm{MJy} / \mathrm{sr}$, with the $25 \mathrm{MJy} / \mathrm{sr}$ level labelled. Each pair of maps shows contours for both the leading side and trailing side of the Earth's orbit. The epoch for each pair is indicated above the map, in the format yyddd, e.g., 89336 is day 336 (Dec. 2) of 1989. Asymmetries between the two sides, as well as changes with epoch, can be seen in these maps. Again, flux densities are given in MJy/sr at the nominal wavelengths of the DIRBE bands, assuming an input energy distribution of the form $\nu I_{\nu}=$ constant. $\left(\lambda-\lambda_{\odot}\right)$ is given from $70^{\circ}$ to $120^{\circ}$ in steps of $10^{\circ}$

resonantly-trapped dust ring near 1 AU. Zodiacal light levels given here are estimated to be accurate to $\approx 10 \%$ for wavelengths of $25 \mu \mathrm{m}$ and shortward, and $\approx 20 \%$ for longer wavelengths. Note that for all DIRBE spectral intensities presented here, the standard DIRBE (and IRAS) convention is used: the calibration is done for a spectrum with $\nu I_{\nu}=$ constant, which means in particular that the effective bandwidth of each DIRBE wavelength band is calculated assuming a source spectrum with this shape. In general, and for accurate work, then a colour correction based upon the actual source spectral shape must be applied (see DIRBE Explanatory Supplement for details).

Figure 44 presents contours of "average" zodiacal light isophotes in geocentric ecliptic coordinates for one quarter of the sky (other quadrants are given by symmetry), as computed from the DIRBE model. Although this average serves as a guideline for the contribution of zodiacal light to the night sky brightness at infrared wavelengths, at no point in time will an Earth-based observer see a zodiacal light foreground exactly resembling these contours. The detailed DIRBE measurements indicate that the individual spatial components of the interplanetary dust cloud possess their own geometry, their own "symmetry plane" and their own temporal variation pattern.

Figure 45 illustrates, again on the basis of the COBE zodiacal light model, the variation in isophotes at $25 \mu \mathrm{m}$ at four different times during the year, corresponding roughly to the times when the Earth is in the symmetry plane of the main dust cloud [days 89336 and 90162] and when it is $90^{\circ}$ further along its orbit [days 90060 and 90250].

Detailed quantitative maps of the DIRBE measurements and zodiacal light model are available from the 


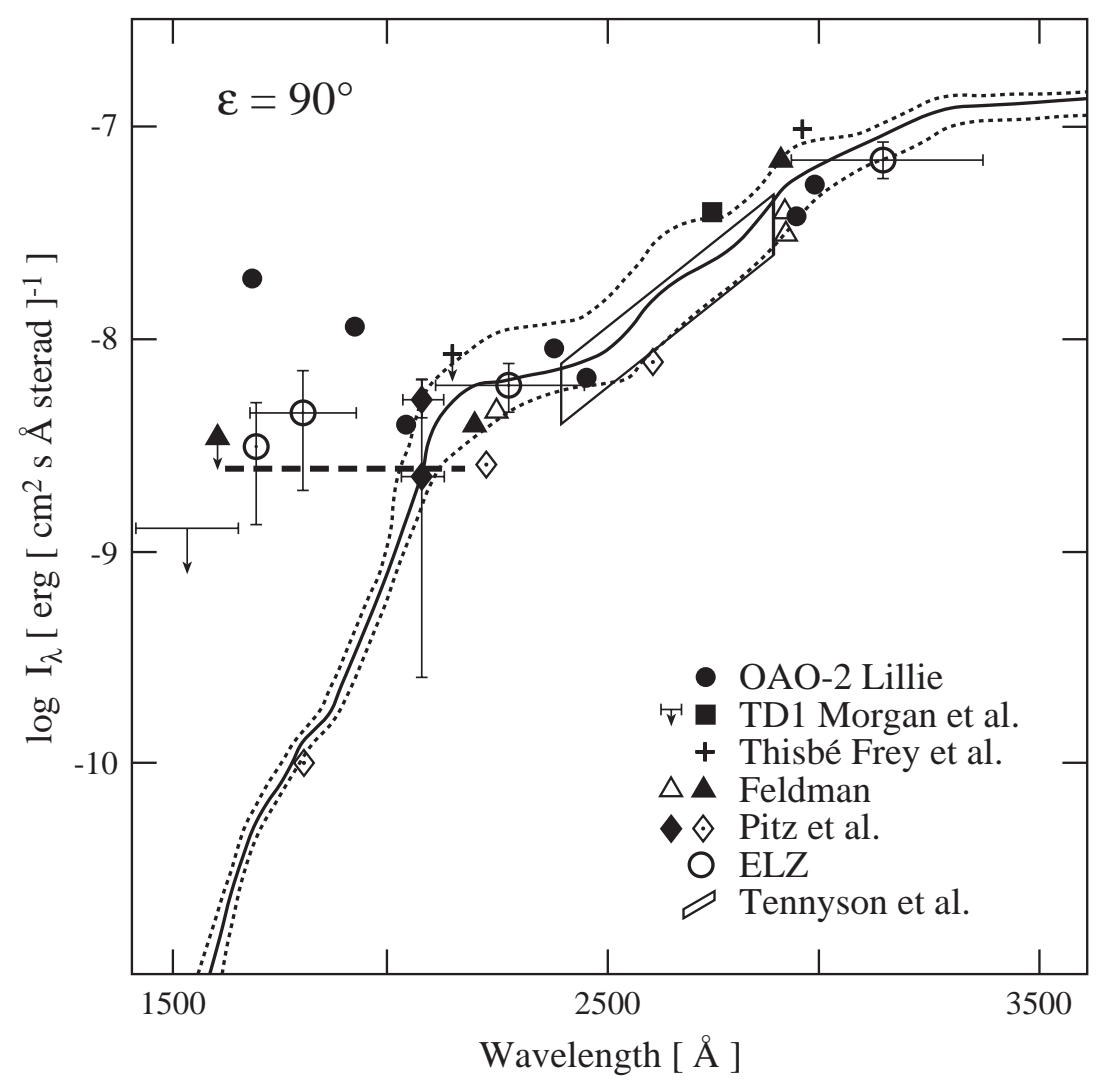

Fig. 46. Ultraviolet zodiacal light measurements at $90^{\circ}$ elongation in the ecliptic in absolute fluxes, compared to the solar spectrum. Measurements from smaller elongations have been transformed to the intensity scale of the figure by assuming the same distribution of zodiacal light brightness over the sky as in the visual. The chosen average zodiacal light brightness for $160 \mathrm{~nm} \leq \lambda \leq 220 \mathrm{~nm}$ is shown as thick broken line. Differences with respect to Fig. 38 result from what is used as solar spectrum in the ultraviolet and from the way in which visual data are compared to ultraviolet measurements. The references to the data points are: Lillie (1972), Morgan (1978), Morgan et al. (1976), Frey et al. (1977), Feldman (1977), Cebula \& Feldman (1982), Pitz et al. (1979) and a reanalysis by Maucherat-Joubert et al. (1979), Maucherat-Joubert et al. (ELZ, 1979), Tennyson et al. (1988). Adapted from Maucherat-Joubert et al. (1979)

NASA National Space Science Data Center in the DIRBE Sky and Zodiacal Atlas. The COBE/DIRBE data products and the Explanatory Supplement are accessible through the COBE Home Page at http:://www.gsfc.nasa.gov/astro/cobe/cobe_home.html on the World Wide Web.

\subsection{Zodiacal light in the ultraviolet $(\lambda<300 \mathrm{~nm})$}

The difficulty with this wavelength range is that here the zodiacal light contribution appears only as a small fraction of the observed background. Available measurements therefore have large error bars or only give upper limits. In addition there is a sharp drop of solar irradiance below $220 \mathrm{~nm}$, by three orders of magnitude until $150 \mathrm{~nm}$. This can be seen in Fig. 46 which summarises available results. The scatter between the observations is very large. Whatever the reason for Lillie's (1972) high values (variation, galactic component, instrumental effects), his results shortward of $\lambda=220 \mathrm{~nm}$ no longer are accepted as originally given. In view of the obvious discrepancies we suggest to accept the following:

$$
\begin{aligned}
I_{\mathrm{ZL}}(\lambda)= & \text { negligible, }<110^{-8} \quad \mathrm{~W} \mathrm{~m}^{-2} \mathrm{sr}^{-1} \mu \mathrm{m}^{-1} \\
& (\text { for } \lambda<160 \mathrm{~nm}) \\
I_{\mathrm{ZL}}(\lambda)= & 2.510^{-8} \frac{I\left[\left(\lambda-\lambda_{\odot}, \beta\right)+I\left(\epsilon, 0^{\circ}\right)\right] / 2}{I\left(90^{\circ}, 0^{\circ}\right)} \\
\mathrm{W} \mathrm{m}^{-2} \mathrm{sr}^{-1} \mu \mathrm{m}^{-1} & \\
& (\text { for } 160 \mathrm{~nm} \leq \lambda<220 \mathrm{~nm}) \\
I_{\mathrm{ZL}}(\lambda)= & \text { of solar spectrum, with reddening } \\
& \text { as given in Sect. } 8.4 .2 \text { above } \\
& (\text { for } 220 \mathrm{~nm} \leq \lambda<300 \mathrm{~nm}) .
\end{aligned}
$$

Here, $I\left(\lambda-\lambda_{\odot}, \beta\right)$ refers to the map of the zodiacal light at $500 \mathrm{~nm}$ given above in Table 16 .

Murthy et al. (1990) from their Space Shuttle experiment found that the colour of the zodiacal light gets bluer with increasing ecliptic latitude between $165 \mathrm{~nm}$ and $310 \mathrm{~nm}$. This would mean, that the zodiacal light is less 
flattened and more symmetrically distributed around the sun at these wavelengths, as also found from OAO-2 (Lillie 1972). This is an important result which should systematically be confirmed. In Eq. (26) we take such an effect qualitatively into account and approximate it by halving the out-of ecliptic decrease with respect to the visible wavelengths (this is what the lengthy fraction does).

At $220 \mathrm{~nm}$ there are now two expressions for the brightness of zodiacal light in Eq. (26), with different out-of ecliptic decrease of brightness. They agree at an intermediate latitude (resp. inclination) of $30^{\circ}-45^{\circ}$. The discontinuity at the other ecliptic latitudes is acceptable, given the large uncertainties of the determination of zodiacal light brightness at these wavelengths.

\subsection{Seasonal variations}

The effects to be discussed in this section have been summarised as factor $f_{\mathrm{SP}}$ in Eq. (14) above.

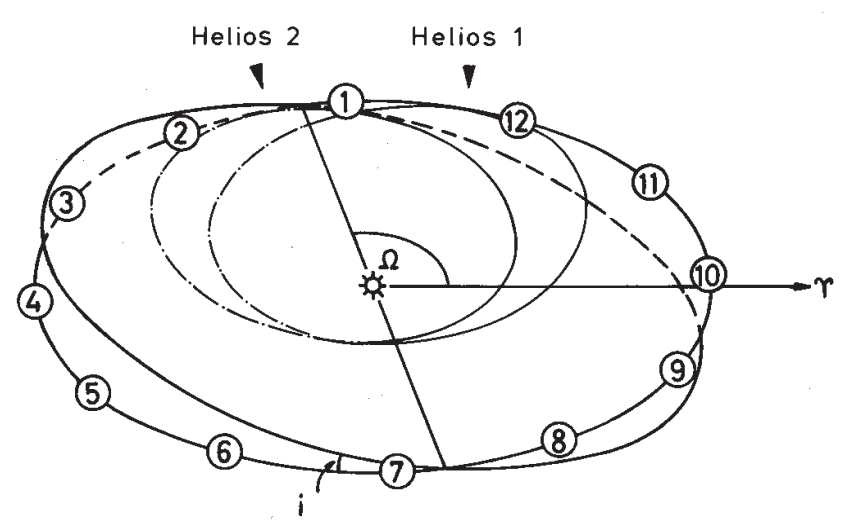

Fig. 47. Geometry of the earth orbit and the symmetry plane of interplanetary dust (with ascending node $\Omega$ and inclination $i$ ). Numbers give the position of the earth at the beginning of the respective month. Also shown are the orbits of the Helios spaceprobes and the direction to the vernal equinox

Seasonal variations of zodiacal light brightness occur for an observer moving with the earth, on the level of $\approx 10 \%$. They result from the orbital motion of the earth through the interplanetary dust cloud, which changes the heliocentric distance (by $2 \mathrm{e}=3.3 \%$ ) and the position of the observer with respect to the symmetry plane of the interplanetary dust distribution (see Fig. 47). (The symmetry plane is a useful concept for describing the interplanetary dust distribution, although in detail it is too simplified: the symmetry properties appear to change with heliocentric distance, see Table 20). The change in heliocentric distance of the observer translates into a brightness increase of about $8 \%$ from aphelion in July to perihelion in January. Otherwise, the effects are different for high and for low ecliptic latitudes. Since the effects are very similar in the visual spectral range and in the infrared, examples from both wavelength ranges will be used to show the effects.

\subsubsection{High ecliptic latitudes}

At high ecliptic latitudes, the main effect is a yearly sinusoidal variation of the brightness with an amplitude of $\approx \pm 10 \%$. This is due to the motion of the earth south and north of the midplane of dust depending on its orbital position. The extrema occur when the earth (the observer) is at maximum elevation above or below the symmetry plane, while the average value is obtained when crossing the nodes. The effect is clearly visible in the broadband optical Helios measurements in the inner solar system (Fig. 48), in the D2A satellite observations at $653 \mathrm{~nm}$ along the earth's orbit (Fig. 49) and in the COBE infrared measurements (Fig. 50) . Of these, the Helios measurements have been corrected for the changing heliocentric distance of the instrument, while in the other data the modulation still contains the $\approx 8 \%$ effect due to the eccentricity of the earth's orbit. The effect of the tilted symmetry plane gradually decreases towards low ecliptic latitudes to $\leq 1 \%$. The brightness changes in low ecliptic latitude observations from the earth or from earthbound satellites then are dominated by the effect of changing heliocentric distance.

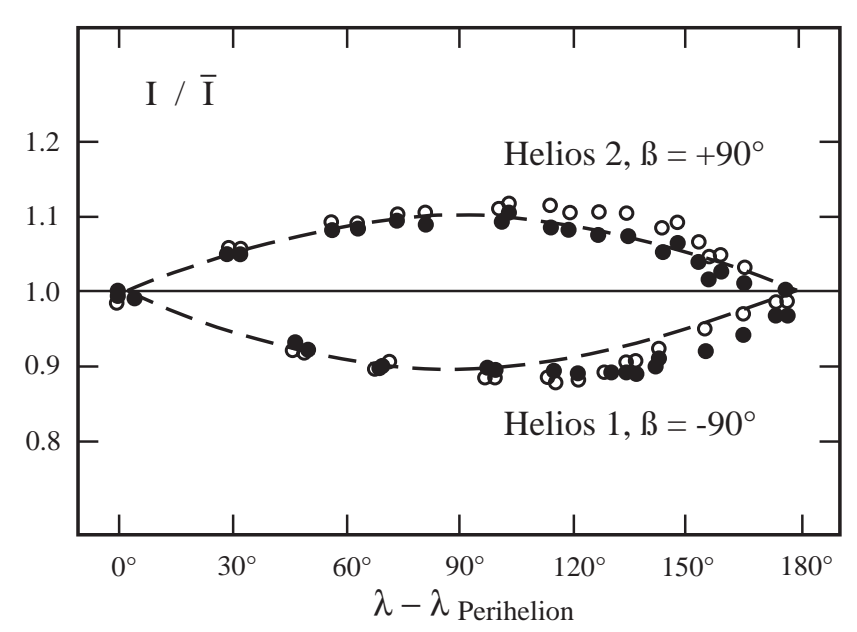

Fig. 48. Change of brightness with ecliptic longitude observed by Helios at the ecliptic poles. The dashed line gives a sinusoidal fit to the data. These observations refer to the inner solar system, from $0.3 \mathrm{AU}$ to $1.0 \mathrm{AU}$. The perihelia of the Helios space probes are at $\lambda \approx 100^{\circ}$. From Leinert et al. (1980b) 


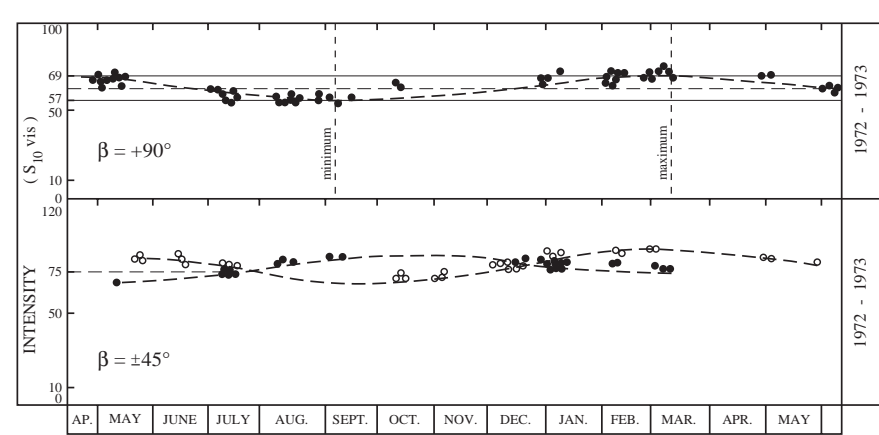

Fig. 49. Yearly variation of zodiacal light brightness at the north ecliptic pole and at $\pm 45^{\circ}$ ecliptic latitude, observed at $653 \mathrm{~nm}$ by the satellite D2A. The dashed line is a prediction for a plane of symmetry coinciding with the invariable plane of the solar system $\left(i=1.6^{\circ}, \Omega=107^{\circ}\right)$, including the effect of changing heliocentric distance. Adapted from Levasseur \& Blamont (1975)

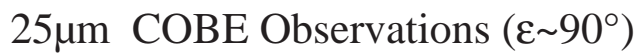

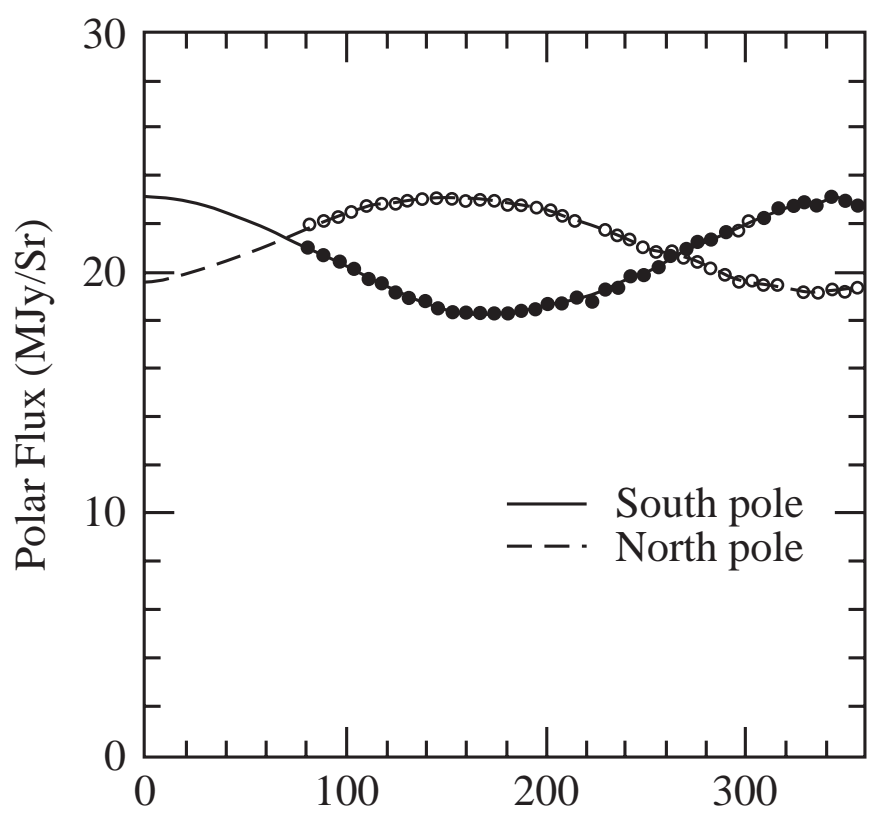

Ecliptic Longitude of Earth

Fig. 50. Yearly brightness variations in the zodiacal light at the ecliptic poles, observed at $25 \mu \mathrm{m}$ by the DIRBE experiment on infrared satellite COBE. The variation is dominated by the effect of the tilt of the symmetry plane but also includes the variation due to the changing heliocentric distance of the earth. From Dermott et al. (1996b)

\subsubsection{Low ecliptic latitudes}

At low ecliptic latitudes, the motion of the earth with respect to the symmetry plane of interplanetary dust mainly leads to a sinusoidal variation in the ecliptic latitude of the peak brightness of the zodiacal light by a few degrees. Figure 51 shows this variation as observed at $25 \mu \mathrm{m}$ from COBE. In these measurements, the remaining yearly peak flux variation of $5-10 \%$ is almost exclusively due to the change in heliocentric distance. Misconi (1977) has used an approximate method to predict the expected position of the brightness maxima in the visible zodiacal light for elongations of $\epsilon=2^{\circ}-180^{\circ}$ (typically, the positions vary by a couple or a few degrees; at elongations $\geq 150^{\circ}$ the approximation he uses gets unreliable).

\subsubsection{Plane of symmetry of interplanetary dust}

The seasonal variations discussed above have repeatedly been used to determine the plane of symmetry of interplanetary dust. This midplane of the interplanetary dust distribution appears to vary with heliocentric distance, as summarised in Table 20, compiled from Reach (1991). For comparison, we give here also inclinations and ascending nodes for Venus, Mars and the invariable plane of the solar system $\left(i=3.4^{\circ}, \Omega=76^{\circ} ; i=1.8^{\circ}, \Omega=49^{\circ} ; i=1.6^{\circ}\right.$, $\Omega=107^{\circ}$ ).

Table 20. Plane of symmetry of interplanetary dust

\begin{tabular}{|c|c|c|c|c|}
\hline Range (AU) & $\Omega\left({ }^{\circ}\right)$ & $i\left({ }^{\circ}\right)$ & Ref. & Remarks \\
\hline \multirow{3}{*}{$\begin{array}{l}0.3-1.0 \\
\quad \approx 1.0\end{array}$} & $87 \pm 4$ & $3.0 \pm 0.3$ & 1 & optical \\
\hline & $96 \pm 15$ & $1.5 \pm 0.4$ & 2 & optical \\
\hline & $79 \pm 3$ & $1.7 \pm 0.2$ & 3 & $\begin{array}{l}\text { infrared } \\
\text { at poles }\end{array}$ \\
\hline$\approx 1.3$ & $55 \pm 4$ & $1.4 \pm 0.1$ & 4 & $\begin{array}{l}\text { infrared } \\
\text { in ecliptic }\end{array}$ \\
\hline$\approx 3$ & $\approx 96$ & $\approx 1.1$ & 5 & $\begin{array}{l}\text { asteroidal } \\
\text { bands }\end{array}$ \\
\hline
\end{tabular}

References: 1) Leinert et al. (1980b) ${ }^{2)}$ Dumont \& LevasseurRegourd (1978) ${ }^{3)}$ Reach (1991) 4) Hauser (1988) ${ }^{5)}$ Sykes (1985).

\subsection{Structures in the zodiacal light}

Notwithstanding the variety of sources contributing to the interplanetary dust population, the zodiacal light in general is quite smooth, and it was found to be stable to $\approx 1 \%$ over more than a decade (Leinert \& Pitz 1989). However, there are fine structures on the brightness level of a few percent, most of which have been detected by the IRAS 

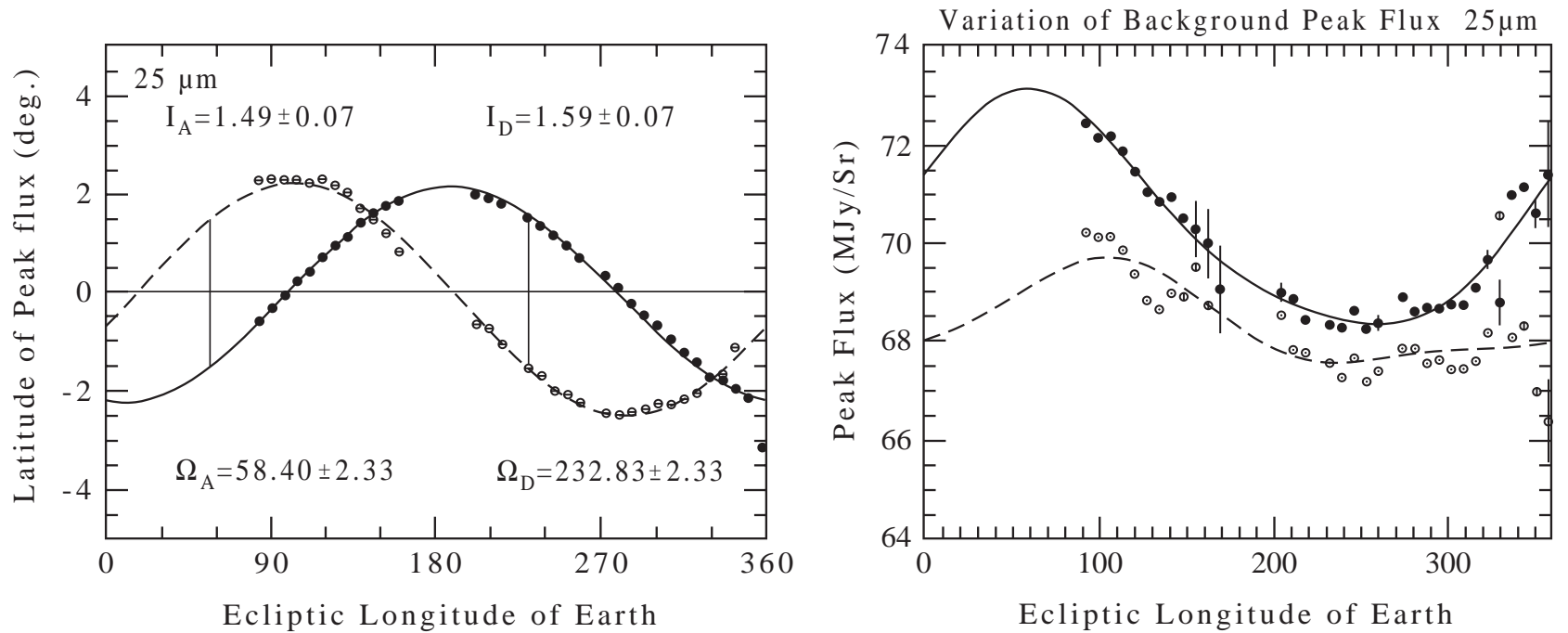

Fig. 51. Yearly variation of the ecliptic latitude of zodiacal light peak brightness (left) and yearly variation of peak brightness (right) observed at $25 \mu \mathrm{m}$ at elongation $\epsilon=90^{\circ}$ By the DIRBE experiment on infrared satellite COBE. Open circles refer to the leading (apex), filled circles to the trailing (antapex) direction. From Dermott et al. (1996a,b)

Table 21. Properties of dust bands from Gaussian fits

\begin{tabular}{|c|c|c|c|}
\hline Band & $12 \mu \mathrm{m}$ & $25 \mu \mathrm{m}$ & $60 \mu \mathrm{m}$ \\
\hline \multicolumn{4}{|c|}{ Peak surface brightness $\left(\mathrm{MJy} \mathrm{sr}^{-1}\right)$} \\
\hline$\gamma$ northern & $0.4 \pm 0.2$ & $1.1 \pm 0.5$ & $0.8 \pm 0.4$ \\
\hline$\alpha, \beta$ northern & $1.1 \pm 0.5$ & $3.0 \pm 1.0$ & $1.5 \pm 0.5$ \\
\hline$\alpha, \beta$ southern & $1.4 \pm 0.3$ & $2.9 \pm 1.2$ & $1.6 \pm 0.6$ \\
\hline$\gamma$ southern & $0.6 \pm 0.3$ & $0.8 \pm 0.3$ & $0.7 \pm 0.4$ \\
\hline \multicolumn{4}{|c|}{ Average geocentric latitude of peak $\left(^{\circ}\right)$} \\
\hline$\gamma$ northern & $9.7 \pm 0.1$ & $9.6 \pm 0.1$ & $9.6 \pm 0.2$ \\
\hline$\alpha, \beta$ northern & $1.4 \pm 0.1$ & $1.4 \pm 0.1$ & $1.4 \pm 0.1$ \\
\hline$\alpha, \beta$ southern & $-1.4 \pm 0.1$ & $-1.4 \pm 0.1$ & $-1.4 \pm 0.1$ \\
\hline$\gamma$ southern & $-9.7 \pm 0.1$ & $-9.6 \pm 0.1$ & $-9.6 \pm 0.1$ \\
\hline \multicolumn{4}{|c|}{ Full width at half maximum brightness $\left({ }^{\circ}\right)$} \\
\hline$\gamma$ northern & $3.3 \pm 1.3$ & $3.7 \pm 1.1$ & $3.2 \pm 1.5$ \\
\hline$\alpha, \beta$ northern & $3.3 \pm 1.1$ & $3.3 \pm 1.2$ & $3.2 \pm 1.2$ \\
\hline$\alpha, \beta$ southern & $3.7 \pm 1.3$ & $3.3 \pm 1.2$ & $3.4 \pm 1.4$ \\
\hline$\gamma$ southern & $2.8 \pm 1.1$ & $3.1 \pm 0.8$ & $3.0 \pm 1.4$ \\
\hline
\end{tabular}

infrared sky survey: asteroidal bands, cometary trails, and a resonant dust ring just outside the Earth's orbit. They are included here because of their physical importance; they also represent upper limits in brightness to any other structures which still might be hidden in the general zodiacal light distribution. The rms brightness fluctuations of the zodiacal light at $25 \mu \mathrm{m}$ have been found by observa- tions from the satellite ISO in a few half-degree fields to be at most $\pm 0.2 \%$ (Ábráham et al. 1997).

\section{Asteroidal bands}

They were seen in the IRAS infrared scans across the ecliptic as bumps in the profile near ecliptic latitude $\beta=0^{\circ}$ and as shoulders at $\beta \approx 10^{\circ}$ (Low et al. 1984, see Fig. 52). The bands near the ecliptic plane have been called $\alpha$ and $\beta$ (counted from ecliptic latitude $\beta=0^{\circ}$ outwards), the ones around $\beta=10^{\circ}$ have been called $\gamma$ bands. Their peak brightness is $1 \%-3 \%$ of the in-ecliptic zodiacal light brightness, their width at half maximum $\approx 2-3^{\circ}$ (Reach 1992, but the detailed values depend on the method actually used to fit the bumps, in this case by Gaussians). They are thought to be the result of major collisions in the asteroid belt, in the Themis and Koronis families for the $\alpha$ and $\beta$ bands, in the Eos family for the higher latitude $\gamma$ bands (Dermott et al. 1984). The collisional debris then is expected to be mainly distributed along the walls of widely opened, slightly tilted, sun-centered cones. Therefore the ecliptic latitudes at which these bands occur vary both with the annual motion of the observer (the earth in most cases) and, at a given date, with the elongation from the sun. Formulae to predict the position of the maximum with help of a simplified geometrical model are given by Reach (1992). Figure 53, resulting from an analysis of the IRAS data, gives a good impression of the resulting yearly sinusoidal latitude variation. Table 21 (taken again from Reach 1992) summarises the average observed properties of the asteroidal dust bands in the case Gaussian fitting is used to measure the bumps in the general distribution of zodiacal light. There must be in addition an underlying 


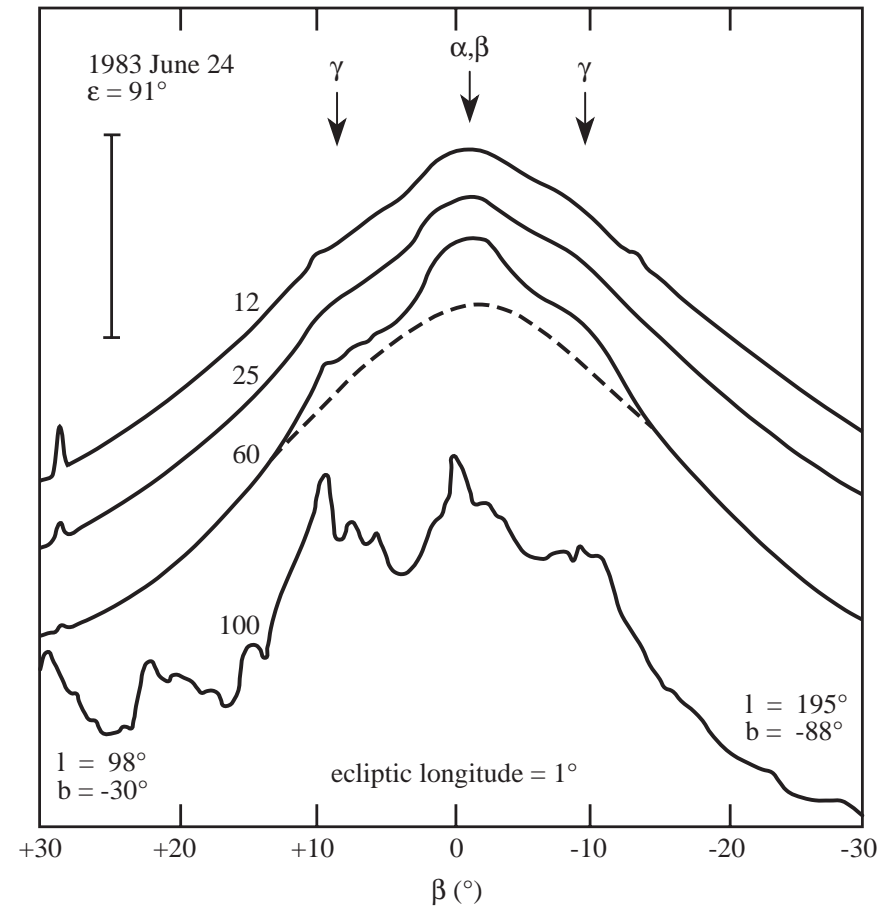

Fig. 52. Scans through the ecliptic at ecliptic longitude $\lambda=$ $1^{\circ}$ on June 24,1983 . The approximate galactic coordinates for the point at $\pm 30^{\circ}$ ecliptic latitude are given. The curves are labelled by the wavelength of measurement in $\mu \mathrm{m}$. A rough calibration is given by the bar at upper left, the length of which corresponds to $12,30,10$ and $6 \mathrm{MJy} / \mathrm{sr}$ in the wavelength bands from $12 \mu \mathrm{m}$ to $100 \mu \mathrm{m}$. The dashed curve illustrates how a completely smooth zodiacal light distribution might have looked. The arrows indicate the positions of the asteroidal bands. The $100 \mu \mathrm{m}$ profile is strongly distorted by thermal emission from interstellar dust ("cirrus"). Adapted from Low et al. (1984)

distribution of asteroidal debris particles of about $10 \%$ of the zodiacal light brightness, which cannot be seen separately from the general zodiacal light. Note that Sykes (1988) resolved the $\alpha$ and $\beta$ bands also into band pairs, with a FWHM of $\approx 0.5^{\circ}$ for each of the components. The claim for eight additional, though weaker bands between $\beta=-22^{\circ}$ and $\beta=+21^{\circ}$ (Sykes 1988) should be taken with reservation and can be neglected here.

\section{Cometary trails}

These trails have been seen in the IRAS infrared sky survey stretching along the orbit of a few periodic comets, which were in the perihelion part of their orbit (Sykes et al. 1986). These were the comets Tempel 2, Encke, Kopff, Tempel 1, Gunn, Schwassmann-Wachmann 1, Churyumov-Gerasimenko and Pons-Winnecke, but also nine faint orphan trails without associated comet were found (Sykes \& Walker 1992). The trails typically extend $10^{\circ}$ behind and $1^{\circ}$ ahead of the comet, their brightness decreasing with increasing distance from the comet. They are

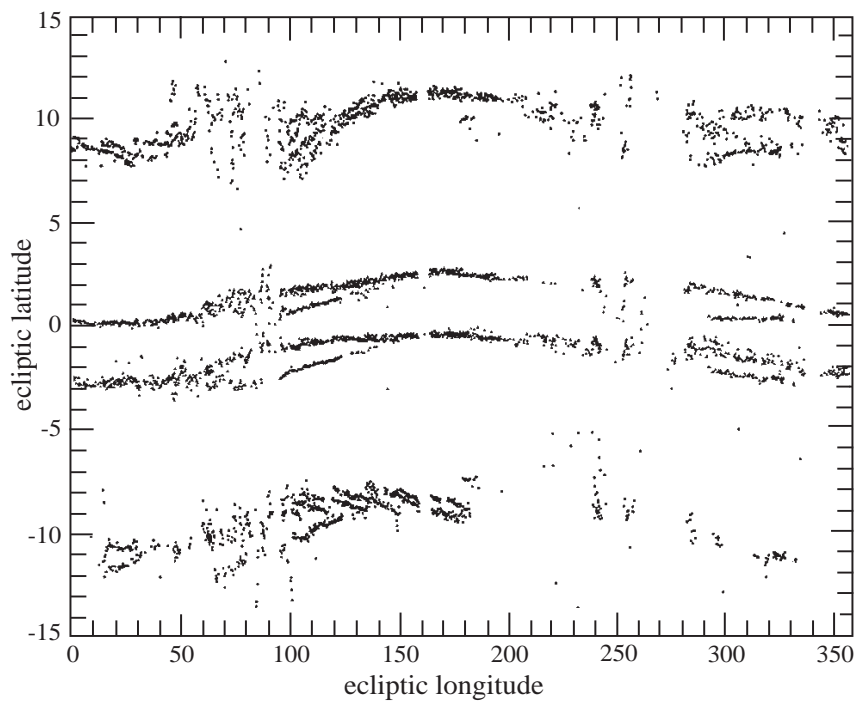

Fig. 53. Observed ecliptic latitude of the peak brightness of the asteroidal bands as function of the ecliptic longitude of the viewing direction (basically as function of the orbital motion of the earth). The expected sinusoidal variation is evident but distorted, since the elongation of the viewing direction was modulated on an approximately monthly timescale, and because observations both east and west of the sun were contained in the data set. Taken from Reach (1992)

thought to consist of roughly mm-sized particles ejected from the comet during times of activity over many years (Sykes et al. 1990). The trails are bright enough to be seen above the zodiacal light only when the comets are near perihelion and the dust in the trails is warm. The width of the trails is about one arcminute, for comet Tempel 2 it has been determined to $45^{\prime \prime} \pm 2^{\prime \prime}(\approx 30000 \mathrm{~km})$. Trail brightnesses are of the order of $1 \%$ of the zodiacal light brightness near the ecliptic. Examples are given in Table 22, taken in shortened form from Sykes \& Walker (1992). Other periodic comets in the perihelion part of their orbit are expected to behave similarly. A new observation of the comet Kopff trail from ISO (Davies et al. 1997) has shown changes in the trail since the observations by IRAS, and measured a trail width of $\approx 50^{\prime \prime}$.

The somewhat related brightness enhancements along some meteor streams, seen in the visible from the satellite D2A-Tournesol, have not been confirmed, neither by the photometric experiment on the Helios space probes (Richter et al. 1982) nor from IRAS. They probably are fainter than originally thought and certainly of lower surface brightness in the infrared than cometary trails or asteroidal bands.

The resonant dust ring outside the Earth's orbit

A leading/trailing asymmetry, with the zodiacal light at elongation $90^{\circ}$ being brighter in the trailing 
Table 22. Photometry of cometary trails

\begin{tabular}{lccccccc}
\hline \multicolumn{1}{c}{ Comet } & $R(\mathrm{AU})$ & $\Delta(\mathrm{AU})$ & $\Delta \Theta\left(^{\circ}\right)^{a)}$ & $\begin{array}{c}F_{\nu}(12 \mu \mathrm{m}) \\
(\mathrm{MJy} / \mathrm{sr})\end{array}$ & $\begin{array}{c}F_{\nu}(25 \mu \mathrm{m}) \\
(\mathrm{MJy} / \mathrm{sr})\end{array}$ & $\begin{array}{c}F_{\nu}(60 \mu \mathrm{m}) \\
(\mathrm{MJy} / \mathrm{sr})\end{array}$ & $\begin{array}{c}F_{\nu}(100 \mu \mathrm{m}) \\
(\mathrm{MJy} / \mathrm{sr})\end{array}$ \\
\hline Encke & 3.926 & 3.779 & 52.8 & - & $0.07 \pm 0.01$ & $0.06 \pm 0.01$ & - \\
Gunn & 2.681 & 2.473 & 0.82 & $0.22 \pm 0.06$ & $0.97 \pm 0.08$ & $0.55 \pm 0.03$ & - \\
Kopff & 1.577 & 0.953 & 0.53 & $1.04 \pm 0.14$ & $1.19 \pm 0.20$ & - & - \\
S-W 1 & 6.287 & 6.281 & 0.96 & - & $0.11 \pm 0.02$ & $0.15 \pm 0.02$ & $0.10 \pm 0.02$ \\
Tempel 2 & 1.460 & 1.149 & 0.37 & $2.44 \pm 0.09$ & $3.93 \pm 0.14$ & $1.54 \pm 0.035$ & - \\
\hline
\end{tabular}

a) $\Delta \Theta\left(^{\circ}\right)$ is angular distance behind comet in mean anomaly.

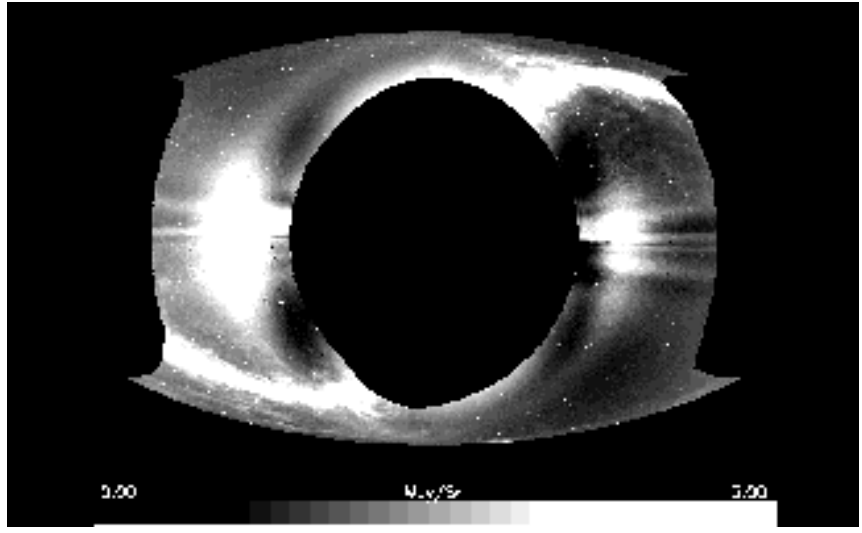

Fig. 54. Distribution of excess zodiacal light brightness due to the resonant dust ring outside the earth's orbit according to COBE measurements (Reach et al. 1995b). In this presentation, the position of the sun is at the center, the ecliptic runs horizontally through it, the ecliptic north pole is at top, the black central circle is the region inaccessible to COBE within $60^{\circ}$ elongation from the sun, and the two bright spots at $90^{\circ}$ from the sun on the ecliptic are at left the trailing (antapex) enhancement due to this dust ring, with a peak brightness of $1.7 \mathrm{MJy} / \mathrm{sr}$ at $25 \mu \mathrm{m}$, and at right the corresponding but weaker enhancement in leading (apex) direction.The S-shaped bright strip crossing the image is due to the Milky Way

(antapex) direction, has been found in the IRAS observations (Dermott et al. 1988, 1994) and has been confirmed by measurements of the DIRBE experiment on board the COBE spacecraft (Reach et al. 1995b). From the COBE measurements, the excess in the trailing direction in January 1990 was $0.05 \pm 0.01 \mathrm{MJy} / \mathrm{sr}$ or $4.8 \pm 1.0 \%$ at $4.9 \mu \mathrm{m}, 1.1 \pm 0.2 \mathrm{MJy} / \mathrm{sr}$ or $2.8 \pm 0.5 \%$ at $12 \mu \mathrm{m}$ and $1.7 \pm 0.1 \mathrm{MJy} / \mathrm{sr}$ or $2.4 \pm 0.15 \%$ at $25 \mu \mathrm{m}$. The region of enhanced brightness in the trailing direction is at $\approx 90^{\circ}$ from the sun, extending $30^{\circ}$ (FWHM) in latitude and $15^{\circ}$ (FWHM) in longitude (see Fig. 54, taken from Reach et al. 1995b). In the leading direction there is a smaller enhancement around elongation $80^{\circ}$.
These are quite extended structures (see Fig. 54). They are explained by resonant interaction of the orbiting earth with interplanetary particles drifting closer to the sun under the action of the Poynting-Robertson effect. This interaction leads to an inhomogeneous torus of enhanced dust density just outside the earth's orbit, with the earth sitting in a gap of this torus and the largest enhancement following it at a few tenths of an AU. The resonant ring structure therefore is expected to be a persistent feature of the zodiacal light.

\subsection{The zodiacal light seen from other places}

\subsubsection{Inside the solar system}

The decrease of zodiacal light brightness seen in a given viewing direction, occuring when the observer moves to larger heliocentric distances, has been measured along the ecliptic in the visual out to 3 AU (Pioneer 10, Toller \& Weinberg 1985) and can be reasonably predicted also for the infrared. The change to be expected when moving out of the ecliptic plane is less well known, but can be predicted from models fitting the out-of-ecliptic observations obtained from in-ecliptic positions at earth orbit.

For the infrared, Fig. 55 shows the predicted brightnesses in viewing directions parallel to the ecliptic and towards the ecliptic pole for an observer moving from 1 $\mathrm{AU}$ to $3 \mathrm{AU}$ in planes of different height above the ecliptic. The outward decrease is stronger for $12 \mu \mathrm{m}$ than for $25 \mu \mathrm{m}$. This is because the thermal emission of interplanetary dust is close to black-body radiation, and for blackbody radiation with decreasing temperature the shorter wavelengths first enter into the exponential decrease of the Wien part of the emission curve.

For the visual, Fig. 56 shows the corresponding decrease for the visual zodiacal light brightness when the observer moves from $1 \mathrm{AU}$ to $3 \mathrm{AU}$ in planes of different height above the ecliptic. Only one curve is shown, since any colour dependence is expected to be small.

The careful reader will note that the visual in-ecliptic brightness decreases a little slower with increasing distance than given in Sect. 8.2. This is because Giese (1979) 

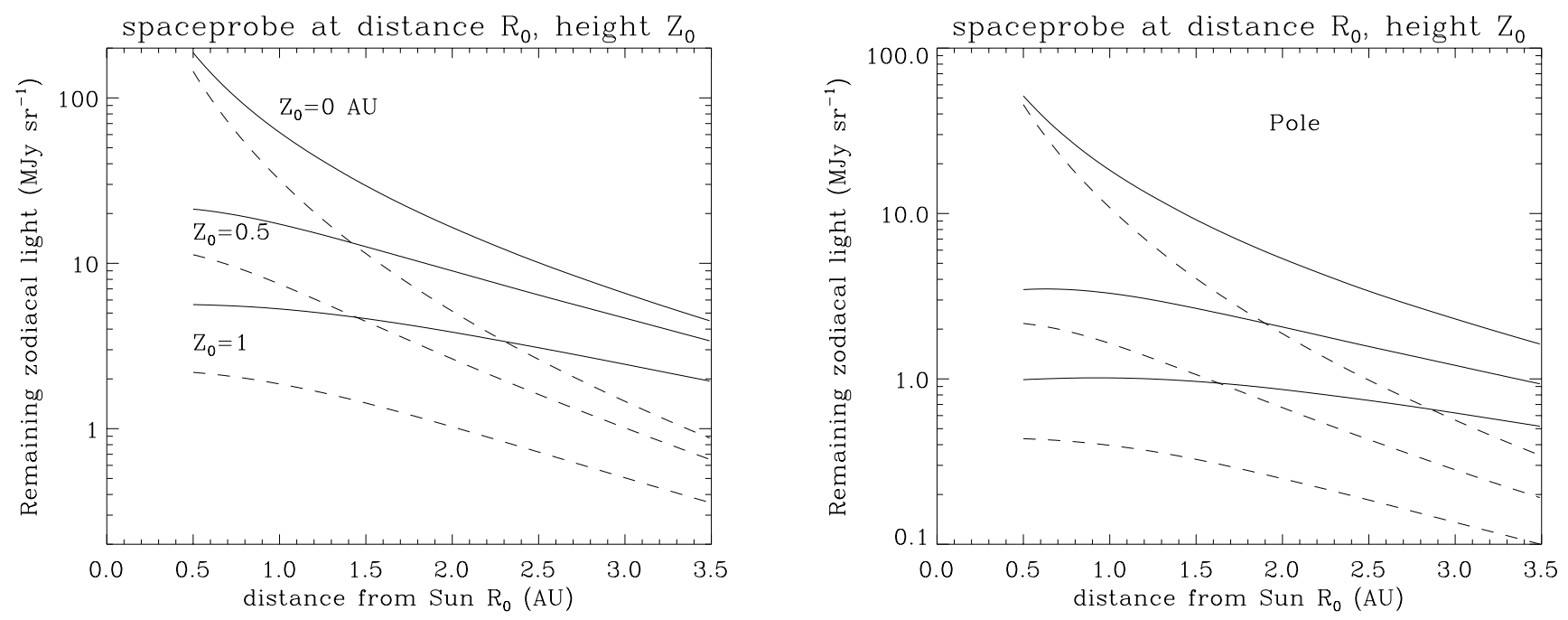

Fig. 55. Decrease of infrared zodiacal light brightness when moving out of the ecliptic plane. Left: for a viewing direction parallel to the ecliptic plane at elongation $\epsilon=90^{\circ}$. Right: for a viewing direction towards the ecliptic pole. The calculations have been done for a position of the observer in the ecliptic $\left(Z_{0}=0 \mathrm{AU}\right)$ and heights above of the ecliptic of $0.5 \mathrm{AU}$ and 1.0 $\mathrm{AU}$, as indicated in the figure. $R_{0}$ is the heliocentric distance of the observer, projected into the ecliptic plane. The solid and broken lines give the predicted run of brightness with heliocentric distance for a wavelength of $25 \mu \mathrm{m}$ and $12 \mu \mathrm{m}$, respectively. The calculations have assumed grey emission of the interplanetary particles, and radial decreases of spatial density $\sim r^{-1.4}$ and of particle temperature $\sim r^{-0.44}$ (W. Reach, private communication)

used a slightly different heliocentric radial brightness gradient, $I(\mathrm{R}) \sim R^{-2.2}$. The decrease as function of height above the ecliptic $Z_{0}$ is typical for the models of threedimensional dust distribution being discussed to explain the distribution of zodiacal light brightness (Giese et al. 1986). Since the three-dimensional dust distribution is not very well known, the decreases shown in Figs. 55 and 56 cannot be very accurate either.

\subsubsection{Surface brightness seen from outside the solar system}

Since the interplanetary dust cloud is optically very thin, the pole-on surface brightness at $1 \mathrm{AU}$ is just twice the polar surface brightness observed from the earth, and the edge-on surface brightness just twice the brightness observed at elongation $90^{\circ}$ in the ecliptic. The same type of relations hold for other heliocentric distances.

The brightness in an annulus extending over a range of heliocentric distances has to be obtained by integration. The total brightness as seen from outside very much depends on the distribution of interplanetary dust near the sun, and therefore is strongly model dependent. E.g., at least in the optical wavelength range an annulus of width $\mathrm{dr}[\mathrm{AU}]$ has a brightness $\sim r^{-1.3} \mathrm{dr}$ over a large region of the inner solar system, making the integrated brightness contribution strongly peaked towards the solar corona. In discussions of future planet-searching spacecraft (called DARWIN (Léger et al. 1996) and Terrestrial Planet Finder (Angel \& Woolf 1997)) a value of integrated zodiacal light brightness at $10 \mu \mathrm{m}$, when seen from a distance of $10 \mathrm{pc}$, of $70 \mu \mathrm{Jy}, 300$ to 400 times brighter than the Earth, is assumed (3.5 $10^{-5}$ of the solar brightness).

\section{Coronal brightness and polarisation}

\subsection{Overview}

The brightness of the corona surrounding the solar disk is composed of three main components: i) Thomsonscattered light from free electrons in the solar environment (K-corona) which is highly variable in space and time, ii) emission from coronal ions, especially in highly ionised states, and iii) contributions due to interplanetary dust (F-corona): solar radiation scattered on the dust particles in the visual, as well as thermal emission of these dust particles in the near and middle infrared regime. The F-corona dominates the visible coronal brightness from about $3 R_{\odot}$ distance from the center of the Sun outward and has an increasing contribution to the total coronal brightness at longer wavelengths (see Fig. 57).

For measurements in the corona, the elongation $\epsilon$ is often expressed in units of $R_{\odot}$, i.e. in terms of the minimum projected distance $r$ of the line of sight from the center of the Sun. Because the solar radius is $R_{\odot}=1 \mathrm{AU} / 214.94$ (Allen 1985), $1^{\circ}$ in elongation corresponds to $3.75 R_{\odot}$ (and $1 R_{\odot}$ to $16.0^{\prime}$ ), while more generally for an observer at the earth

$$
\sin \epsilon=r\left[R_{\odot}\right] \cdot \frac{1 R_{\odot}}{1 \mathrm{AU}} .
$$



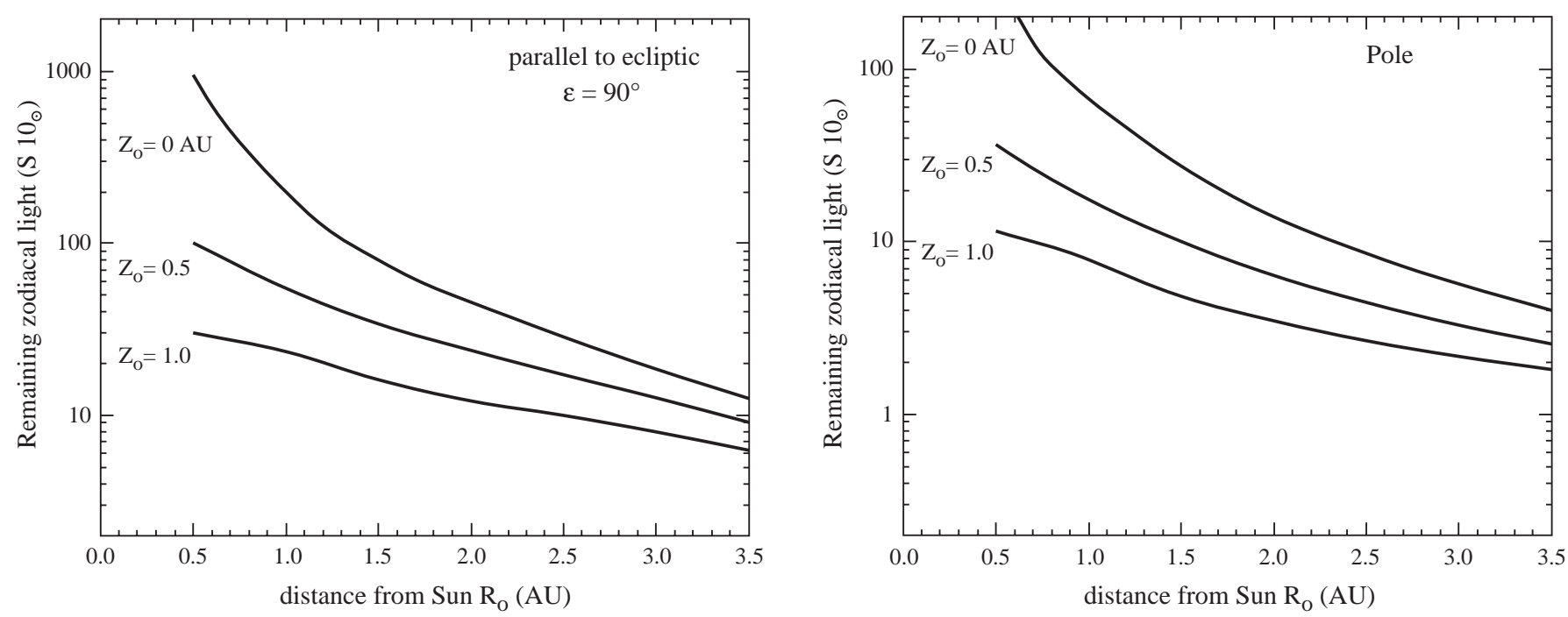

Fig. 56. Decrease of the visual brightness of the zodiacal light when the observer moves out of the ecliptic. Left: for a viewing direction parallel to the ecliptic plane at elongation $\epsilon=90^{\circ}$. Right: for a viewing direction towards the ecliptic pole. The curves show how the brightness changes with projected heliocentric distance $R_{0}$ (measured in the ecliptic) for different heights $Z_{0}$ above the ecliptic plane (interpolated from Giese 1979)

As mentioned in Sect. 2, coronal brightnesses often are expressed in terms of the average brightness of the solar disk as $B / B_{\odot}$, where $1 B / B_{\odot}=2.2210^{15} S 10_{\odot}=$ $1.4710^{4} F_{\odot} / \mathrm{sr}$.

\subsection{K-corona separation}

The main uncertainty in determination of the inner Fcorona is the separation from the K-coronal brightness. A common method of separation is based on the assumption that the F-coronal brightness is produced by diffraction of dust near the observer and hence unpolarized. This approach may be suitable for distances, respectively elongations of $<5 R_{\odot}$, the increasing polarization of the $\mathrm{F}$ corona at larger elongations (Blackwell et al. 1967) however leads to errors of this subtraction method. A further method of K-coronal separation uses the depth of Fraunhoferlines in the Solar spectrum. Both methods are described in Blackwell et al. (1967).

\subsection{Atmospheric and instrumental stray light}

Ground-based coronal observations generally are made during solar eclipses, with the local sky brightness constituting the main disturbance to be corrected for. The eclipse sky background on the ground may vary considerably with daily conditions as well as eclipse site. An early work by Blackwell et al. (1967) cites values of (1.819) $10^{-10} B_{\odot}$ for the eclipse sky background in the visible light, i.e. at wavelengths from 500 to $830 \mathrm{~nm}$. For $2.12 \mu \mathrm{m}$, MacQueen \& Greeley (1995) report a value of $10^{-10} B_{\odot}$ during the 1991 eclipse sky from Hawaii. However, these measurements suffered from thin clouds and the presence of high altitude aerosols from the Pinatubo eruption. The enhanced circumsolar sky brightness caused by diffraction on aerosols is called solar aureole. It may vary with elongation, and may be described as a function $A(r)$. Dürst (1982) derives values of about $10^{-11}$ to $10^{-9} B_{\odot}$ and a radial gradient according to $r^{-1.37}$ at $600 \mathrm{~nm}$ wavelength. Infrared results differ at the 1991 eclipse, but MacQueen \& Greeley (1995) find a description $A(r) \sim r^{-1.54}$ for the region from 3 to $9 R_{\odot}$ and a constant value of $210^{-8} \mathrm{~W}$ $\mathrm{cm}^{-2} \mu \mathrm{m}^{-1} \mathrm{sr}^{-1}$ (i.e. $10^{-10} B_{\odot}$ ) beyond for the infrared aureole during the 1991 eclipse. Instrumental straylight for externally occulted systems on satellites presently achieve straylight levels in the $10^{-10}$ to $10^{-12} B_{\odot}$ range and hence enable coronal observations out to at least $30 R_{\odot}$ (Brückner et al. 1995).

The values of polarization in the eclipse sky background range from $7.5 \%$ to $30 \%$ for ground based observations.

\subsection{Visual brightness}

Observations of the F-corona brightness (Fig. 58) are made during solar eclipses from ground, from rockets and from balloons in the visible and near infrared regime. Data were taken as well from space borne coronagraphs.

An early review of the visible coronal observations was given by Blackwell et al. (1967). They gave a description of the F-corona data as the continuation of the zodiacal light. A more recent review was given by Koutchmy \& Lamy (1985) including already infrared observations. They describe the visible F-corona brightness at wavelength $400 \mathrm{~nm}<\lambda<600 \mathrm{~nm}$ as proportional to $r^{-2.25}$ 


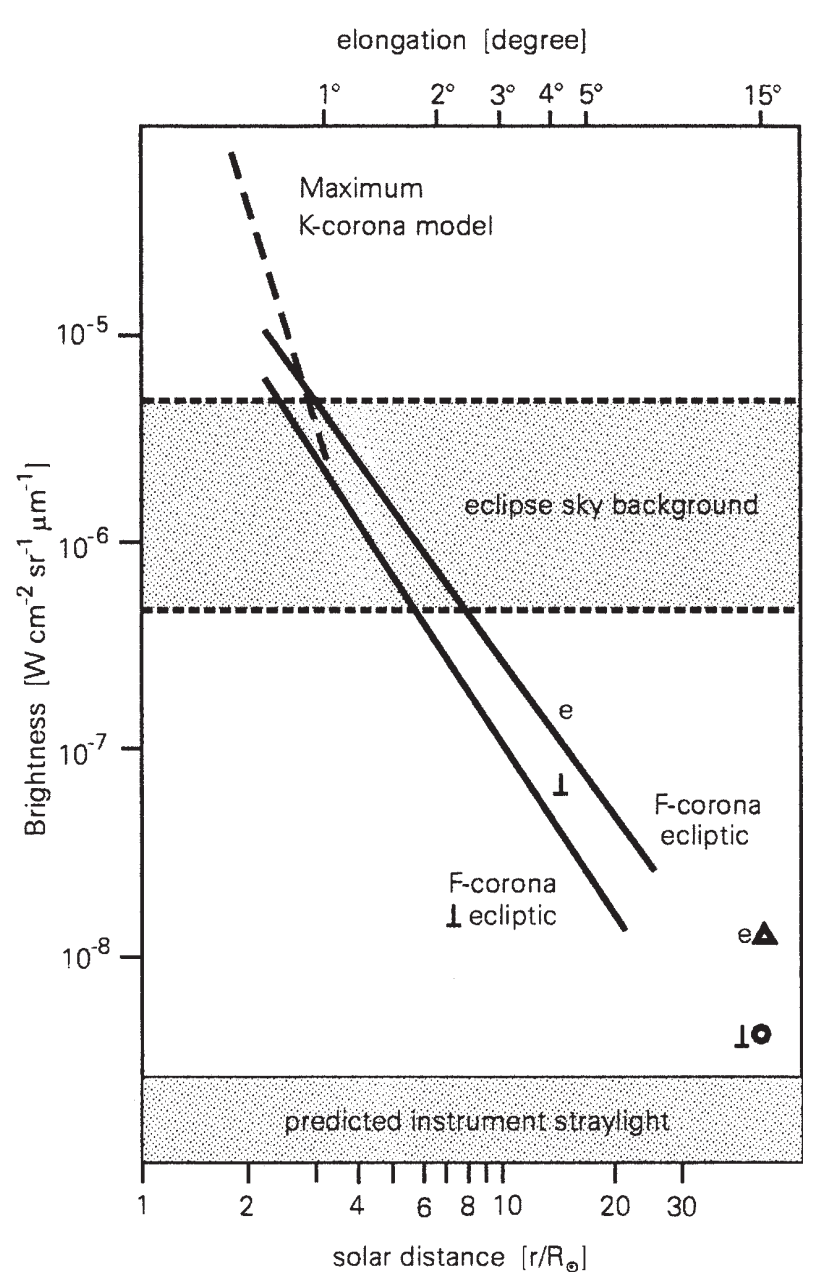

Fig. 57. The visible equatorial and polar F-corona brightnesses in comparison to typical values for K-corona, the aureole (circumsolar sky brightness enhancement) and instrumental straylight levels. At $15^{\circ}$, the brightnesses measured in the zodiacal light (see Sect. 8) are included

at the equator and $r^{-2.47}$ at the solar poles, based on a continuation of the zodiacal light data.

A measurement of the 1980 eclipse (Dürst 1982) yields a radial slope proportional to $r^{-2.44}$ in the equator and $r^{-2.76}$ at the poles when only fitting the slope to the eclipse observations in the range from 2 to $10 R_{\odot}$. Observations by Michard (1954) of the 1952 eclipse are fairly close to the model corona suggested by Koutchmy \& Lamy (1985), whereas the Blackwell data and the more recent observations by Dürst are a little lower. Observations from the Apollo 16 spacecraft describe the equatorial brightness beyond $20 R_{\odot}$ as $\sim r^{-1.93}$ (MacQueen et al. 1973).

We suggest to use for the visual spectral region a radial slope of the brightness as $r^{-2.5}$ in the equator and $r^{-2.8}$ at the pole (see Table 23). This takes the recent measurements into account as well as the fact that the scattering properties change due to the increasing diffraction peak at small scattering angles.

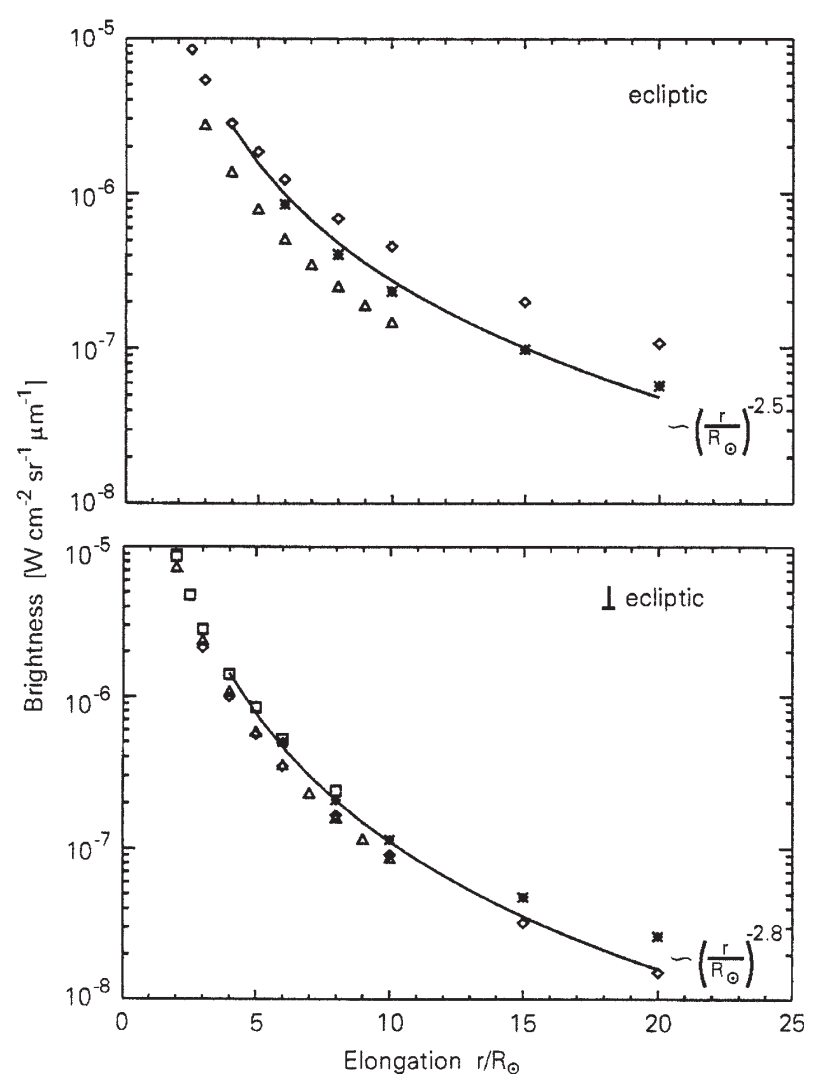

Fig. 58. The visible F-corona brightness, as measured along the ecliptic and the polar meridian. Asterisks: Blackwell (1995) (1954 eclipse); diamonds: Michard et al. (1954) (1952 eclipse); triangles Dürst (1982); squares Maihara et al. (1985). The power laws best representing these data are shown as solid lines

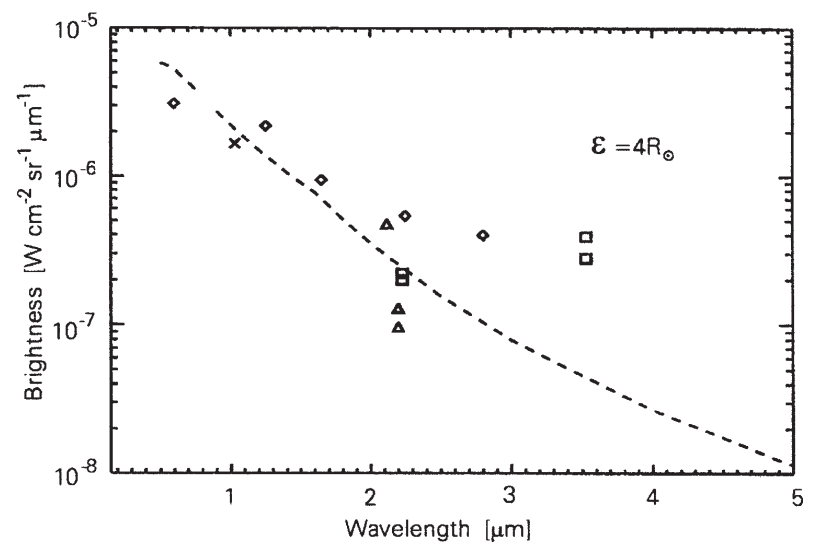

Fig. 59. The equatorial F-corona brightness at $4 R_{\odot}$. Diamonds: Maihara et al. (1985), cross: Smartt (1973), triangles: MacQueen (1968) (lower values), MacQueen \& Greeley (1995) (upper value); squares: Peterson (1967). The dashed line gives the solar spectrum normalized to wavelength $0.55 \mu \mathrm{m}$ 
Table 23. Proposed approximations to the F-coronal brightness distribution

\begin{tabular}{cccc}
\hline $\begin{array}{c}\langle\lambda\rangle \\
(\mu \mathrm{m})\end{array}$ & region & $\begin{array}{c}I(\lambda) \text { at } 4 R_{\odot} \\
\left(\mathrm{W} / \mathrm{m}^{2} \mathrm{sr} \mu \mathrm{m}\right)\end{array}$ & radial slope \\
\hline \multirow{2}{*}{0.5} & equatorial & $2.810^{-2}$ & $r^{-2.5}$ \\
& polar & $1.810^{-2}$ & $r^{-2.8}$ \\
2.12 & equatorial & $\approx 510^{-3}$ & $r^{-1.9}$ \\
& polar & $\approx 410^{-3}$ & $r^{-2.3}$ \\
\hline
\end{tabular}

For comparison: at $500 \mathrm{~nm}, 110^{-9} B / B_{\odot}=2.8410^{-2}$ $\mathrm{W} / \mathrm{m}^{2} \operatorname{sr} \mu \mathrm{m}$.

\subsection{Polarization and colour}

Due to the difficulties of K-corona separation, mentioned above, the polarization of the F-corona brightness is not fully understood so far. Figure 60 describes some data of the polarization of the total visible F-coronal brightness together with two models of F-corona polarization. The

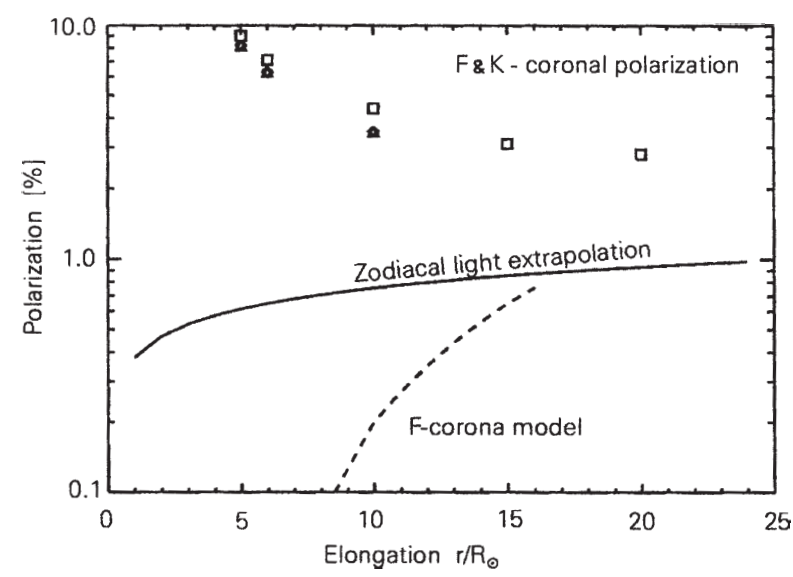

Fig. 60. The polarization of the total coronal brightness compared to the extrapolated zodiacal light model and the F-corona polarization according to Blackwell et al. (1967)

first case is the polarization curve extrapolated from the Zodiacal light polarization according to Eq. (16), the second case is the polarization derived by Blackwell et al. (1967). The classical coronal model suggested in Blackwell et al. gives almost no F-corona polarization within $10 R_{\odot}$. Furthermore, it has been suggested that an irregular slope of the F-corona polarization could either result from the beginning of the dust free zone around the Sun or reflect the existence of a dust ring. Observations of the 1991 eclipse show no hump in the polarization between 3 and $6.4 R_{\odot}$ and give an upper limit of $10 \%$ for the polarization.

Similar to the uncertainties in the determination of absolute brightness levels, the colour of the coronal bright- ness is not well defined (see Fig. 59). Since both, thermal emission of dust as well as a spectral change of scattering properties cause a reddening of the F-corona (Mann 1993), we can expect reddening to vary within the corona. As far as the visible F-corona is concerned, several estimates of the colour are either describing only the inner corona or may be biased from uncertain calibrations. However it seems to be proven that the reddening is stronger than in the Zodiacal light and is also stronger than the reddening of the inner Zodiacal light extrapolated to smaller elongations (Koutchmy \& Lamy 1985).

\subsection{Infrared}

\subsubsection{Near-infrared brightness}

Different values of the F-corona brightness at $4 R_{\odot}$ in the near infrared are shown in Fig. 59 in comparison to the solar spectral slope from Allen (1985), normalized to the F-coronal brightness at $0.5 \mu \mathrm{m}$. Although the differences between data sets are still large, the majority of data at longer wavelengths is above the extrapolated solar spectrum, indicating a contribution from the thermal emission of dust near the Sun.

Only the early infrared observations do not follow this trend.

The radial slope of the near infrared F-corona brightness can be derived from observations of the 1991 eclipse (Hodapp et al. 1992; Kuhn et al. 1994; MacQueen et al. 1994), however the sky conditions were mediocre, as mentioned above, and no accurate photometry was possible. The equatorial brightness was described as $B \sim r^{-1.9}$ and the polar brightness as $B \sim r^{-2.3}$, for regions inside $8 R_{\odot}$. Observations of the 1973 eclipse by Smartt (1973) in the near-infrared $(\lambda=1.03 \mu \mathrm{m})$ show a similar radial slope of $r^{-1.9}$ between $3 R_{\odot}$ and $5 R_{\odot}$ and of $r^{-2.2}$ in the outer corona.

\subsubsection{Mid-infrared brightness}

An important constituent of interplanetary dust particles is silicate, which exhibits a pronounced reststrahlen band in the $10 \mu \mathrm{m}$ wavelength region. An enhanced brightness of the mid infrared corona could reveal for instance the presence of small silicate particles near the Sun (cf. Kaiser 1970). Unfortunately, data in the mid infrared regime are biased, either by scattered light components from a window in case of aircraft measurements (Lena et al. 1974), or by strong atmospheric emission and fluctuations in the case of observations from ground (Mankin et al. 1974).

\subsubsection{IR - humps and dust rings}

The first measurement of the near infrared coronal brightness showed a deviation of the slope from a continuous increase within the corona, with brightness enhancements by a factor of $3-3.5$. Several of these humps were 
seen by Peterson (1967) and MacQueen (1968), and later checked by Isobe et al. (1985), Mizutani et al. (1984), and Tollestrup et al. (1994). Model calculations by Mukai \& Yamamoto (1979) showed that these humps could be explained by a dynamical effect that produces dust rings around the Sun. It is also possible that a hump of the infrared brightness is produced when the line of sight crosses the beginning of a dust free zone (Mann 1992). A model calculation by Kimura et al. (1997) shows that this effect may depend on the material composition of dust near the Sun. However, there have been several unpublished observations which could not detect a dust ring (see Isobe 1993), and observers of the 1991 eclipse could not confirm the existence of humps in the near infrared brightness (Hodapp et al. 1992; Kuhn et al. 1994; Tollestrup et al. 1994). In this context we should mention that the presently available data do not allow for a study of temporal effects in the F-coronal brightness, such as the appearance of dust clouds from sun-grazing comets or temporal dust rings.

\section{Integrated starlight}

\subsection{Model predictions based on star counts}

The combined light from unresolved stars contributes to the sky brightness from the ultraviolet through the midinfrared, with the contribution being dominated by hot stars and white dwarfs at the shortest wavelengths, main sequence stars at visual wavelengths, and red giants in the infrared (Mathis et al. 1983). The integrated starlight contribution to the sky brightness depends on the ability of an experiment to resolve out the brightest stars, which in turn depends on the Galactic latitude. If we suppose that stars brighter than flux $F_{0}$ are resolved and excluded from the diffuse sky brightness, then the integrated starlight contribution is the integral over the line of sight of the brightness contributions from stars fainter than $F_{0}$,

$$
I_{\mathrm{ISL}}=\int_{0}^{F_{0}} \mathrm{~d} F \frac{\mathrm{d} N(l, b)}{\mathrm{d} F} F,
$$

where $\frac{\mathrm{d} N(l, b)}{\mathrm{d} F} \mathrm{~d} F$ is the number of stars in the flux range $F$ to $F+\mathrm{d} F$, for a line of sight towards galactic coordinates $l, b$. In principle, we must also integrate the counts over the beam and divide by the beam size, but in practice, the variation in the number of sources over a beam is often small except for large beams at low galactic latitude. (In those cases, Eq. (28) is replaced by

$$
I_{\mathrm{ISL}}=\frac{1}{\Omega_{\mathrm{b}}} \int_{\text {beam }} \mathrm{d} \Omega \int_{0}^{F_{0}} \mathrm{~d} F \frac{\mathrm{d} N(l, b)}{\mathrm{d} F} F,
$$

where $\Omega_{\mathrm{b}}$ is the beam solid angle.) The cumulative number of sources increases less steeply than $1 / F$ for the fainter stars, so that the integral converges; in the near-infrared at $2.2 \mu \mathrm{m}$, the peak contribution to the sky brightness occurs for stars in the range $0<K<6$. For reference,
$K=0$ corresponds to $F_{\nu}=670 \mathrm{Jy}$ (Campins et al. 1985), and there is of order 1 star per square degree brighter than $K=6$, and (extrapolating) there is one star per square arcminute brighter than $K=15$. Thus, for comparison, the DIRBE survey ( $42^{\prime}$ beam, $K=4$ detection limit) resolves about $50 \%$ of the starlight in the $K$ band, while the DENIS survey (limiting magnitude $K=14$ ) should resolve some $97 \%$. Similarly, in the far-ultraviolet, the FAUST survey resolves some $96 \%$ of starlight (Cohen et al. 1994). And at visible wavelengths, star counts near the North Galactic Pole (Bahcall \& Soneira 1984) also show that the visible surface brightness for low-resolution observations is strongly dominated by the brightest stars $(\approx 6-13 \mathrm{mag})$. It is for deep surveys with low angular resolution that we address the remainder of this discussion of integrated starlight.

Table 24. Surface brightness due to integrated starlight (given as $\lambda I_{\lambda}$, respectively $\nu I_{\nu}$ )

\begin{tabular}{ccc}
\hline wavelength $(\mu \mathrm{m})$ & \multicolumn{2}{c}{ surface brightness $\left(10^{-9} \mathrm{~W} \mathrm{~m}^{-2} \mathrm{sr}^{-1}\right)$} \\
\cline { 2 - 3 } & $b=30^{\circ}$ & North Gal. Pole \\
\hline 0.1565 & 62 & 24 \\
0.55 & 577 & 250 \\
2.2 & 205 & 105 \\
12 & 6.1 & 3.0 \\
\hline
\end{tabular}

To estimate the contribution of integrated starlight to a deep observation, one must sum the contribution from each type of star along the line of sight. One may recast the integral in Eq. (28) more intuitively by integrating over the line of sight for each class of object (which has a fixed luminosity):

$$
I_{\mathrm{ISL}}=\sum_{i} \int_{s_{i}}^{\infty} \mathrm{d} s s^{2} n_{i}(s) \frac{L_{i}}{4 \pi s^{2}}
$$

where $n_{i}$ is the number density and $L_{i}$ the luminosity of sources of type $i$. The integral extends outward from a given inner cutoff $s_{i}$ that depends on the source type through $s_{i}^{2}=L_{i} / 4 \pi F_{0}$. Bahcall \& Soneira $(1980,1984)$ constructed such a model, with the Galaxy consisting of an exponential disk and a power-law, spheroidal bulge. The shape parameters (vertical scale height and radial scale length of the disk, and bulge-to-disk density ratio) of the Galactic star distribution were optimised to match the star counts. A more detailed model (SKY), both in terms of Galactic shape and the list of sources, has been constructed by M. Cohen and collaborators (Wainscoat et al. 1992; Cohen 1993, 1994; Cohen et al. 1994; Cohen 1995).

Examples of the surface brightness predicted by the SKY model for two lines of sight and four wavebands, from 


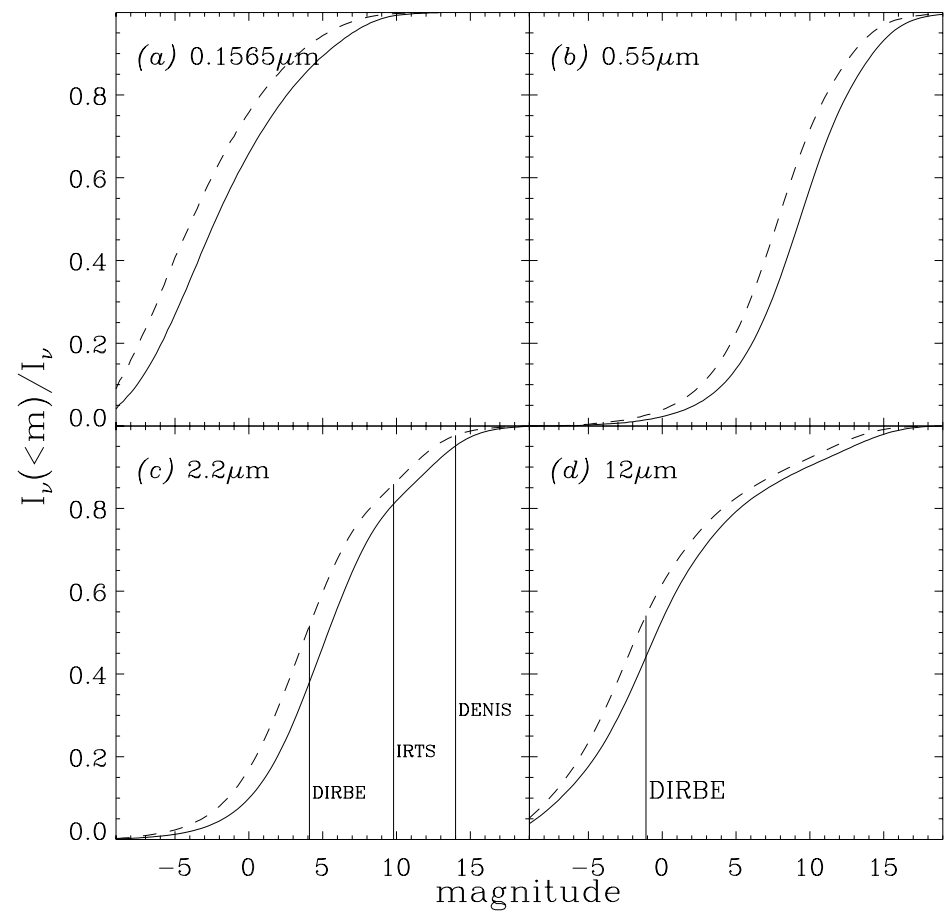

Fig. 61. Fraction of integrated starlight due to stars brighter than a given magnitude, for two lines of sight: the NGP (dashed curves) and a region at $30^{\circ}$ galactic latitude (solid curves). Each panel is for a different wavelength: a) $1565 \AA$, b) $5500 \AA$, c) $2.2 \mu \mathrm{m}$, and d) $12 \mu \mathrm{m}$. In panel c), the vertical lines indicate the magnitude limits adopted in analysis of DIRBE (Arendt et al. 1997), IRTS (Matsumoto et al. 1997), and DENIS (Epchtein 1994, 1997) observations are shown

the ultraviolet to the mid-infrared, are shown in Fig. 61. Of these, the basis for the ultraviolet part is discussed in more detail in Sect. 10.2.2 below. Each curve in Fig. 61 gives the fractional contribution to the surface brightness due to stars brighter than a given magnitude. The total surface brightness for each wavelength and line of sight is given in Table 24. The sky brightness due to unresolved starlight can be estimated for any experiment given the magnitude limit to which it can resolve stars. First, determine the fraction, $f$, of brightness due to stars brighter than the limit using Fig. 61 . Then, using the total brightness of starlight, $I_{\text {ISL }}$ from Table 24, the surface brightness due to unresolved stars is $I_{\text {ISL }} \times(1-f)$. - For specific results of the SKY model contact Martin Cohen directly.

The old compilations of integrated starlight in the visual by Roach \& Megill (1961) and Sharov \& Lipaeva (1973) do not have have high $\left(\approx 1^{\circ}\right)$ spatial resolution and are not calibrated to better than $\approx 15 \%$. However, they still give useful information, are conveniently available in tabulated form, and have been used, e.g. in work to be discussed below in Sects. 11.2 and 12.2.1.

\subsection{Ultraviolet}

\subsubsection{Near ultraviolet $(180 \mathrm{~nm}-300 \mathrm{~nm})$}

The UV astronomy experiment S2/68 (Boksenberg et al. 1973) provided catalogs of stellar UV brightness over the sky in one photometric channel at $274 \mathrm{~nm}(\Delta \lambda=30 \mathrm{~nm})$ and three spectroscopic channels around $156.5 \mathrm{~nm}(\Delta \lambda=$ $33 \mathrm{~nm}), 196.5 \mathrm{~nm}(\Delta \lambda=33 \mathrm{~nm})$, and $236.5 \mathrm{~nm}(\Delta \lambda=$ $33 \mathrm{~nm}$ ). Gondhalekar (1990) integrated over the spectroscopic channels to provide photometric information at all of the four UV wavelengths. The photometric accuracy is $\approx 10 \%$. Only the 47039 stars with UV flux larger than $1.0 \times 10^{-12} \mathrm{erg} \mathrm{cm}^{-1} \mathrm{~s}^{-1} \mathrm{sr}^{-1} \AA^{-1}\left(m_{\mathrm{UV}} \approx 8 \mathrm{mag}\right)$ in at least one of the four passbands were kept for calculating the integrated starlight brightness over the sky. The resulting brightnesses are given in Tables 25 to 28 .

Brosch (1991) also attempted to produce a galaxy model for the UV. He adapted the Bahcall \& Soneira (1980) galaxy model by suitable colour relations to the $150-250 \mathrm{~nm}$ sky, and added Gould's belt and white dwarfs. He compared in his Fig. 3 the model with the limited results available from a wide field UV imager flown on Apollo 16 (Page et al. 1982) and found reasonable agreement between his model and these data, 
Table 25. The intensity of stellar UV radiation at $156.5 \mathrm{~nm}$ in bins of $10^{\circ} \times 10^{\circ}$ in units of $10^{-10} \mathrm{~W} \mathrm{~m}^{-2} \mathrm{sr}^{-1} \mu \mathrm{m}^{-1}, \mathrm{respectively}$ $10^{-11} \mathrm{erg} \mathrm{cm}^{-1} \mathrm{~s}^{-1} \mathrm{sr}^{-1} \AA^{-1}$. The limits of the bins are given in degrees of galactic logitude and galactic latitude with the table. Only stars brighter than a certain flux limit (see text) were included. From Gondhalekar (1990)
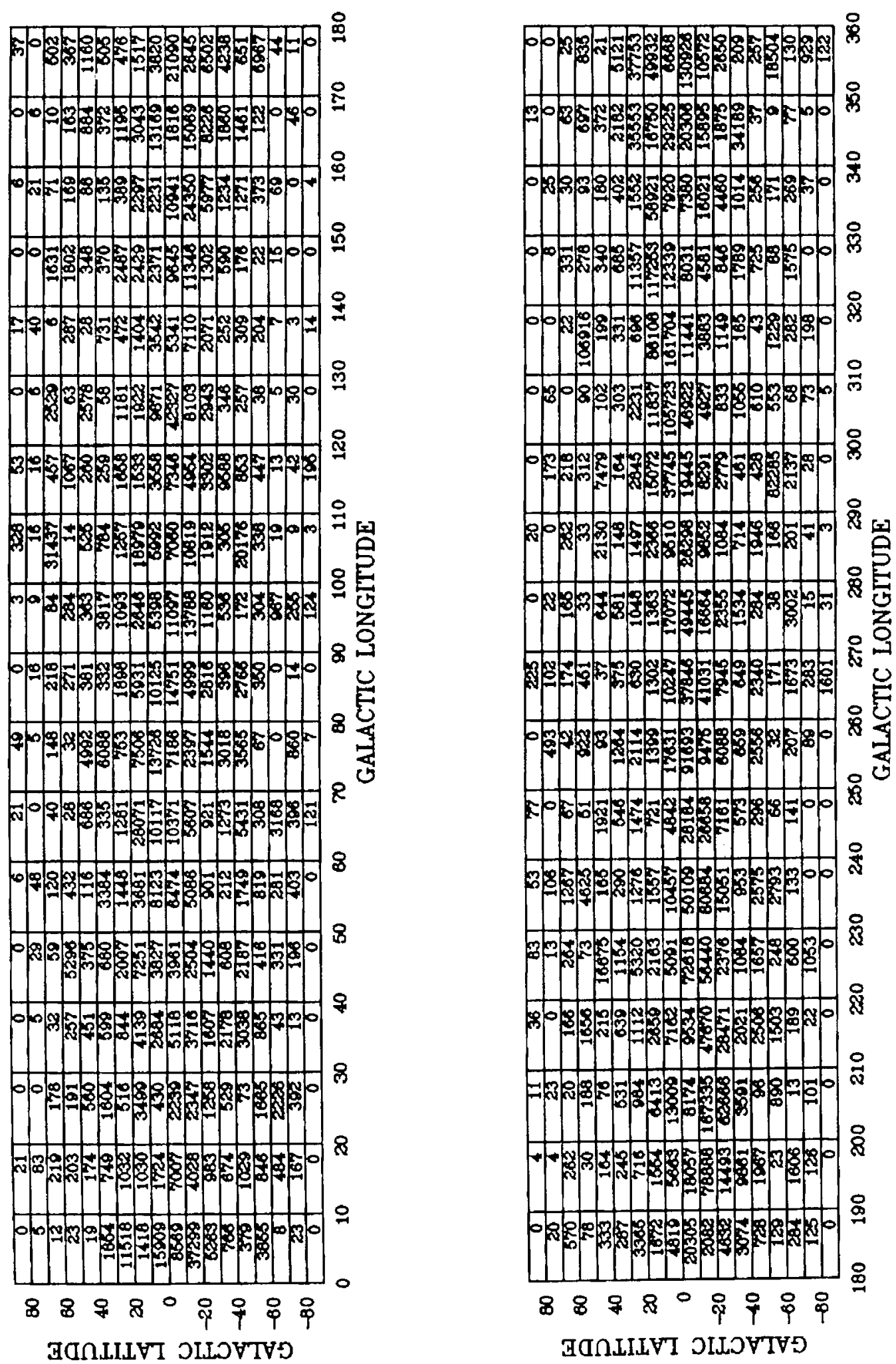
Table 26. The intensity of stellar UV radiation at $196.5 \mathrm{~nm}$ in bins of $10^{\circ} \times 10^{\circ}$ in units of $10^{-10} \mathrm{~W} \mathrm{~m}^{-2} \mathrm{sr}^{-1} \mu \mathrm{m}^{-1}, \mathrm{respectively}$ $10^{-11} \mathrm{erg} \mathrm{cm}^{-1} \mathrm{~s}^{-1} \mathrm{sr}^{-1} \AA^{-1}$. The limits of the bins are given in degrees of galactic logitude and galactic latitude with the table. Only stars brighter than a certain flux limit (see text) were included. From Gondhalekar (1990)
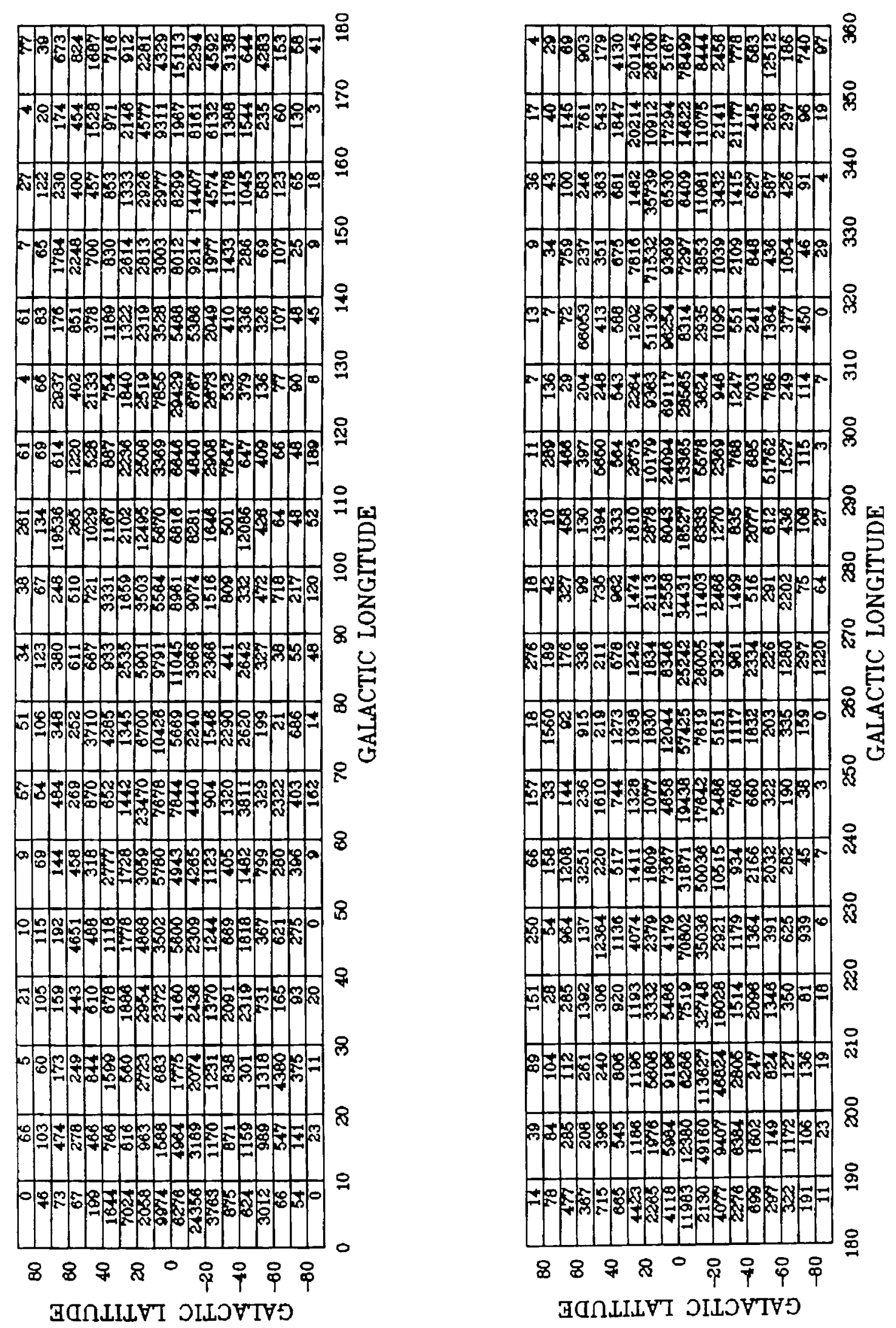
Table 27. The intensity of stellar UV radiation at $236.5 \mathrm{~nm}$ in bins of $10^{\circ} \times 10^{\circ}$ in units of $10^{-10} \mathrm{~W} \mathrm{~m}^{-2} \mathrm{sr}^{-1} \mu \mathrm{m}^{-1}, \mathrm{respectively}$ $10^{-11} \mathrm{erg} \mathrm{cm}^{-1} \mathrm{~s}^{-1} \mathrm{sr}^{-1} \AA^{-1}$. The limits of the bins are given in degrees of galactic logitude and galactic latitude with the table. Only stars brighter than a certain flux limit (see text) were included. From Gondhalekar (1990)
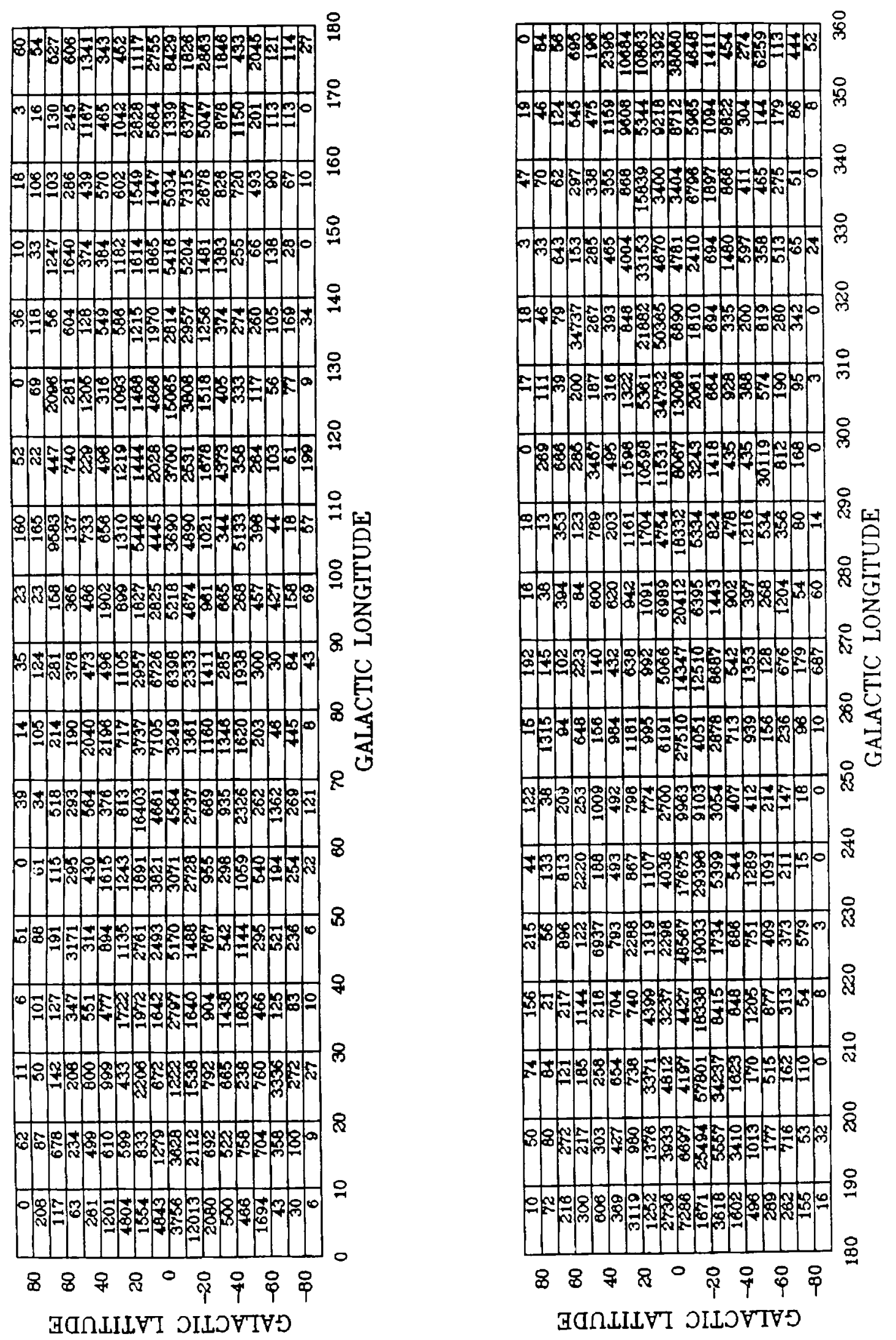
Table 28. The intensity of stellar UV radiation at $274 \mathrm{~nm}$ in bins of $10^{\circ} \times 10^{\circ}$ in units of $10^{-10} \mathrm{~W} \mathrm{~m}^{-2} \mathrm{sr}^{-1} \mu \mathrm{m}^{-1}, \mathrm{respectively}$ $10^{-11} \mathrm{erg} \mathrm{cm}^{-1} \mathrm{~s}^{-1} \mathrm{sr}^{-1} \AA^{-1}$. The limits of the bins are given in degrees of galactic logitude and galactic latitude with the table. Only stars brighter than a certain flux limit (see text) were included. From Gondhalekar (1990)
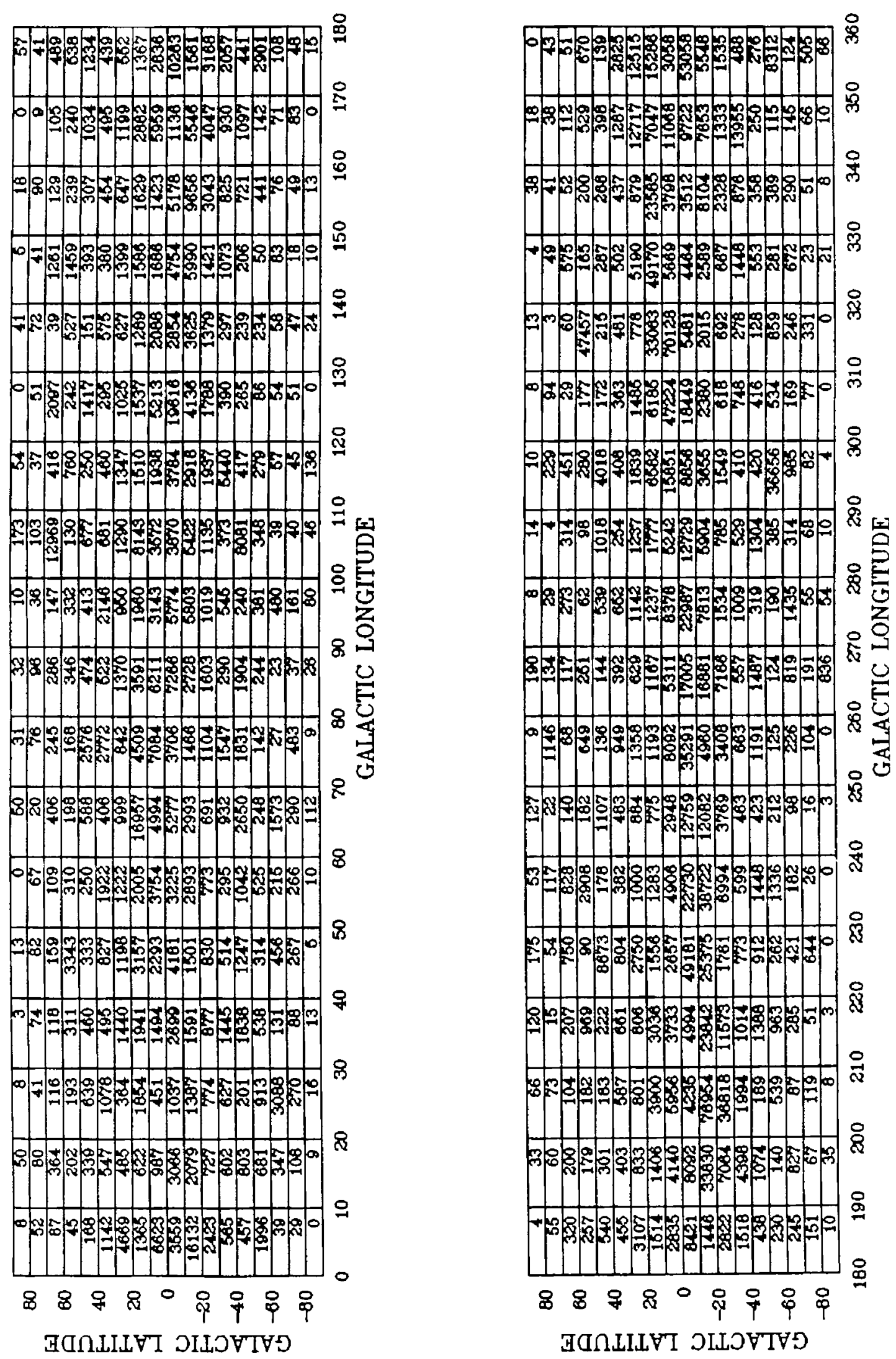

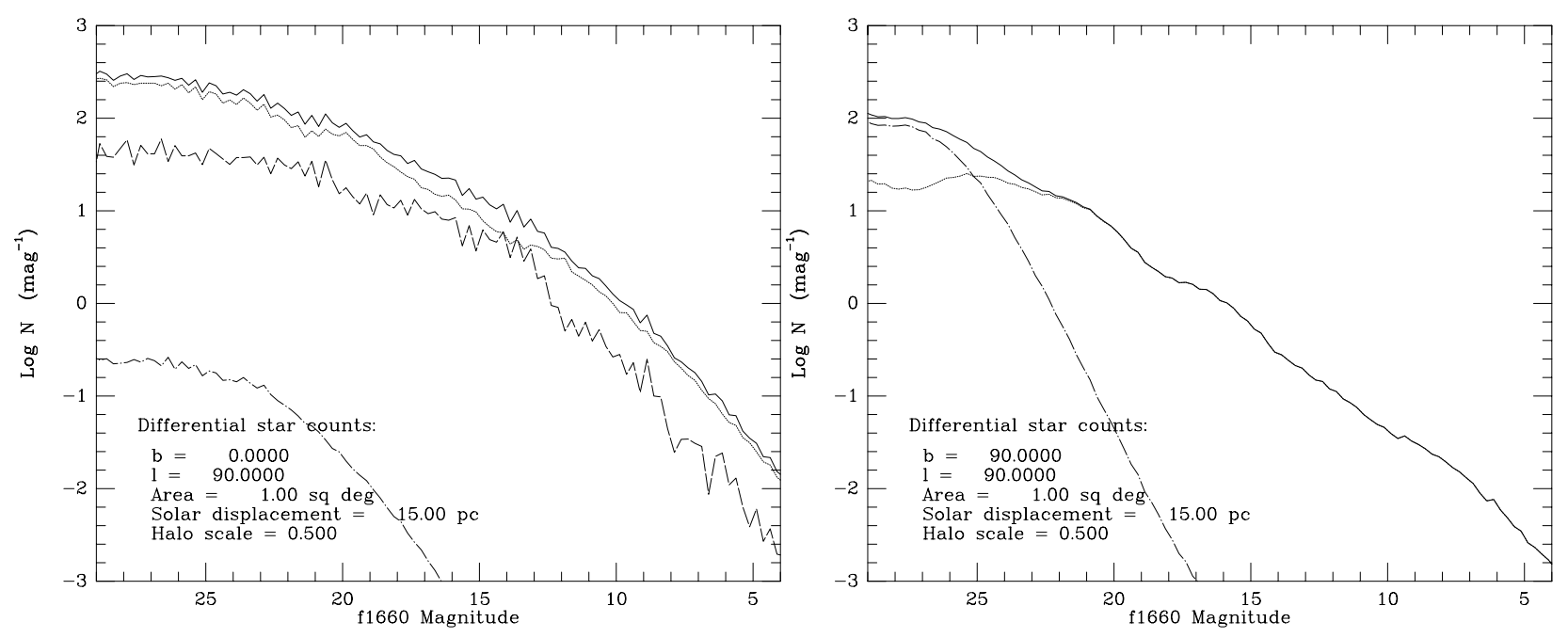

Fig. 62. Differential star counts as a function of FUV magnitude for a position in the galactic plane at $l=90^{\circ}$ (left) and for the galactic pole (right). Solid line: total contribution, faint dotted line: disk component, dash-dot line: halo contribution, long-dashed line: spiral arms plus local spur (shown only for the field in the galactic plane)

but otherwise does not give an explicit description of the model.

\subsubsection{FUV $(91.2 \mathrm{~nm}-180 \mathrm{~nm})$}

Table 25 discussed in the last subsection actually belongs to the FUV range.

As far as modelling is concerned, the stellar contributions to the FUV sky brightness have been well characterized. The optical and infrared SKY model of Cohen (1994) has been expanded into the FUV by fitting it to observations on the FUV sky obtained with the FAUST FUV telescope (Bowyer et al. 1993). The FAUST camera had obtained observational data on 5000 sources in 21 separate fields in the $140-180 \mathrm{~nm}$ bandpass. These data covered FUV magnitudes from 5 to 12 . The model resulting from the comparison to these data (Cohen et al. 1994) provides an excellent fit to the available FUV observations. The extrapolated flux for magnitudes greater than 12 is less than $4 \%$ of the total point source flux and is less than $1 \%$ of the FUV diffuse sky brightness.

As is the case for other wavelength bands, the integrated starlight in the FUV (and also the near ultraviolet) is concentrated toward the plane of the Galaxy. In Fig. 62 we display two examples of how the model accounts for the stellar contribution in the ultraviolet (kindly provided by Martin Cohen). The figure shows differential star counts as a function of a FUV magnitude centered at $166 \mathrm{~nm}$, both for a position in the galactic plane at $l=90^{\circ}$ and for the galactic pole. In both parts of the figure, the solid line is the total number of stars per square degree per magnitude interval, the disk component is shown by the faint dotted line, and the dash-dot line is the halo contribution. For the galactic plane (left diagram), the halo component is of lesser importance, but the spiral arms plus local spur
Table 29. Total integrated surface brightness in the range $140 \mathrm{~nm}-180 \mathrm{~nm}$ due to point sources, as given by the SKY model $^{a}$ at a galactic longitude of $90^{\circ}$ as predicted by the SKY model

\begin{tabular}{cccc}
\hline $\begin{array}{c}\text { Galactic } \\
\text { latitude }\end{array}$ & $\begin{array}{c}I_{\nu} \\
\mathrm{mJy} / \square^{\circ}\end{array}$ & $\begin{array}{c}I_{\lambda} \\
\mathrm{W} / \mathrm{m}^{2} \mathrm{sr} \mu \mathrm{m}\end{array}$ & $\begin{array}{c}I \\
\text { photons } / \mathrm{cm}^{2} \mathrm{~s} \mathrm{sr} \AA\end{array}$ \\
\hline $90^{\circ}$ & $26.6 \pm 0.01$ & $10210^{-10}$ & 82.5 \\
$80^{\circ}$ & $40.6 \pm 0.02$ & $15610^{-10}$ & 125.8 \\
$70^{\circ}$ & $49.7 \pm 0.03$ & $19110^{-10}$ & 153.8 \\
$60^{\circ}$ & $58.2 \pm 0.03$ & $22410^{-10}$ & 180.4 \\
$50^{\circ}$ & $70.9 \pm 0.05$ & $27310^{-10}$ & 219.5 \\
$40^{\circ}$ & $89.8 \pm 0.19$ & $34510^{-10}$ & 278.0 \\
$30^{\circ}$ & $122.1 \pm 0.3$ & $46910^{-10}$ & 378.2 \\
$20^{\circ}$ & $185.0 \pm 0.6$ & $70910^{-10}$ & 571.1 \\
$10^{\circ}$ & $483.0 \pm 12.9$ & $186010^{-10}$ & 1496 \\
$0^{\circ}$ & $429.7 \pm 7.8$ & $165010^{-10}$ & 1330 \\
\hline
\end{tabular}

${ }^{a}$ Since this model was primarily constructed for the infrared, it cannot be expected to be accurate in the ultraviolet at low galactic latitudes $\left(|b| \leq 10^{\circ}\right)$, where the effects of clumpiness of the interstellar medium get dominating (Caplan \& Grec 1979).

contribution have to be taken into account (long-dashed curve). Table 29 gives the total stellar surface brightness in the $140-180 \mathrm{~nm}$ band as a function of galactic latitude. The brightness varies with galactic longitude; in this case we show the values for $l=90^{\circ}$.

In an attempt to unify the above information on ultraviolet integrated starlight, at present we suggest to rely on Tables 25 to 28 for the absolute and total brightness level, and to use the models demonstrated above for purposes like extrapolation to the contribution of faint stars 
or breakdown of the total brightness into the contribution of different components or brightness intervals.

\subsection{Ground-based UBVR photometries}

Besides airglow and zodiacal light, the Milky Way is the third major contributor to the diffuse night sky brightness in the visual spectral domain. Its light is fixed with respect to an inertial system of reference and also is constant over large time scales. For absolute brightness determinations, space experiments, free of disturbance by the earth's atmosphere, are best suited, for studies of structures, ground-based surveys are preferable because of their greater flexibility. In any case one has to be aware of the presence of diffuse galactic light which on the average contributes between $20 \%$ and and $30 \%$ of the Milky Way brightness.

Efforts to describe the distribution of the Milky Way's brightness are numerous and can be traced back far into the past (Ptolemy's Almagest). Difficulties to get rid of atmospheric disturbances still are present in the classical paper by Elsässer and Haug in 1960, which otherwise, for the first time, presented photoelectric measurements of our Galaxy with a reasonable resolution in well defined passbands (see Tables 30 and 31).

The four photometries of the Southern Milky Way presented here in colour as Figs. $63-67$ profit from the now more effective correction for the atmospheric effects. They cover the whole range in longitudes and galactic latitudes from $-40^{\circ}$ to $+40^{\circ}$. They have a high angular resolution $(0.25 \times 0.25$ square degrees $)$. Moreover, all wavelength bands are processed in the same way, and so the colours $U-B, B-V, V-R$ should be quite coherent. The figures presented here only give an overview, although the linear scale of the colour bar will allow coarse interpolation. The data are accessible in digital form at the astronomical data center Centre de Données Stellaires (CDS) in Strasbourg under

http://cdsweb.u-strasbg.fr/htbin/myqcat3?VII/199/

It is planned to make accessible to the public under this address step by step all major ground-based photometries of the Milky Way contained in Table 30, in particular also the $B$ photometry by Classen (1976), which has the advantage of large sky coverage and which fits quite well to the Helios and Pioneer space probe data (see Fig. 70 below). For further information with respect to the four photometries discussed, see the papers by Kimeswenger et al. (1993) and Hoffmann et al. (1997). As an example for the kind of spatial detail to be expected, Fig. 67 shows on an enlarged scale the $U B V R$ photometry for the Coalsack region.
The $U B V R$ photometries shown in Figs. 63-67 are based on photographic exposures, calibrated in situ by photoelectric measurements of the night sky. The raw data were obtained in 1971 by Schlosser \& Schmidt-Kaler at La Silla (Schlosser 1972). The well known disadvantages of photographic plates (their relatively low inherent accuracy, for instance) do not count so much if one considers the often rapid variations of the night sky in total. Such changes especially affect scanning photometers and reduce their inherent accuracy. A posteriori, it is virtually impossible to discriminate between temporal and spatial variations. For Figs. 63- 67, a wide angle camera (FOV $135^{\circ}$ ) was employed, which integrated the night sky at the same time, thus avoiding the above mentioned unwanted effects. Tables 30-32 contain supporting information. Table 30 gives a synopsis of photometries in the visual and near-visual spectral spectral domain. This list contains only photometries covering the whole Galaxy or a major part of it (for more details, see Scheffler 1982). Some photometries of smaller galactic areas are contained in Table 31. In Table 32, the four Bochum photometries shown here are compared to those of other authors. Because the Helios data (Hanner et al. 1978; Leinert \& Richter 1981) are considered a well calibrated reference, these space probe measurements are also included here for comparison. The same is true for the south polar region subset of Pioneer data shown by Weinberg (1981) and the subset presented by Toller (1990), while a much more complete overview on the Pioneer measurements of integrated starlight will be given in the following subsection.

\subsection{Pioneer 10/11 spaceborne visual photometry}

Small imaging photopolarimeters (IPP's) on the Pioneer 10 and 11 deep space probes were used during cruise phases (between and beyond the planets) to periodically measure and map over the sky brightness and polarisation in blue $(395 \mathrm{~nm}-495 \mathrm{~nm})$ and red $(590 \mathrm{~nm}-690 \mathrm{~nm})$ bands. This was done at heliocentric distances beyond 1.015 AU (Weinberg et al. 1974; Hanner et al. 1974). Early results suggested that observations of the same sky regions decreased in brightness with heliocentric distance $R$ to $\approx 3.3 \mathrm{AU}$ (Weinberg et al. 1974; Hanner et al. 1976), beyond which there was no observable change; i.e., the zodiacal light became vanishingly small compared to the background galactic light (i.e. was less than $2 S 10_{\odot}$ ). Subsequent analysis (Schuerman et al. 1977) found this detectability limit to be $2.8 \mathrm{AU}$. Thus, for sky maps made between $1 \mathrm{AU}$ and $2.8 \mathrm{AU}$, the observations give the sum of zodiacal light and background starlight, while beyond 2.8 AU the background starlight, including some diffuse galactic light, could be observed directly. We summarise here those observations from beyond 2.8 AU.

Approximately 80 sky maps were obtained with the Pioneer 10 IPP, starting in March 1972, of which 50 maps fall into the year 1972 (see Table 33). The FOV's covered 
Fig. 63. $U$ photometry of the Southern Milky Way. The photometry is accompanied by a colour bar. Its left end corresponds to $-100 S_{10}$. The brightness at the right end of the bar is $450 S_{10}$ units $(U)$. The scale is linear. White areas denote non-valid data 
Fig. 64. $B$ photometry of the Southern Milky Way. The photometry is accompanied by a colour bar. Its left end corresponds to $-100 S_{10}$. The brightness at the right end of the bar is $550 S_{10}$ units $(B)$. The scale is linear. White areas denote non-valid data 
Fig. 65. $V$ photometry of the Southern Milky Way. The photometry is accompanied by a colour bar. Its left end corresponds to $-100 S_{10}$. The brightness at the right end of the bar is $900 S_{10}$ units $(V)$. The scale is linear. White areas denote non-valid data 
Fig. 66. $R$ photometry of the Southern Milky Way. The photometry is accompanied by a colour bar. Its left end corresponds to $-100 S_{10}$. The brightness at the right end of the bar is $2600 S_{10}$ units $(R)$. The scale is linear. White areas denote non-valid data 
Fig. 67. Synopsis of the Carina-Coalsack region in $U, B, V, R$. To facilitate comparison, the levels are adjusted for an optimal visualization. The $S_{10}$-isophotes in the sense of "outer broken line, continuous line and inner broken line" are (150, 250, 380) for $U,(150,230,400)$ for $B,(250,500,800)$ for $V$, and $(1000,1400,1900)$ for $R$. The linear scale of the colour coding may be used for interpolation 
Table 30. Large scale surface photometries of the Milky Way in the visual/near visual spectral domain (in addition to those displayed as Figs. 63- 67)

\begin{tabular}{ccrl}
\hline Spectral range & \multicolumn{2}{c}{$\begin{array}{l}\text { Approximate interval of galactic } \\
\text { longitudes }\end{array}$} & Reference \\
& $0 \ldots 360^{\circ}$ & $-90 \ldots+90^{\circ}$ & Elsässer \& Haug (1960) \\
\hline$P, V$ & $0 \ldots 360^{\circ}$ & $-20 \ldots+20^{\circ}$ & Smith et al. (1970) \\
$530 \pm 15 \mathrm{~nm}$ & $0 \ldots 360^{\circ *}$ & $-50 \ldots+50^{\circ *}$ & Pfleiderer \& Mayer (1971) \\
$U$ & $0 \ldots 360^{\circ *}$ & $-90 \ldots+90^{\circ *}$ & Classen (1976) \\
$B$ & \multicolumn{2}{c}{ Northern Milky Way } & Zavarzin $(1978)$ \\
$710 \pm 100 \mathrm{~nm}$ & $0 \ldots 360^{\circ}$ & $-90 \ldots-55^{\circ}$ & Weinberg $(1981)^{+}$ \\
$440 \pm 45 \mathrm{~nm}$ & $0 \ldots 360^{\circ}$ & $-90 \ldots-55^{\circ}$ & Weinberg $(1981)^{+}$ \\
$640 \pm 50 \mathrm{~nm}$ & $41 \ldots 210^{\circ}$ & $-41 \ldots+41^{\circ}$ & Winkler et al. $(1981)$ \\
$356 \pm 53 \mathrm{~nm}$ & $0 \ldots 360^{\circ}$ & $-15 \ldots+15^{\circ}$ & Toller $(1990)^{+}$ \\
$B-R=440 \mathrm{~nm}-640 \mathrm{~nm}$ & $0 \ldots 360^{\circ}$ & $-90 \ldots+90^{\circ}$ & Wicenec $(1995)^{+}$, Wicenec \& van Leeuwen $(1995)^{+}$ \\
\hline$B, V$ & &
\end{tabular}

*as far as visible from about $30^{\circ}$ southern geographical latitude,

${ }^{+}$space experiments, included here for comparison, see a detailed presentation of Pioneer 10 results in Sect. 10.4.

Note: The earlier photometries by Elsässer and Haug and by Smith et al. are included here only for comparison. It is recommended to refer to the space-based photometries, to the Bochum photometries shown in Figs. 63-67 and to the later photometries of this table.

Table 31. Surface photometries covering smaller areas of the Milky Way

\begin{tabular}{ccrl}
\hline Spectral range & \multicolumn{2}{c}{$\begin{array}{c}\text { Approximate interval of galactic } \\
\text { longitudes }\end{array}$} & Reference \\
latitudes & \\
\hline$B$ & $295 \ldots 310^{\circ}$ & $-6 \ldots+5^{\circ}$ & Mattila (1973) \\
$U$ & $-63 \ldots+30^{\circ}$ & $-30 \ldots+30^{\circ}$ & Pröll (1980) \\
$U, B, V$ & \multicolumn{2}{c}{ Scorpius } & Hanner et al. (1978) ${ }^{a}$ \\
$U, B, V$ & \multicolumn{2}{c}{ selected scans } & Leinert \& Richter $(1981)^{a}$ \\
$U, B, V, R$ & $289 \ldots 316^{\circ}$ & $-15 \ldots+14^{\circ}$ & Seidensticker et al. (1982) \\
\hline
\end{tabular}

${ }^{a}$ space experiment, included here for comparison, since well-calibrated.

most of the sky (see Fig. 68) except for a region near the spin axis of the spacecraft (within $30^{\circ}$ of the sun). Table 33 presents a log of observations with the Pioneer 10 IPP. A similar schedule was performed with the IPP on Pioneer 11, starting in April 1973. The combined data provide a higher spatial resolution than would have been possible to obtain with a single map or with observations from a single spacecraft (S/C). Further, Pioneer 11 obtained 12 additional maps between November 1981 and December 1982 to "fill in" the aforementioned sky gap regions.

The instantaneous field of view of each IPP was approximately $2.3^{\circ}$ square. Brightness was integrated for $1 / 64^{\text {th }}$ (one sector) of the $12.5 \mathrm{~s}$ spacecraft spin period, giving a maximum effective FOV of $2.3^{\circ} \times 7.9^{\circ}$ when the telescope was perpendicular to the spin axis $\left(\mathrm{LA}=90^{\circ}\right)$. The spin axis was directed more or less toward the sun. By moving the IPP telescope in steps of $1.8^{\circ}$ in look angle, the entire sky between $29^{\circ}$ and $170^{\circ}$ from the spin axis could be scanned. The spinning, sectoring and step- ping resulted in a two-dimensional overlapping pattern of FOV's on the sky for each map (see Fig. 68). Since the spin axis moved slowly on the celestial sphere according to the moving spacecraft position, most of the sky was eventually covered with a resolution better than the $1.8^{\circ}$ roll-to-roll separation of FOV's in a single map.

The data reduction methodology is described in a User's Guide (Weinberg \& Schuerman 1981) for the Pioneer 10 and Pioneer 11 IPP data archived at the National Space Science Data Center (NSSDC). Signals of bright stars were used to calibrate the decaying sensitivity of the IPP channels. Individually resolved stars, typically those brighter than $6.5 \mathrm{mag}$, were removed from the measured brightnesses on the basis of a custom made catalog containing 12457 stars. The absolute calibration was based on the instrument's response to Vega. Finally, the Pioneer 10 and 11 blue and red data were represented in $S 10_{\odot}$ units. The result is a background sky tape, which, for the data beyond 2.8 AU, contains the 

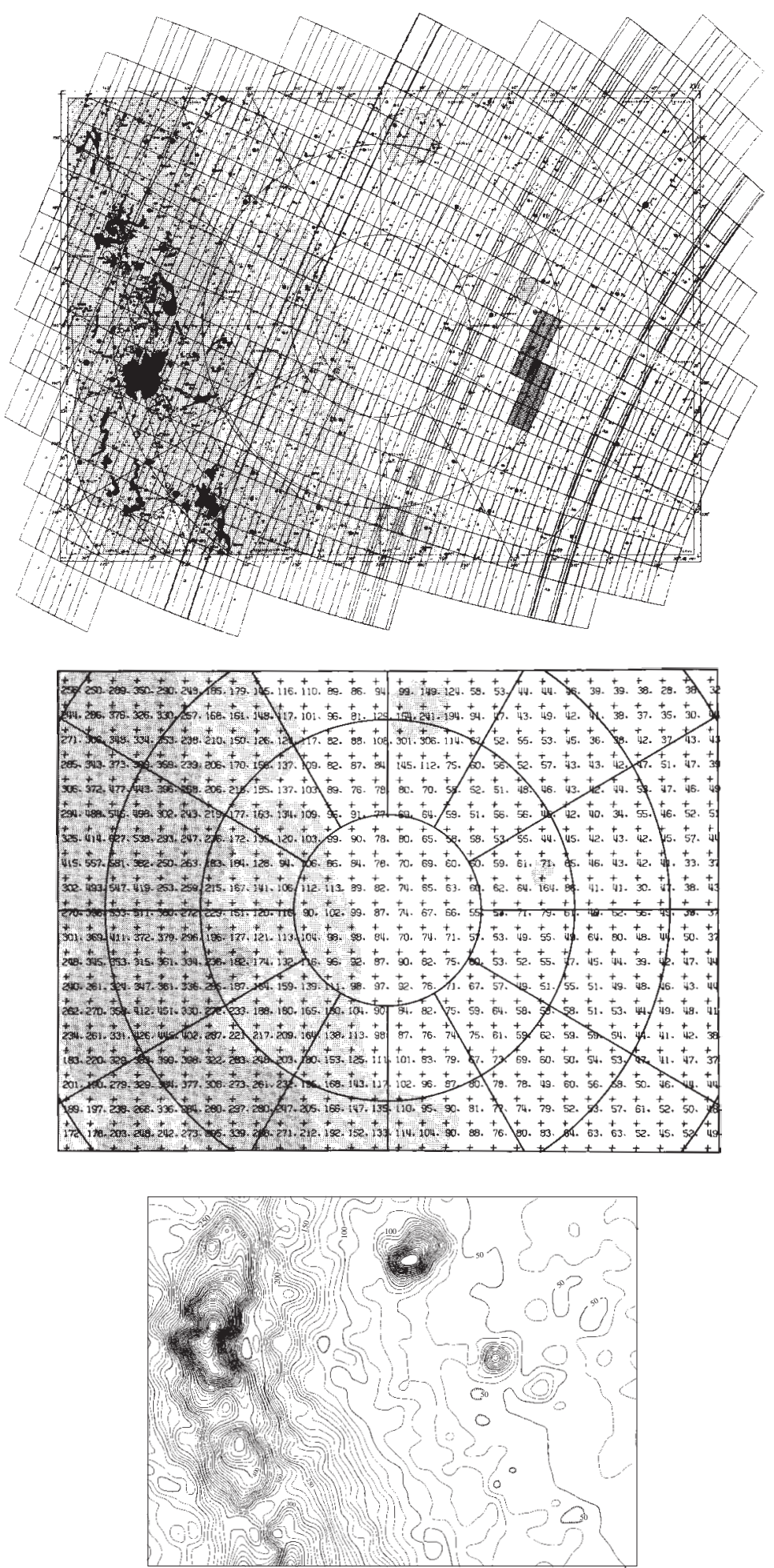

Fig. 68. Example for Pioneer data in the blue $(440 \mathrm{~nm})$, from a sky map observed beyond 3 AU. Upper panel: Map of the Becvar atlas showing part of the southern Milky Way and the Magellanic clouds, with the sectored field-of-view of Pioneer 10 overlaid. Middle panel: Brightness values in $S 1 \odot_{\odot}$ units interpolated from the individual sector brightnesses to a rectangular coordinate grid. Lower panel: Isocontour map constructed from this set of brightnesses 

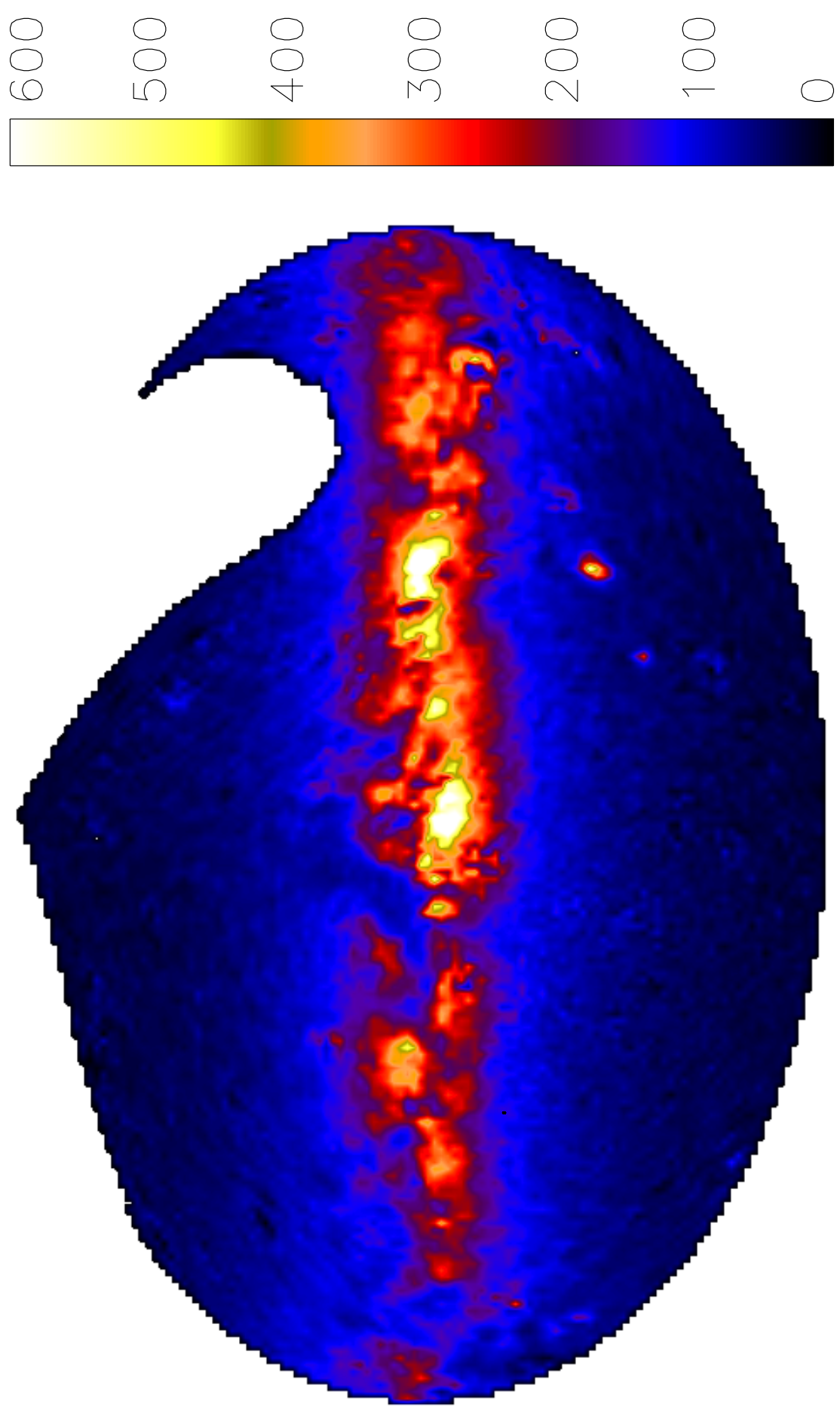

Fig. 69. Pioneer $10 / 11$ blue sky map at $440 \mathrm{~nm}$ at $0.5^{\circ}$ resolution, constructed from Pioneer 10 and Pioneer 11 maps taken at 3.26 AU to 5.15 AU heliocentric distance. The map is in Aitoff projection. The galactic center is at the center. From Gordon (1997) 
Table 32. Comparison of the Bochum $U B V R$ photometries (denoted as " $X$ ") with other photometries

\begin{tabular}{|c|c|}
\hline$U$ passband & $\left(\lambda_{\mathrm{bi}}=352 \mathrm{~nm}, W_{\lambda}=51 \mathrm{~nm}, \Delta \lambda=97 \mathrm{~nm}\right)$ \\
\hline & $=(0.97 \pm 0.18) \cdot X-(12 \pm 4)$ \\
\hline & $=(1.11 \pm 0.12) \cdot X$ \\
\hline & $=(0.84 \pm 0.02) \cdot X-(10 \pm 3)$ \\
\hline & $=(1.19 \pm 0.03) \cdot X-(28 \pm 5)$ \\
\hline$B$ passband & $\left(\lambda_{\mathrm{bi}}=421 \mathrm{~nm}, W_{\lambda}=80 \mathrm{~nm}, \Delta \lambda=141 \mathrm{~nm}\right)$ \\
\hline & $=(0.83 \pm 0.04) \cdot X+(23 \pm 5)$ \\
\hline & Leinert \& Richter (1981) \\
\hline & $=(0.82 \pm 0.12) \cdot X+(31 \pm 23)$ \\
\hline & $=(1.18 \pm 0.03) \cdot X+(16 \pm 3)$ \\
\hline & $=(0.90) \cdot X+25$ \\
\hline$V$ passband & $\left(\lambda_{\mathrm{bi}}=530 \mathrm{~nm}, W_{\lambda}=94 \mathrm{~nm}, \Delta \lambda=159 \mathrm{~nm}\right)$ \\
\hline & Dachs $(1970)$ \\
\hline & $=(0.64 \pm 0.13) \cdot X+(36 \pm 8)$ \\
\hline & Leinert \& Richter (1981) \\
\hline & $=(1.13 \pm 0.09) \cdot X-(88 \pm 14)$ \\
\hline$R$ passband & $\left(\lambda_{\mathrm{bi}}=678 \mathrm{~nm}, W_{\lambda}=24 \mathrm{~nm}, \Delta \lambda=53 \mathrm{~nm}\right)$ \\
\hline & Seidensticker et al. $(1982)=(1.09 \pm 0.04) \cdot X-(464 \pm 36)$ \\
\hline
\end{tabular}

$\lambda_{\mathrm{bi}}$ is the wavelength which bisects the recorded energy for this filter, $W_{\lambda}$ the equivalent width and $\Delta_{\lambda}$ the total width of the passband.

Please note: $1 \sigma$ mean errors given only if data permit (muliplicative term) and/or mean differs by more than $1 \sigma$ from zero (additive term).

The Bochum $U B V R$ photometries are stored at the Strasbourg Centre de Données Stellaires (CDS) under http://cdsweb.ustrasbg.fr/htbin/myqcat3?VII/199/

integrated starlight, including the contribution from the diffuse galactic light. A more complete description of the reduction and use of the data is being prepared (Weinberg, Toller, and Gordon). The data can be accessed under http://nssdc.gsfc.nasa.gov/, following from there on the topics "Master catalog", "Pioneer 10" and "Experiment information".

The background sky data set can be addressed in a variety of ways, including overlaying the data on a sky atlas such as Becvar's Atlas Coeli (1962), interpolating the posted data on an evenly spaced coordinate grid, and contouring the data. Each of these is shown in the three panels of Fig. 68, all covering the south celestial pole region, which includes low galactic latitude regions and both the Small and Large Magellanic Clouds. The map scale and magnitude limit of the Atlas Coeli make this atlas convenient for illustrating and manipulating Pioneer background sky data. The upper panel in Fig. 68 shows a single Pioneer 10 map's pattern of FOV's overlaid on the corresponding region of the Becvar atlas. The map shows the overlap in both look angle and sector (day 68 of year 1974, observed at $R=5.15 \mathrm{AU}$ ). The middle panel shows the result of interpolating the data for six map days of observations in blue on an evenly spaced coordinate grid for the same region of sky. We estimate that the random error in the numbers shown in the middle panel is 2 to 3 $S 10_{\odot}$ units, and perhaps 5 units in the Milky Way and the Magellanic clouds. An isophote representation of the data (lower panel) is perhaps the most convenient way to present the data. The interval between isophotes is 5 $S 10_{\odot}$ units. The spatial resolution was found to be approximately $2^{\circ}$. Regularly spaced grid values of Pioneer 10 blue and red brightnesses were determined in this manner for the entire sky, from which data were derived every two degrees both in galactic and equatorial coordinates. Part of these data are used in Tables 35 to 38 to depict Pioneer 10 blue and red data at $10^{\circ}$ intervals in both coordinate systems. Pioneer 11 data showed no significant differences to Pioneer 10 data, so only Pioneer 10 data are discussed and shown here.

More recently, Gordon (1997) further analyzed Pioneer 10 and 11 data from beyond the asteroid belt. He also found no significant differences between the Pioneer 10 and 11 data. His grey scale presentation of the combined data with $0.5^{\circ}$ spatial resolution is shown in an Aitoff projection in Fig. 69. The gap in this figure corresponds to that discussed earlier. Gordon did not have available those special data sets closing the gap. 
Table 33. Log of cruise phase observations with the Pioneer 10 imaging photopolarimeter

\begin{tabular}{|c|c|c|c|c|c|c|c|}
\hline \multirow{3}{*}{$\begin{array}{l}\text { Year } \\
1972\end{array}$} & \multirow{3}{*}{$\begin{array}{r}\text { Calendar } \\
\text { Date } \\
\text { Mar. 10 }\end{array}$} & \multirow{2}{*}{$\begin{array}{c}\text { Sun-S/C } \\
\text { Distance } \\
(\mathrm{AU})\end{array}$} & \multirow{2}{*}{$\begin{array}{c}\text { S/C Distance } \\
\text { from ecliptic }^{a} \\
(\mathrm{AU})\end{array}$} & \multicolumn{2}{|c|}{ Heliocentric } & \multirow{2}{*}{$\begin{array}{c}\text { Usable LA }^{b} \\
\text { Range } \\
\text { (deg) }\end{array}$} & \multirow{2}{*}{$\begin{array}{c}\text { Signal/ } \\
\text { Noise }\end{array}$} \\
\hline & & & & \multicolumn{2}{|c|}{$\beta_{\mathrm{S} / \mathrm{C}} \lambda_{\mathrm{deg})} \lambda_{\mathrm{S} / \mathrm{C}}$} & & \\
\hline & & 1.002 & -.0065 & -0.37 & 172.85 & $152-168$ & 8.70 \\
\hline & 11 & 1.004 & -.0073 & -0.41 & 174.12 & $135-167$ & 4.15 \\
\hline & 12 & 1.006 & -.00805 & -0.46 & 175.36 & $136-169$ & 7.68 \\
\hline & 14 & 1.011 & -.0095 & -0.54 & 177.63 & $128-169$ & 7.00 \\
\hline & 15 & 1.014 & -.0103 & -0.58 & 178.87 & $128-168$ & 5.65 \\
\hline & 16 & 1.017 & -.01107 & -0.62 & 180.07 & $128-169$ & 4.93 \\
\hline & 20 & 1.032 & -.01428 & -0.79 & 185.03 & $128-150$ & 5.62 \\
\hline & 22 & 1.040 & -.01581 & -0.87 & 187.37 & $128-168$ & 5.00 \\
\hline & 23 & 1.046 & -.01677 & -0.92 & 188.84 & $128-170$ & 4.56 \\
\hline & 29 & 1.075 & -.02117 & -1.13 & 195.47 & $128-169$ & 6.43 \\
\hline & 31 & 1.087 & -.0227 & -1.20 & 197.78 & $110-146$ & 5.87 \\
\hline & Apr. 4 & 1.110 & -.02554 & -1.32 & 201.95 & $110-170$ & 4.93 \\
\hline & 10 & 1.150 & -.02971 & -1.48 & 207.98 & $91-159$ & 4.12 \\
\hline & 13 & 1.171 & -.03165 & -1.55 & 210.76 & $91-170$ & 3.78 \\
\hline & 17 & 1.201 & -.03424 & -1.63 & 214.39 & $91-166$ & 3.15 \\
\hline & 20 & 1.224 & -.03613 & -1.69 & 217.02 & $91-168$ & 4.71 \\
\hline & 27 & 1.281 & -.04027 & -1.80 & 222.68 & $86-103$ & 4.09 \\
\hline & 28 & 1.289 & -.04086 & -1.82 & 223.48 & $91-158$ & 3.75 \\
\hline & May 5 & 1.349 & -.04474 & -1.90 & 228.64 & $46-168$ & 11.46 \\
\hline & 8 & 1.376 & -.04632 & -1.93 & 230.73 & $46-156$ & 14.43 \\
\hline & 17 & 1.453 & -.05058 & -1.99 & 236.23 & $46-170$ & 13.87 \\
\hline & 30 & 1.586 & -.05694 & -2.06 & 244.26 & $46-169$ & 10.34 \\
\hline & June 7 & 1.652 & -.05972 & -2.07 & 247.70 & $46-169$ & 8.90 \\
\hline & 13 & 1.709 & -.06196 & -2.08 & 250.46 & $49-130$ & 7.75 \\
\hline & 20 & 1.774 & -.06436 & -2.08 & 253.40 & $42-067$ & 7.96 \\
\hline & 22 & 1.788 & -.06485 & -2.08 & 254.00 & $44-170$ & 7.31 \\
\hline & 27 & 1.841 & -.06666 & -2.08 & 256.21 & $\begin{array}{c}91-130 \\
141-168\end{array}$ & 4.21 \\
\hline & 29 & 1.861 & -.0673 & -2.07 & 257.00 & $68-169$ & 5.21 \\
\hline & July 21 & 2.062 & -.07326 & -2.04 & 264.32 & 128 & 3.87 \\
\hline & 24 & 2.090 & -.07399 & -2.03 & 265.22 & 128 & 6.40 \\
\hline & 27 & 2.117 & -.07469 & -2.02 & 266.11 & 128 & 4.34 \\
\hline & 31 & 2.152 & -.07557 & -2.01 & 267.21 & 128 & 7.75 \\
\hline & Aug. 3 & 2.179 & -.07622 & -2.00 & 268.04 & $\begin{array}{l}104-137 \\
160-170\end{array}$ & 4.81 \\
\hline & 10 & 2.241 & -.07766 & -1.99 & 269.87 & 128 & 5.00 \\
\hline & 11 & 2.249 & -.07784 & -1.98 & 270.11 & $\begin{array}{c}91-140 \\
158-170\end{array}$ & 4.43 \\
\hline & 16 & 2.294 & -.07880 & -1.97 & 271.36 & $91-168$ & 6.31 \\
\hline & 23 & 2.354 & -.08005 & -1.95 & 273.01 & $\begin{array}{c}74-145 \\
167-170\end{array}$ & 5.90 \\
\hline & 30 & 2.413 & -.08121 & -1.93 & 274.58 & $76-158$ & 4.03 \\
\hline & Sept. 5 & 2.467 & -.08219 & -1.91 & 275.94 & $76-166$ & 5.09 \\
\hline & 8 & 2.492 & -.08263 & -1.90 & 276.56 & $128-169$ & 4.59 \\
\hline & 26 & 2.640 & -.085005 & -1.85 & 280.07 & $76-105$ & \\
\hline & 27 & 2.641 & -.08502 & -1.84 & 280.09 & $76-150$ & 7.34 \\
\hline & Oct. 10 & 2.750 & -.08652 & -1.80 & 282.50 & $49-79$ & 5.15 \\
\hline & Oct. 18 & 2.812 & -.08728 & -1.78 & 283.81 & $\begin{array}{l}(42-68)^{*} \\
(77-163)^{*}\end{array}$ & 4.78 \\
\hline & 19 & 2.821 & -.08739 & -1.78 & 283.99 & $(91-157)^{*}$ & 4.71 \\
\hline & Nov. 4 & 2.939 & -.08865 & -1.73 & 286.37 & $42-161$ & 4.93 \\
\hline & 19 & 3.056 & -.08969 & -1.68 & 288.63 & $43-173$ & 2.62 \\
\hline & Dec. 4 & 3.163 & -.09046 & -1.64 & 290.61 & $42-170$ & 2.90 \\
\hline & 19 & 3.269 & -.09104 & -1.60 & 292.47 & $40-170$ & 5.15 \\
\hline
\end{tabular}


Table 33. continued

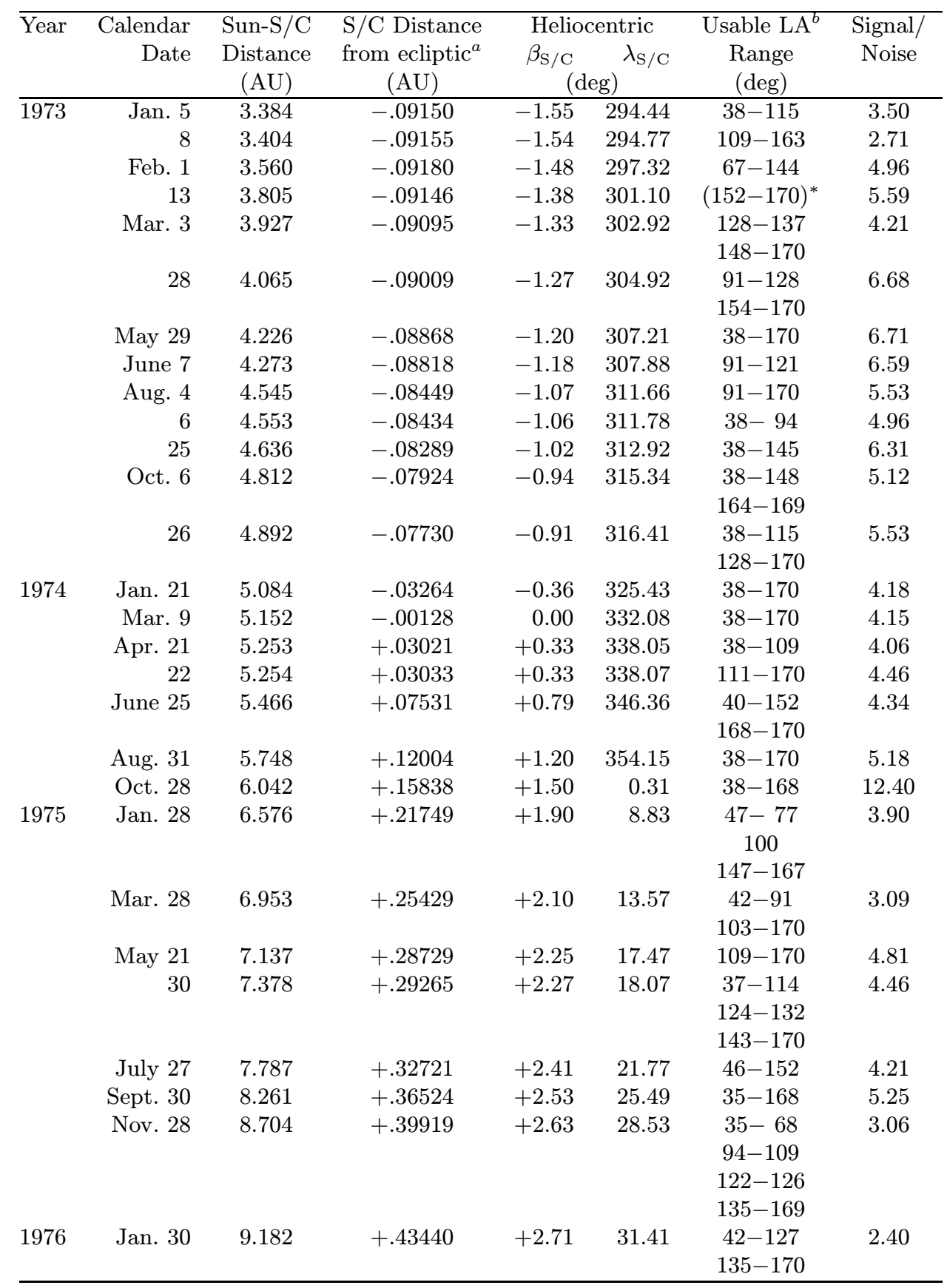

${ }^{a}$ positive values mean a location of the spacecraft north of the ecliptic plane.

${ }^{b} \mathrm{LA}=$ look angle $=$ angle measured from the spin axis.

${ }^{*}$ Approximately $1 / 2$ of the data are lost due to low data rate and other factors.

From the Pioneer data, blue and red brightnesses at the celestial, ecliptic and galactic poles were derived from isophote maps of the polar regions like the one shown for the south celestial pole in Fig. 68. They are compared with other photometric data and with star counts in Table 34. There is fair agreement among the photometries. However, because of the lack of atmospheric and interplanetary sig- nals in the Pioneer data, these data should be preferred over the other photometries when determining the level of galactic light in a certain region. Generally the photometries are at higher levels than the star counts, as one would expect, since the photometries contain the contributions of diffuse galactic light (Sect. 11) and extragalactic background light (Sect. 12). Equal numbers for the bright- 
Table 34. Brightnesses of background starlight and integrated starlight at the north and south celestial, ecliptic, and galactic poles (in $S 10_{\odot}$ units). Stars with $m_{V}<6.5$ excluded. Adapted from Toller et al. (1987)

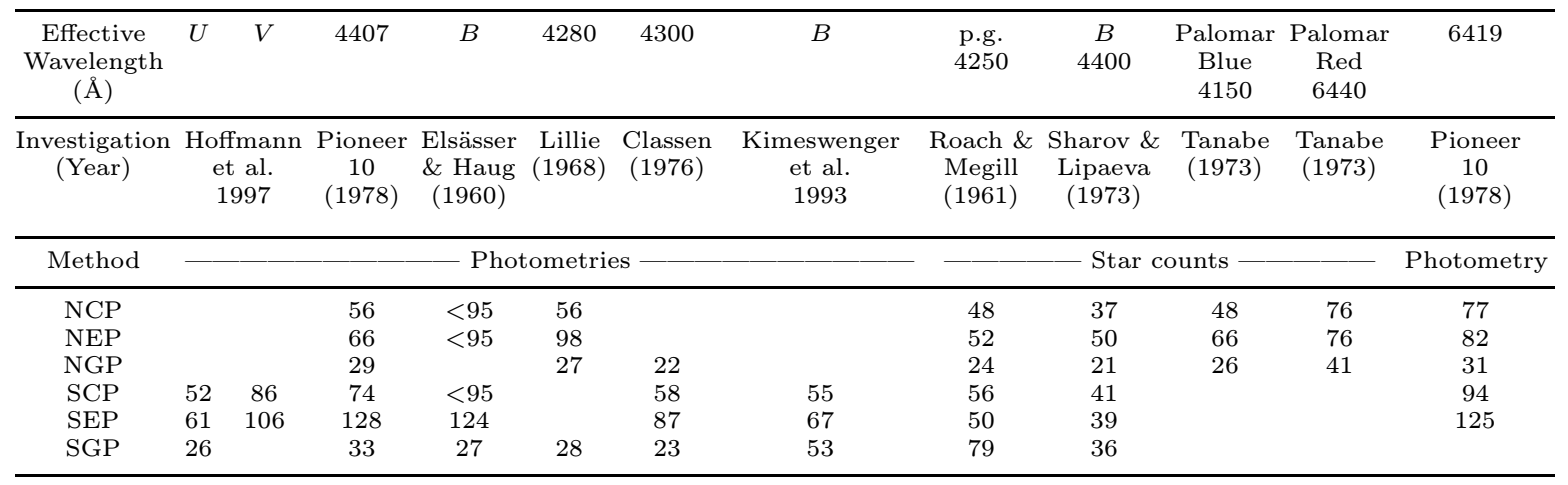

nesses in the blue and the red shown in Table 34 would mean that the galactic component of the night sky brightness has solar colour. The Pioneer data show a reddening at the poles, and this reddening appears all over the sky (see Tables 35-38). Details can be found in Toller (1981) and Toller (1990).

Pioneer blue data are compared in Fig. 70 to star counts and some earthbased photometric observations along four cuts through the Milky Way at different galactic longitudes. Generally, Classen's (1976) results agree quite well with the Pioneer data, also at high galactic latitudes, except at $l=30^{\circ}$ and at middle latitudes where they are much lower, possibly from errors in the difficult corrections for airglow and atmospheric extinction, since her observations of this region had to be made near the horizon (from South Africa). Generally speaking, all ground-based data sets suffer from the presence of airglow continuum and some from line emission (see Sect. 6) in the instrument's spectral band (see Weinberg \& Mann 1967). However, ground-based photometries may offer better spatial resolution, like the Bochum Milky Way photometries presented in Sect. 10.3 above, and these particular photometries also give realistic absolute brightness values, as judged from their good agreement with the Helios $U, B$, $V$ photometry.

\subsection{Near- and mid-infrared}

Maps of the starlight distribution in the infrared are difficult to obtain. There are currently no sensitive, all-sky surveys of stars in the infrared, though the ground-based 2MASS and DENIS programs will provide that in the next several years. Extracting starlight maps from diffuse sky brightness measurements is challenging because of the need to separate the various contributions to the measured light. The COBE/DIRBE team has developed a detailed zodiacal light model which allows such a separation, at least in the near-infrared.
An all-sky image dominated by the stellar light of the Galaxy is presented in Fig. 71. The map was prepared by averaging 10 months of DIRBE data at $2.2 \mu \mathrm{m}$ wavelength after removal of the time-dependent signal from solar-system dust via a zodiacal light model. The remaining sky brightness at this wavelength is dominated by the cumulative light from $\mathrm{K}$ and $\mathrm{M}$ giants (Arendt et al. 1994), though individual bright sources can be detected at a level of about $15 \mathrm{Jy}$ above the local background in unconfused regions. Although this map also contains small contributions from starlight scattered by interstellar dust (cirrus) and any extragalactic emission, these contributions are much smaller than that from stars. No extinction correction has been applied to the map in Fig. 71; Arendt et al. (1994) found $2.2 \mu \mathrm{m}$ optical depths greater than 1 within $\approx 3^{\circ}$ of the Galactic plane for directions toward the inner Galaxy and bulge $\left(|l|<70^{\circ}\right)$. Arendt et al. used the multi-wavelength DIRBE maps to construct an extinction-corrected map over the central part of the Milky Way.

The typical appearance of the galactic stellar emission in the infrared the Milky Way is apparent in Fig. 71: because the interstellar extinction is much reduced in the infrared, this internal view of our Galaxy looks like a galaxy seen edge-on from the outside. Bulge and disk are clearly visible and separated. This appearance shows at all nearinfrared wavelengths (see Fig. 72).

To look at the starlight distribution over a broader spectral range, it is useful to concentrate on the low Galactic-latitude region. Figure 72 presents DIRBE maps of this region at $1.25 \mu \mathrm{m}, 2.2 \mu \mathrm{m}, 3.5 \mu \mathrm{m}$ and $4.9 \mu \mathrm{m}$, each with the zodiacal light removed using the DIRBE zodiacal light model. Since starlight is the dominant source at low latitudes over this spectral range, these maps are a good approximation to the infrared stellar light, with extinction of course decreasing as wavelength increases. Corresponding maps at 12 microns and longer are not shown, because at these wavelengths 
Table 35. Pioneer 10 background starlight in blue $(440 \mathrm{~nm})$, given in galactic coordinates and $S 10 \odot$ units. From Toller $(1981)$

\begin{tabular}{|c|c|}
\hline 8 & 成 \\
\hline 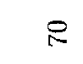 & 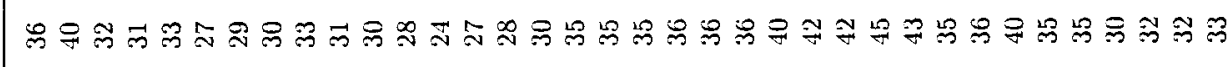 \\
\hline 8 & 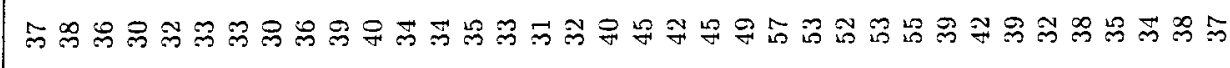 \\
\hline 品 & 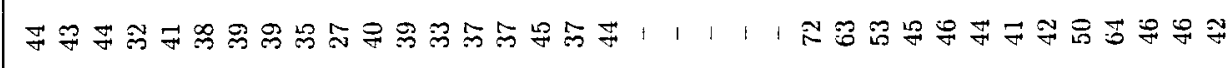 \\
\hline \& & 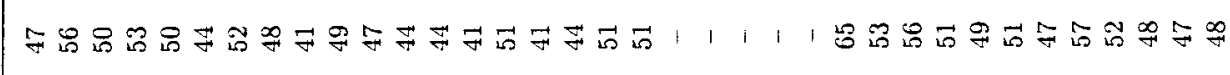 \\
\hline 尺 & 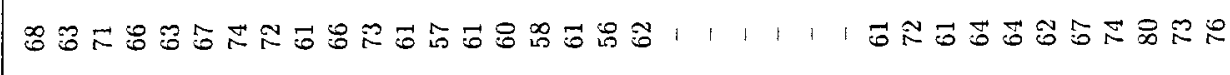 \\
\hline$\vec{\sim}$ & 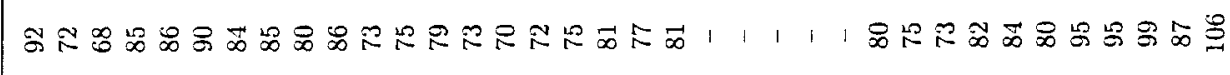 \\
\hline ฉ & 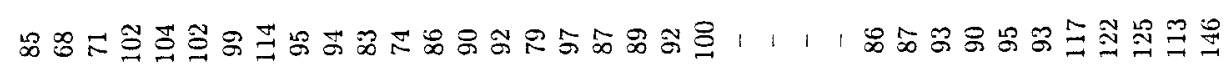 \\
\hline$\stackrel{0}{2}$ & 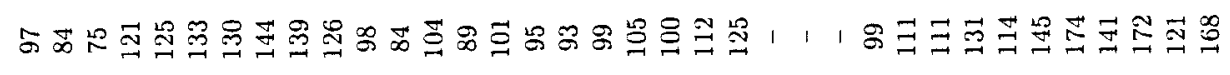 \\
\hline$\cong$ & 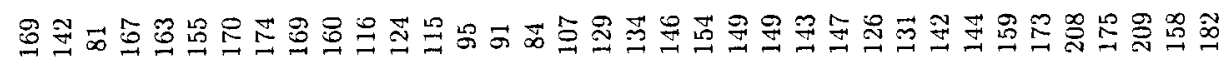 \\
\hline 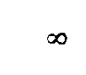 & 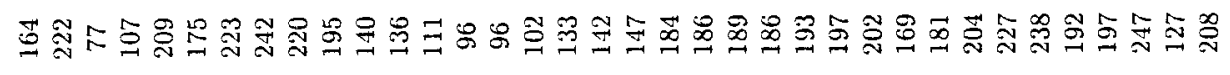 \\
\hline+ & 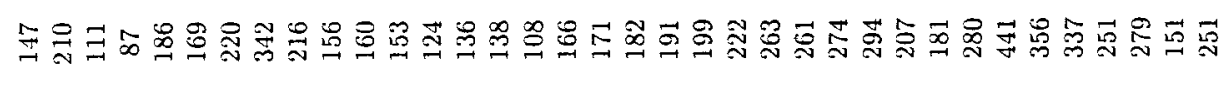 \\
\hline & 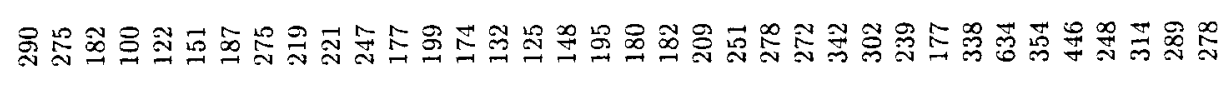 \\
\hline ir & 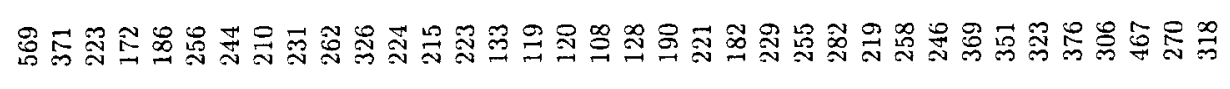 \\
\hline$\infty$ & 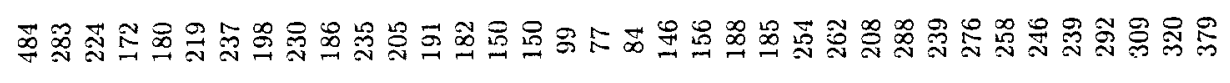 \\
\hline 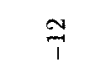 & 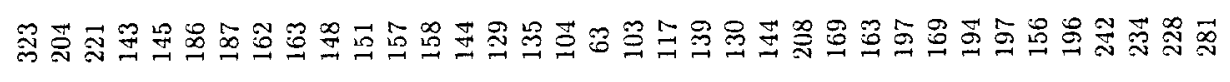 \\
\hline$\stackrel{0}{1}$ & 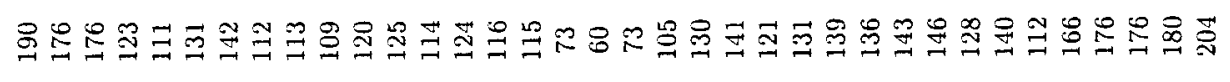 \\
\hline$\stackrel{8}{i}$ & 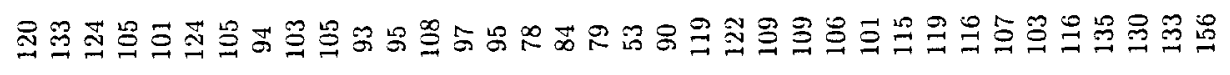 \\
\hline$\overleftrightarrow{\leftrightarrow}$ & 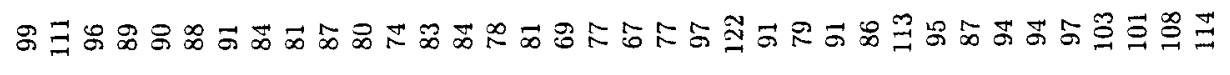 \\
\hline 空 & 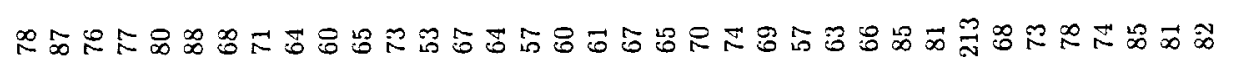 \\
\hline P & 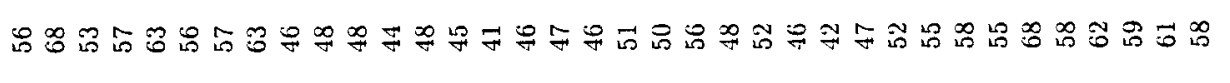 \\
\hline 䧄 & 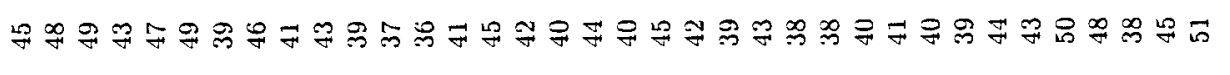 \\
\hline$\stackrel{0}{1}$ & 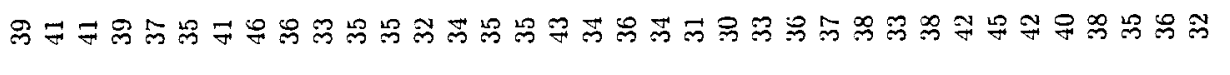 \\
\hline ? & 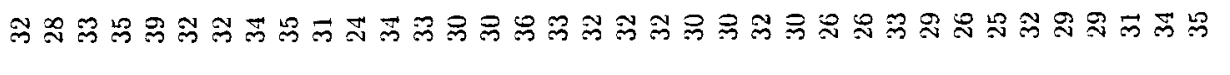 \\
\hline \$ి & 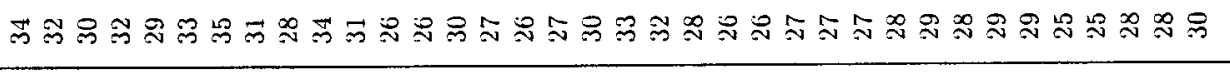 \\
\hline & N \\
\hline
\end{tabular}


Table 36. Pioneer 10 background starlight in red $(640 \mathrm{~nm})$, given in galactic coordinates and $S 10 \odot$ units. From Toller $(1981)$

\begin{tabular}{|c|c|}
\hline \& & 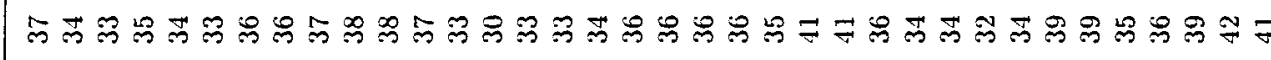 \\
\hline R & 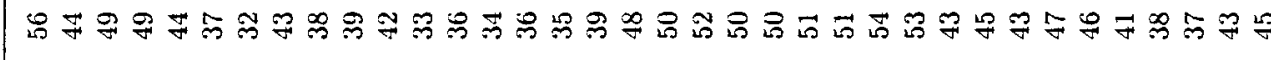 \\
\hline 0 & 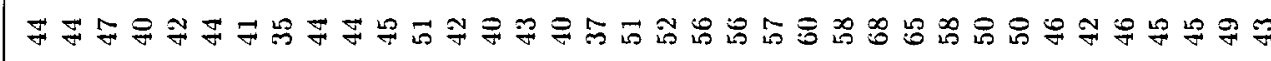 \\
\hline s. & 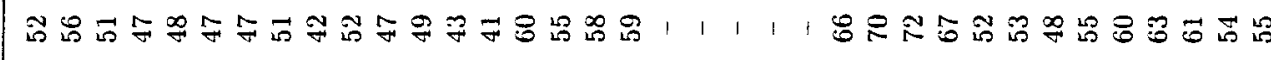 \\
\hline$\S$ & 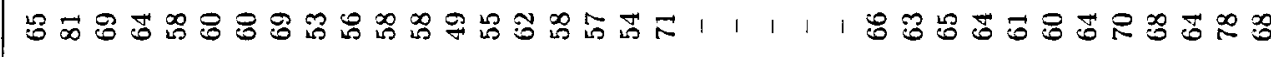 \\
\hline 征 & 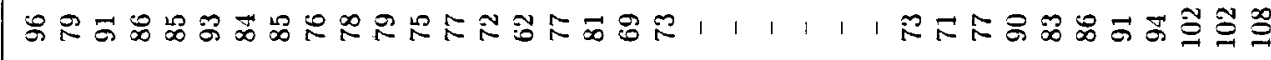 \\
\hline$\Xi$ & 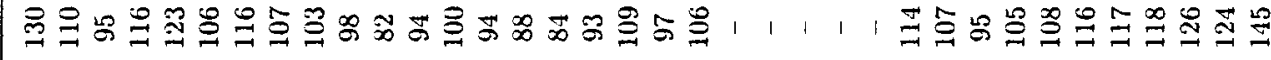 \\
\hline 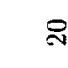 & 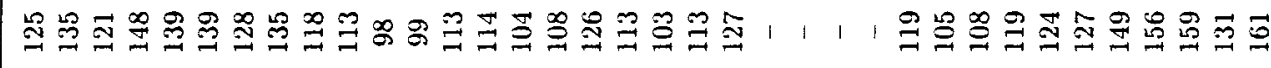 \\
\hline 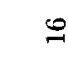 & 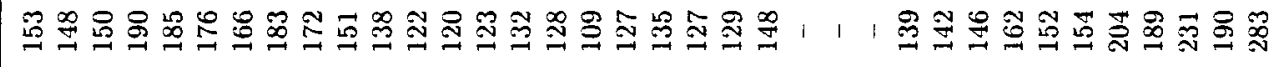 \\
\hline$\cong$ & 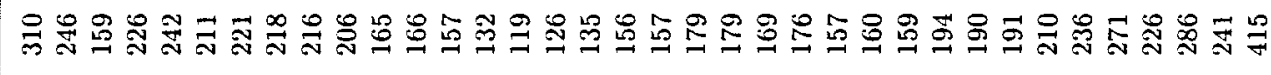 \\
\hline$\infty$ & 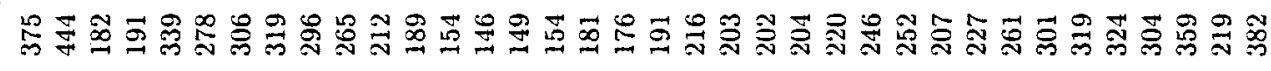 \\
\hline 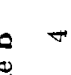 & 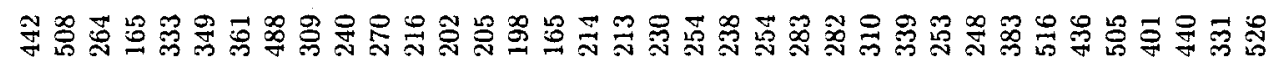 \\
\hline 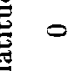 & 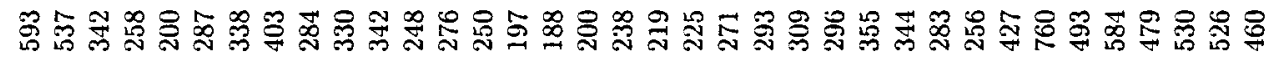 \\
\hline 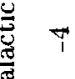 & 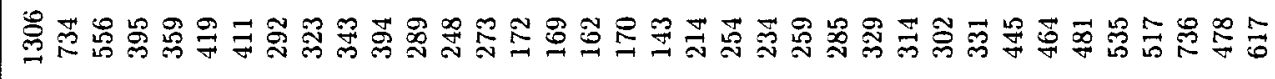 \\
\hline$\infty$ & 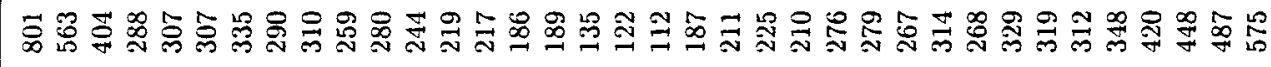 \\
\hline 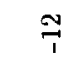 & 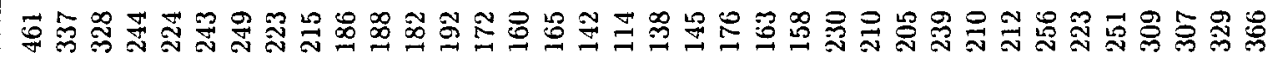 \\
\hline$\stackrel{0}{1}$ & 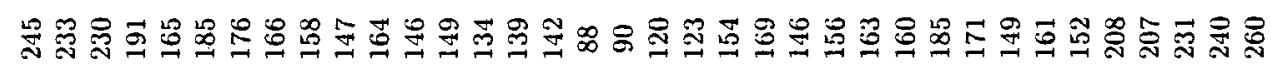 \\
\hline จి & 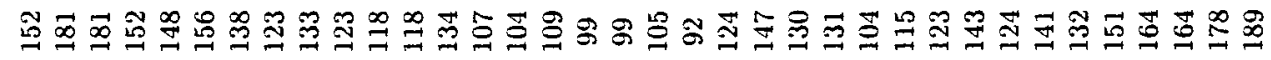 \\
\hline$\overparen{i}$ & 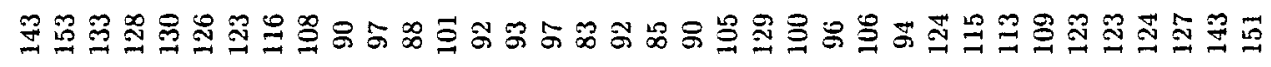 \\
\hline क् & 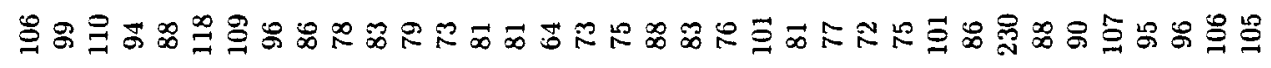 \\
\hline Pi & 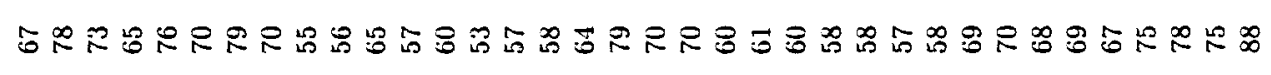 \\
\hline 草 & 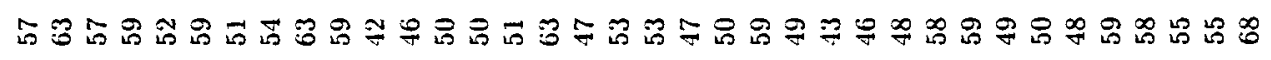 \\
\hline$\overbrace{i}$ & 施 충 员 \\
\hline R & 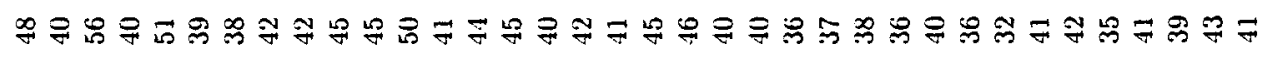 \\
\hline$\stackrel{\infty}{1}$ & 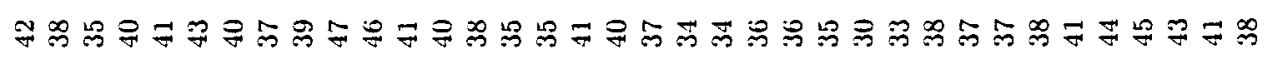 \\
\hline & \\
\hline
\end{tabular}


Table 37. Pioneer 10 background starlight in blue $(440 \mathrm{~nm})$, in equatorial coordinates and $S 10 \odot$ units. From Toller (1981)

\begin{tabular}{|c|c|}
\hline 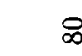 & 央冬 \\
\hline : & 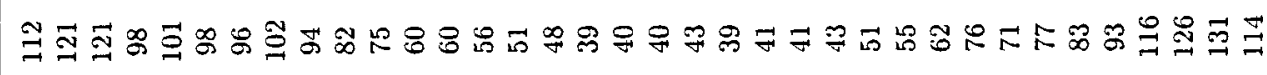 \\
\hline 8 & 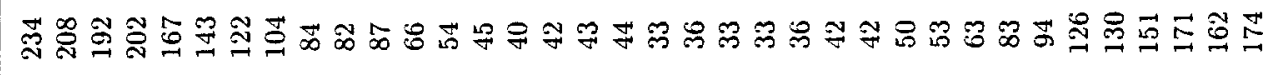 \\
\hline in & 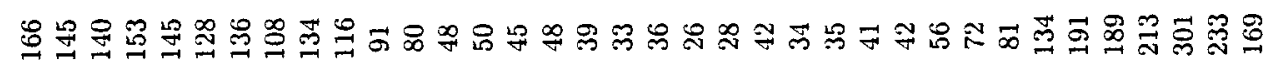 \\
\hline F & 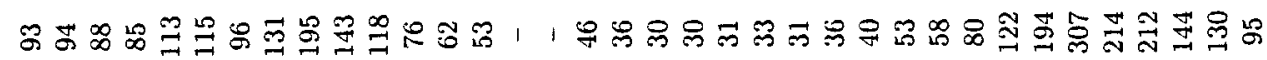 \\
\hline శ్ & 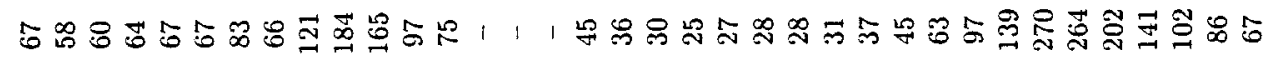 \\
\hline$\Xi$ & 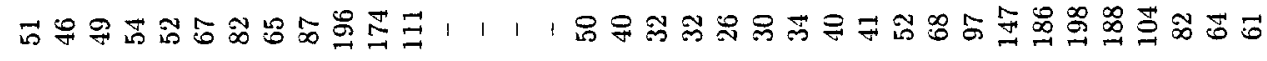 \\
\hline \& & 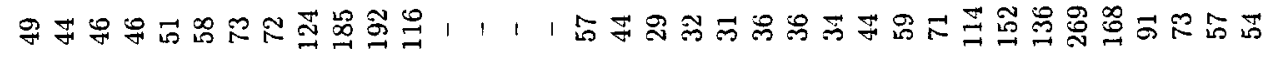 \\
\hline 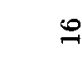 & 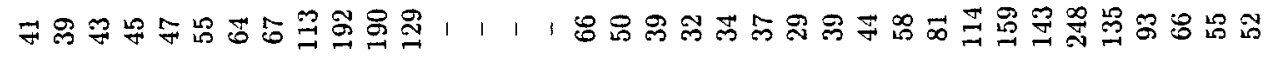 \\
\hline$\simeq$ & 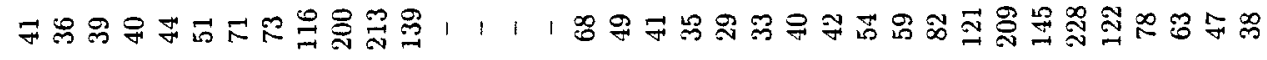 \\
\hline$\infty$ & 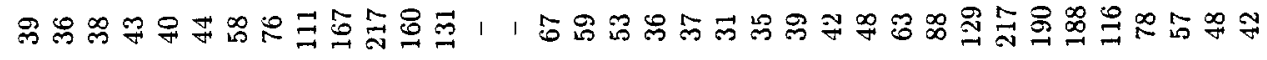 \\
\hline$\checkmark$ & 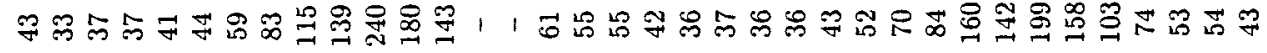 \\
\hline$=$ & 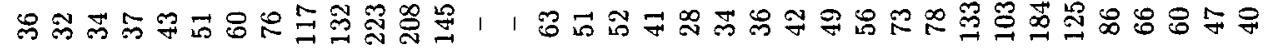 \\
\hline Ti & 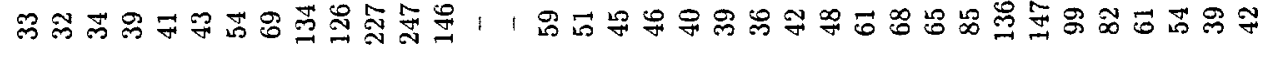 \\
\hline i & 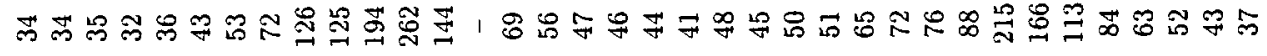 \\
\hline 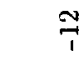 & 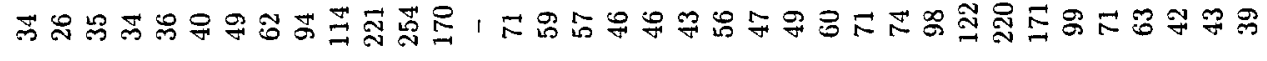 \\
\hline$\stackrel{9}{1}$ & 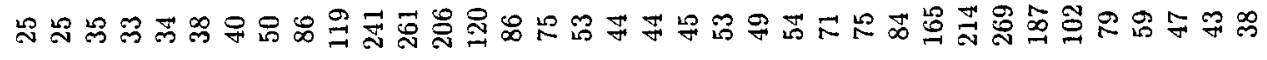 \\
\hline శ్ & 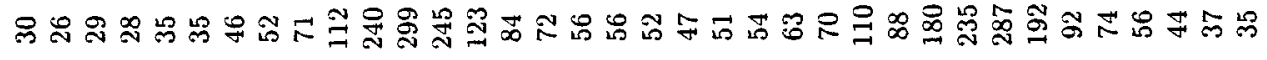 \\
\hline গু & 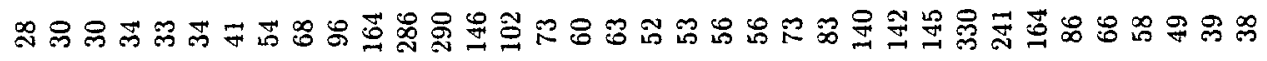 \\
\hline 宁 & 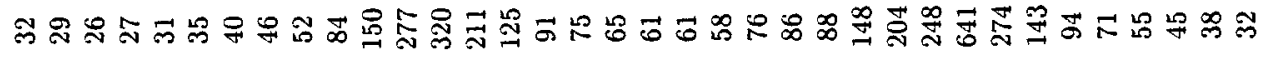 \\
\hline F & 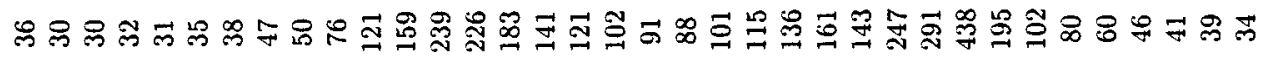 \\
\hline 号 & 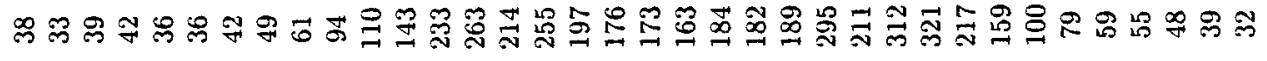 \\
\hline$\stackrel{8}{1}$ & 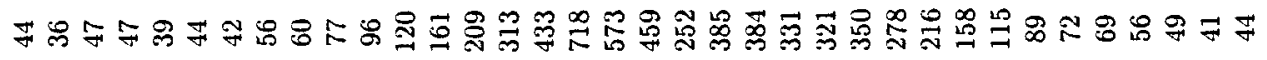 \\
\hline & 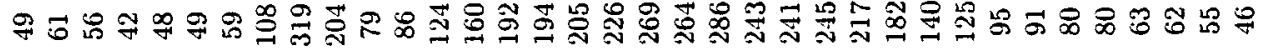 \\
\hline$\stackrel{\infty}{1}$ & 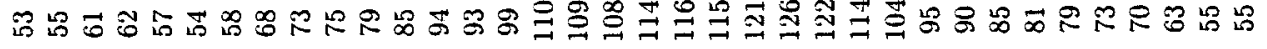 \\
\hline & \\
\hline
\end{tabular}


Table 38. Pioneer 10 background starlight in red $(640 \mathrm{~nm})$, in equatorial coordinates and $S 10_{\odot}$ units. From Toller $(1981)$

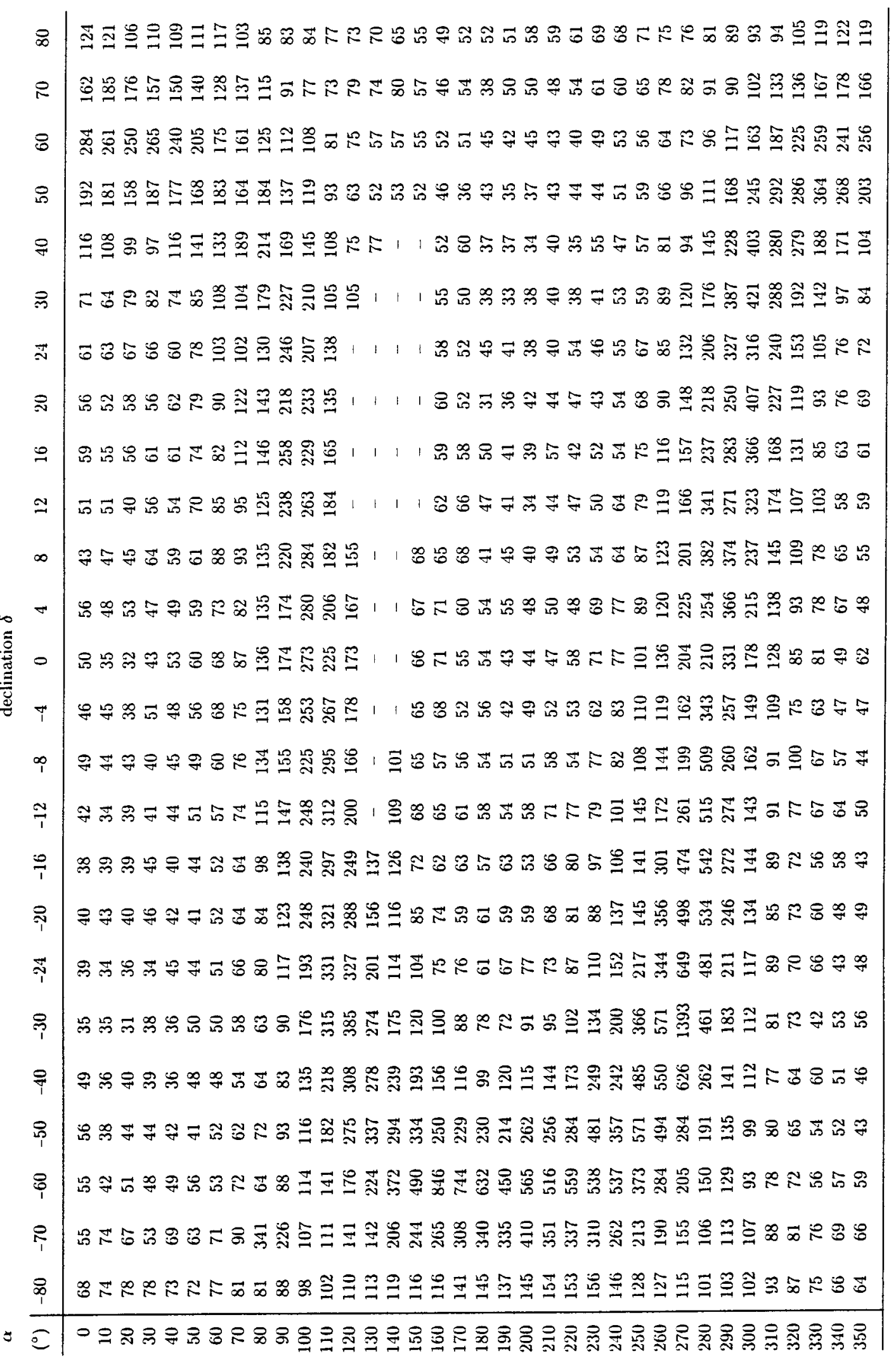




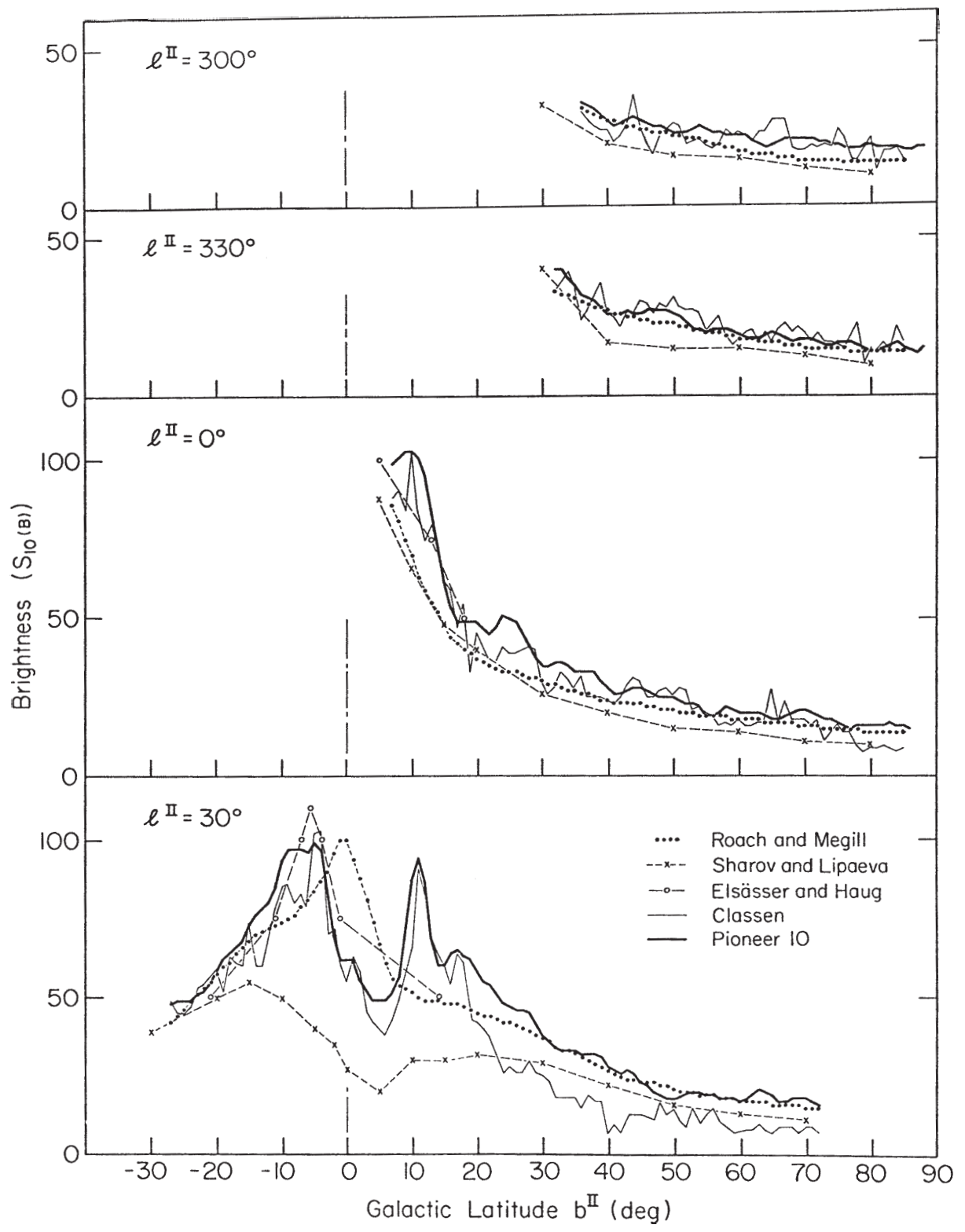

Fig. 70. Comparison of Pioneer 10 data for cuts through the Milky Way at different longitudes with the results of other investigations. Note that the ordinate is in $S_{10}(B)$ units. The numbers given in Table 35, which are given in $S 10 \odot$ units, are therefore larger by a factor corresponding to the solar $B-V$ value (compare Table 2)

interplanetary dust emission becomes the dominant contributor to sky brightness, and artifacts from imperfect removal of the zodiacal emission become more serious, as does the contribution from cirrus cloud emission. More elaborate modeling would be required to extract the stellar component of the sky brightness at these wavelengths. Figure 73 shows two sets of repesentative intensity profiles taken from the $1.25 \mu \mathrm{m}-4.9 \mu \mathrm{m}$ approximate "starlight" maps: the first set on a constant-latitude line near the Galactic plane and the second along the zerolongitude meridian. Full-sky DIRBE maps at $1.25 \mu \mathrm{m}$, $2.2 \mu \mathrm{m}, 3.5 \mu \mathrm{m}, 4.9 \mu \mathrm{m}, 12 \mu \mathrm{m}, 25 \mu \mathrm{m}, 60 \mu \mathrm{m}, 100 \mu \mathrm{m}$,
$140 \mu \mathrm{m}$, and $240 \mu \mathrm{m}$ with the zodiacal light removed, from which these approximate starlight maps have been selected, are available as the "Zodi-Subtracted Mission Average (ZSMA)" COBE data product, available from the NSSDC through the COBE homepage website at http://www.gsfc.nasa.gov/astro/cobe/cobe_home.html.

Model predictions for the integrated starlight, based on the galaxy model of Bahcall \& Soneira (1980), were given for the near- and mid-infrared as function of the brightness of the individually excluded stars by Franceschini et al. (1991b). Figures 74 and 75 show these results for wavelengths of $1.2 \mu \mathrm{m}, 2.2 \mu \mathrm{m}, 3.6 \mu \mathrm{m}$, and $12 \mu \mathrm{m}$. 


\section{$2.2 \mu \mathrm{m}$}

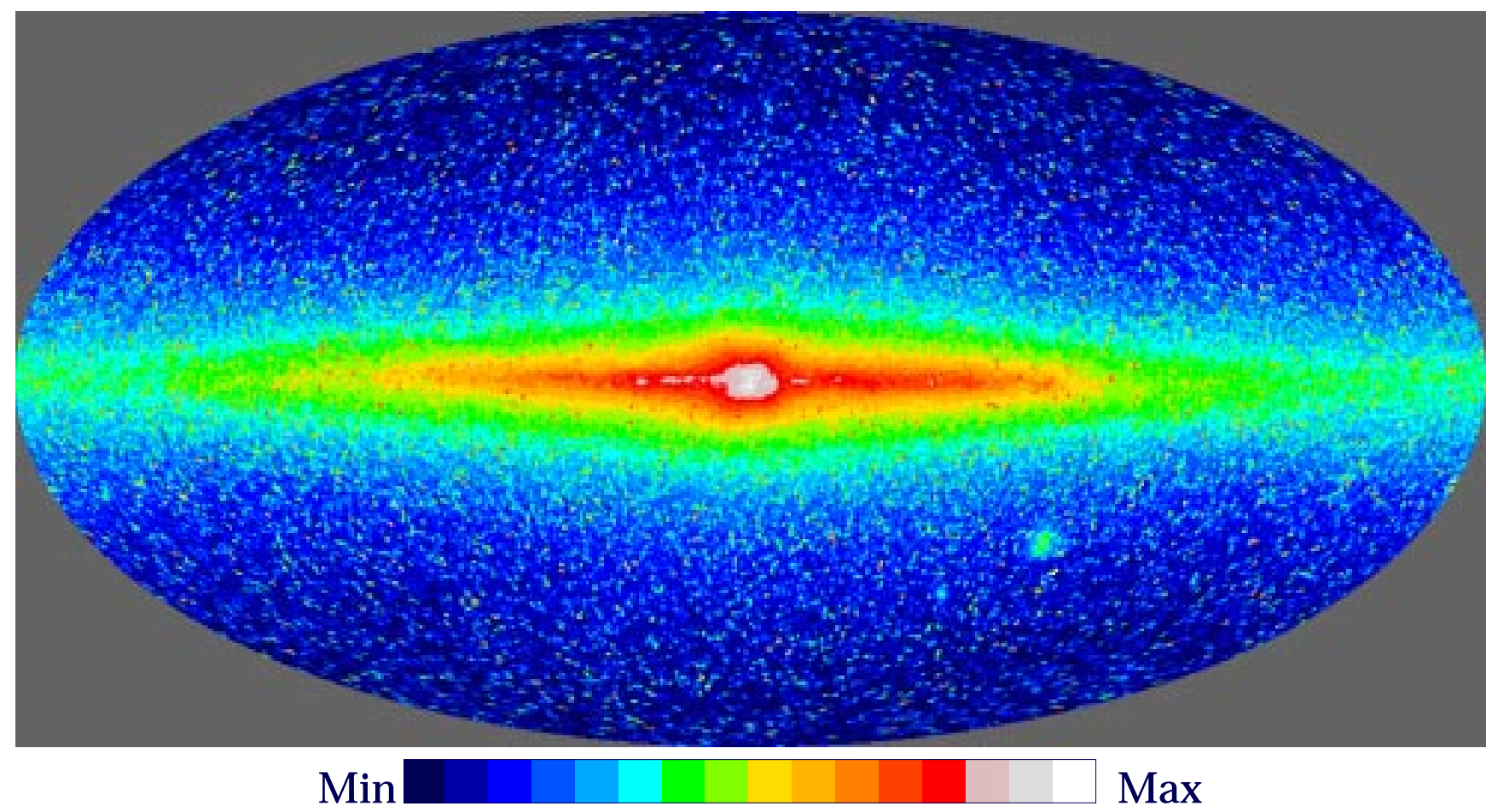

Fig. 71. DIRBE map of sky brightness at 2.2 microns in galactic coordinates, with zodiacal light removed. North is up, the galactic center in the middle, and galactic longitude increasing from right to left. This map is dominated by galactic starlight. No extinction correction has been made. Intensities are provided at 16 levels on a logarithmic scale ranging from 0.04 to $32 \mathrm{MJy} / \mathrm{sr}$. In detail these levels are: $0.040,0.062,0.097,0.15,0.24,0.37,0.57,0.90,1.40,2.19,3.41,5.33,8.32,12.98,20.26$, and 31.62 $\mathrm{MJy} / \mathrm{sr}$

\section{Diffuse galactic light}

\subsection{Overview}

Historically, the term Diffuse Galactic Light (DGL) denotes the diffuse component of the galactic background radiation which is produced by scattering of stellar photons by dust grains in interstellar space (Elvey \& Roach 1937; Roach \& Gordon 1973). This scattering process is the dominant contributor to the general interstellar extinction of starlight; thus, the DGL is most intense in directions where the dust column density and the integrated stellar emissivity are both high. This is generally the case at the lowest galactic latitudes, in all spectral regions extending from the far-ultraviolet into the nearinfrared. Typically, the DGL contributes between $20 \%$ to $30 \%$ of the total integrated light from the Milky Way.

However, for the purpose of this reference we are also interested in other sources of diffuse galactic background radiation, and they will be mentioned in the following where appropriate.

\subsection{Visual}

No comprehensive map of the DGL for the entire sky or even a significant fraction of the sky exists at this time. Groundbased observations in the visual face the difficult requirement that airglow, zodiacal light, and integrated starlight all need to be known to very high precision $\left( \pm 1 S_{10}(V)\right)$ if the DGL is to be derived by subtraction of the above components from the total sky brightness. In addition, the problems of atmospheric extinction and atmospheric scattering (Staude 1975) need to be solved.

Observations of the DGL at visual wavelengths carried out with rocket- or satellite-borne photometers still have the same major sources of uncertainty, i.e. the integrated starlight and the zodiacal light, remain principal contributors to the measured intensity.

The best prospect for a comprehensive measurement of the DGL in the visual was offered by the Pioneer 10 probe (see the more detailed description in Sect. 10.4), which carried out an all-sky photometric mapping in two wavebands centered near $440 \mathrm{~nm}$ and $640 \mathrm{~nm}$ from beyond the asteroid belt $(R>3 \mathrm{AU})$, where the contributions 


\section{$1.25 \mu \mathrm{m}$}

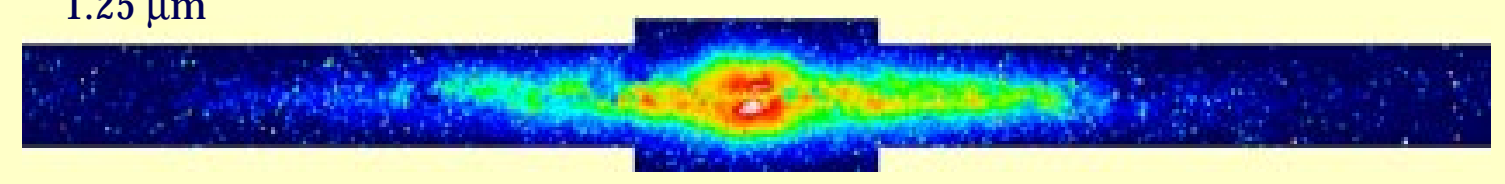

\section{$2.2 \mu \mathrm{m}$}
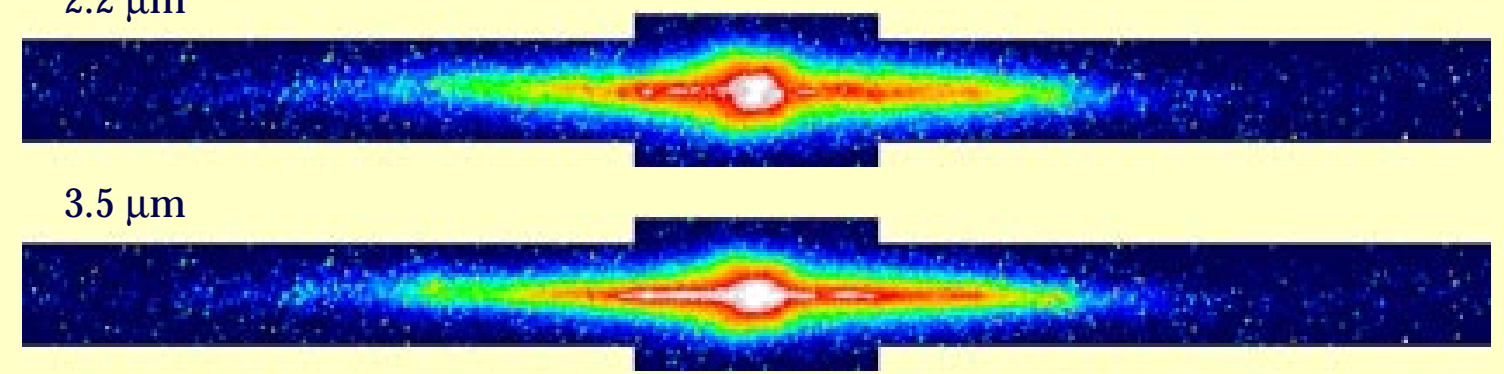

\section{$4.9 \mu \mathrm{m}$}

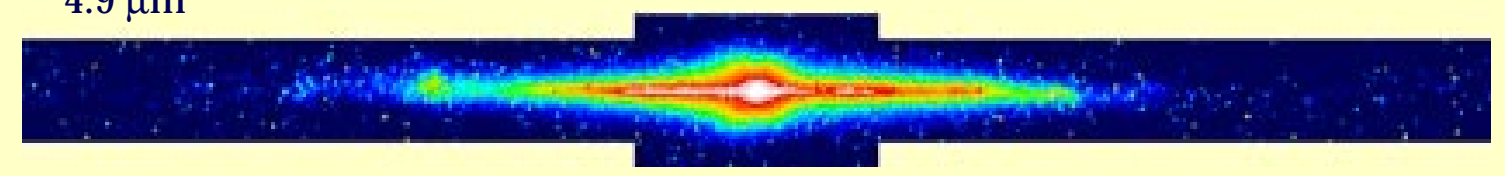

Min

$\operatorname{Max}$

Fig. 72. DIRBE maps of sky brightness at $1.25,2.2,3.5$, and $4.9 \mu \mathrm{m}$ at low Galactic latitudes $(|b| \leq 15$ deg within 30 degrees of Galactic center, and $|b| \leq 10 \mathrm{deg}$ elsewhere). Zodiacal light has been removed. North is up, and galactic longitude is increasing from right to left. These maps are generally dominated by Galactic starlight. No extinction correction has been made. Intensities are provided at 16 levels on logarithmic scales ranging from 0.6 to $25 \mathrm{MJy} / \mathrm{sr}(1.25 \mu \mathrm{m}$ and $2.2 \mu \mathrm{m}), 0.4$ to $16 \mathrm{MJy} / \mathrm{sr}(3.5 \mu \mathrm{m})$, and 0.3 to $12.5 \mathrm{MJy} / \mathrm{sr}(4.9 \mu \mathrm{m})$. In detail these levels are: $0.63,0.81,1.03,1.32,1.69,2.15,2.75,3.52,4.50,5.75,7.36,9.40$, $12.02,15.37,19.65$, and $25.12 \mathrm{MJy} / \mathrm{sr}$ at $1.25 \mu \mathrm{m}$ and $2.2 \mu \mathrm{m} ; 0.40,0.51,0.65,0.83,1.06,1.36,1.74,2.22,2.84,3.63,4.64,5.93$, $7.59,9.70,12.40$, and $15.85 \mathrm{MJy} / \mathrm{sr}$ at $3.5 \mu \mathrm{m} ; 0.32,0.40,0.52,0.66,0.84,1.08,1.38,1.76,2.26,2.88,3.69,4.71,6.03,7.70,9.85$, and $12.59 \mathrm{MJy} / \mathrm{sr}$ at $4.9 \mu \mathrm{m}$

from zodiacal light are negligible (Hanner et al. 1974). The instantaneous field of view of the Pioneer 10 photometer was $2.28^{\circ}$ square, which due to spacecraft spin $(12.5 \mathrm{~s}$ period) and finite integration time $(0.2 \mathrm{~s})$ was drawn into elongated effective fields-of-view of variable size depending on the look angle. Contributions due to unresolved stars begin to enter the Pioneer 10 data at $m \geq 6.5$ for an average galactic latitude; thus, stars dominate the measured fluxes.

Toller (1981) derived DGL intensities from the Pioneer 10 blue data (440 $\mathrm{nm}$ ) by subtracting integrated starlight intensities of Roach \& Megill (1961) and Sharov \& Lipaeva (1973) at the positions of 194 Selected Areas (Blaauw \& Elvius 1965). The residuals, interpreted as the sum of DGL and extragalactic background light, are most representative in terms of sky coverage.

For reference purposes, several directions may be taken to estimate the intensity of the DGL at $\lambda \approx 440 \mathrm{~nm}$ :
In Fig. 76 we present the mean galactic latitude dependence of Toller's (1981) values of the sum of DGL and extragalactic background, averaged over all galactic longitudes. The error bars, representing one standard deviation of the mean, reflect in part the real variations of the DGL intensity with galactic longitude, especially at lower latitudes.

A second avenue toward a DGL estimate can be found in ratios of DGL to total line-of-sight starlight $\left(\mathrm{LOS}_{*}\right)$ intensities. In Table 39 we list the average ratios

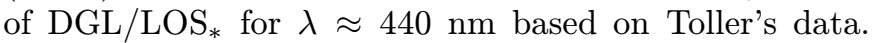
The use of the values in Table 39 may be advisable, if one wants to estimate the variation of DGL with galactic longitude, where large differences in $\mathrm{LOS}_{*}$ may occur. Due to the strongly forward scattering nature of interstellar grains the DGL intensity generally tracks the $\mathrm{LOS}_{*}$ intensity at constant latitude. 

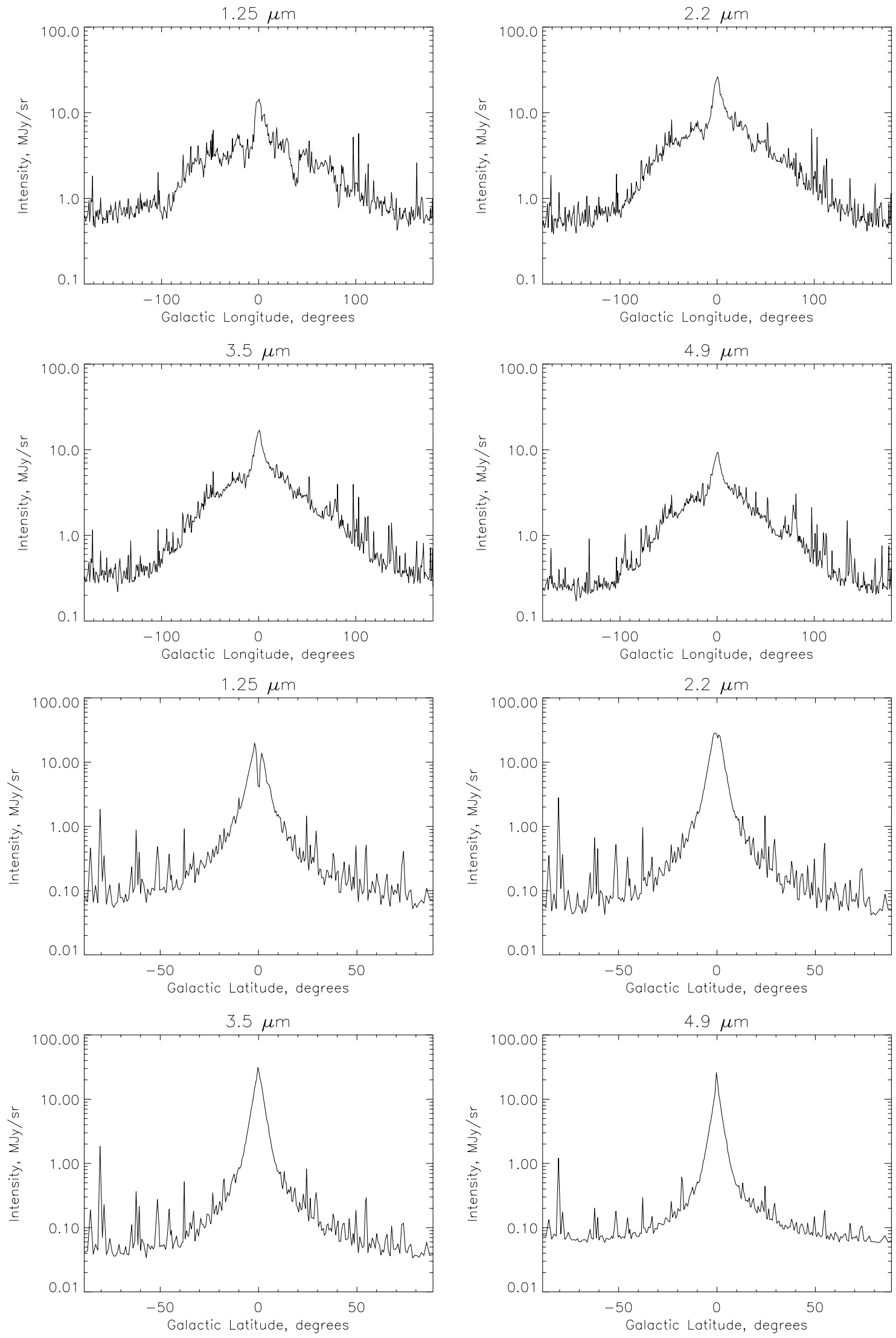

Fig. 73. Intensity profiles of "Galactic starlight" as measured from the DIRBE maps at $1.25 \mu \mathrm{m}, 2.2 \mu \mathrm{m}, 3.5 \mu \mathrm{m}$ and $4.9 \mu \mathrm{m}$ after subtraction of zodiacal light. Upper half: longitudinal profiles at a fixed Galactic latitude of $b=1.6 \mathrm{deg}(b=0 \mathrm{deg}$ is not shown as representative because extinction is significant at some wavelengths). Lower half: latitudinal profiles at fixed Galactic longitude of $l=0 \mathrm{deg}$ 


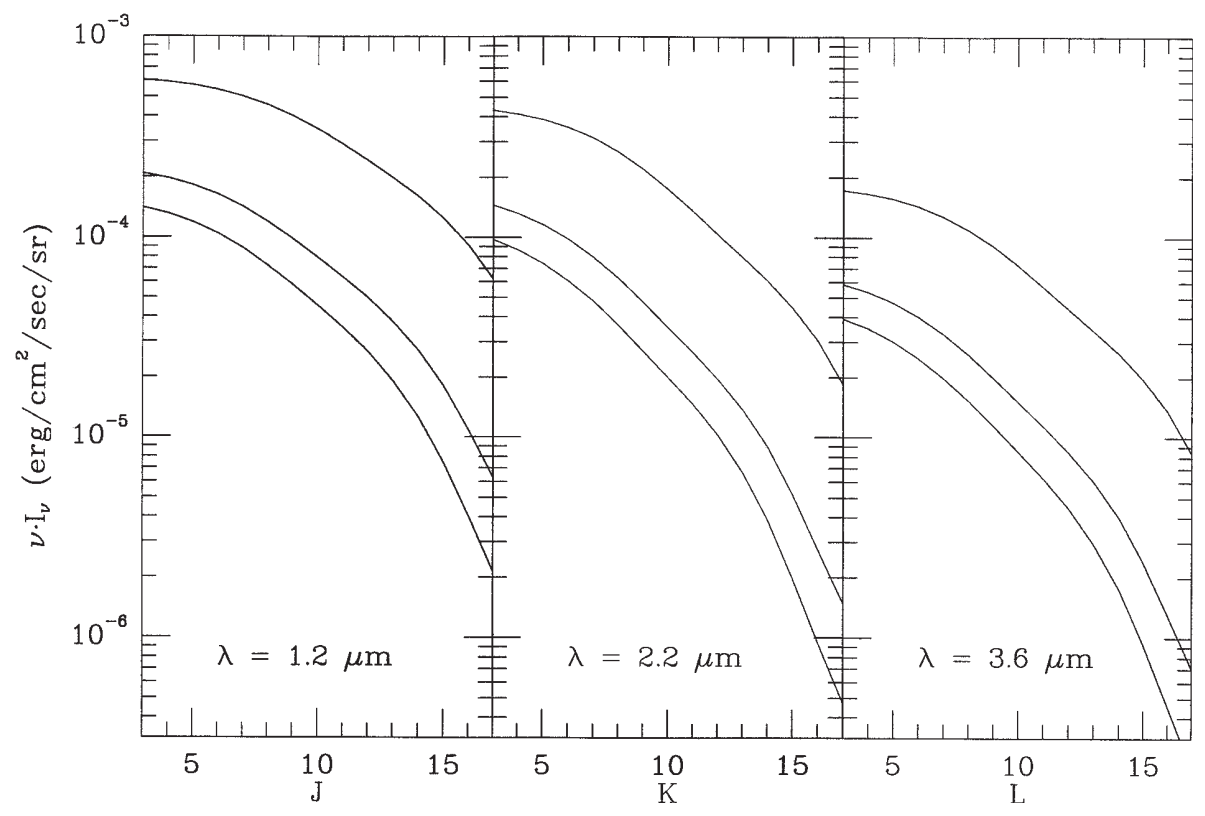

Fig. 74. Residual contributions to the near-infrared background radiation of stars fainter than a given apparent magnitude, for galactic latitudes of $20^{\circ}, 50^{\circ}$, and $90^{\circ}$ (from top to bottom, respectively). The values at the galactic pole at the intersection with the ordinate axis (cutoff magnitude $=3 \mathrm{mag}$ ) corresponds to $0.063 \mathrm{MJy} / \mathrm{sr}$ for $J$, to $0.081 \mathrm{MJy} / \mathrm{sr}$ for $K$, and to $0.053 \mathrm{MJy} / \mathrm{sr}$ for the $L$ waveband

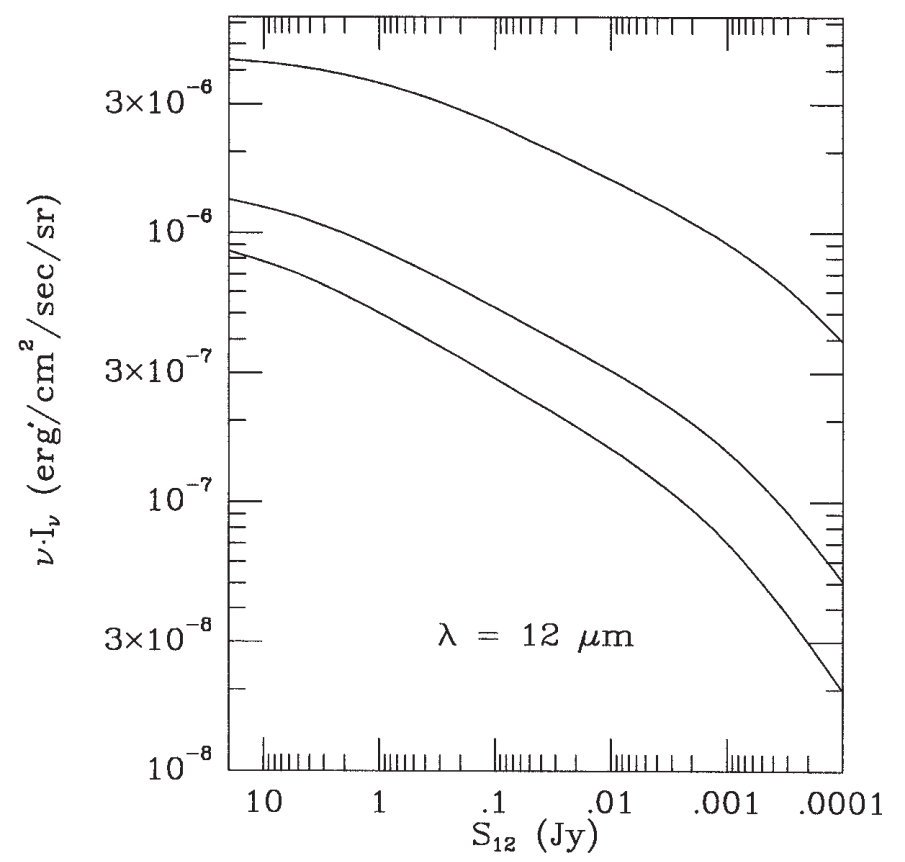

Fig. 75. Residual contributions to the $12 \mu \mathrm{m}$ background radiation of stars fainter than a given flux limit, for galactic latitudes of $20^{\circ}, 50^{\circ}$, and $90^{\circ}$ (from top to bottom, respectively). The values at the galactic pole at the intersection with the ordinate axis (cutoff flux $=20 \mathrm{Jy}$ ) correspond to $0.002 \mathrm{MJy} / \mathrm{sr}$, which is much less than the contribution due to diffuse emission from the interstellar medium

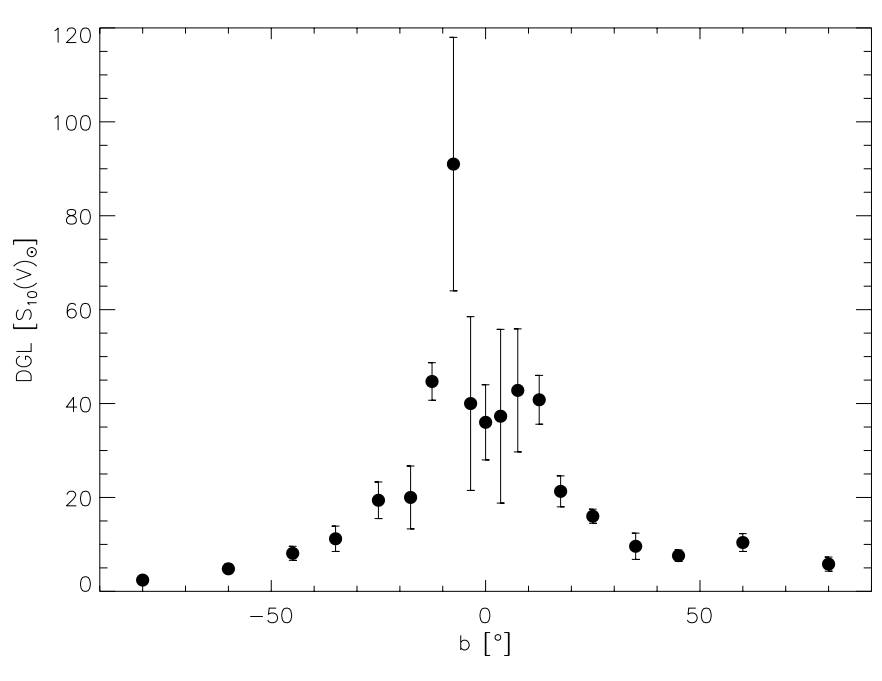

Fig. 76. The average intensity of the DGL as a function of galactic latitude based on the analysis by Toller (1981) of Pioneer 10 photometry of 194 Selected Areas at $\lambda_{\text {eff }}=440 \mathrm{~nm}$. Error bars denoting one standard deviation of the means are a measure of the longitudinal variation of the DGL intensity

A third approach toward a DGL estimate might rely on the mean correlation between DGL intensities found in Selected Areas by Toller (1981) and corresponding column densities of atomic hydrogen. Toller finds:

$$
\operatorname{DGL}\left(S_{10}(V) \odot\right)=N_{\mathrm{HI}} /\left(2.410^{20}\left[\text { atoms } \mathrm{cm}^{-2}\right]\right) .
$$


Table 39. Ratio of DGL to line-of-sight starlight

\begin{tabular}{ccc}
\hline$b\left[^{\circ}\right]-N[\mathrm{SA}]$ & $\left\langle\mathrm{DGL} / \mathrm{LOS}_{*}\right\rangle$ & \\
\hline $0-|5|$ & 19 & $0.21 \pm 0.05$ \\
$|5|-|10|$ & 11 & $0.34 \pm 0.07$ \\
$|10|-|15|$ & 28 & $0.31 \pm 0.03$ \\
$|15|-|20|$ & 15 & $0.19 \pm 0.04$ \\
$|20|-|30|$ & 29 & $0.25 \pm 0.04$ \\
$|30|-|40|$ & 22 & $0.17 \pm 0.04$ \\
$|40|-|60|$ & 41 & $0.17 \pm 0.02$ \\
$|60|-|90|$ & 29 & $0.12 \pm 0.02$ \\
\hline
\end{tabular}

A good source for $N_{\mathrm{HI}}$ values is the Bell Lab HI survey by Stark et al. (1992). This approach is based on the fact that the dust and HI column densities are well-correlated (Bohlin et al. 1978) and that the DGL intensity is determined in part by the dust column density, as long as the line-of-sight is not optically thick. This third approach is therefore recommended mostly for higher galactic latitudes, or $N_{\mathrm{HI}}<210^{21}$ atoms $\mathrm{cm}^{-2}$. Estimates based on Eq. (31) are at best good to within a factor of two, because Eq. (31) reflects only the dependence of the DGL intensity on the dust column density and ignores the dependence on the intensity of the illuminating radiation field.

The red band ( $\lambda=640 \mathrm{~nm}$ ) data from Pioneer 10 have not been subjected to a DGL analysis so far for lack of suitable star count data.

The $U-B$ and $B-V$ colours of the DGL have been measured and have been found to be bluer than the colour of the integrated starlight, as expected from scattering by interstellar grains with scattering cross sections varying as $\lambda^{-1}$ in the visible region (Witt 1968; Mattila 1970). Table 40, to give an example, contains $U B V$ colours of the DGL and of the integrated starlight in Cygnus (upper panel), respectively in Crux (lower panel).

Table 40. Colour of the DGL

\begin{tabular}{cccccl}
\hline \multicolumn{4}{c}{ DGL } & \multicolumn{4}{c}{ Integrated Starlight Reference } \\
$l,|b|$ & $U-B$ & $B-V$ & $U-B$ & $B-V$ & \\
\hline $70^{\circ}, 0^{\circ}$ & -0.05 & +0.57 & +0.07 & +0.73 & Witt \\
$75^{\circ}, 25^{\circ}$ & -0.10 & +0.44 & +0.00 & +0.68 & $(1968)$ \\
\hline $300^{\circ}, 0^{\circ}$ & -0.10 & +0.50 & -0.01 & +0.71 & Mattila \\
& & & & $(1970)$ \\
\hline
\end{tabular}

Recently, Gordon (1997) reported the detection of extended red emission (ERE) on a galaxy-wide scale in the diffuse interstellar medium of the Milky Way Galaxy (see also Gordon et al. 1997; Gordon \& Witt 1997). The ERE consists of a broad emission band (FWHM $800 \AA$ ) with a peak wavelength found in the $6500 \AA$ to $8000 \AA$ range, depending on environment, with a long-wavelength tail extending well into the $I$-band. The ERE is believed to result from a photoluminescence process in hydrogenated carbonaceous grain mantles, and it has been previously detected photometrically and spectroscopically in numerous reflection nebulae (Witt \& Schild 1988; Witt \& Boroson 1990), in carbon-rich planetary nebulae (Furton \& Witt 1990, 1992), in HII regions (Perrin \& Sivan 1992; Sivan \& Perrin 1993), and in the scattered light halo of the starburst galaxy M 82 (Perrin et al. 1995). Gordon (1997) derived the galactic ERE intensity from Pioneer 10 and 11 sky photometry obtained at heliocentric distances greater than 3.3 AU, where the contribution from zodiacal light is no longer detectable (see Sect. 10.4). The integrated star light due to stars of $m>6.5$ was determined by integrating recent starcount data from the APS Catalog (Pennington et al. 1993), the HST Guide Star Catalog, and photometric catalogs on brighter stars and was subtracted from the Pioneer 10 and 11 in both the blue and red bands. The diffuse residuals consist of DGL in the blue band, and of a sum of DGL and ERE in the red band. As a result, the $B-R$ colour of the diffuse galactic background radiation is substantially redder than that of the DGL alone. The excess ERE in the $R$-band can be estimated to be about equal in intensity to the $R$-band DGL. This ERE intensity is consistent with the measured $B-R$ and $B-I$ colour excesses of individual galactic cirrus filaments (Guhathakurta \& Tyson 1989), found to be $0.5-1.0 \mathrm{mag}$ and 1.5 - 2.0 mag redder, respectively, than expected for scattered disk starlight.

Quantitatively, Gordon (1997) finds the galactic ERE and the atomic hydrogen column density at intermediate and high latitudes $\left(|b|>20^{\circ}\right)$ to be wellcorrelated, yielding an average ERE intensity of (1.43 \pm 0.31) $10^{-29} \mathrm{erg} \mathrm{s}^{-1} \AA^{-1} \mathrm{sr}^{-1} \mathrm{H}$-atom ${ }^{-1}$. This correlation may therefore be used to estimate the expected ERE intensity in the $R$-band in different portions of the sky.

Partial linear polarization of the DGL at a level of $1-2 \%$ is expected, and some tentative detections of this polarization have been reported by Schmidt \& Leinert (1966), Weinberg (1969), Sparrow \& Ney (1972), and Bandermann \& Wolstencroft (1976). Both the scattering by grains partially aligned with their short axes parallel to the galactic plane and the scattering of the non-isotropic galactic radiation field by dust in the galactic plane should produce partially polarized scattered light with the electric vector perpendicular to the galactic plane when observed near $b=0^{\circ}$. A review of existing polarization measurements is given by Leinert (1990).

\subsection{Near-infrared}

The diffuse galactic background radiation in the nearinfrared (near-IR) is composed of several components, 
each produced by different constituents of the diffuse interstellar medium by different physical processes. The most important ones are the DGL, caused by scattering of star light on larger interstellar grains; the near-IR continuum emission, caused by a non-equilibrium emission process probably associated with small carbonaceous grains; and the set of so-called unidentified infrared bands which have been attributed to emission from interstellar aromatic hydrocarbon molecules, such as polycyclic aromatic hydrocarbons $(\mathrm{PAH})$. We will refer to them as aromatic hydrocarbon bands.

No separate detection of the DGL at near-IR wavelengths has been accomplished so far, although the galactic component of the near-IR background at $1.25 \mu \mathrm{m}$ and $2.2 \mu \mathrm{m}$ observed by the DIRBE experiment (Silverberg et al. 1993; Hauser 1996) undoubtedly contains a scattered light contribution. Recent evidence (Witt et al. 1994; Lehtinen \& Mattila 1996) provides a strong indication that the dust albedo remains as high as it is in the visible out through the $K$-band $(2.2 \mu \mathrm{m})$. The $K$-optical depth is about $10 \%$ of that at $V$; hence, only at quite low galactic latitudes $\left(|b|<5^{\circ}\right)$ can one find the required dust column densities which will give rise to substantial (scattered) DGL. At the galactic equator, however, the ratio of $\mathrm{DGL} / \mathrm{LOS}_{*}$ should be similar to the values listed in Table 39. At higher galactic latitudes the ratio DGL/LOS * will be substantially lower than the values listed in Table 39.

The near-IR continuum emission was first recognized in reflection nebulae whose surface brightnesses in the $1 \mu \mathrm{m}-10 \mu \mathrm{m}$ wavelength range exceeded that expected from scattering by factors of several (Sellgren et al. 1983; Sellgren 1984). Absence of polarization provided additional confirmation of the non-scattering origin of this radiation. The non-equilibrium nature of the radiation process was recognized from the fact that the colour temperature of the emerging radiation was independent of distance from the exciting star and thus independent of the density of the exciting radiation. This leaves as the cause of this radiation non-equilibrium processes which depend upon excitation by single photons, e.g. photoluminescence of grain mantles or, alternatively, non-equilibrium heating of tiny grains resulting in large temperature fluctuations. The galactic distribution of this radiation component has yet to be studied; it depends on a very accurate assessment of the near-IR integrated starlight (see Sect. 10.5) and the near-IR zodiacal light (see Sect. 8.5), which need to be subtracted from photometries of the near-IR sky background.

The aromatic hydrocarbon bands centered at wavelengths $3.3 \mu \mathrm{m}, 6.2 \mu \mathrm{m}, 7.7 \mu \mathrm{m}, 8.6 \mu \mathrm{m}$, and $11.3 \mu \mathrm{m}$, with widths in the range of 0.03 to $0.5 \mu \mathrm{m}$, were first observed in bright nebulous regions by Gillett et al. (1973). Thanks to the successful AROME balloon-borne experiment (Giard et al. 1988; Ristorcelli et al. 1994) and the more recent missions of the Infrared Telescope in Space (IRTS, Onaka et al. 1996) and the Infrared Space Observatory (ISO, Mattila et al. 1996; Lemke et al. 1997), they have now been observed in the diffuse interstellar medium at low galactic latitudes. The relative bandstrengths and widths are very similar to those observed in reflection nebulae, planetary nebulae, and HII regions, pointing toward a common emission mechanism. Onaka et al. (1996) show that the band intensities at $3.3 \mu \mathrm{m}$ and $7.7 \mu \mathrm{m}$ and the far-IR background intensities at $100 \mu \mathrm{m}$ along identical lines of sight are correlated very tightly, suggesting that the respective emitters, presumably PAH molecules in the case of the aromatic hydrocarbon bands and classical sub-micron grains for the $100-\mu \mathrm{m}$ thermal continuum, are well-mixed spatially and are excited by the same interstellar radiation field. The correlation of the band intensities with the atomic hydrogen column density is also excellent, reflected in the dust emission spectrum per hydrogen atom given in Table 43.

\subsection{Thermal infrared}

The infrared emission from the diffuse galactic ISM is dominated by thermal and other emissions by dust, with some additional contributions from interstellar cooling lines, mainly from CII and NII. At wavelengths $<100 \mu \mathrm{m}$ the galactic diffuse emission is weaker than the infrared emission from the zodiacal dust cloud (see Fig. 1 ); at wavelengths $>400 \mu \mathrm{m}$ the cosmic background radiation dominates over the galactic thermal radiation. Only in the $100-400 \mu \mathrm{m}$ band is the galactic emission the primary background component. However, as the composite spectrum of all night sky components in Fig. 1 schematically indicates, the thermal IR spectrum of galactic dust is complex in structure, suggesting significant contributions from grains covering a wide range of temperatures. In particular, there is substantial excess emission in the 5 to $50 \mu \mathrm{m}$ spectral range. This excess is generally attributed to stochastically heated very small grains with mean temperatures in the range $100-500 \mathrm{~K}$ (Draine \& Anderson 1985; Weiland et al. 1986), while the main thermal emission peak near $150 \mu \mathrm{m}$ is attributed to classical-sized dust grains in equilibrium with the galactic interstellar radiation field, resulting in temperatures around $20 \mathrm{~K}$.

The exploration of the infrared background has been greatly advanced by the highly successful missions of the Infrared Astronomical Satellite (IRAS; Neugebauer et al. 1984), the Diffuse Infrared Background Experiment (DIRBE; Boggess et al. 1992) and the Far-Infrared Absolute Spectrophotometer (FIRAS; Fixsen et al. 1994) on board of the COBE satellite, the Infrared Telescope in Space (IRTS; Murakami et al. 1994, 1996), and the AROME balloon-borne experiment (Giard et al. 1988). Before mentioning the relation to interstellar gas, we first comment on the maps of galactic far-infrared emission available from these experiments. 
From IRAS, the so-called ISSA maps are available for wavelengths of $12 \mu \mathrm{m}, 25 \mu \mathrm{m}, 60 \mu \mathrm{m}$ and $100 \mu \mathrm{m}$. These present, after subtraction of a zodiacal light model, $12.5^{\circ} \times$ $12.5^{\circ}$ fields with $1.5^{\prime}$ resolution, covering the sky on a $10^{\circ}$ grid. For the two longer wavelengths this gives a good picture of the variation of galactic emission (in MJy/sr). The absolute value of these maps is not reliable, which can be seen from the regions with negative intensities. To access the data one can use the world wide web address http://www.ipac.caltech.edu/ipac/iras/released_data.html.

With better modeling of the zodiacal light contribution, Rowan-Robinson et al. (1991) produced sky maps of galactic plus extragalactic far-infrared emission with $0.5^{\circ}$ pixel size. These maps give realistic absolute values and still show the spatial variation of the galactic diffuse emission in some detail. Tables 41 and 42 give a version of the two tables for $60 \mu \mathrm{m}$ and $100 \mu \mathrm{m}$ in ecliptic coordinates. They are not printed here but available in electronic form at the CDS by anonymous ftp to 130.79.128.5.

But in particular the DIRBE Zodiacal LightSubtracted Mission Average ("ZSMA") maps at $60 \mu \mathrm{m}$, $100 \mu \mathrm{m}, 140 \mu \mathrm{m}$ and $240 \mu \mathrm{m}$ give a good estimate of the observed intensity resulting from the sum of galactic diffuse infrared emission and the extragalactic background at each of these wavelengths, apart from errors in the zodiacal light model model. Figure 77 shows the DIRBE 240 micron ZSMA map, along with representative intensity profiles at fixed galactic latitude. Since the extragalactic background light, which is spatially uniform, is not necessarily negligible at these wavelengths (see Table 47 in Sect. 12), caution should be exercised when making quantitative statements about the absolute level of the diffuse Galactic infrared emission as derived from these maps. The Galactic contribution is certainly dominant at latitudes $|b| \leq 10^{\circ}$, but probably also all over the sky. Aside from this issue of absolute levels (how much extragalactic background light radiation exists and has to be subtracted?), and aside from some visible zodiacal light model artifacts in the ecliptic plane at 60 microns, the ZSMA maps give a good picture of the spatial variation of the diffuse Galactic emission at all latitudes. The intensities recorded in the ZSMA maps are reported in $\mathrm{MJy} / \mathrm{sr}$, assuming a nominal wavelength and a spectral shape of $\nu I_{\nu}=$ constant. The ZSMA maps are available from the NSSDC through the COBE homepage website at http://www.gsfc.nasa.gov/astro/cobe/cobe_home.html.

Interstellar dust appears to be well-mixed with all phases of the interstellar gas (Boulanger \& Perault 1988; Sodroski et al. 1997); however, to obtain a first-order representation of the emissions from galactic dust, the well-established correlations with $N(\mathrm{HI})$ provide the best guide. The average dust emission spectrum per $\mathrm{H}$-atom is given in Table 43, as derived from the following original sources: ERE at $0.65 \mu \mathrm{m}$, Gordon (1997); galactic emission in the $3.3 \mu \mathrm{m}$ aromatic feature, Giard et al. (1989), Bernard et al. (1994); dust emission in the 6.2,
7.7, 8.6, and $11.3 \mu \mathrm{m}$ mid-infrared unidentified bands, Onaka et al. (1996); and the broad-band thermal dust emissions, Boulanger et al. (1996), Reach et al. (1995a), and Dwek et al. (1997).

Table 43. Dust emission spectrum per hydrogen atom in the average interstellar medium

\begin{tabular}{cc}
\hline Wavelength & $\begin{array}{c}\text { Dust emission spectrum } \\
4 \pi \lambda I_{\lambda}\end{array}$ \\
$(\mu \mathrm{m})$ & $\left(\mathrm{erg} \mathrm{s}^{-1} \mathrm{H}^{\left.- \text {atom }^{-1}\right)}\right.$ \\
\hline 0.65 & $1.1710^{-24}$ \\
3.5 & $1.270^{-25}$ \\
4.9 & $1.3610^{-25}$ \\
7.7 & $1.1310^{-25}$ \\
12 & $1.021^{-24}$ \\
25 & $4.1110^{-25}$ \\
60 & $7.0410^{-25}$ \\
100 & $2.6410^{-24}$ \\
140 & $3.6410^{-24}$ \\
240 & $1.410^{-24}$ \\
346 & $5.7710^{-25}$ \\
490 & $1.770^{-25}$ \\
535 & $1.2010^{-25}$ \\
736 & $3.3310^{-26}$ \\
1100 & $5.4910^{-27}$ \\
\hline
\end{tabular}

Compared with emission from dust, the radiation from infrared cooling lines of the gas is comparatively weak, reflecting the fact that dust in interstellar space absorbs approximately one third of all energy emitted by stellar sources. The three strongest lines are the [CII] transition at $158 \mu \mathrm{m}$ and the [NII] lines at 122 and $205 \mu \mathrm{m}$ (Wright et al. 1991). At low galactic latitudes, the [CII] line emission is well correlated with $N(\mathrm{HI})$, yielding $(2.65 \pm$ 0.15) $10^{-26} \mathrm{ergs} \mathrm{s}^{-1} \mathrm{H}_{\text {-atom }}{ }^{-1}$ (Bennett et al. 1994). With latitudes increasing beyond $|b|=20^{\circ}$, the ratio of $I([158]) / I\left(\right.$ FIR $\left._{\text {continuum }}\right)$ decreases rapidly, leaving the $158 \mu \mathrm{m}$ line unmeasurably weak at $|b|>65^{\circ}$.

\subsection{Ultraviolet}

11.5.1. FUV diffuse galactic light (91.2 nm to $180 \mathrm{~nm}$ )

Intense Lyman $\alpha$ flux is present in this bandpass, and this, combined with various instrumental limitations, meant that most of the effort to measure a diffuse flux in this band was carried out at wavelengths longer than $121.6 \mathrm{~nm}$. In retrospect, the measurement of a diffuse flux in this bandpass is far more difficult than was originally imagined, and the potential for obtaining erroneous results is substantial. The literature is filled with controversial and erroneous results. 


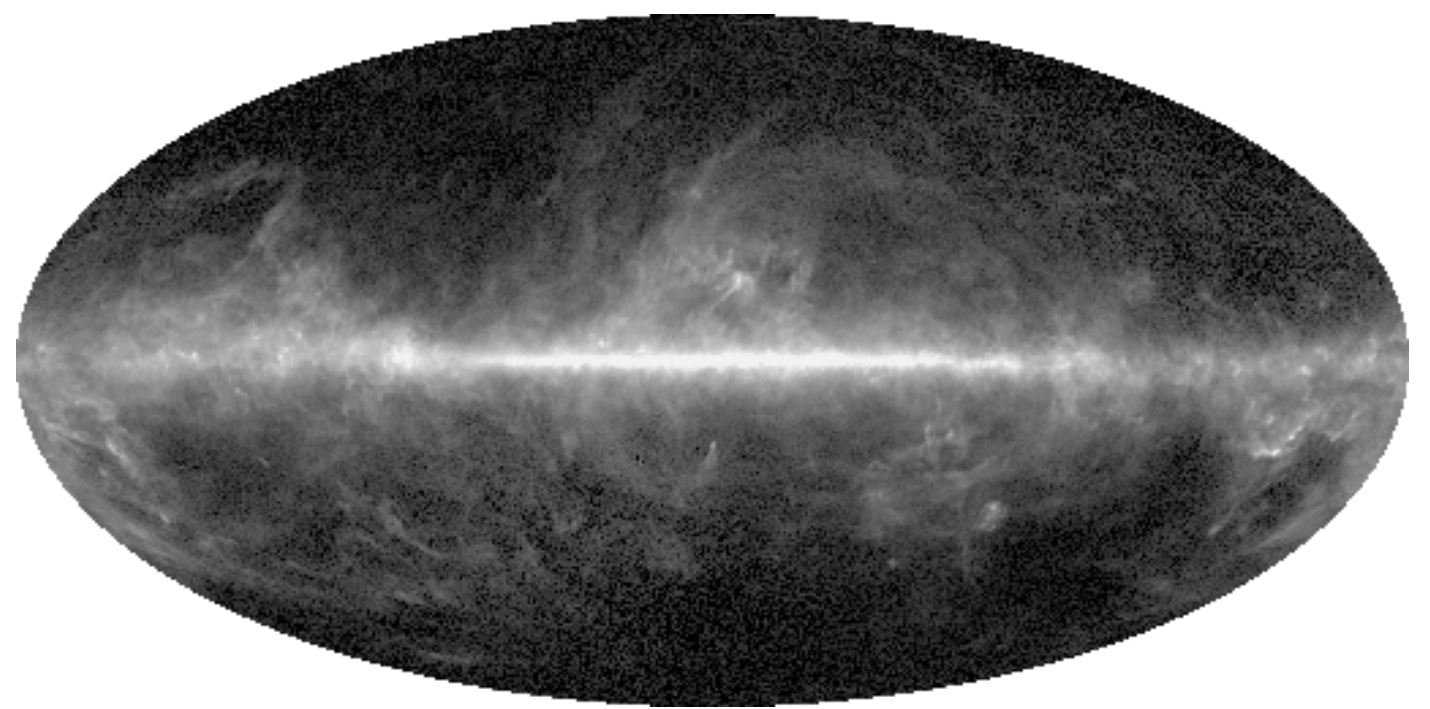

1.

1000.
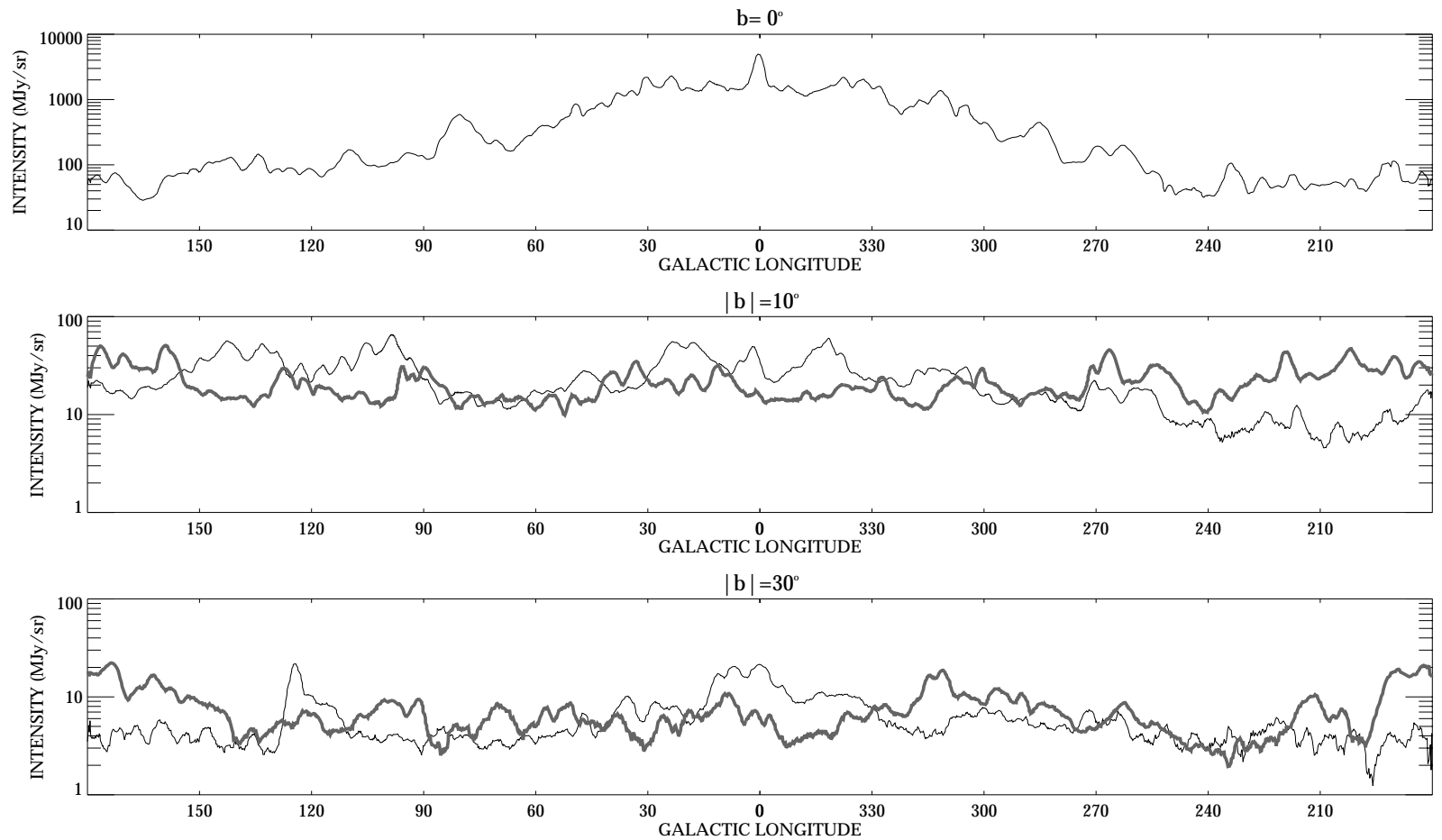

$|\mathrm{b}|=60^{\circ}$

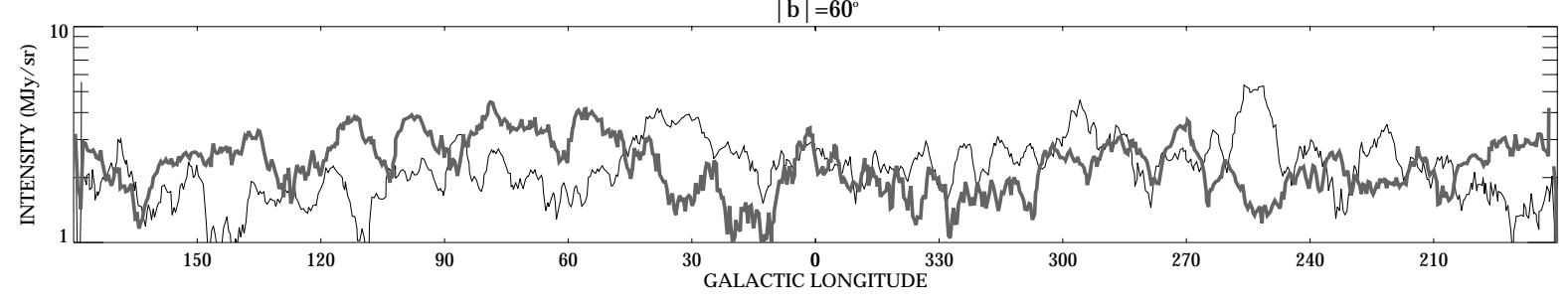

Fig. 77. Map of the sky brightness at $240 \mu \mathrm{m}$ after removal of zodiacal light, obtained from the COBE/DIRBE experiment. The map is a Mollweide projection in Galactic coordinates, with intensities on a logarithmic stretch from 1 to $1000 \mathrm{MJy} / \mathrm{sr}$. Smoothed intensity profiles at fixed Galactic latitudes of $0^{\circ},+/-10^{\circ},+/-30^{\circ}$ and $+/-60^{\circ}$ are also plotted; positive latitudes are represented by the thin black, negative latitudes by the thick grey line 


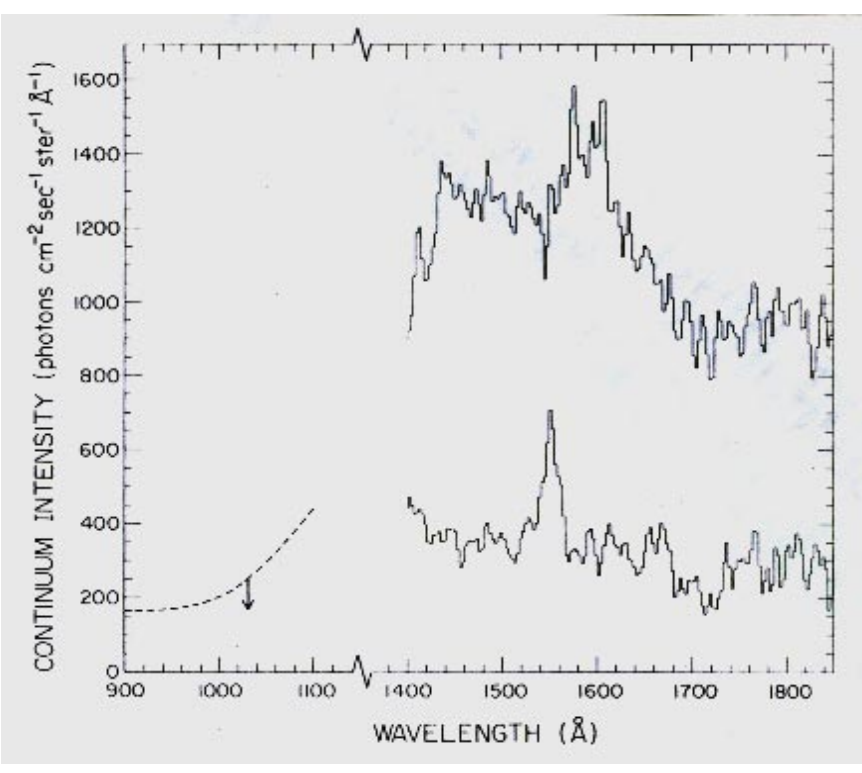

Fig. 78. Summary data on the diffuse cosmic far ultraviolet background. The data from 902 to $1200 \AA$ are from Holberg (1986) and are upper limits to the flux from a high Galactic latitude view direction. Two data sets are shown for the 1400 to $1850 \AA$ band. The upper line is from Hurwitz et al. (1991) and shows typical data obtained in view directions with $\tau_{\text {dust }} \geq$ 1. Note the $\mathrm{H}_{2}$ fluorescence emission around $160 \mathrm{~nm}$. The lower line is from Martin \& Bowyer (1990) and shows data obtained at a high Galactic latitude; the CIV $1550 \AA$ line is clearly evident in emission and the $1663 \AA$ line of OIII] is also apparent, though at lower signal-to-noise ratio. The extragalactic contribution to these data probably is small (see Table 44)

This bandpass has been studied extensively since the beginning of the Space Age, because the zodiacal light component is not present and contributions from stellar sources were expected to be sufficiently low that emission from a hot $\left(10^{5} \mathrm{~K}\right)$ or very hot $\left(10^{6} \mathrm{~K}\right)$ intergalactic medium might be detected.

A review of this literature is provided by Bowyer (1991), but for an alternative view and a detailed examination of an particular data set, see Henry (1991).

Substantial progress has been made in identifying the components that contribute to the diffuse flux in this band. The vast majority of the diffuse flux is starlight scattered by interstellar dust. In particular, Haikala et al. (1995) found FUV emission at a high galactic latitude from a cirrus cloud detected at $100 \mu \mathrm{m}$ with IRAS. Emission from hot $\left(\approx 10^{5} \mathrm{~K}\right)$ gas has been detected. An analysis of this radiation establishes that the emitting gas is well above the Galactic plane. Two-photon emission from recombining ionized hydrogen has been recognized as a component of this background. Molecular hydrogen fluorescence has been found in low density clouds. Any ex-
Table 44. Components of the diffuse cosmic far ultraviolet background with approximate intensities ${ }^{a}$ (photons $\mathrm{cm}^{-2} \mathrm{~s}^{-1}$ $\left.\mathrm{sr}^{-1} \AA^{-1}\right)$

\begin{tabular}{lc}
\hline Total intensity & $300-1500$ \\
Scattering by dust & $200-1500$ \\
H II two-photon emission & 50 \\
H2 fluorescence & 100 \\
(in molecular clouds) & 10 \\
$\begin{array}{l}\text { Hot gas line emission } \\
\text { from hot Galactic gas }\end{array}$ & 50 to 200 \\
Extragalactic & none to 200 \\
Unexplained & \\
\hline
\end{tabular}

\section{${ }^{a}$ Intensities dependent upon view direction.}

Intensities of processes producing discrete features are averaged over the 1400-1859 A band.

tragalactic flux is quite small; this component is discussed in Sect. 12.1.

In Fig. 78 we provide examples of the best available data on the diffuse far ultraviolet background. The data from 912 to $\approx 1200$ Angstrom are from Holberg (1986) and are upper limits to the background from a high galactic latitude view direction. Two data sets are shown for the 1400 to 1840 Angstrom band. The upper line is from Hurwitz et al. (1991) and shows data obtained at a low galactic latitude. These data are typical of what is observed in viewing directions with an optical depth of $\tau_{\text {dust }} \geq 1$. Molecular hydrogen fluorescence is evident as an additional component at wavelengths from 1550 to 1650 Angstrom. The lower line is from Martin \& Bowyer (1990) and shows data obtained at a high galactic latitude and a low total galactic neutral hydrogen column. The CIV 1550 Angstrom line is clearly evident in emission, and the 1663 Angstrom line of forbidden O III is also apparent though at a lower signal-to-noise.

As already mentioned, the major components of the cosmic far ultraviolet background are summarized in Table 44 above.

\subsubsection{Near-ultraviolet $(180 \mathrm{~nm}-300 \mathrm{~nm})$}

The diffuse radiation in this band is the sum of zodiacal light and starlight scattered by interstellar dust. A few first studies of the zodiacal light in this band have been carried out, which suggest this component exhibits characteristics similar to that observed in the visible (see Sect. 8.6). A few studies of scattering by dust by early type stars have been carried out. The results obtained differ, and independent of these differences, the scattering varies tremendously from place to place in the galaxy. We refer the reader to Dring et al. (1996) and references therein for a discussion of these results. 


\section{Extragalactic background light}

For the extragalactic background radiation no generallyaccepted measured values exist in the UV, optical or infrared wavebands. However, upper limits from surface photometry and lower limits from galaxy counts are available. We present a critical evaluation and tabulation of the available results.

Extragalactic background light (EBL) in UV, optical and near-IR $(\lambda \lesssim 5 \mu \mathrm{m})$ is thought to consist mainly of redshifted starlight from unresolved galaxies; more hypothetical contributions would be, e.g., from stars or gas in the intergalactic space, and from decaying elementary particles (e.g. neutrinos). In the mid- and far-infrared the main contribution is thought to be redshifted emission from dust particles, heated by starlight in galaxies.

Observations of the EBL are hampered by the much stronger foreground components of the night sky brightness described in the other sections. Unlike the other components the EBL is isotropic which, in combination with its weakness, complicates its separation. Recent reviews of the observational and theoretical status of the EBL have been given by Mattila (1990), Tyson (1990, 1995), Mattila et al. (1991) for the optical; by Bowyer (1991), Henry (1991), Henry \& Murthy (1995) and Jakobsen (1995) for the ultraviolet; by Matsumoto (1990), Franceschini et al. (1991a), Hauser (1995a,b, 1996) and Lonsdale (1995) for the infrared; Longair (1995) has given a general review covering all wavelengths.

The observational results presented here are summarised for each wavelength range in a separate table in the corresponding subsection. They are also put together in overview in Fig. 79 at the end of this section, where in the visual and near-infrared region some model predictions are added for comparison with the data, which stretch over a wide range of brightnesses at these wavelengths. Otherwise, model prediction of EBL brightness are not the topic of this reference. For this matter see, e.g., the conference proceedings by Bowyer \& Leinert (1990) and RoccaVolmerange et al. (1991) or the work of Franceschini et al. (1991b).

\subsection{Ultraviolet}

An extragalactic component is certainly present in the $\mathrm{UV} / \mathrm{FUV}$ since the summed flux of galaxies is present at some level. Early in the Space Age it was realized that searches in the FUV had substantial advantages over searches in the UV, because the zodiacal light component is not present at a measurable level and contributions from stellar sources were expected to be small. In particular, it was hoped that emission from a very hot $\left(10^{6} \mathrm{~K}\right)$ or hot $\left(10^{5} \mathrm{~K}\right)$ intergalactic medium might be detected. These measurements were far more difficult to carry out than was originally anticipated, and a wide range of conflicting results were reported.
At this point, the most cited argument that some of the Far UV diffuse background is extragalactic in origin is that most measurements of this flux show a correlation with galactic neutral hydrogen column density, and the extrapolation to zero hydrogen columns yields fluxes that are in the range of 50 to 300 photon units. These results are only upper limits to an extragalactic background, however, since there is no guarantee that galactic components are not producing this flux.

While the total picture is far from clear, some aspects of a possible extragalactic flux have been established. Quasar absorption line studies definitely constrain emission from a diffuse intergalactic medium to a marginal role (Jakobsen 1991). Paresce \& Jacobsen (1980) had shown before that integrated light from QSOs and AGNs will not produce a significant contribution to the diffuse FUV background. However, Armand et al. (1994) have used data on galaxy counts obtained at $2000 \AA$ with a limiting magnitude of 18.5 , to calculate the ultraviolet flux due to the integrated FUV light of all galaxies. The extrapolation is small and leads to an expected flux of 40 to 130 photon $\mathrm{cm}^{-2} \mathrm{~s}^{-1} \mathrm{sr}^{-1} \AA^{-1}$. Hence it seems certain that there is at least some extragalactic flux present in the diffuse FUV background. It is interesting to note that the flux predicted by Armand et al. is consistent with the (uncertain and controversial) observational results for a possible extragalactic diffuse FUV background.

Table 45. Possible components of a diffuse extragalactic far ultraviolet background with their estimated intensities ${ }^{a}$

\begin{tabular}{lc}
\hline Summed from all galaxies & 40 to 200 \\
QSOs/AGNs & $<10$ \\
Intergalactic medium & $<10$ \\
\hline observed upper limit & 50 to 300 \\
\hline
\end{tabular}

${ }^{a}$ Intensities are given in units of photons $\mathrm{cm}^{-2} \mathrm{~s}^{-1} \mathrm{sr}^{-1} \AA^{-1}$.

\subsection{Visual}

A selection of upper limits from photometric measurements as well as lower limits obtained from galaxy counts are summarised in Table 46. In the table, the author(s) and date of publication are given in Col. (1). The wavelength of observation and the $I_{\mathrm{EBL}}$ value (or its upper limit) as given in the original publication are listed in Cols. (2) and (3). In Col. (4) we give our critical revision (upper limit) of each $I_{\mathrm{EBL}}$ value; in deriving these "revised values" we have tried to consider the effects of some additional uncertainties or corrections which in our opinion were not sufficiently discussed in the original paper. In Col. (5) we give $\lambda I_{\lambda}=\nu I_{\nu}$ for the revised EBL values. The last Col. (6) gives the method used. 
Table 46. Observational upper and lower limits to the EBL intensity as determined from surface photometry or galaxy counts

\begin{tabular}{|c|c|c|c|c|c|}
\hline Author(s) & $\begin{array}{c}\lambda \\
(\AA)\end{array}$ & $I_{\mathrm{EBL}}$ & $\begin{array}{c}I_{\mathrm{EBL}} \\
\text { revised, } 1 \sigma\end{array}$ & $\begin{array}{c}\lambda I_{\lambda} \text { (revised) } \\
\text { limits erg s }{ }^{-1} \mathrm{~cm}^{-2} \mathrm{sr}^{-1}\end{array}$ & Method \\
\hline$\overline{\text { Dube et al. (1979) }}$ & 5115 & $\begin{array}{l}1.0 \pm 1.2 S_{10} \\
\quad \leqq 3.4 S_{10}\end{array}$ & $\begin{array}{l}4.0 \pm 1.2 S_{10} \\
\leqq 5.2 S_{10}\end{array}$ & $\leqq 4.010^{-5}$ & photometry \\
\hline Toller (1983) & 4400 & $\begin{array}{l}1.3 \pm 1.3 S 10_{\odot} \\
\leqq 3.9 S 10_{\odot}\end{array}$ & $\begin{array}{l}1.9 \pm 4.8 S 10_{\odot} \\
\leq 6.7 S 10_{\odot}\end{array}$ & $\leqq 3.210^{-5}$ & photometry \\
\hline $\begin{array}{l}\text { Mattila \& Schnur } \\
(1990)\end{array}$ & 4000 & $6 . \overline{\overline{5}} \pm 2.5 \mathrm{cgs}^{*}$ & $\leqq 9.0 \mathrm{cgs}^{*}$ & $\leqq 3.610^{-5}$ & photometry \\
\hline Cowie et al. (1994) & $\begin{array}{c}3400\left(\mathrm{U}^{\prime}\right) \\
4470(\mathrm{~B}) \\
5425(\mathrm{~V}) \\
8340(\mathrm{I}) \\
22000(\mathrm{~K})\end{array}$ & & & $\begin{array}{ll}1.3 & 10^{-6} \\
1.8 & 10^{-6} \\
3.1 & 10^{-6} \\
4.7 & 10^{-6} \\
5.2 & 10^{-6}\end{array}$ & $\begin{array}{l}\text { galaxy counts } \\
\quad\left(K \leqq 22^{\mathrm{m}}\right)\end{array}$ \\
\hline Tyson (1995) & $\begin{array}{l}3600(\mathrm{U}) \\
4500(\mathrm{~B}) \\
6500(\mathrm{R}) \\
9000(\mathrm{I}) \\
22000(\mathrm{~K})\end{array}$ & & & $\begin{array}{c}2.5(+.07-.04) 10^{-6} \\
2.9(+.09-.05) 10^{-6} \\
2.9(+.09-.05) 10^{-6} \\
2.6(+.3-.2) 10^{-6} \\
7.2(+1-1) 10^{-6}\end{array}$ & $\begin{array}{l}\text { galaxy counts } \\
\left(B_{j} \leqq 29^{\mathrm{m}} / \square^{\prime \prime}\right)\end{array}$ \\
\hline $\begin{array}{l}\text { Morgan \& Driver } \\
\text { (1995) }\end{array}$ & $\begin{array}{l}4500(\mathrm{~B}) \\
5500(\mathrm{~V}) \\
6500(\mathrm{R}) \\
9000(\mathrm{I})\end{array}$ & & & $\begin{array}{ll}1.9 & 10^{-6} \\
1.3 & 10^{-6} \\
3.2 & 10^{-6} \\
3.5 & 10^{-6}\end{array}$ & $\begin{array}{l}\text { galaxy counts } \\
\quad\left(B \leqq 26^{\mathrm{m}}\right)\end{array}$ \\
\hline & $\begin{array}{l}4500(\mathrm{~B}) \\
5500(\mathrm{~V}) \\
6500(\mathrm{R}) \\
9000(\mathrm{I})\end{array}$ & & & $\begin{array}{c}4.710^{-6} \\
6.410^{-6} \\
8.210^{-6} \\
10.010^{-6}\end{array}$ & $\begin{array}{l}\text { galaxy counts } \\
\left(m_{\text {Filter }} \leqq 38^{\mathrm{m}}\right)\end{array}$ \\
\hline
\end{tabular}

$* \operatorname{cgs}=10^{-9} \operatorname{ergs~cm}^{-2} \mathrm{~s}^{-1} \operatorname{sterad}^{-1} \AA^{-1}$.

\subsubsection{Photometric upper limits}

Three surface photometric measurements are included in Table 46:

(1) Dube et al. (1979) observed the total night sky brightness from the ground in eleven high-latitude fields. As a mean value of the 11 fields Dube et al. gave an EBL+DGL value of $1.0 \pm 1.2 S_{10}$. Because it was not possible to estimate the DGL contribution the result was interpreted as a $2 \sigma$ upper limit to the EBL of $3.4 S_{10}$ or $5.110^{-9} \mathrm{ergs} \mathrm{cm}^{-2} \mathrm{~s}^{-1}$ sterad $^{-1} \AA^{-1}$. A basic problem with this method is that it starts with the total night-sky brightness which is a factor of $\approx 100$ brighter than the EBL. Thus, very accurate measurements of the absolute intensities of ZL and airglow are required. The most critical point in the data analysis of Dube et al. was the way they corrected for the airglow. They assumed that airglow is a linear function of sec $z$ and used linear extrapolation to $\sec z=0$ to eliminate airglow. This method is doubtful since the $\sec z$ - dependence of the airglow is not strictly linear but follows the so-called van Rhijn's (1921) law. Mattila et al. (1991) have reanalysed the airglow problem using, as far as possible, the observational values given in Dube et al. (1979) and in Dube (1976). They have thus found that Dube et al. probably have overestimated the airglow intensity by $\approx 3 S_{10}$. Thus the residual value for $\mathrm{EBL}+$ DGL should be increased by this amount, resulting in $I_{\mathrm{EBL}+\mathrm{DGL}}=4.0 \pm 1.2 S_{10}$ or an $1 \sigma$ upper limit of $5.2 S_{10}$.

(2) Toller (1983) utilized measurements of a photometer aboard Pioneer 10 as it moved out of the zodiacal dust cloud $(R \geqq 3.3 \mathrm{AU})$. From these he subtracted integrated starlight and gave a value for the average brightness of the diffuse background light of $I_{\mathrm{DGL}+\mathrm{EBL}}=3.3 \pm 1.2 S 10_{\odot}$ He estimated $I_{\mathrm{DGL}}$ to be $2.0 \pm 0.4 S 10_{\odot}$. As a final result Toller thus obtained an EBL intensity of $1.3 \pm 1.3$ $S 10_{\odot}$ which he expressed as a $2 \sigma$ upper limit of $I_{\mathrm{EBL}} \leqq$ $3.9 S 10_{\odot}$.

Since Toller's EBL value has been frequently cited as the EBL reference value, it deserves a detailed discussion of errors. The basic problem for his EBL determination is the large field of view $(2.3 \times 2.3 \mathrm{deg})$ of the photometer. Thus, the starlight entered with full weight into the measured sky brightness, and in order to derive the small residual EBL one must know the ISL very accurately in the Pioneer 10 photometric system. This was not fully the case. The ISL values of Roach \& Megill (RM, 1961) and Sharov \& Lipaeva (SL, 1973) are based on the HarvardGroningen (Pickering et al. 1918, 1923, 1924; van Rhijn 1929) and Mount Wilson starcounts (Seares et al. 1930) 
the magnitudes of which were calibrated by using photographic techniques. Sharov \& Polyakova (1972) have shown that the Harvard-Groningen photographic magnitude scales are in need of positive corrections of as much as $0.4 \mathrm{mag}$ to $0.5 \mathrm{mag}$ in order to reduce stars of 7 to $16 \mathrm{mag}$ from $m_{\mathrm{pg}}$ to the $B$ system. In their ISL summation SL tried to take these photometric corrections into account and thus their ISL values should be given the preference over the RM values. Then an average $I_{\mathrm{DGL}+\mathrm{EBL}}$ of 3.9 $S 10_{\odot}$, instead of $3.3 S 10_{\odot}$, is obtained. There is a the remaining systematic error of the Sharov and Lipaeva ISL values due to the scale errors which is at least $15 \%$. With an average ISL value of $25 S 10_{\odot}$ this amounts to $3.8 S 10_{\odot}$. The systematic error of the Pioneer 10 photometry itself (e.g. due to calibration) has been given as $8 \%$ (Weinberg \& Schuerman 1981), which for $25 S 10_{\odot}$ corresponds to $2.0 S 10_{\odot}$. A further uncertainty of $1.6 S 10_{\odot}$ results from variations in the cutoff for bright stars. The total error resulting from quadratically adding the systematic and statistical errors then is $4.8 S 10_{\odot}$.

Thus we end up with a revised EBL value of $I_{\mathrm{EBL}}=$ $1.9 \pm 4.8 S 10_{\odot}$, which corresponds to $2.3 \pm 5.710^{-9} \mathrm{ergs}$ $\mathrm{cm}^{-2} \mathrm{~s}^{-1}$ sterad $^{-1} \AA^{-1}$ or to a one $\sigma$ upper limit of 8.0 $10^{-9} \operatorname{ergs~cm}^{-2} \mathrm{~s}^{-1}$ sterad $^{-1} \AA^{-1}$.

(3) Mattila \& Schnur (1990), on the basis of their observations in the dark cloud area L1642, have presented a preliminary estimate for the EBL of $6.5 \pm 2.510^{-9} \mathrm{ergs}$ $\mathrm{cm}^{-2} \mathrm{~s}^{-1}$ sterad $^{-1} \AA^{-1}$. In this method an opaque dark cloud is used as a zero point where the EBL is negligible or at least much smaller than in the transparent surroundings of the cloud. Starlight, zodiacal light and the atmospheric components are eliminated in the differential surface brightness measurements used in this method. The main problem is the elimination of the scattered light (DGL) which is present both in the opaque parts of the cloud as well as in its (semi)transparent surroundings. In view of the preliminary character of the above-mentioned value we prefer to interpret it as an upper limit, $I_{\mathrm{EBL}} \leqq$ $9.010^{-9} \mathrm{ergs} \mathrm{cm}^{-2} \mathrm{~s}^{-1}$ sterad $^{-1} \AA^{-1}$.

\subsubsection{Galaxy counts}

Deep galaxy counts by Cowie et al. (1994), Tyson (1995) and Morgan \& Driver (1995) have provided estimates for the contribution of galaxies to the EBL. These lower limits to the EBL are given in Table 46 for several wavelength bands between $3400 \AA$ and $2.2 \mu \mathrm{m}$.

(1) The EBL values of Cowie et al. are for a $K$-bandlimited sample with $K \leqq 22$ mag. The $K$-band counts have a slope $(\operatorname{dlog} N(m) / \mathrm{d} m)=0.26$ at $K=22 \mathrm{mag}$ which implies that the surface brightness contribution per magnitude interval is converging. Contrary to this the $B$ band counts have a divergent slope $(\operatorname{dlog} N(m) / \mathrm{d} m)=$ 0.45 (Gardner et al. 1993). Thus it is suggested that the total EBL at $U$ to visual wavelengths may be substantially higher than the values given in the Table.
(2) The EBL values of Tyson are for a sample to an isophotal limiting magnitude of $29 B_{j}$ magnitude $\operatorname{arcsec}^{-2}$. The limiting magnitudes of the counts are $\approx 27 \mathrm{mag}$ at $B_{j}, 26 \mathrm{mag}$ at $R$, and $24 \mathrm{mag}$ at $I$. Galaxies fainter than $20 \mathrm{mag}$ at $B_{j}$ contribute about $75 \%$ of the EBL at $4500 \AA$. Most of the EBL flux originates from galaxies around $B=24 \mathrm{mag}$.

(3) The EBL values of Morgan \& Driver (1995) are from counts with limiting magnitudes of $B=26 \mathrm{mag}$, $V=24.5 \mathrm{mag}, R=26 \mathrm{mag}$ and $I=22.5 \mathrm{mag}$. Morgan $\&$ Driver adopted two approaches in estimating the EBL: firstly they used direct observations of the number counts to sum up the EBL to the limiting magnitude; secondly they used a dwarf-dominated luminosity function to extrapolate the number counts down to a limiting magnitude of $m_{\text {Filter }}=38 \mathrm{mag}$. The EBL values for the second case are seen to be a factor of 2 to 5 higher than for the first case. This gives an impression of the possible importance of the contribution by very faint galaxies, $m \geqq 30 \mathrm{mag}$, to the EBL.

\subsection{Infrared}

The Diffuse Infrared Background Experiment (DIRBE) aboard the Cosmic Background Explorer (COBE) was designed to perform a sensitive search for the Cosmic Infrared Background Radiation (CIBR). Special care was paid in the design to supression of stray radiation. During the 10-month cryogenic operation of COBE in 1989 - 1990 DIRBE mapped the whole sky with high redundancy in ten wavelength bands between 1.25 and $240 \mu \mathrm{m}$. DIRBE is completely free from any residual atmospheric effects or contamination by rocket exhaust which have made many of the previous balloon or rocket borne experiment results problematic. The main obstacles for a determination of the CIBR are the remaining strong foreground components which contribute to the infrared sky brightness with varying importance over the whole wavelength region. As detailed in previous section, these are the zodiacal light, the light of unresolved stars and the thermal emission by interstellar dust (cirrus). Since there is no distinct spectral signature known in the CIBR, the separation of the foreground components has to be based on modelling of their different spatial or broad band spectral distributions. In the case of the zodiacal component also its temporal variation during a year can be utilized. This modelling process is still being worked on by the DIRBE team. The most recent, still preliminary results have been presented by Hauser (1996). They are reproduced in Table 47 for the ten DIRBE bands as well as for selected wavelengths based on FIRAS data. The first column of results gives the upper limits on the CIBR based on the darkest spots observed on the sky. Because no foreground components were subtracted, these values are stringent upper limits to any isotropic component of the infrared sky brightness. In the second column of 
Table 47. Upper limits and claims of tentative detections of the cosmic infrared background radiation

\begin{tabular}{|c|c|c|c|c|c|}
\hline $\begin{array}{r}\lambda \\
\mu \mathrm{m} \\
\end{array}$ & $\begin{array}{c}\nu I_{\nu} \\
\mathrm{nW} \mathrm{\textrm {m } ^ { - 2 }} \mathrm{sr}^{-1} \\
\end{array}$ & Reference & $\begin{array}{c}\nu I_{\nu} \\
\mathrm{nW} \mathrm{\textrm {m } ^ { - 2 }} \mathrm{sr}^{-1} \\
\end{array}$ & Reference & Ref. \\
\hline 1.25 & $\begin{array}{ll}393 & \pm 13\end{array}$ & DIRBE dark sky & $50-104$ & DIRBE residual & 1 \\
\hline 2.2 & $150 \pm 5$ & " & $15-26$ & " & 1 \\
\hline 3.5 & $63 \pm 3$ & " & $15-24$ & " & 1 \\
\hline 4.9 & 192 & '" & $9-22$ & " & 1 \\
\hline 12 & \pm 310 & $\prime \prime$ & $102-164$ & $\prime \prime$ & 1 \\
\hline 25 & \pm 330 & " & $136-210$ & " & 1 \\
\hline 60 & \pm 22 & " & $31-42$ & " & 1 \\
\hline 100 & \pm 10 & '" & $20-35$ & " & 1 \\
\hline 140 & $57 \pm 6$ & " & $12-63$ & " & 1 \\
\hline 240 & $22 \quad \pm 2$ & " & $8-33$ & " & 1 \\
\hline 111 & 108 & FIRAS dark sky & & & 1 \\
\hline 143 & 63 & ' & & & 1 \\
\hline 250 & 30 & $\prime \prime$ & & & 1 \\
\hline 500 & 6 & " & & & 1 \\
\hline $500-5000$ & & & $680 / \lambda(\mu \mathrm{m})$ & FIRAS residual & 2 \\
\hline $400-1000$ & & & $3.4(\lambda / 400 \mu \mathrm{m})^{-3}$ & FIRAS residual & 3 \\
\hline $10-40$ & & & $6 \mathrm{~h}(\lambda / \mu \mathrm{m})^{0.55}$ & $\gamma$-ray method & 4 \\
\hline
\end{tabular}

References: ${ }^{1}$ Hauser (1996), ${ }^{2}$ Mather et al. (1994), ${ }^{3}$ Puget et al. (1996), ${ }^{4}$ Dwek \& Slavin (1994). $h=H_{0} / 100 \mathrm{~km} \mathrm{~s}^{-1} \mathrm{Mpc}^{-1}$.

For conversion of the units to MJy/sr see Table 6 .

results the range of DIRBE sky brightness residuals at high galactic latitude after subtraction of "best models" currently available for zodiacal light and emission, starlight and interstellar cirrus are given. These numbers, as emphasized by Hauser (1996), are still preliminary. The uncompleteness of the foreground modelling is reflected in the fact that the spectrum of the residual brightness at mid-IR wavelengths shows a resemblance with the zodiacal light spectrum. For ease of comparison, part of Table 6 on total infrared sky brightness in dark spots is repeated here in the left part of the table.

Kashlinsky et al. (1996) have tried to convert the smoothness of the spatial distribution of DIRBE light into upper limits to a CIBR radiation component coming from clustered matter evolving according to standard scenarios. They find that the upper limits to such a component between $1.25 \mu \mathrm{m}-100 \mu \mathrm{m}$ are by a factor of 4 to 100 lower than the Hauser et al. (1996) residual isotropic brightnesses given in Table 47. These values have to be taken with caution, however, since their derivation is strongly model-dependent.

Using COBE/FIRAS data Mather et al. (1994) have estimated that the CMB spectrum between $0.5 \mathrm{~mm}$ and $5 \mathrm{~mm}$ deviates from a $2.726 \mathrm{~K}$ blackbody shape by less than $0.03 \%$ of the peak intensity. Taking twice this amount as an upper limit to an additional CIBR implies $\nu B_{\nu} \leq$ $6.810^{-11} / \lambda(\mu \mathrm{m}) \mathrm{W} \mathrm{m}^{-2} \mathrm{sr}^{-1}$.

Puget et al. (1996) have claimed a tentative detection of far-IR CIBR using COBE/FIRAS data. They have modelled and removed the foreground components above $140 \mu \mathrm{m}$. For estimating the interstellar cirrus emission they used its correlation with HI 21-cm data, and for zodiacal emission its spectral and spatial distribution as determined at shorter wavelengths, $\lambda \leq 100 \mu \mathrm{m}$. The residual isotropic component claimed for the $400 \mu \mathrm{m}-1000 \mu \mathrm{m}$ range can be represented by $\nu B_{\nu} \approx 3.410^{-9}(\lambda / 400 \mu \mathrm{m})^{-3}$ $\mathrm{W} \mathrm{m}{ }^{-2} \mathrm{sr}^{-1}$.

An indirect method for measurement of the mid-IR CIBR is based on the spectra of $\gamma$-ray sources, since $\gamma$ rays interact with intergalactic IR-photons by pair production, giving rise to energy-dependent extinction. A recent application gives, for $\lambda \approx 10-40 \mu \mathrm{m}$, the result $\nu B_{\nu} \approx 6 h 10^{-9}(\lambda / \mu \mathrm{m})^{0.55} \mathrm{~W} \mathrm{~m}^{-2} \mathrm{sr}^{-1}$ (Dwek \& Slavin 1994). The result depends on the Hubble constant $h=H_{0} / 100 \mathrm{~km} \mathrm{~s}^{-1} \mathrm{Mpc}^{-1}$. This estimate is by a factor of $\approx 10$ lower that the DIRBE isotropic residuals at 10 and $25 \mu \mathrm{m}$, but fits nicely to the DIRBE isotropic residuals at shorter and longer wavelengths (see Fig. 79). Again, there are uncertainties in this method, since the intrinsic high energy gamma ray spectra before attenuation by interaction with the cosmic infrared radiation field are not really known.

\subsection{Overview on EBL observations}

Figure 79 summarises the current observational limits to the extragalactic background light in the wavelength range from $0.1 \mu \mathrm{m}$ to $1000 \mu \mathrm{m}$. In the visual and near-infrared 


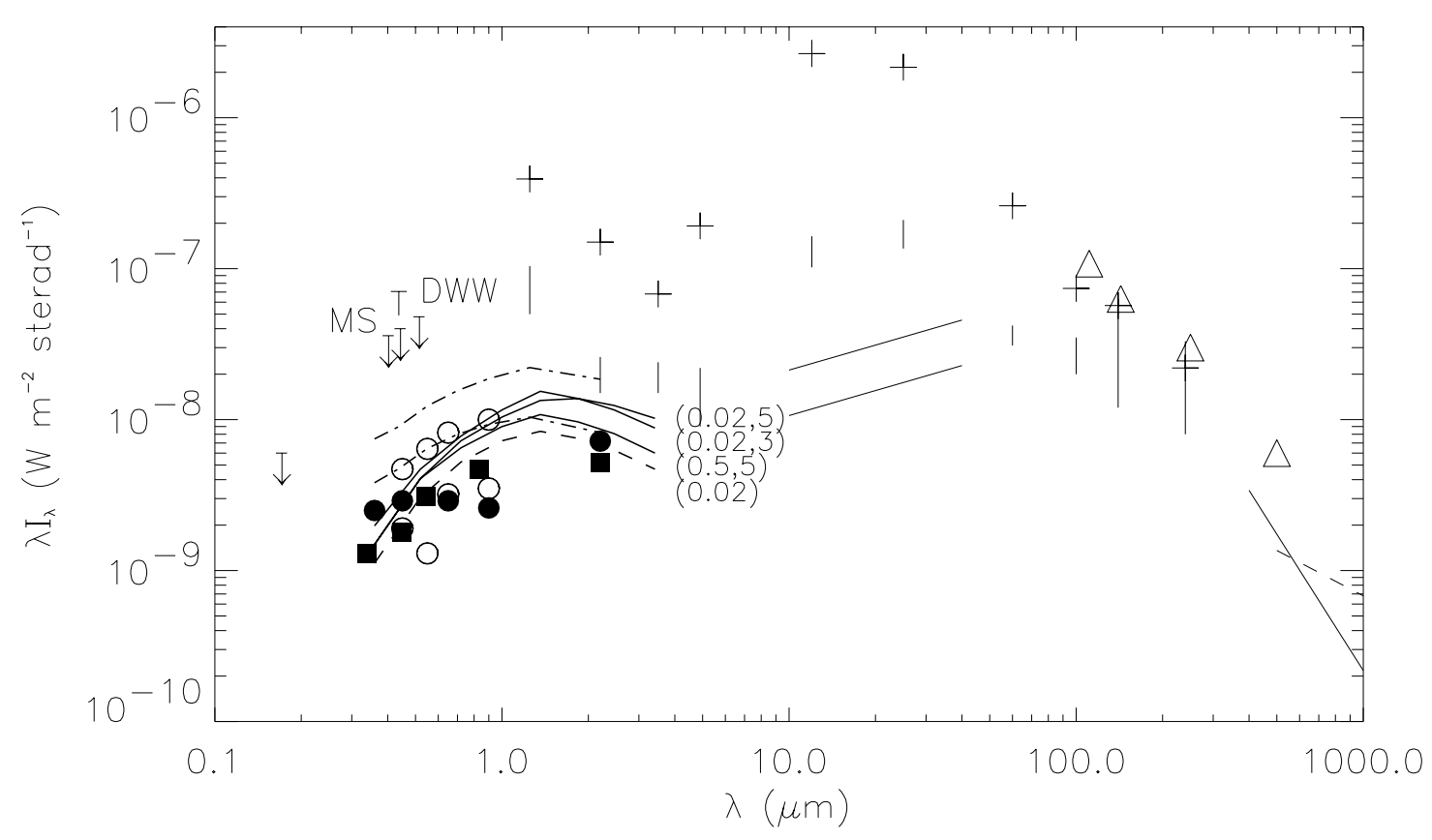

Fig. 79. Summary of present observational limits and model predictions for the EBL. The photometric upper limits by Dube et al. (DWW), Toller (T) and Mattila \& Schnur (MS) in optical and the UV upper limit of 300 photon units at $170 \mathrm{~nm}$ (see text) are shown as downward pointing arrows. The COBE/DIRBE and COBE/FIRAS dark sky (total) brightnesses between $1.25 \mu \mathrm{m}$ and $500 \mu \mathrm{m}$ are shown as crosses and open triangles, respectively. The ranges of isotropic residuals after foreground subtraction are shown by vertical bars for the DIRBE $1.25 \mu \mathrm{m}-240 \mu \mathrm{m}$ bands (Hauser 1996). The Mather et al. (1994) estimate for an upper limit of possible sub-mm excess above the CMB spectrum is shown as a dashed line between $500 \mu \mathrm{m}$ and $1000 \mu \mathrm{m}$. The claimed tentative detection of CIBR by Puget et al. (1996) is shown as a solid line between $400 \mu \mathrm{m}$ and $1000 \mu \mathrm{m}$. Solid lines at $10 \mu \mathrm{m}-40 \mu \mathrm{m}$ are the possible detections from Dwek \& Slavin (1994); the upper line is for $H_{0}=100 \mathrm{~km} \mathrm{~s}^{-1} \mathrm{Mpc}^{-1}$ and the lower one for $50 \mathrm{~km} \mathrm{~s}^{-1} \mathrm{Mpc}^{-1}$. The results from galaxy counts are are shown with different symbols: Cowie et al.: black squares; Tyson: solid circles; Morgan and Driver: open circles (two values at each wavelength band, see Table 46 and text). In the visual range, some model calculation results are shown as well for comparison: solid lines are after Yoshii \& Takahara (1988) for evolving galaxy models, labeled with $q_{0}$ and $z_{\mathrm{F}}$, where $z_{\mathrm{F}}$ means the epoch (measured by redshift) of galaxy formation; the dashed line is for a non-evolving galaxy model with $q_{0}=0.02$. The two dash-dotted lines are after Väisänen (1996) for models which include the estimated effect of low-surface-brightness and faint blue galaxy populations: the upper line is with Ferguson \& McGaugh (1995) luminosity function and with luminosity evolution (model labeled FMB-LE in Väisänen 1996); the lower line is with a luminosity function evolution in accordance with the findings of Lilly et al. (1995), i.e. extra brightening of the blue galaxies over the passive luminosity evolution, and an excess of a non-evolving blue population of faint galaxies (model labeled BBG in Väisänen 1996)

range, where discrepancies between different methods of determination are particularly large, we also plot a few selected model predictions for comparison, without the intent to discuss them here. Compared to the situation ten years ago, the gap between upper limits from direct measurements, lower limits from galaxy counts, and model predictions is getting smaller, being mostly less than a factor of ten by now. A comparison with the total sky brightness values shown in Fig. 1, which are typically brighter by two orders of magnitude, is informative. In this comparison please note that $\nu I_{\nu}$ and $\lambda I_{\lambda}$ are identical units of brightness.
Acknowledgements. We are grateful to M. Cohen for providing the estimates of integrated starlight shown in Figs. 61 and 62 and in Tables 24 and 29. We thank P. Feldman, R. MacQueen, H. Kimura and H. Lauche for helpful advice and discussions and M. Rowan-Robinson for supplying his infrared sky maps in digital form. We are grateful to P. Väisänen, who contributed the first and the last figure of this paper, and we very much thank D. Anders, K. Meissner-Dorn, and in particular M. Weckauf for the careful and patient work in producing or making computer-readable most of the numerous figures of this paper. 


\section{References}

Ábráham P., Leinert Ch., Lemke D., 1997, A\&A (in press)

Allen C.W., 1985, Astrophysical Quantities, third, reprinted edition. The Athlone Press, London, p. 162

Alonso A., Arribas S., Martínez-Roger C., 1995, A\&A 297, 197

Angel J.R.P, Woolf N.J., 1997, ApJ 475, 373

Arendt R.G., Berriman G.B., Boggess N., et al., 1994, ApJ 425, L85

Arendt R.G., et al., 1997 (in preparation)

Armand C., Milliard B., Deharveng J.M., 1994, A\&A 284, 12

Ashley M.C.B., Burton M.G., Storey J.W.V., Lloyd J.P., Bally J., Briggs J.W., Harper D.A., 1996, PASP 108, 721

Bahcall J.N., Soneira R.M., 1980, ApJS 44, 73

Bahcall J.N., Soneira R.M., 1984, ApJS 55, 67

Bandermann L.W., Wolstencroft R.D., 1976, Mem. R. Astron. Soc. 81 , pt. 2,37

Barbier D., 1956, The Airglow and the Aurorae, Special Suppl. No. 5 to the J. Atm. Terr. Phys., p. 38

Barth C.A., Schaffner S., 1970, JGR 75, 4299

Beckwith S.V.W., 1994, in "Star Formation and Techniques in Infrared and mm-Wave Astronomy", Ray T.R. and Beckwith S.V.W. (eds.), Lecture Notes in Physics 431. Springer-Verlag Berlin, Heidelberg, New York, p. 157

Becvar A., 1962, Atlas Coeli 1950.0, Atlas of the Heavens. Sky Publishing Company, Cambridge, Massachusetts

Beichman C.A., 1987, ARA\&A 25, 521

Bennett C.L., et al., 1994, ApJ 434, 587

Bernard J.-P., Boulanger F., Desert F.X., et al., 1994, A\&A 291, L5

Berriman G.B., Boggess N.W., Hauser M.G., Kelsall T., Lisse C.M., Moseley S.H., Reach W.T., Silverberg R.F., 1994, ApJL 431, L63

Berry R.L., 1976, J.R.A.S. Canada 70, 97

Bertiau F.C., de Greve E., Treanor P.J., 1973, Publ. Vatican Obs. 1, 159

Bessell M.S., 1979, PASP 91, 589

Bessell M.S., Brett J.M., 1988, PASP 100, 1134

Blaauw A., Elvius T., 1965, in Galactic Structure, Stars and Stellar Systems V, Blaauw A. and Schmidt M. (eds.). Chicago: University of Chicago Press, p. 589

Blackwell D.E., Dewhirst D.W., Ingham M.F., 1967, Adv. Astron. Astrophys. 5, 1

Boggess N.W., et al., 1992, ApJ 397, 420

Bohlin R.C., Savage B.D., Drake J.F., 1978, ApJ 224, 132

Boksenberg A., et al., 1973, MNRAS 163, 291

Boulanger F., Perault M., 1988, ApJ 330, 964

Boulanger F., Abergel A., Bernard J.-P., et al., 1996, A\&A 312,256

Bowyer S., Leinert Ch., 1991, The galactic and extragalactic background radiation, IAU SYmposium No. 139. Kluwer, Dordrecht

Bowyer S., Sasseen T., Lampton M., Wu X., 1993, ApJ 415

Bowyer S., 1991, ARA\&A 29, 59

Broadfoot A.L., Kendall K.R., 1968, JGR 73, 426

Broadfoot A.L., Kumar S., 1978, ApJ 222, 1054

Brosch N., 1991, MNRAS 250, 780

Brückner G., Howard R.A., Koomen M.J., et al., 1995, Phys. 162,357

Campins H., Rieke G.H., Lebofsky M.J., 1985, AJ 90, 896

Caplan J., Grec G., 1979, A\&A 78, 335
Caulet A., Hook R.N., Fosbury R.A.E., 1994, A\&AS 108, 271

Cebula R.P., Feldman P.D., 1982, ApJ 263, 987

Cebula R.P., Feldman P.D., 1984, JGR 89, 9080

Chamberlain J.W., 1961, Physics of the Aurora and the Airglow. Academic Press, New York

Classen C., 1976, Ph.D. Diss., University of Bonn

COBE Diffuse Infrared Background Experiment (DIRBE) Explanatory Supplement, Version 1.2, Hauser M.G., Kelsall T., Leisawitz D. and Weiland J. (eds.), COBE Ref. Pub. No. 97-A (Greenbelt, MD: NASA/GSFC), available in electronic form from the NSSDC

Cohen M., 1993, AJ 105, 1860

Cohen M., 1994, AJ 107, 582

Cohen M., Sasseen T.P., Bowyer S., 1994, ApJ 427, 848

Cohen M., 1995, ApJ 444, 874

Cowie L.L., et al., 1994, ApJ 434, 114

Dachs J., 1970, A\&A 6, 155

Dave J.V., 1964, J. Opt. Soc. America 54, 307

Davies J.K., Sykes M.V., Reach W.T., et al., 1997, Icarus 127, 251

de Bary E., 1964, Appl. Opt. 3, 1293

de Bary E., Bullrich K., 1964, J. Opt. Soc. America 54, 1413

Dermott S.F., Nicholson P.D., Burns J.A., Houck J.R., 1984, Nat 312, 505

Dermott S.F., Nicholson P.D., Kim Y., Wolven B., Tedesco E.F., 1988, in "Comets to Cosmology", Lawrence A. (ed.), Lecture Notes in Physics 297. Springer-Verlag Berlin, Heidelberg, New York, p. 3

Dermott S.F., Jayaraman S., Xu Y.L., Gustafson B.Å.S., Liou J.C., 1994, Nat 369, 719

Dermott S.F., Jayaraman S., Xu Y.L., Grogan K., Gustafson B.A.S., 1996a, in "Unveiling the cosmic infrared background, Dwek E. (ed.), AIP Conf. Proc. 348. Woodbury, New York, p. 25

Dermott S.F., Grogan K., Gustafson B.Å.S., Jayaraman S., Kortenkamp S.J., Xu Y.L., 1996b, in "Physics, chemistry, and dynamics of interplanetary dust", Gustafson B.A.S. and Hanner M.S. (eds.), ASP Conf. Ser. 104. San Francisco, p. 143

Désert F.-X., Abergel A., Bernard J.-P., et al., 1996, Limits on the far infrared CIBR from DIRBE, FIRAS and HI surveys, in Dwek E. (ed.) Unveiling the cosmic infrared background, AIP Conf. Proc. 348, 96

Désert F.-X., Boulanger F., Puget J.-L., 1990, A\&A 237, 215

Deul E.R., Wolstencroft R.D., 1988, A\&A 196, 277

Draine B.T., Anderson N., 1985, ApJ 292, 494

Dring A., Murthy J., Henry R., Walker H., 1996, ApJ 457, 764

Dube R.R., 1976, Ph.D. Thesis, Princeton University

Dube R.R., Wickes W.C., Wilkinson D.T., 1979, ApJ 232, 333

Dumont R., 1965, A\&A 28, 265

Dumont R., Levasseur-Regourd A.-Ch., 1978, A\&A 64, 9

Dumont R., Levasseur-Regourd A.-Ch., 1985, Planet. Space Sci. 33,1

Dumont R., Sanchez Martinez F., 1966, A\&A 29, 113

Dumont R., Sanchéz F., 1976, A\&A 51, 393

Dürst J., 1982, A\&A 112, 241

Dwek E., Slavin J., 1994, ApJ 436, 696

Dwek E., et al., 1997, ApJ 475, 565

Elsässer H., Haug U., 1960, ZfA 50, 121

Elvey C.T., Roach F.E., 1937, ApJ 85, 213 
Epchtein N., et al., 1994, in "Science with astronomical nearinfrared sky surveys", Epchtein N., Omont A., Burton B., and Persi P., (eds.). Kulwer, Dordrecht, p. 3

Epchtein N., et al., 1997, ESO Messenger No. 87, March 1997, p. 27

Feldman P.D., 1977, A\&A 61, 635

Feldman P.D., eight co-authors 1992, Geophys. Res. Lett. 19, 453

Ferguson H.C., McGaugh S.S., 1995, ApJ 440, 470

Fixsen D.J., et al., 1994, ApJ 420, 457

Franceschini A., Mazzei P., De Zotti G., 1991a, In: RoccaVolmerange B., Deharveng J.M., Tran Thanh Van J. (eds.) The Early Observable Universe from Diffuse Backgrounds. Editions Frontieres, p. 249

Franceschini A., Toffolatti L., Mazzei P., Danese L., De Zotti G., 1991b, A\&AS 89, 285

Frank L.A., Craven J.D., Rairden R.L., 1985, Adv. Space Res. 5,53

Frey A., Hofmann W., Lemke D., Thum C., 1974, A\&A 36, 447

Frey A., Hofmann W., Lemke D., 1977, A\&A 54, 853

Furton D.G., Witt A.N., 1990, ApJ 364, L45

Furton D.G., Witt A.N., 1992, ApJ 386, 587

Gardner J.P., Cowie L.L., Wainscoat R.J., 1993, ApJ, L9

Garstang R.H., 1986, PASP 98, 364

Garstang R.H., 1988, Observatory 108, 159

Garstang R.H., 1989a, ARA\&A 27, 19

Garstang R.H., 1989b, PASP 101, 306

Garstang R.H., 1991, PASP 103, 1109

Giard M., Pajot F., Lamarre J.M., et al., 1988, A\&A 201, L1

Giard M., Pajot F., Lamarre J.M., Serra G., Caux E., 1989, A\&A 215, 92

Giese R.H., 1979, A\&A 77, 223

Giese R.H., Kneissel B., Rittich U., 1986, Icarus 68, 395

Gillett F.C., Stein W.A., 1971, ApJ 164, 77

Gillett F.C., Forrest W.J., Merrill K.M., 1973, ApJ 183, 87

Gondhalekar P.M., 1990, in: Bowyer S. and Leinert Ch. (eds.) Galactic and Extragalactic Background Radiation, Proc. of IAU Symposium No. 139. Kluwer, Dordrecht, p. 49

Gordon K.D., 1997, PhD Thesis, The University of Toledo

Gordon K.D., Witt A.N., Friedmann B.C., 1997, ApJ (submitted)

Gordon K.D., Witt A.N., 1997, ApJL (submitted)

Guhathakurta P., Tyson J.A., 1989, ApJ 346, 773

Haikala L.K., Mattila K., Bowyer S., Sasseen P., Lampton M., 1995, ApJ 443, L33

Hanner M.S., Weinberg J.L., De Shields II L.M., Green B.A., Toller G.N., 1974, J. Geophys. Res. 79, 3671

Hanner M.S., Sparrow J.G., Weinberg J.L., Beeson D.E., 1976, in: "Interplanetary Dust and Zodiacal Light", Elsässer H. and Fechtig H. (eds.), Lecture Notes in Physics 48. SpringerVerlag Berlin, Heidelberg, New York, p. 29

Hanner M., Leinert Ch., Pitz E., 1978, A\&A 65, 245

Hanner M.S., 1991, in "Origin and evolution of interplanetary dust", Levasseur-Regourd A.C. and Hasegawa H. (eds.), IAU Colloquium 126. Kluwer, Dordrecht, p. 171

Harrison A.W., Kendall D.J.W., 1973, Planet. Space Sci. 21, 1731

Hauser M.G., et al., 1984, ApJ 278, L15
Hauser M.G., 1988, in "Comets to cosmology", Lawrence A. (ed.), Lecture Notes in Physics 297. Springer-Verlag Berlin, Heidelberg, New York, p. 27

Hauser M.G., 1995b, in "Examining the Big Bang and Diffuse Background Radiations", IAU symposium No. 168, Kafatos M., Kondo Y., and Bowyer S. (eds.). Kluwer Academic Publishers, Dordrecht, p. 99

Hauser M.G., 1995a, in Cazetti D., Livio M. and Madau P. (eds.) Extragalactic Background Radiation. Cambridge University Press, p. 135

Hauser M.G., 1996, in "Unveiling the cosmic infrared background", Dwek E. (ed.), AIP Conf. Proc. 348. Woodbury, New York, p. 11

Hayes D.S., 1985, in "Calibration of fundamental stellar quantities", Hayes D.S., Pasinetti L.E. and Philip A.G.D. (eds.). Reidel Publishing Company, Dordrecht, p. 225

Henry R.C., Murthy J., 1995, in Cazetti D., Livio M. and Madau P. (eds.) Extragalactic Background Radiation. Cambridge University Press, p. 51

Henry R., 1991, ARA\&A 29, 89

Hodapp K.-W., MacQueen R.M., Hall D.N.B., 1992, Nat 355, 707

Hoffmann B., Tappert C., Schlosser W., et al., 1997, A\&A (in press)

Hofmann W., Lemke D., Thum C., Fahrbach U., 1973, Nat 243,140

Hofmann W., Frey A., Lemke D., 1974, Nat 250, 636

Hofmann W., Lemke D., Thum C., 1977, Appl. Opt. 16, 3125

Holberg J., 1986, ApJ 311, 969

Hovenier J.W., Bosma P.B., 1991, in: "Origin and Evolution of Interplanetary Dust", Levasseur-Regourd A.C. and Hasegawa H. (eds.). Kluwer Academic Publishers, Dordrecht, p. 155

Hurwitz M., Bowyer S., Martin C., 1991, ApJ 372, 167

Isobe S., Tanabe H., Hirayama T., Koma Y., Soegijo J., Baba N., 1985, in: Properties and interactions of interplanetary dust, Giese R.H. and Lamy P.L. (eds.). Reidel, Dordrecht, p. 49

Isobe S., Sateesh-Kumar A., 1993, Astrophys. Space Sci. 205, 297

Jakobsen P., 1991, in "The Early Observable Universe from Diffuse Backgrounds", Rocca-Volmerange B., Deharveng J.M., and Tran Thanh Van J. (eds.). Édition Frontières, p. 115

Jakobsen P., 1995, in Cazetti D., Livio M. and Madau P. (eds.) Extragalactic Background Radiation. Cambridge University Press, p. 75

Kaiser C.B., 1970, ApJ 159, 77

Kalinowski K.J., Roosen R.G., Brandt J.C., 1975, PASP 87, 869

Kashlinsky A., Mather J.C., Odenwald S., 1996, ApJ 473, L9

Kessler M., et al., 1996, A\&A 315, L27

Kimeswenger S., Hoffmann B., Schlosser W., Schmidt-Kaler T., 1993, A\&AS 95, 517

Koutchmy S., Lamy P.L., 1985, in: Properties and Interactions of Interplanetary Dust, Giese R.H. and Lamy P.L. (eds.). Reidel, Dordrecht, p. 63

Krisciunas K., 1990, PASP 102, 1052

Kuhn J.R., Lin H., Lamy P., Koutchmy S., Smartt R.N., 1994, in: Infrared Solar Physics, Rabin DM. et al. (eds.), IAU syposium No. 154. Kluwer, Dordrecht, p. 185 
Kwon S.M., Hong S.S., J.L. Weinberg, 1991, in "Origin and evolution of interplanetary dust", Levasseur-Regourd A.C. and Hasegawa H. (eds.), IAU Colloquium 126. Kluwer, Dordrecht, p. 179

Léger A., Mariotti J.-M., Mennesson B., et al., 1996, Icarus 123,249

Lehtinen K., Mattila K., 1996, A\&A 309, 570

Leinert Ch., 1975, Space Sci. Rev. 18, 281

Leinert C., 1990, in The Galactic and Extragalactic Background Radiation, Bowyer S. and Leinert C. (eds.). Dordrecht: Kluwer, p. 75

Leinert Ch., Link H., Pitz E., Giese R.H., 1976, A\&A 47, 221

Leinert Ch., Richter I., Pitz E., Hanner M., 1980, in: "Solid particles in the solar system", Halliday I. and McIntosh B.A. (eds.). D. Reidel Publishing Company, Dordrecht, p. 15

Leinert Ch., Hanner M., Richter I., Pitz E., 1980b, A\&A 82, 328

Leinert Ch., Pitz E., Link H., Salm N., 1981, Space Sci. Instrum. 5, 257

Leinert Ch., Richter I., 1981, A\&AS 46, 115

Leinert Ch., Richter I., Pitz E., Hanner M., 1982, A\&A 110, 355

Leinert Ch., Pitz E., 1989, A\&A 210, 399

Leinert Ch., 1990, in "The Galactic and Extragalactic Background Radiation", Bowyer S. and Leinert Ch. (eds.). Kluwer, Dordrecht, p. 75

Leinert Ch., Grün E., 1990, Interplanetary Dust, in: Physics and Chemistry in Space, Schwenn R. and Marsch E. (eds.) - Space and Solar Physics 20. Springer, Berlin, Heidelberg, p. 207

Leinert Ch., Väisanen P., Mattila K., Lehtinen K., 1995, A\&AS 112, 99

Lemke D., et al., 1997, A\&A (submitted)

Léna P., Viala Y., Hall D., Soufflot A., 1974, A\&A 37, 81

Levasseur A.-Ch., Blamont J.E., 1973, Nat 246, 26

Levasseur A.-Ch., Blamont J.E., 1975, Space Res. XV, 573

Levasseur A.-Ch., Meier R.R., Tinsley B.A., 1976, JGR 81, 5587

Levasseur-Regourd, A.-Ch., Dumont R., 1980, A\&A 84, 277

Levasseur-Regourd A.-Ch., 1996, in "Physics, chemistry, and dynamics of interplanetary dust", Gustafson Bo A. S. and Hanner M.S. (eds.), Astr. Soc. Pac. Conf. Ser. 104. San Francisco, p. 301

Lillie C.F., 1968, Ph.D. Thesis, University of Wisconsin

Lillie C.F., 1972, in "The scientific results from OAO-2", Code A.O. (ed.), NASA SP-310. Washington, p. 95

Lilly S.J., LeFevre O., Crampton D., Hammer F., Tresse L., 1995, ApJ 455, 50

Lockman F.J., Jahoda K., McCammon D., 1986, ApJ 302, 432

Longair M.S., 1995, In Sandage A.R., Kron R.G. and Longair M.S. (eds.) The Deep Universe. Springer, Berlin, Heidelberg, New York, p. 317

Lonsdale C.J., 1995, in Cazetti D., Livio M. and Madau P. (eds.) Extragalactic Background Radiation. Cambridge University Press, p. 145

Louistisserand S., Bücher A., Koutchmy S., Lamy Ph., 1987, A\&AS 68,539

Low F.J., et al., 1984, ApJL 278, L19

Lyutyi V.M., Sharov A.S., 1982, AZh 59, 174

MacQueen R.M., 1968, ApJ 154, 1059
MacQueen R.M., Greeley B.W., 1995, ApJ 154, 1059

MacQueen R.M., Hodapp K.-H., Hall D.N.B., 1994, Infrared Solar Physics, Rabin D.M. et al. (eds.), IAU symposium No. 154. Kluwer, Dordrecht, p. 199

MacQueen R.M., Ross C.L., Mattingly T., 1973, Planet. Space Sci. 21, 2173-2179

McCaughrean M.J., 1988, "The astronomical application of infrared array detectors", Ph.D. Thesis, University of Edinbourgh

McNally D., 1994, The Vanishing Universe, Adverse Environmental Impacts on Astronomy. Cambridge University Press

Maihara T., Mizutani K., Hiromoto N., Takami H., Hasegawa H., 1985, in: Properties and Interactions of Interplanetary Dust, Giese R.H. and Lamy P.L. (eds.). Reidel, Dordrecht, p. 63

Mankin W.G., MacQueen R.M., Lee R.H., 1974, A\&A 31, 17

Mann I., 1992, A\&A 261, 329

Mann I., 1993, Planet. Space Sci. 41, 301

Martin C., Bowyer S., 1990, ApJ 350, 242

Massey P., Gronwall, Pilachowski, 1990, PASP 102, 1046

Mather J.C., et al., 1994, ApJ 420, 439

Mathis J.S., Mezger P.G., Panagia N., 1983, A\&A 128, 212

Matsumoto T., 1990, in: Bowyer S. and Leinert Ch. (eds.) Galactic and Extragalactic Background Radiation, Proc. of IAU Symposium No. 139. Kluwer, Dordrecht, p. 317

Matsumoto S., Matsuura S., Noda M., 1994, PASP 106, 1217

Matsumoto T., Kawada M., Murakami H., Noda M., Matsuura S., Tanaka M., Narita K., 1996, PASJ 48, L47

Matsuura S., Matsumoto T., Matsuhara H., Noda M., 1995, Icarus 115,199

Mattila K., 1973, Sterne und Weltraum 12, 246

Mattila K., 1970, A\&A 8, 273

Mattila K., 1971, A\&A 15, 292

Mattila K., 1979, A\&AS 39, 53

Mattila K., 1980, A\&A 78, 253

Mattila K., 1990, in: Bowyer S. and Leinert Ch. (eds.) Galactic and Extragalactic Background Radiation, Proc. of IAU Symposium No. 139. Kluwer, Dordrecht, p. 257

Mattila K., Schnur G., 1990, see Mattila, 1990

Mattila K., Leinert Ch., Schnur G., 1991, in: RoccaVolmerange B., Deharveng J.M., Tran Thanh Van J. (eds.) The Early Observable Universe from Diffuse Backgrounds. Editions Frontieres, p. 133

Mattila K., Väisänen P., v. Appen-Schnur G.F.O., 1996a, A\&AS 119, 153

Mattila K., Lemke D., Haikala L.K., et al., 1996b, A\&A 315, L353

Maucherat-Joubert M., Cruvellier P., Deharveng J.M., 1979, A\&A 74, 218

Meier R.R., 1991, Space Sci. Rev. 58, 1

Meier R.R., Carruthers G.R., Page T.L., Levasseur-Regourd A.-Ch., 1977, JGR 82, 737

Michard M., Dollfus A., Pecker I.C., Laffineur M., d'Azambuja M., 1954, Ann. d'Astrophys. 17, 320

Misconi N.Y., 1977, A\&A 61, 497

Mizutani K., Maihara T., Hiromoto N., Takami H., 1984, Nat 312, 134-136

Morgan D.H., 1978, A\&A 70, 543 
Morgan I., Driver S.P., 1995, in Cazetti D., Livio M. and Madau P. (eds.) Extragalactic Background Radiation. Cambridge University Press, p. 285

Morgan D.H., Nandy K., Thompson G.I., 1976, M.N. 177, 531

Morrison D., Feldman P.D., Henry R.C., 1992, JGR 97, 1633

Mukai T., Yamamoto T., 1979, PASJ 31, 585

Murakami H., et al., 1994, ApJ 428, 354

Murakami H., et al., 1996, PASJ 48, L41

Murdock T.L., Price S.D., 1985, AJ 90, 375

Murthy J., Henry R.C., Feldman P.D., Tennyson P.D., 1990 A\&A 231, 187

Neckel H., Labs D., 1984, Solar Phys. 90, 205

Neugebauer G., et al., 1984, ApJ 278, L1

Neugebauer G., Wheelock S., Gillett F., et al., 1988, IRAS catalogs and atlases, Volume 1: Explanatory Supplement, Beichman C.A. et al. (eds.) NASA RP-1190. Washington, VI-21

Nguyen H.T., Rausche B.J., Severson S.A., et al., 1996, PASP 108,718

Nishimura T., 1973, PASJ 25, 375

Oliva E., Origlia L., 1992, A\&A 254, 466

Onaka T., Yamamura I., Tanabe T., Roellig T.L., Yuen L., 1996, PASJ 48, L59

Osterbrock D.E., Walker M.F., Koski T.A., 1976, PASP 88, 349

Osterbrock D.E., Martel A., 1992, PASP 104, 76

Page T., Carruthers G., Heckathorn H., 1982, NRL Report 8487

Paresce F., Jakobsen P., 1980, Nat 288, 119

Pennington R.L., Humphreys R.M., Odewahn S.C., Zumach W., Thurmes P.M., 1993, PASP 105, 521

Perrin J.-M., Darbon S., Sivan J.-P., 1995, A\&A 304, L21

Perrin J.-M., Sivan J.-P., 1992, A\&A 255, 271

Peterson A.W., 1963, ApJ 138, 1218

Peterson A.W., 1967, ApJ 148, L37

Peterson A.W., 1969, ApJ 155, 1009

Pfleiderer J., Mayer U., 1971, AJ 76, 692

Pickering E.C., Kapteyn J.C., vanRhijn P.J., 1918, Ann. Harvard College Obs. 101

Pickering E.C., Kapteyn J.C., vanRhijn P.J., 1923, Ann. Harvard College Obs. 102

Pickering E.C., Kapteyn J.C., vanRhijn P.J., 1924, Ann. Harvard College Obs. 103

Pilachowski C., Afriano J., Goodrich B., Binkert W., 1989, PASP 101, 707

Pitz E., Leinert Ch., Schulz A., Link H., 1979, A\&A 74, 15

Pröll J., 1980, Diploma Thesis, Ruhr-University Bochum

Puget J.-L., et al., 1996, A\&A 308, L5

Ramsay S.K., Mountain C.M., Geballe T.R., 1992, MNRAS 259,751

Raurden R., Frank L., Craven J., 1986, JGR 91, 13613

Reach W.T., 1988, ApJ 335, 468

Reach W.T., 1991, ApJ 369, 529

Reach W.T., 1992, ApJ 392, 289

Reach W.T., et al., 1995a, ApJ 451, 188

Reach W.T., Franz B.A., Weiland J.L., et al., 1995b, Nat 374, 521

Reach W.T., Franz B.A., Kelsall T., Weiland J.L., 1996a, in "Unveiling the cosmic infrared background", Dwek E. (ed.), AIP Conf. Proc. 348. Woodbury, p. 37

Reach W.T., et al., 1996b, A\&A 315, L381
Reed E.I., Blamont J.E., 1967, Space Res. VII, 337

Richter I., Leinert Ch., Planck B., 1982, A\&A 110, 115

Rieke G.H., Lebofsky M.J., Low F.J., 1985, AJ 90, 900

Ristorcelli I., Giard M., M'eny C., et al., 1994, A\&A 286, L23

Roach F.E., 1964, Space Sci. Rev. 3, 512

Roach F.E., Gordon, J.L., 1973, The Light of the Night Sky. D. Reidel Publ. Company, Dordrecht

Roach F.E., Meinel A.B., 1955, ApJ 122, 530

Roach F.E., Megill L.R., 1961, ApJ 133, 228

Rocca-Vomerange B., Deharveng J.M., Tran Thanh Van J., 1991, The early observable universe from diffuse backgrounds. Éditions Frontières, Gif-sur-Yvette

Röser S., Staude H.J., 1978, A\&A 67, 381

Rowan-Robinson M., Hughes J., Vedi K., Walker D.W., 1990, MN 246, 273

Rowan-Robinson M., Hughes J., Jones M., et al., 1991, MN 249,729

Scheffler H., 1982, Landolt-Börnstein VI/2c, 176

Schlosser W., 1972, Habilitationsschrift, Ruhr-University Bochum

Schmidt T., Leinert C., 1966, Z. f. Ap. 64, 110

Schmidtobreick L., 1997, Ph.D. Thesis, Universität Bochum

Schuerman D.W., Weinberg J.L., Beeson D.E., 1977, BAAS 9, 313

Schuerman D.W., Tanabe H., Weinberg J.L., Toller G.N., Beeson D.E., 1977, Abstract, COSPAR, Tel Aviv

Seares. F.H., Kapteyn J.C., van Rhijn P.J., 1930, Mount Wilson Catalogue of Photographic Magnitudes in Selected Areas 1-139, Carnegie Inst. Washington Publ. No. 402

Seidensticker K., Schmidt-Kaler T., Schlosser W., 1982, A\&A 114, 60

Sellgren K., 1984, ApJ 277, 623

Sellgren K., Werner M.W., Dinerstein H.L., 1983, ApJ 271, L13

Sharov A.S., Lipaeva N.A., 1973, Soviet Astr. 17, 69

Sharov A.S., Polyakova G.I., 1972, Soobshch. Gos. Astron. Inst. Shternberga, No. 177, 3

Silverberg R.F., et al., 1993, Proc. SPIE Conf. 2019, Infrared Spaceborne Remote Sensing, Scholl M.S. (ed.). Bellingham: SPIE, p. 180

Sivan J.-P., Perrin J.-M., 1993, ApJ 404, 258

Smith L.L., Roach F.E., Owen R.W., 1970, Batelle Institute Report BNWL-1419-UC-2

Sodroski T.J., Odegard N., Arendt R.G., Dwek E., Weiland J.L., Hauser M.G., Kelsall T., 1997, ApJ 480, 173

Sparrow J.G., Ney E.P., 1968, ApJ 154, 783

Sparrow J.G., Ney E.P., 1972, ApJ 174, 705

Sparrow J.G., Ney E.P., 1972, ApJ 174, 717

Sparrow J.G., Weinberg J.L., 1976, in: "Interplanetary Dust and Zodiacal Light", Elsässer H. and Fechtig H. (eds.), Lecture Notes in Physics 48. Springer-Verlag Berlin, Heidelberg, New York, p. 41

Spiesman W.J., et al., 1995, ApJ 442, 662

Stark A.A., Gammie C.F., Wilson R.W., Bally J., Linke R.A., Heiles C., Hurwitz M., 1992, ApJS 79, 77

Staude H.J., 1975, A\&A 39, 325

Sykes M.V., 1985, Icarus 85, 267

Sykes M.V., Lebofsky L.A., Hunten D.M., Low F., 1986, Sci. 232,1115

Sykes M.V., 1988, ApJL 334, L55

Sykes M.V., Lien D.J., Walker R.G., 1990, Icarus 86, 236 
Sykes M.V., Walker R.G., 1992, Icarus 95, 180

Tanabe H., 1973, in "Papers on the night sky and airglow continuum at Chichijima", World data center C2 (Airglow), Tokyo Astron. Obs. 45

Taylor B.J., 1992, PASP 104, 500

Tennyson P.D., Henry R.C., Feldman P.D., Hartig G.F., 1988, ApJ 330, 435

Thomas G.E., 1978, Ann. Rev. Earth Planet. Sci. 6, 173

Toller G.N., 1981, Ph.D. Thesis, State University of New York at Stony Brook

Toller G.N., 1983, ApJ 266, L79

Toller G.N., Tanabe H., Weinberg J.L., 1987, A\&A 188, 24

Toller G.N., 1990, in: Bowyer S. and Leinert Ch. (eds.) Galactic and Extragalactic Background Radiation, Proc. of IAU Symposium No. 139. Kluwer, Dordrecht, p. 21

Toller G.N., Weinberg J.L., 1985, in: "Properties and Interactions of Interplanetary Dust", Giese R.H. and Lamy Ph. (eds.). D. Reidel Publishing Company, Dordrecht, p. 21

Tollestrup E.V., Fazio G.G., Woolaway J., Blackwell J., Brecher K., 1994, in: Infrared Solar Physics, Rabin D.M. et al. (eds.), IAU 1994, The Netherlands, p. 179

Torr M.R., Torr D.G., Eun J.W., 1985, JGR 90, 4427

Treanor P.J., 1973, Observatory 93, 117

Tüg H., Schmidt-Kaler Th., 1982, A\&A 105, 400

Turnrose B.E., 1974, PASP 86, 512

Tyson J.A., 1990, in: Bowyer S. and Leinert Ch. (eds.) Galactic and Extragalactic Background Radiation, Proc. of IAU Symposium No. 139. Kluwer, Dordrecht, p. 245

Tyson J.A., 1995, in Cazetti D., Livio M. and Madau P. (eds.) Extragalactic Background Radiation. Cambridge University Press, p. 103

Väisänen P., 1996, A\&A 315, 21

Vande Noord E.L., 1970, ApJ 161, 309

van Dijk M.H.H., Bosma P.B., Hovenier J.W., 1988, A\&A 201, 373

van Rhijn P.J., 1921, Publ. Astr. Lab. Groningen 31, 1

van Rhijn P.J., 1929, Publ. Astr. Lab. Groningen No. 43

Vrtilek J., Hauser M.G.n 1995, ApJ 455, 677

Wainscoat R.J., Cowie L.L., 1992, AJ 103, 332
Wainscoat R.J., Cohen M., Volk K., Walker H. J., Schwartz D.E., 1992, ApJS 83, 111

Walker M.F., 1988, PASP 100, 496

Walker M.F., 1977, PASP 89, 405

Walker M.F., 1970, PASP 82, 674

Weiland J.L., Blitz L., Dwek E., Hauser M.G., Magnani L., Rickard L.J., 1986, ApJ 306, L101

Weinberg J.L., 1964, Ann. Astrophys. 27, 718

Weinberg J.L., Mann H.M., 1967, in "The zodiacal light and the interplanetary medium", Weinberg J.L. (ed.), NASA SP-150, Washington D.C., p. 1

Weinberg J.L., 1969, BAAS 1, 368

Weinberg J.L., Hanner M.S., Beeson D.E., De Shields L.M. III, Green B.A., 1974, JGR 79, 3664

Weinberg J.L., 1981, Sky and Telescope 61, 114

Weinberg J.L., Schuerman D.W., 1981, User's Guide for the Pioneer 10/11 background sky tape, NSSDC

Weinberg J.L., Hahn R.C., 1980, in: "Solid particles in the solar system", Halliday I. and McIntosh B.A. (eds.). D. Reidel Publishing Company, Dordrecht, p. 19

Wheelock S.L., et al., 1994, "IRAS Sky Survey Atlas Explanatory Supplement", JPL Publication 94-11 (Pasadena: JPL)

Wicenec A.J., 1995, Ph.D. Thesis, Universität Tübingen

Wicenec A.J., van Leeuwen F., 1995, A\&A 304, 160

Winkler C., Schmidt-Kaler T., Schlosser W., 1981, Mitt. Astr. Ges. 52, 163

Witt A.N., 1968, ApJ 152, 59

Witt A.N., Schild R.E., 1988, ApJ 325, 837

Witt A.N., Boroson T.A., 1990, ApJ 355, 182

Witt A.N., Lindell R.S., Block D.L., Evans Rh., 1994, ApJ 427, 227

Wright E.L., et al., 1991, ApJ 381, 200

Wolstencroft R.D., Brandt J.C., 1967, in "The zodiacal light and the interplanetary medium", Weinberg J.L. (ed.), NASA SP-150, Washington, p. 57

Yoshii Y., Takahara F., 1988, ApJ 326, 1

Zavarzin M.Yu., 1978, Astrophysics (Engl. Transl.) 14, 168 